A COMPARATIVE GENRE ANALYSIS OF BOUTIQUE HOTEL WEBPAGES IN THAILAND AND NEW ZEALAND

BY

\title{
PAKJIRA LEELERTPHAN
}

\author{
A thesis \\ submitted to the Victoria University of \\ Wellington in fulfilment of the \\ requirements for the degree of Doctor of \\ Philosophy
}

Victoria University of Wellington

2017 


\section{Dedication}

This thesis is dedicated to my wonderful mother, Ngarmjit Chaiwutti. Her support, encouragement, and love have sustained me throughout my life. 


\begin{abstract}
This study analyses the characteristics of boutique hotel websites in Thailand and New Zealand as a genre. The study has four main objectives: 1) to identify moves and steps of English boutique hotel websites in Thailand and New Zealand, 2) to examine visual images presented on boutique hotel websites in Thailand and New Zealand, 3) to compare and contrast the move structures and visual images of these websites, and 4) to explore contextual factors which influence the variation between move structures and the representation of visual images of these websites. Despite the increasing popularity of boutique hotels, there exists almost no research analysing these webpages as a genre. While studies have presented a general overview of the appearance and the popularity of the boutique hotel phenomenon, textual and visual analysis of boutique hotels remains under-investigated. Moreover, cross-cultural studies of hotel webpages are also limited.

Bhatia's (1993) and Kress and van Leeuwen's (2006) frameworks were adopted to investigate and analyse the genre of boutique hotel webpages. Bhatia's (1993) model was used to explore textual and ethnographic perspectives. Kress and van Leeuwen's (2006) visual analysis model was adopted to examine the ways in which visual images are depicted and organised in these hotel online advertisements. In addition to textual and visual analysis, interviews with hotel owners, managers, web designers, and web audiences were conducted. The purpose of these interviews was to explore the intentions and practices influencing the shape of the design and organisation of the hotel websites as a genre. Finally, using the frameworks of Bhatia (1993) and Kress and van Leeuwen (2006), the analysis of move structures and the visual design between the hotel webpages in the Thai and New Zealand datasets were compared.
\end{abstract}

This analysis shows minor variations across the two datasets in both textual and visual elements. The differences in terms of move structures between these two cultures are subtle, conforming to the same conventionality inherent within the respective genre, as it connects to their social context and functions. Consequently, my analysis opposes the notion that genre is cultural, proposed by Crossley (2007); Hyland (2000); and Mauranen (1993).

In regards to register analysis, the web designers in both cultures used similar distinctive lexico-grammatical features to persuade and engage the web audiences such as the personal pronoun you and we, imperatives, adjectives. Only the use of adjectives exhibits major differences, which reflects the uniqueness in a provision of services and facilities within these two countries. This study posits that the influential factors responsible for variation between these two datasets 
are a result of how the websites market their hotels and how the hotels operate. However, the interview data with the hotel owners and web designers did not clearly illustrate how they establish generic conventions and develop distinctive lexico-grammatical features. My participants suggested that they used their intuition or consulted other hotel webpages, in which they share a similar theme or concept when they developing their webpages.

In regards to the visual representation, the findings show that the depiction of the visual elements across the two countries generally does not differ. Rather, they seem to be reflect a homogeneous genre because they conform to the same practices. That is to say, the designs of the hotel webpages have their own integrity, in which the web designers usually follow. From a genre perspective, these similarities in terms of a representation of move structures and visual images between these two cultures are seen as either a recurring situation or reproduction of generic conventions of a particular genre, which is considered as a social action within a discourse community. 


\section{ACKNOWLEDGEMENTS}

I would like to express my gratitude to many people whose kind support and assistance made this study possible. First and foremost, I am greatly indebted to Dr. Jean Parkinson, my primary supervisor. Dr. Jean provided me valuable insights into genre-based research. She also has been patient, providing me with valuable comments and encouragement throughout my PhD journey. This thesis would not have been completed without her support.

I wish to express my sincere gratitude to Dr. Keith Comer, my secondary supervisor, who spent endless hours reading my numerous drafts with meticulous attention. His comments and attention to details helped me improve this thesis at every step.

I would also like to express my special thanks to the Student Learning Support, Victoria University of Wellington, particularly Emma Rowbotham, a learning advisor, who gave me important guidance about how to write and friendly help. Her logical way of explaining thesis structure has been of great value in organising my thesis.

My special appreciation is also extended to the School of Linguistics and Applied Language Studies for providing a supportive environment, and making my time here most enjoyable.

I would like to acknowledge my research participants for their time and insights even though they were busy.

I would also like to thank my PhD student colleagues, who have shared the PhD journey with me.

Most importantly, I would like to express my sincere gratitude to all my beloved family members for their love, understanding and encouragement. This thesis has been an outcome of their love and sacrifice. 


\section{Table of Content}

List of Tables

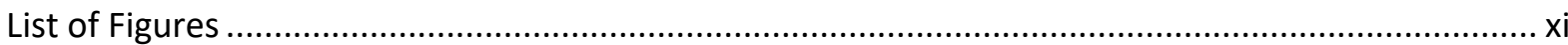

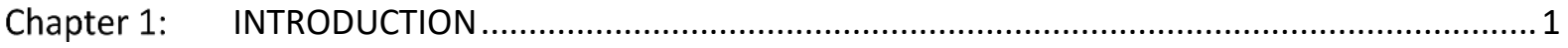

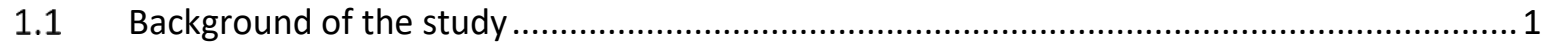

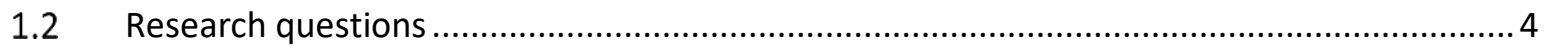

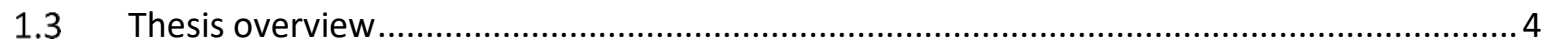

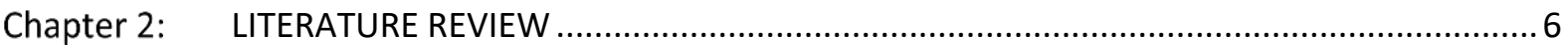

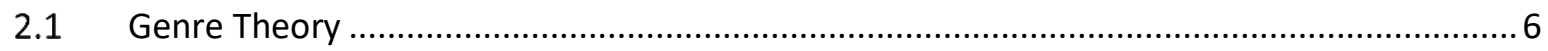

2.1.1 Importance of a Genre-Based approach ....................................................... 8

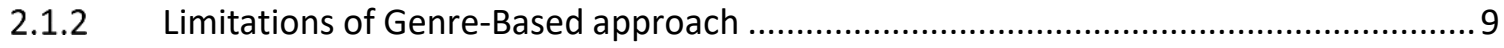

2.2 Hotel and tourism material advertising as a promotional genre..................................... 10

2.3 Tourism and hospitality research in boutique hotel context ....................................... 18

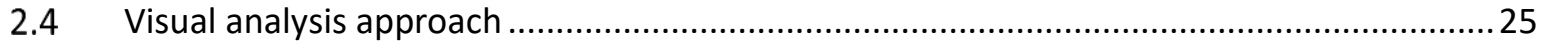

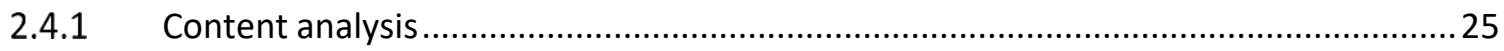

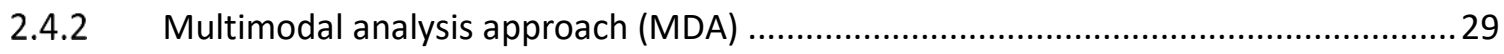

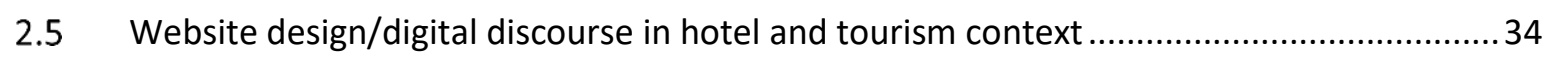

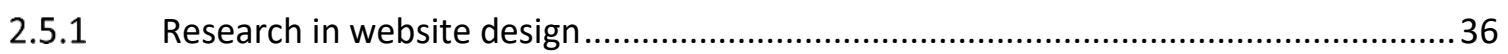

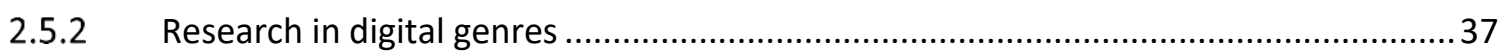

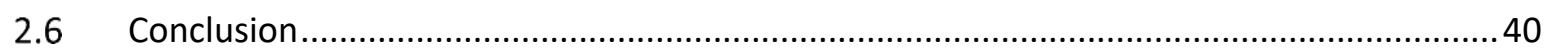

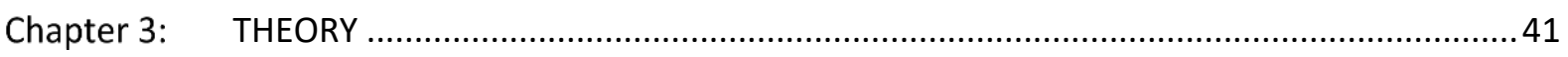

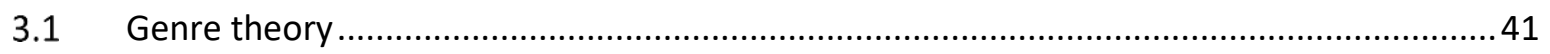

English for Specific Purposes (ESP) ................................................................... 41

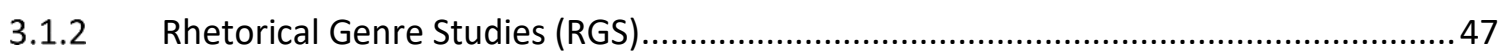

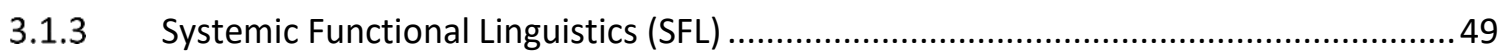

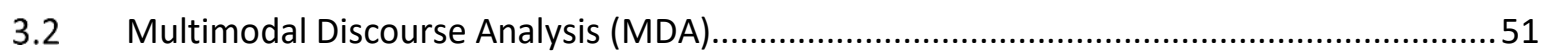

3.2.1 Multimodal approaches ......................................................................... 51

3.2.1.1 Social semiotic multimodality ................................................................. 52

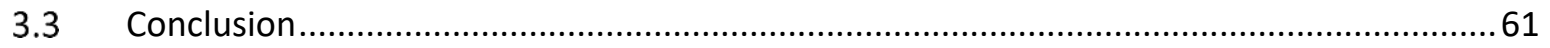

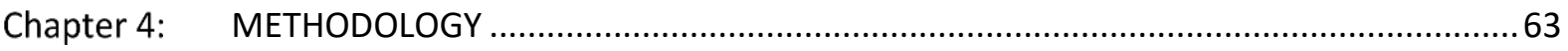

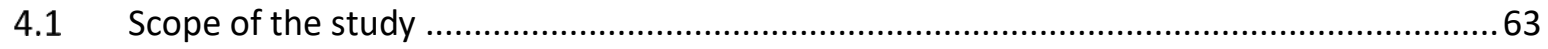

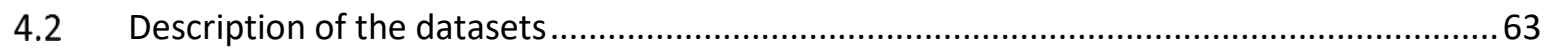

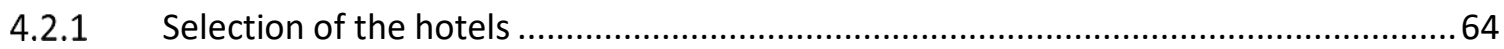

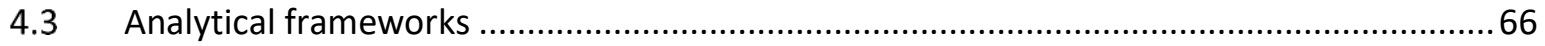




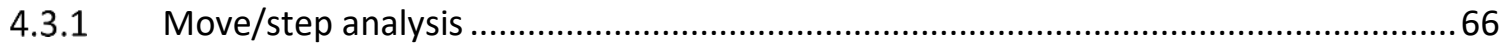

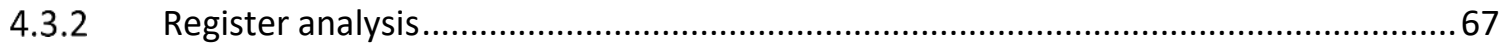

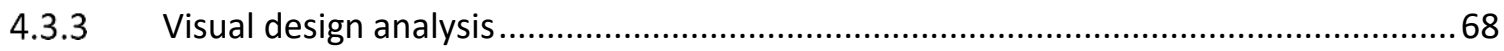

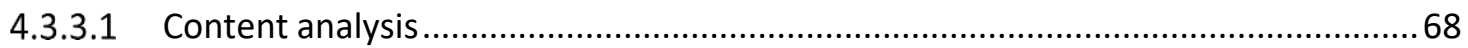

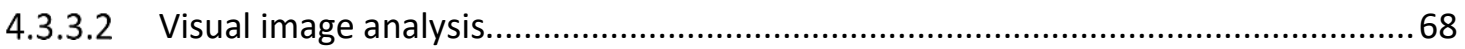

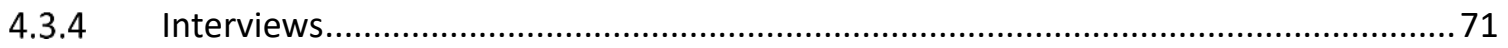

4.3.4.1 Interviews with hotel owners/web designers ....................................................... 72

4.3.4.2 Interviews with hotel website audience ................................................................. 75

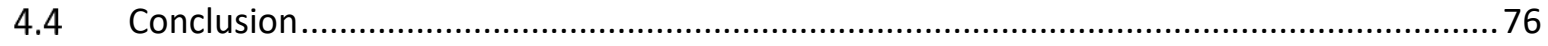

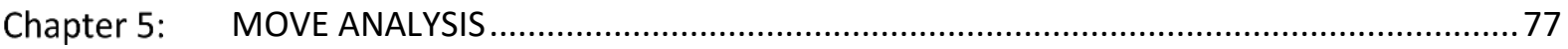

5.1 Identification of Moves and Steps in Thai and New Zealand boutique hotel webpages ..... 78

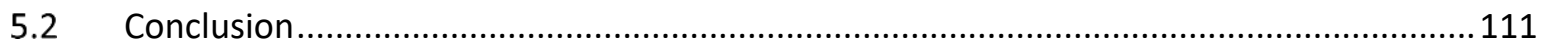

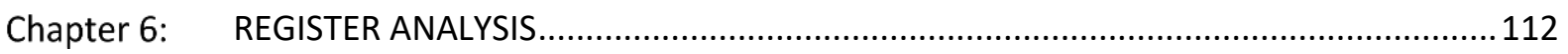

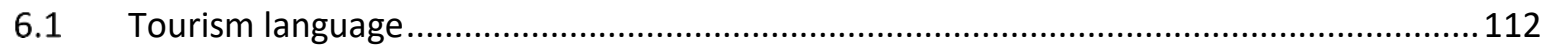

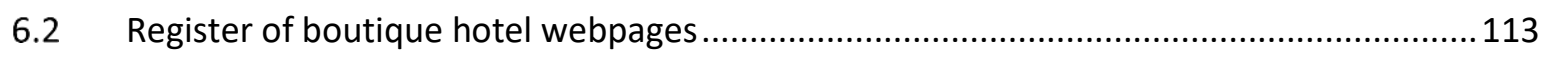

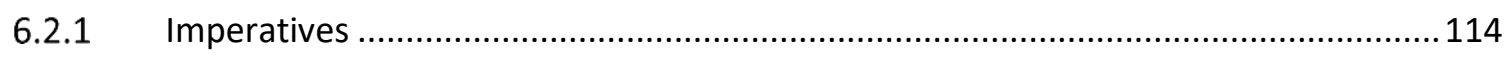

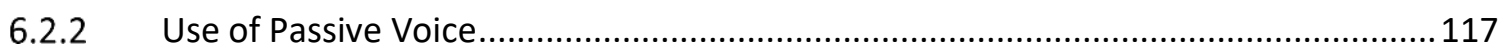

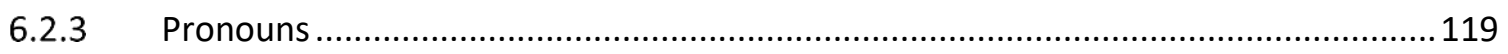

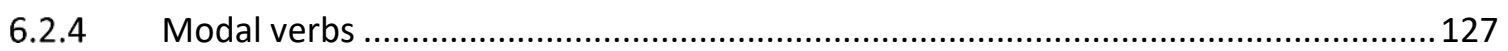

6.3 Register analysis of boutique hotel webpages: Comparative analysis ...............................131

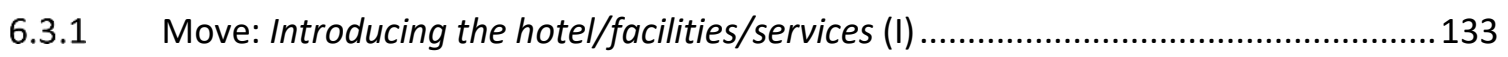

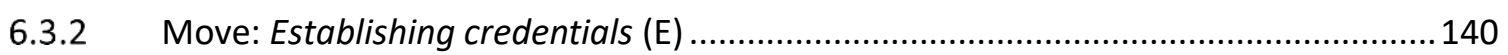

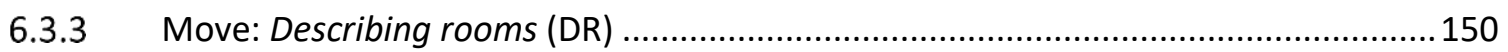

6.3.4 Move: Introducing restaurants/bars, lounges, and libraries (R)..............................159

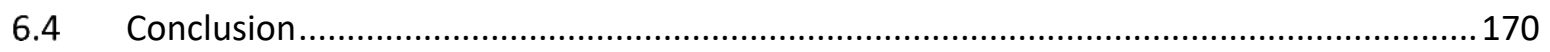

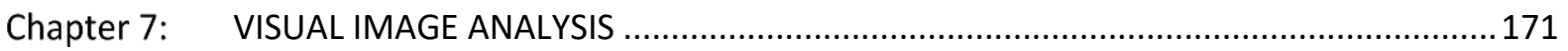

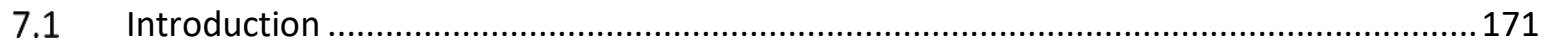

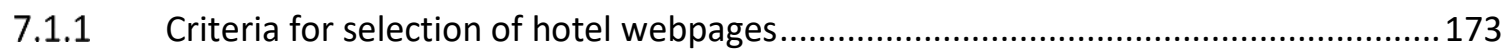

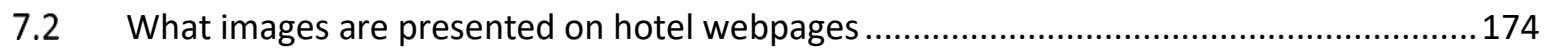

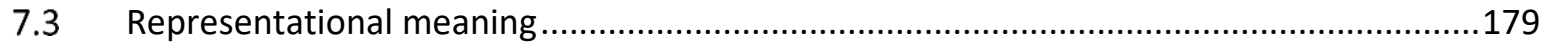

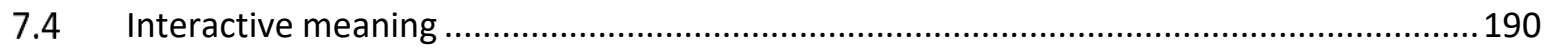

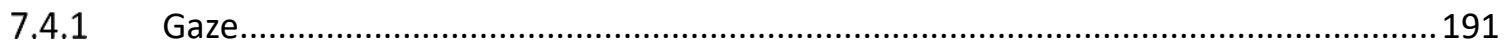

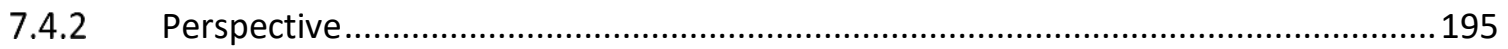

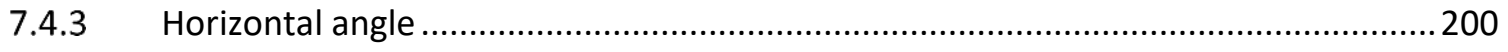

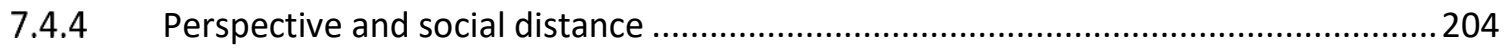

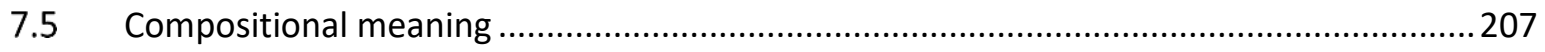




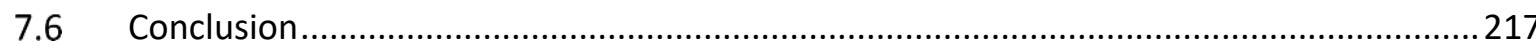

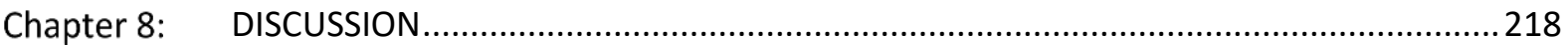

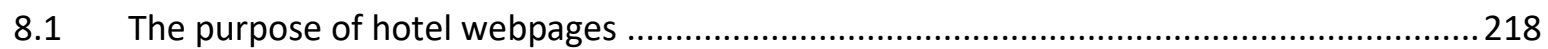

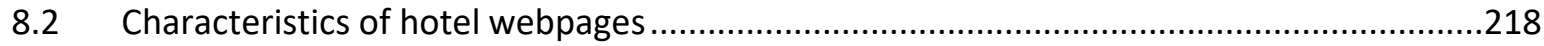

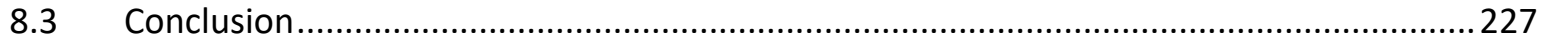

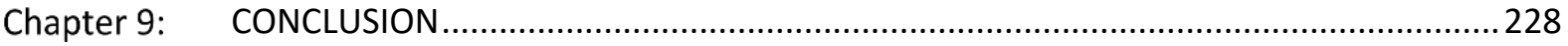

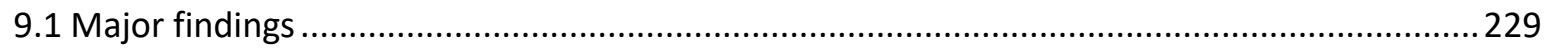

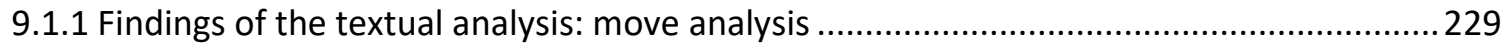

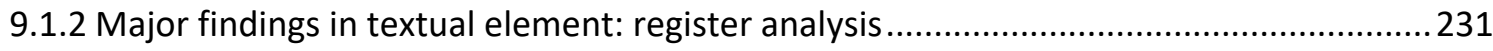

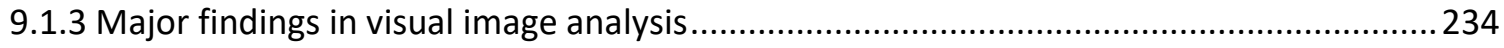

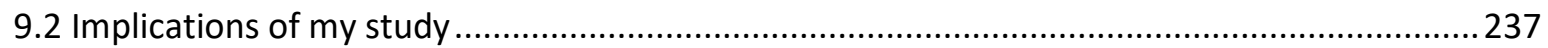

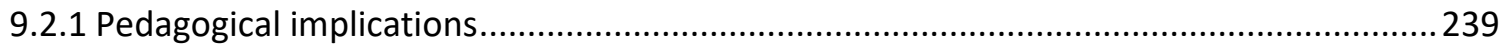

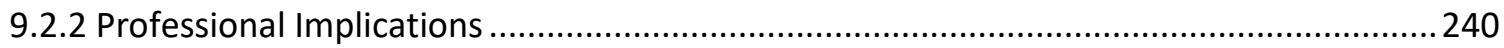

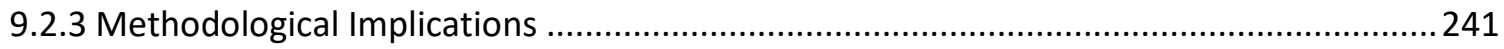

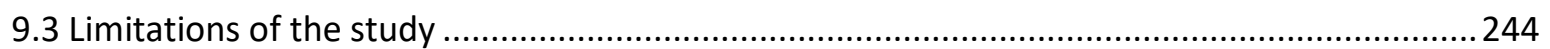

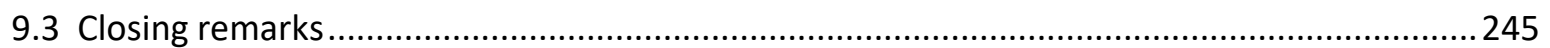

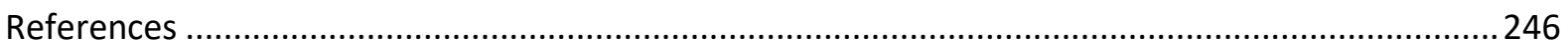

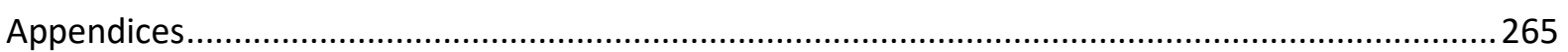

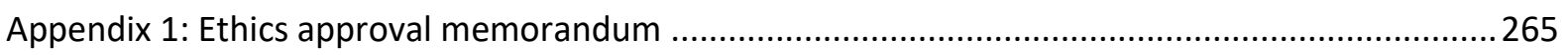

Appendix 2: Participant Consent Form for Hotels/Web Designers ...................................................266

Appendix 3: Information sheet for hotels's owners/web designers ...............................................267

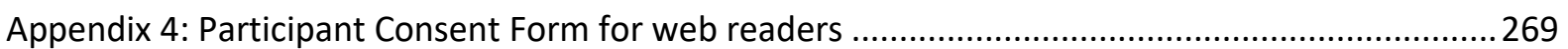

Appendix 5: Interview questions for hotel owners/web deigners ...................................................270

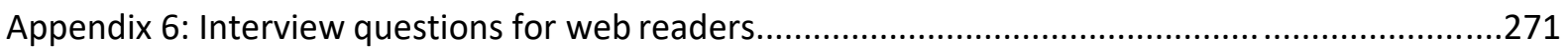

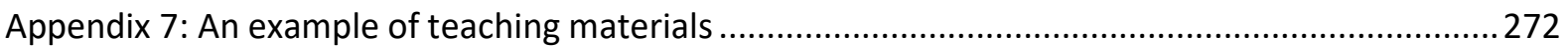




\section{List of Tables}

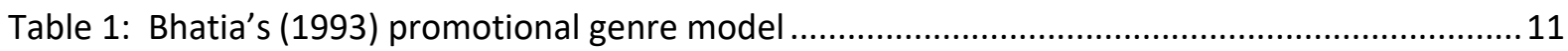

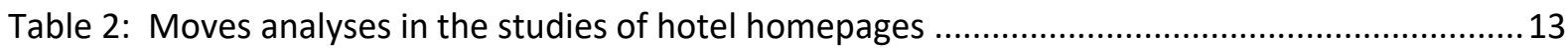

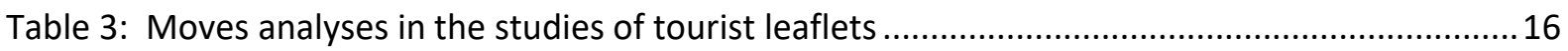

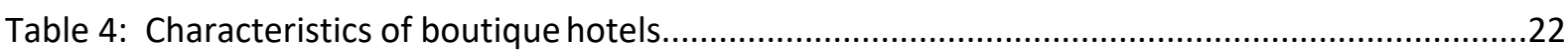

Table 5: an example of a move and steps in scientific research articles ...........................................47

Table 6: Information of the most popular cities in Thailand provided by three different organisations

.

Table 7: Identification of Moves in Thai and New Zealand boutique hotel webpages ........................79

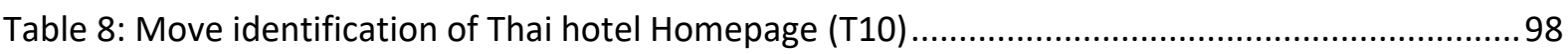

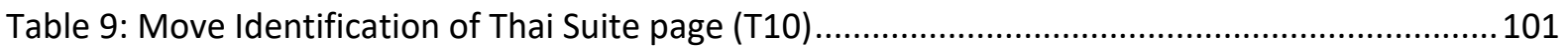

Table 10: Move identification of Thai hotel facilities and Activities page (T10) ................................ 102

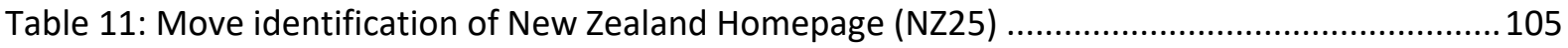

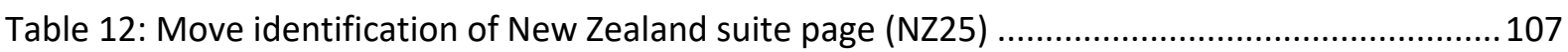

Table 13: Move identification of New Zealand hotel facilities and Activities page (NZ25) ................109

Table 14: The most frequently used imperatives in the Thai and New Zealand datasets.................114

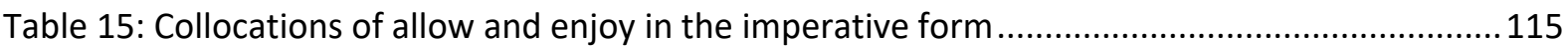

Table 16: Collocations of Book, Click, and Check out in the imperative form ..................................116

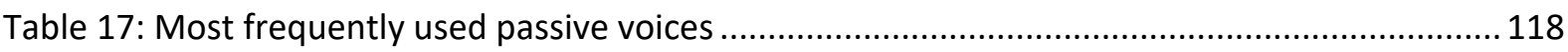

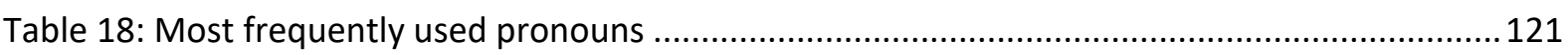

Table 19: Most frequently used modal verbs and their frequency ................................................128

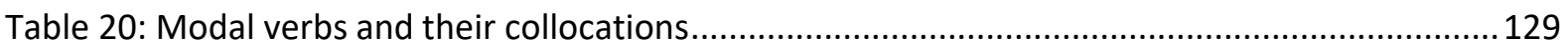

Table 21: Obligatory Moves and one optional move in the Thai and New Zealand dataset.............. 132

Table 22: The frequency and collocations of verbs in Move: (I) in the Thai and New Zealand datasets

Table 23: The frequency of adjectives and collocations in Move: (I) in the Thai and New Zealand

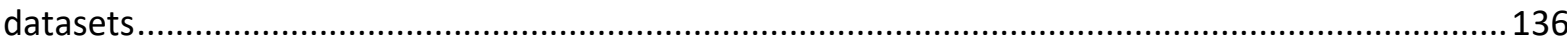

Table 24: The Frequency of pronouns and collocations in Move: (I) in the Thai and New Zealand

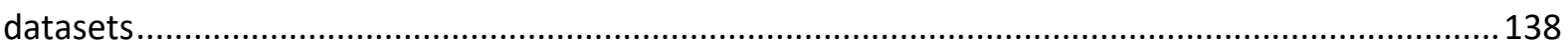

Table 25: The Frequency of verbs and collocations in Move: $(E)$ in the Thai and New Zealand datasets

Table 26: Frequencies of adjectives and their collocation in Move: $(E)$ in the Thai and New Zealand

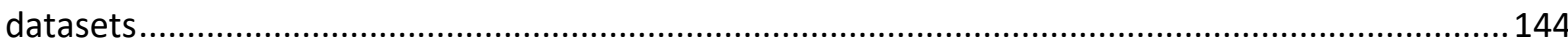

Table 27: Frequencies of pronouns and their collocations in Move: $(E)$ in the Thai and New Zealand datsets.

Table 28: Frequency and collocations of verbs in Move: (DR) in the Thai and New Zealand datasets

Table 29: Frequency and collocations of pronouns in Move: (DR) in the Thai and New Zealand datasets.

Table 30: Frequency and collocations of verbs in Move: (R) in the Thai and New Zealand datasets 160 Table 31: Frequency of adjectives and collocations in Move $(R)$ in the Thai and New Zealand datasets

Table 32: Frequency and collocations of similar adjectives in Thai and New Zealan datasets ..........164 Table 33: Frequency of pronouns and collocations in Move (R) in the Thailand and New Zealand datasets. 
Table 34: The categories of features used in the visual analysis 172

Table 35: The total number of images depicted on the Thai hotel webpages.....

Table 36: The total number of images depicted on the New Zealand hotel webpages

Table 37: The total number of conceptual and narrative images depicted on the hotel webpages in

the Thai and New Zealand datasets....

Table 38: The number of gaze images in the Thai and New Zealand dataset

Table 39: The total number of images showing interactive meaning depicted on the Thai hotel webpage...

Table 40: The total number of images showing interactive meaning depicted on the New Zealand hotel webpages

Table 41: The images showing compositional meaning depicted on the Thai hotel webpages 209 Table 42: The images showing compositional meaning depicted on the New Zealand hotel webpages

Table 45: The use of verbs in the Thai and New Zealand datasets.

Table 46: The use of adjectives in in the Thai and New Zealand datasets

Table 47: The use of pronouns in the Thai and New Zealand datasets. 


\section{List of Figures}

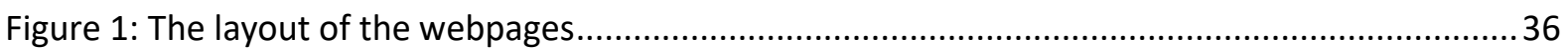

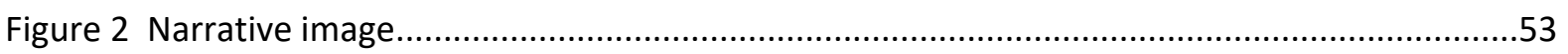

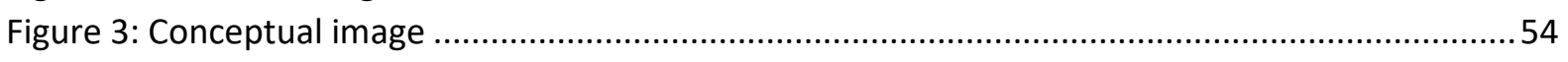

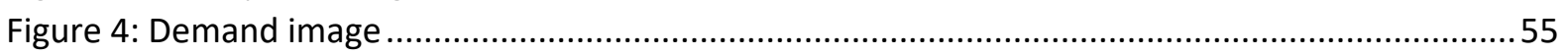

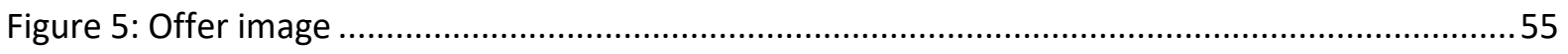

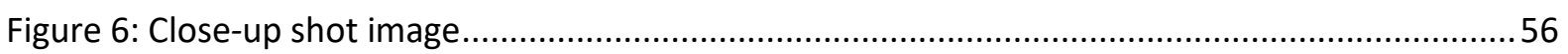

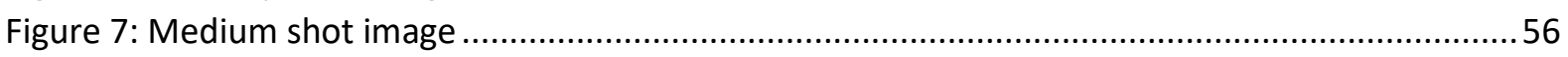

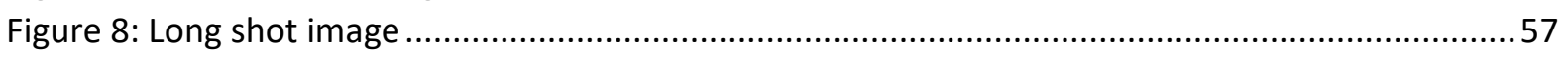

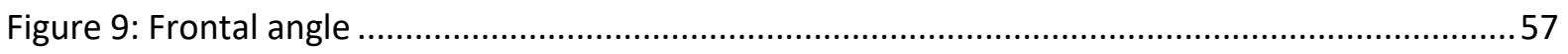

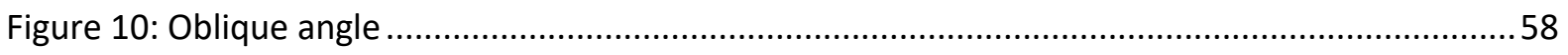

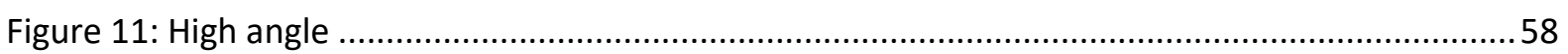

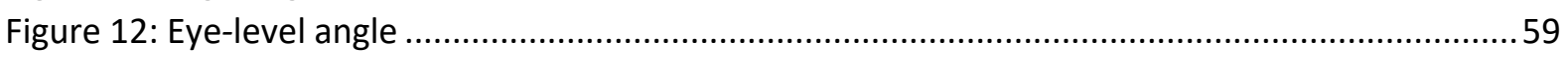

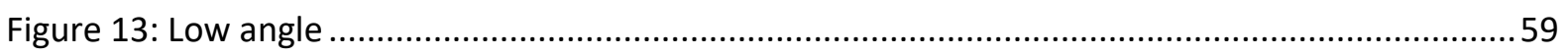

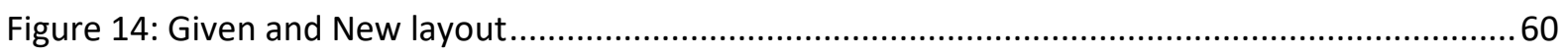

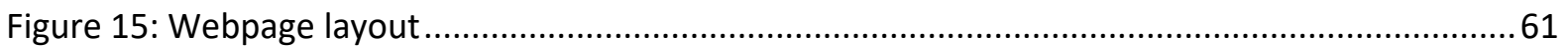

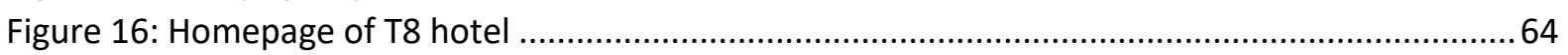

Figure 17: The visual analytical tools adapted from the grammar of visual design (Kress and van

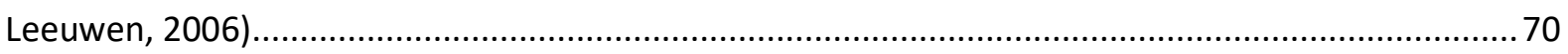

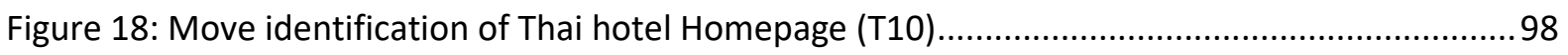

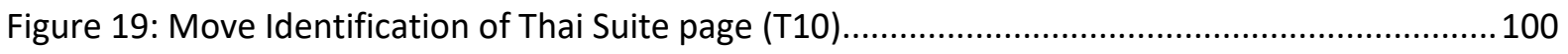

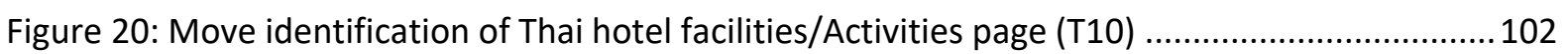

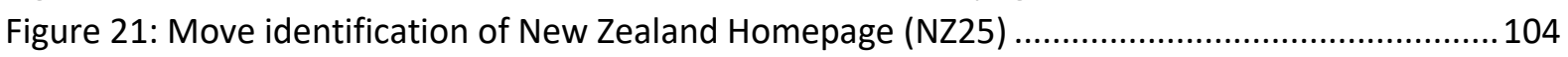

Figure 22: Move identification of New Zealand suite page (NZ25) ................................................ 106

Figure 23 Move identification of New Zealand hotel facilities/Activities page (NZ25) ...................... 109

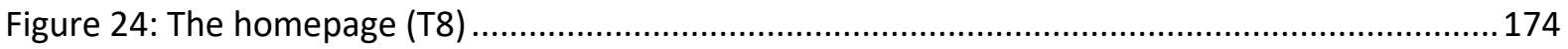

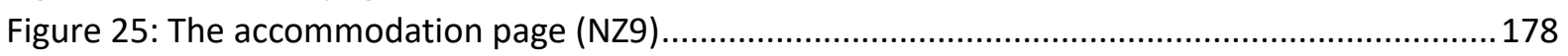

Figure 26: A hotel's exterior and atmosphere (NZ25) ................................................................ 181

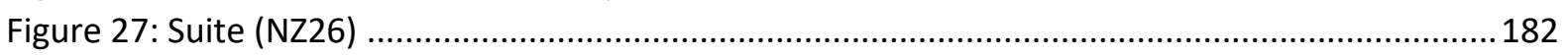

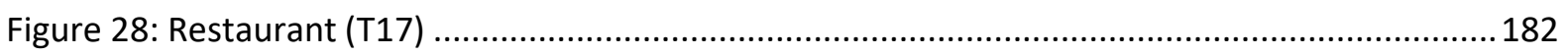

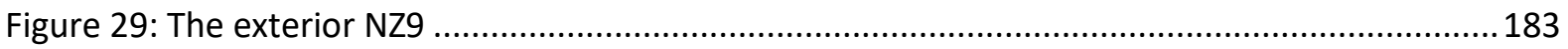

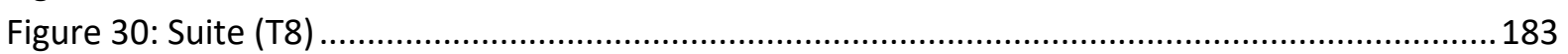

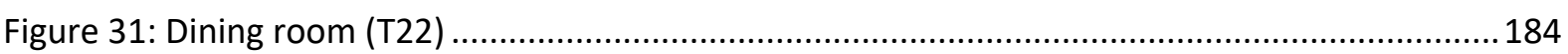

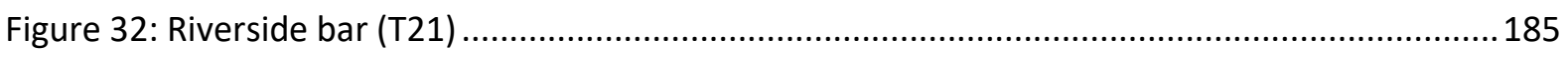

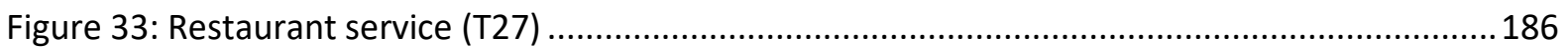

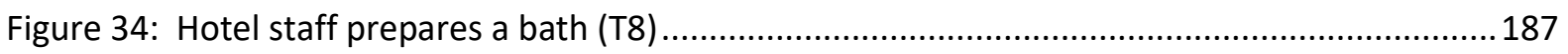

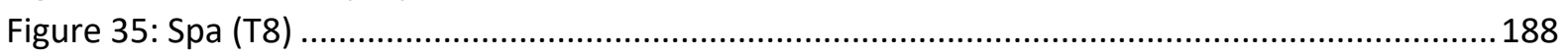

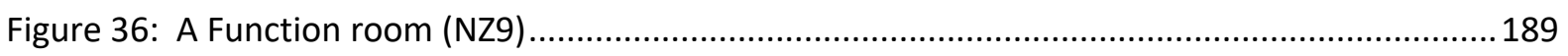

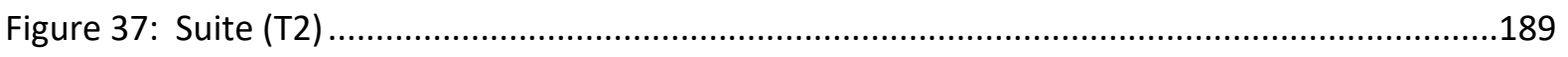

Figure 38: An example of the represented participants found in my datasets ............................... 190

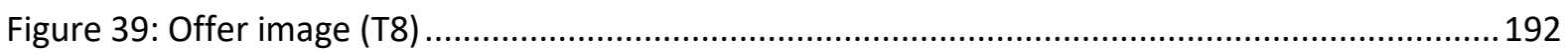

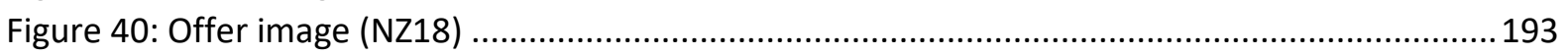

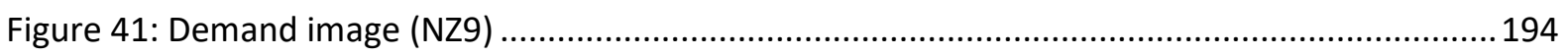

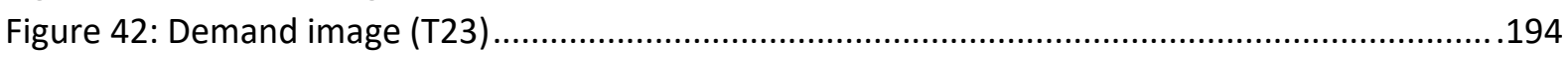




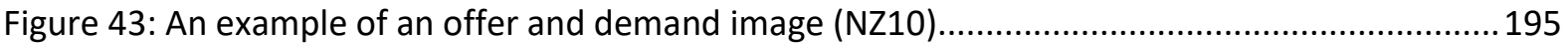

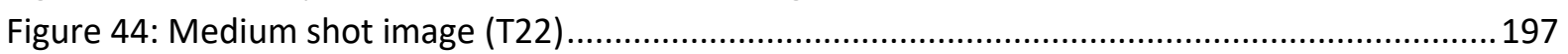

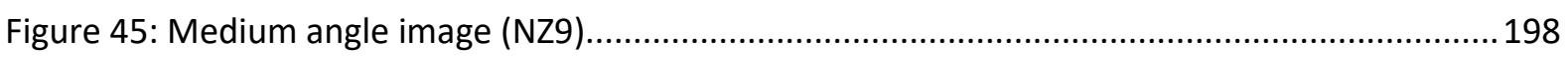

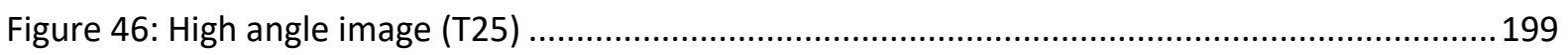

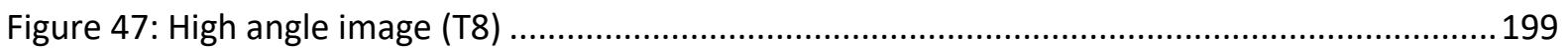

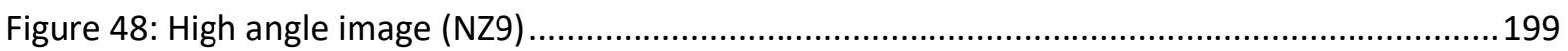

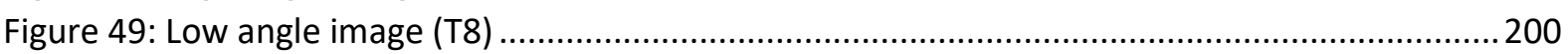

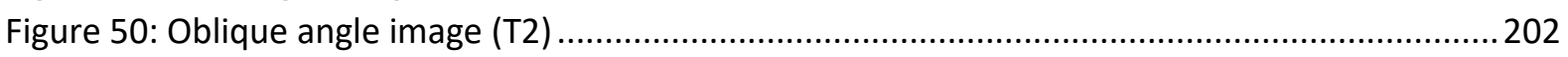

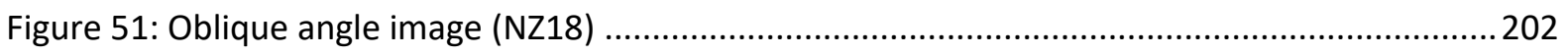

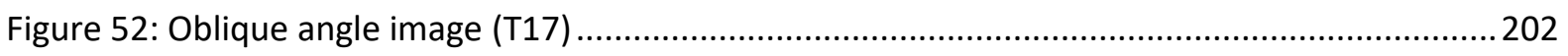

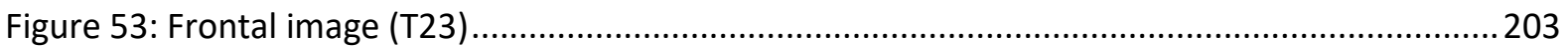

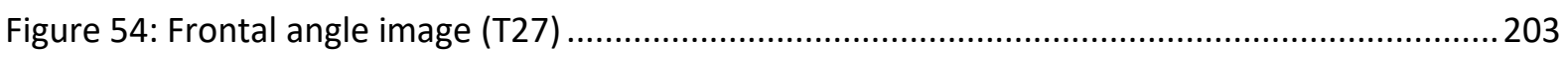

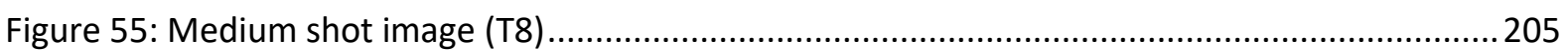

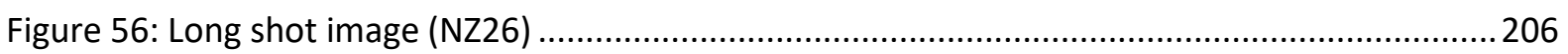

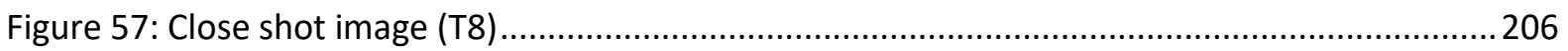

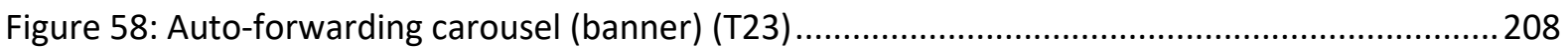

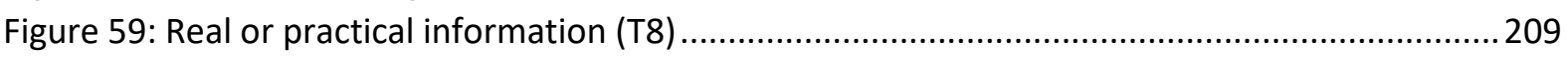

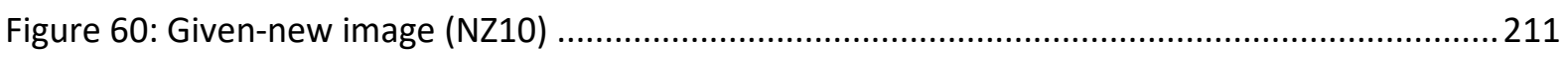

Figure 61: An example of a hotel that does not use given-new structure (T22) ..............................212

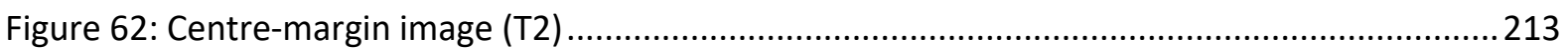

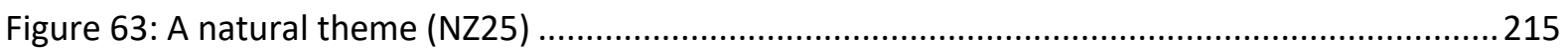

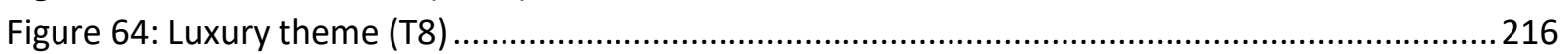




\section{Chapter 1: INTRODUCTION}

This chapter introduces the rationale and background of the study through my teaching experience in English for Tourism and Hotel and the needs of my students. It then moves on to the research questions. The chapter ends with an overview of the thesis.

\subsection{Background of the study}

Web advertising in the hotel industry has been widely adopted as a communicative tool to facilitate and enhance competitiveness in this business. Like other promotional genres, hotel websites use both text and pictures with the principal purpose of eliciting customers' attention. Target guests can easily compare the accommodation, prices, and promotions offered by different hotels. In addition, potential guests can read reviews and take virtual tours to explore the hotels' buildings and their surroundings prior to booking (Rodgers, 2015). Moreover, Tans, the CEO of the online lodging reservation suggested that the Internet brings new opportunities to hoteliers who own small accommodation to advertise their properties to the world (Wiriyapong, 2016).

According to Marco (2002), Medina et al. (2005), and Shepherd and Watters (1999), web advertising is a multi-purpose genre because it contains multiple functions, which include establishing a positive image, informing the readers about existing services, facilities, and products, and persuading them to take action. This is congruent with Bhatia's (2005) description of web advertising as a promotional genre. With the introduction of new technology in mass communication, promotional genres have become more versatile and dynamic in terms of their functions. In order for web designers to communicate these functions and achieve the ultimate goal of persuading the potential customers to buy facilities and services, Bhatia (2005) suggests that the use of innovative forms of language and rhetorical strategies is necessary.

According to the Tourism Ministry, the tourism industry in Thailand is ranked as the fourthgreatest contributor to the country's economy. Additionally, foreign visitor arrivals rose nearly nine percent in 2016 generating 45.9 billion US dollars (Temphairojana, 2017). Such statistics reveal that the travel and hotel industry in Thailand is on the rise. Likewise, Mr. Whiteway, the Director of Skyscanner's Singapore-based commercial department suggested that the rate of online booking is very high in Thailand. This trend is expected to increase dramatically in the next few years (Wiriyapong, 2016).

As English has the status of an international language in the online advertising community, the increasing importance of English in the Thai tourism and hotel industry seems to be consistent 
with the rapid expansion of the industry. Hotel operators advertise a variety of promotional campaigns on their websites to attract the attention of prospective guests from all over the world. In order to promote hotel facilities and services, hotel websites must be written in English to attract international visitors. However, writing effective hotel websites in English is not as easy as it seems. This task is even more difficult for web designers for whom English is not their first language. It requires a high degree of proficiency in English and special training on how to write persuasive online marketing copy, which includes knowledge of language structures, dominant lexicogrammatical features, and organisation of webpages. These issues are of importance in genre analysis, which focuses on the investigation of the characteristics of texts and the social contexts in which the language is used. Genre knowledge, which involves the conventions and structural patterns that are associated with the purposes of the texts, is essential in professional and academic communities. Writers and speakers draw on genre knowledge in order to communicate with members within and outside their communities. Therefore, in order to help novice web designers to produce hotel websites, genre analysis of promotional materials is needed to investigate the characteristics and organisation of this genre. According to Bhatia (1993), "genres are meant to serve the goals of specific discourse communities, and in so doing, they tend to establish relatively stable structural forms and even constrain the use of lexico-grammatical resources in expressing forms" (p. 181). As a result, the knowledge or insights offered by genre analysis help novice writers to produce texts within a particular genre.

The combined necessity of a high level of English competence and knowledge of how to use the generic features and choose visual images to advertise hotel websites is of interest to me because of my experience in Thailand teaching English for Hotel and Tourism. One need identified by my students was developing the ability to create hotel websites in English, as the future intention of many of them included opening a boutique hotel (a distinctive stylishly designed hotel). Their interest in creating a boutique hotel website resulted initially in a student assignment and later motivated me to further investigate the discourse features of these websites. There is very little research on the hotel webpage genre and none to date on boutique hotels, despite their growing popularity and the significance of websites in the hotel industry. Studies have presented a general overview of the appearance and the popularity of the boutique hotel phenomenon (such as Khosravi, Malek, \& Ekiz, 2014; Strannegård \& Strannegård, 2012). However, textual and visual analysis of boutique hotel webpages, particularly cross-cultural analysis, remains limited. Crosscultural aspects are important because culture has been one of the main factors that influences the variation within the genre as proposed by Crossley (2007), Mauranen (2012), and Mur-Dueñas (2013). One study that specifically focuses on genre analysis of hotel homepages is that of Suen 
(2013). In her genre study within the discourse of online advertising, Suen (2013) investigated the language and visual images presented on the homepages of five Star hotels in Hong Kong, including the contextual aspects which shape hotel homepages as a genre. However, Suen (2013) does not pay attention to cross-cultural aspects, focusing mainly on English hotel homepages that are situated in Hong Kong. Few, if any, studies have investigated boutique hotel webpages other than their homepages (e.g., facility pages, suite pages, and restaurant pages).

In addition to the insufficient amount of research into the analysis of discourse structures and the visual design of webpages as a genre for professional development purposes, there are only a few studies available to guide EFL learners in creating hotel websites. Despite the growth of ESP courses in the area of tourism studies, little research has been done in this area to date. Indeed, the use of promotional language and the design of hotel webpages deserves more attention from genre analysts, as there has been no research which focuses on generic features of boutique hotel web advertising as its own genre.

In order to address these issues, I investigated how boutique hotel websites are presented as a genre by using Swales' (1990) and Bhatia's (1993) genre analyses, and Kress and van Leeuwen's visual design analysis model (2006). In regard to textual elements, I examined move structures and the predominant lexico-grammatical features used to achieve genre-specific purposes (register analysis). Apart from textual elements that play an essential role in boutique hotel webpages, visual images are also vital. In order to complete the analysis of boutique hotel webpages as a promotional genre, I explored the layout of website design and how the hotels use visual images to engage with, and persuade the readers. Additionally, I explored why professional copywriters write the way they do.

As language and culture are interrelated, the influences of cross-cultural variation that influence the way the texts are structured are also a research focus (Crossley, 2007; Hyland, 2002; Mauranen, 1993; Vergaro, 2004; Yakhontova, 2002). In order to investigate the cross-cultural variations of boutique hotel webpages, I paid attention to the similarities and differences between the move structures, distinctive linguistic features, and the way that Thai and New Zealand hotels present the images on their webpages. More specifically, the knowledge of genre conventions and visual design that is used to achieve the communicative purposes of hotel online advertising benefits professional as well as academic communities. This knowledge can be used to help novice web designers and novice copy writers, as well as small business owners who aim to open boutique hotels, in the production of hotel websites. In addition to the provision of the knowledge of rhetorical structures and visual images, the interviews with professional web designers and hotel operators about website design processes also offer insights into the development of online 
promotional materials. Apart from the benefit to professional communication, the study also contributes to language pedagogy and further research in ESP. Additionally, these findings provide language descriptions that can be used as teaching material in English for Marketing and English for Hotel and Tourism courses. The details of the significance of the study are discussed further in section 9.3.

\subsection{Research questions}

Based on the above research rationale and objectives, the present study aimed to understand the linguistic characteristics and visual design of boutique hotel webpages. In order to understand the textual structures (moves) and the linguistic resources that the web designers use to realise the purposes of the moves, move analysis and register analysis were conducted. In regard to visual images, I aimed to explore website design and the relationship established between the visual images and the audience. After that, Icompared and contrasted how boutique hotel webpages in the Thai and New Zealand contexts are presented as a genre. To understand the use of the linguistic features and the professional practices of website design of the hotels, I conducted interviews with the hotel owners and experienced web designers.

This study aimed to address the following questions:

1) What are the moves in English boutique hotel websites in Thailand and in New Zealand?

2) What are the distinctive lexico-grammatical features used to serve genre-specific functions?

3) What are the features of visual images in English boutique hotel websites in Thailand and in New Zealand?

4) What are the similarities and differences between boutique hotel websites in Thailand and in New Zealand?

5) What contextual factors influence the variation of the textual and image features in boutique hotel websites in Thailand and in New Zealand?

\subsection{Thesis overview}

This study explores webpages of boutique hotels in Thailand and New Zealand as a genre. Following this introductory chapter, Chapter 2 presents a review of relevant literature of the theories and research on which I based my study. These include a review of boutique hotel and research from different contexts, including genre theory, promotional genre, multidimensional approach, website 
design, and digital discourse. I also indicate the approach to genre I employed in this study. Chapter 3 proceeds to discuss the theoretical approaches I used, which I conceptualise as my principle framework to examine boutique hotel webpages as a genre. Chapter 4 provides a detailed description of the research design and methodology adopted in this study, presenting how I developed my data collection procedure (hotel webpages and participants), and method of data analysis. I used Swales' (1990) and Bhatia's (1993) genre analysis to investigate the macro (move structures) and micro level (lexico-grammatical features) of textual structures of hotel webpages. Kress and van Leeuwen's visual design analysis model (2006) was used to investigate visual images presented in hotel webpages. I used interviews to explore the practices and insights of the experts in the hotel sector.

Chapters 5 to 7 present the main findings of this thesis. Chapter 5 discusses findings relating to the analysis of move structures and the similarities and differences between how boutique hotels in Thai and New Zealand advertise their webpages as a genre. Chapter 6 analyses the register of boutique hotel webpages focusing on personal pronouns, modal verbs, the passive voice, imperatives, and adjectives. Chapter 7 analyses the visual images in each of the twelve hotel webpages, six webpages from each dataset. Chapter 8 discusses the main findings of the study. Chapter 9 summarises the findings with respect to the research questions, providing insiders' insights into the practices of hotel owners and web designers in Thailand and New Zealand. It also offers directions for future research, methodological contributions as well as professional and pedagogical applications of my study. 


\section{Chapter 2: LITERATURE REVIEW}

This chapter reviews past literature on four major topics related to the present study. The first section of this chapter focuses on genre theory, which is the main theoretical framework of this study. This part deals with three main schools of genre with an emphasis on English for Specific Purposes. Subsequently, the chapter proceeds to tourism and hospitality research in the boutique hotel industry. The next part pays attention to visual analysis approach which has been applied in the tourism and hotel context. The last part of this chapter explores website design and digital genre.

\subsection{Genre Theory}

Traditional conceptions of genre focused on textual regularities (Freedman \& Medway, 1994, p.1), typified forms of discourse (Tardy, 2013 p. 54), and linguistic realization (Flowerdew, 2002, p. 91). These originate in the repeated uses by the members of the community, and later on , these uses become conventional. Traditionally, genres were viewed as an approach to classifying types of literary texts that are static in nature (Adam \& Artemeva, 2002; Freedman \& Medway, 1994; Johns, 2002). Scholars attempted to identify and classify them into their exclusive categories by considering solely their surface features or form and content (Adam \& Artemeva, 2002; Freedman \& Medway, 1994; Johns, 2002). However, this concept of genres was challenged by scholars such as Bakhtin (1984), who view genres not as traditional classification schemes but rather as utterances or typical forms of utterances (Bakhtin, 1986, p.63), or social activities (Vergaro, 2004, p. 2004), which are shaped by the conventions (Johns, 2002, p. 3) of the community. The idea that genres are socially developed and connected to a particular community has been supported and amplified by many genre theorists. For example, Bakhtin (1986) and Hyland (2002) maintain that utterances are socially bounded and connected to a speech community. Flowerdew (2002) also argues that genres are not only textual structures but rather the production of a speech community because genres are shaped by the values and practices within that community. Other genre theorists whose view is that genre is socially bounded are Pare and Smart (1994). These scholars define genre as a distinctive event of regularities across four dimensions: a set of texts, the way the texts have been created, reading practices employed to interpret the texts, and the social roles performed by writers and readers. They also suggest that recurrent patterns in the structure, rhetorical moves and styles of texts are easily and obviously observable aspects of genre. That is to say, genres are represented by their regular sequence, in which their purpose and function can be observed and identified. 
This new notion of genres has recently influenced the reconceptualisation of genres by integrating the idea that genres are social action into the traditional concept of genre. The current concept of genre has become more complex because it is combined with social contexts and community value (Freedman \& Medway, 1994; Johns, 2002). The reconception of genre has shifted away from categorising texts based on surface level to focus more on contextual aspects, where the genre is situated. As a result, the focus of genre analysis has changed from the language itself to language in use, which is shaped by community members.

There are three major perspectives to genre analysis - English for Specific Purposes (ESP), Rhetorical Genre Studies (RGS), and Systemic Functional Linguistics (SFL). The boundaries between each tradition of genres have been gradually shifting and now overlap. However, there is some distinction between these three school of genres in terms of their focus as well as research approach.

ESP is based on Swales' genre analysis theory (Swales, 1990). Swales (1990) defines genre as texts belonging to a genre sharing common communicative purposes, which is recognised by the members of a discourse community, and which constrains structure, style and content of texts in the genre. With their primary focus on the benefit of genre in language learning, the fundamental principle of ESP is to describe rhetorical structures as well as lexico-grammatical features, and to explore a relationship between lexico-grammatical features of a genre and the functions that they perform. My study is most closely aligned with this school.

My study also draws on RGS. This genre tradition is based on the work of Miller (1984) who pays attention to relations between text and social contexts in which genres are situated rather than the linguistic features of the genres. The underlying principle of RGS is that genre is a form of social action. The theorists in this school focus on the investigation of the social and cultural contexts in order to understand how genre is used to achieve their actions and purposes (Fakhruddin \& Hassan, 2015). Since the primary focus of this school is more on situational contexts than on rhetorical patterns or linguistic features, the majority of research in this school relies on ethnographic approaches with an attempt to provide thick descriptions of contexts, where genre occurs. In terms of pedagogical application, since the RGS approach is not linguistic like ESP, several theorists in this strand do not agree with teaching writing genres because they believe that teaching genres represents an inauthentic context for understanding writing (Freedman, 1994). In addition, the research studies in this school aim to help university students and novice professionals understand the social functions of genres (Hyon, 1996). 
The third school in genre studies is SFL, pioneered by Halliday $(1985 ; 1994)$. Halliday's theory emphasises the relationship between language and its context of use. Similar to Rhetorical Genre Studies, SFL also perceives genre as a social process since genres are used to achieve social actions (Hyon, 1996). Research in this camp has applied the Hallidayan model of linguistic analysis to examine textual patterns (Christie, 1991; Hyon, 1996). In essence, according to SFL and ESP, linguistic properties are highly connected to situational contexts and functions. As a result, both schools adopt a linguistic framework to investigate and describe genre according to their functional features or communicative purposes (Fakhruddin \& Hassan, 2015). Even though ESP and SFL focus on teaching outcomes, the ESP approach adopts a more various set of pedagogies and their aims are to respond to the needs of non-native speakers of English in academic and professional contexts. On the other hand, SFL focuses on pedagogy at primary and secondary schools as well as adult migrant English education and workplace training programs.

The fundamental similarity between these three traditions of genres (RGS, SFL, and ESP) is that genres and contexts are interrelated. Only the study of recurring linguistic features does not contribute to the overall understanding of genres (Bawarshi \& Reiff, 2010; Johns, 2002). However, the approaches used to analyse genres within these three schools differ. Since genres in ESP are realised primarily by their communicative purposes, ESP genre analysts focus on identifying moves and linguistic features of genres. The key principle of SFL is to investigate textual structures that are connected to the social function of a genre; and RGS pays attention to social contexts and the purposes of the members in a speech community (Feez, 2002). The differences between these three schools of genres have resulted in different approaches to the ways the genres are realised, studied and applied in pedagogical and professional contexts (Fakhruddin \& Hassan, 2015). The detailed discussion of these three traditions of genres is on chapter 3: Theory chapter. The next section discusses the importance of a genre-based approach.

\subsubsection{Importance of a Genre-Based approach}

The genre-based approach, with its main emphasis on communication purposes (Henry \& Roseberry, 1998), arose from the pioneering work of Halliday (1985), Swales (1981; 1990), and Bhatia $(1991 ; 1993)$. In essence, genre-based teaching focuses on discovering what students need to achieve in their writing class and exploring what students usually do when they write (Hyland, 2004). From the 1970s, this genre approach has been increasingly adopted to facilitate writing in genrebased pedagogies (Hyland, 2007). The widespread use of the approach in the classroom is also supported by Johns $(2002$, p. 3$)$, who views that the focus on genre is a major paradigm shift in teaching literacy. This is because a genre-based course, in providing detailed and explicit 
descriptions of the ways language is structured in a particular situational context, can act as a valuable resource for teachers and learners (Hyland, 2003; 2004; 2007). In addition, these conventions gradually will build a configuration of background knowledge that the students can apply in their writing in other situational contexts such as business letters and application letters (Rahman, 2011).

The genre-based approach also enables teachers to be more aware and respond to the writing needs of their students, who are from diversified cultural background, and to elaborate on the benefits of genre pedagogies (Hyland, 2004; 2007). Christie and Martin (1997) and Hyland (2004; 2007) claim that the genre-based approach enhances students' writing skills by providing them with straightforward writing formulas and a context to reflect and understand how the language patterns create meanings. In genre-based classrooms, teachers provide explicit writing structures and patterns and also explain why the texts are structured the way they are. This is because genre-based teaching includes both linguistic patterns and the contextual situation where the language descriptions are used to achieve the communicative purposes of the genre (Ning, 2008). In this respect, Ellis, Johnson, Henry and Roseberry (1998) conducted a study to investigate whether an EAP/ESP genre-based classroom enhances success in learning experiences compared to a non-genre classroom. As expected, their findings clearly show that focusing on generic structures of texts enabled the students to achieve their writing objectives and improve their motivation in writing. Genre analysis and genre-based approach have mainly been adopted for pedagogical purposes in order to provide language descriptions for second language learners. However, my study suggests that genre analysis can be also used to benefit professionals outside academia. My analysis of textual elements, visual images, and interview data can help to provide basic guidelines for novice web designers or hotel owners of how to write boutique hotel webpages.

\subsubsection{Limitations of Genre-Based approach}

Despite the fact that the genre pedagogies have been valued as effective and successful strategies for writing, they have been challenged and questioned by a number of scholars (Feez, 2002; Francesconi, 2012; Kay \& Dudley-Evans, 1998). The major concern is that the genre-based approach embodies the force of prescriptivism which inhibits students from having the freedom and creativity to develop their writing skills. This is because the students are bound by the formulas and conventionalities provided. Francesconi (2012) argues that the notion of generic integrity (p. 22) is important in a genre-based teaching approach because it shows recognisable patterns that are the inherent properties of genre. These standardised structures allow the members of the discourse community to realise that these notable patterns belong to the same genre. However, Freedman 
(1993) and Hyland $(2004 ; 2007)$ disagree with the concept of conventionalities. They explain that the conventionalities are useful only if they occur in their context but when the contexts are removed the models become meaningless. As a result, the students are not able to learn the models. The risk of prescriptivism can be minimised if teachers are aware of this issue and know how to integrate models and rules in the classroom properly. The following section discusses the background and main focus of promotional genres, which my study is mainly aligned.

\subsection{Hotel and tourism material advertising as a promotional genre}

In addition to academic discourses, genre analysis is also adopted in the study of other nonacademic genres. The promotional genre is one of the examples of non-academic genres whose characteristics and organizational patterns have been investigated using move analysis. The examples include sales promotional letters (Bhatia, 1993), tax computation letters (Flowerdew \&Wan, 2006), brief tourism information texts (Henry \& Roseberry, 1998), application letters (Henry \& Roseberry, 2001; Bhatia, 1993), and restaurant reviews (Hou, 2012). These documents are categorised as the same genre because they have the same communicative purposes (Bhatia 1993), which include informing and persuading audiences to buy products, services, or ideas (Bhatia, 2005).

The promotional genre is one of the most pervasive genres because its persuasive value has increasingly influenced the function of other non-promotional discourses such as academic and political discourse (Bhatia, 2005). For example, since research articles are persuasive in nature, they adopt certain discourse structures and lexico-grammatical features that are also used in promotional genres. Bhatia (2004) also confirms that it is common to see similar features of discourse across disciplinary boundaries.

The three primary functions of the promotional genre are to inform, persuade, and remind target audience (Mill \& Morrison, 2002) to buy products or services (Bhatia 1993 \& 2005; Kathpalia, 1992). The most distinctive characteristics of promotional discourse, which are commonly found in the form of advertisements, are the use of innovative linguistic resources, textual organisations (move structures), and rhetorical strategies (steps) (Bhatia, 2005). This is evidenced by studies on move structures and linguistic devices (e.g., Fuertes-Olivera, Velasco-Sacristán, Arribas-Baño, \& Samaniego-Fernández, 2001; Labrador, Ramón, Alaiz-Moretón \& Sanjurjo-Gonzále, 2014; Zhou, 2012).

Similar to other promotional texts, hotel webpages are also constructed with the same communicative purpose of promoting hotels and persuading the audience to use their facilities and services. This communicative purpose can be realised by the use of recurring move structures, and stylistic conventions that advertisers employ to accomplish their communicative goals. In 
advertising, persuasion plays an important role in getting a reaction from the audience. Similarly, other types of discourses such as political speech (Virtanen \& Halmari, 2005) also adopt persuasion to achieve their communicative purpose. This communicative purpose can be observed by the use of certain persuasive devices such as imperatives and the we inclusive pronoun.

Due to the specialised characteristics of promotional discourse, several studies have been conducted to explore how copywriters construct language and its structures to create a persuasive effect (Bhatia, 1993; 2005). Drawing on the move analysis proposed by Swales (1990), Bhatia (1993) further develops the genre analysis model in an attempt to expand the scope of genre studies from academic into professional genres as well as to provide a thick description of language in use. Drawing on his move analysis of a sales promotional letter, Bhatia (1993) proposed a model for structural description in promotional genres. Even though this move analysis framework is based on only one example of sale promotional letter to Standard Bank, it has been widely adopted in several studies. The following are the move structures proposed by Bhatia (1993).

\section{Table 1: Bhatia's (1993) promotional genre model}

\section{Rhetorical moves}

Establishing credentials

Introducing the offer

Offering incentives

Enclosing documents

Soliciting response

Using pressure tactics

Ending politely

Table 1 shows the move analysis framework proposed by Bhatia (1993). In line with the value of persuasion (Bhatia, 2005) discussed in the previous section, the primary purpose of this letter is to persuade readers to buy the products and services. There are seven moves that the writer uses to achieve their communicative purposes. This section focuses on the detail of these seven move structures.

The first move is Establishing credentials, which is used to assure the readers that their requirements are recognised and the company is expert in the business and can fulfil those needs. In addition to highlighting the company expertise of the business, this move also emphasises the achievement of the company. The persuasive devices that the writer uses to achieve this communicative purpose in the letter were the use of the pronoun we and you as well as the use of the expression "We are expertly aware...." 
The second move is Introducing the offer. The main purpose of this move is to promote the products and services, which is the most important part of sales letters (Bhatia, 1993). The writer introduces the key features of the products/services and their important information such as the price, the date of availability, and the benefits of the products/service that are related to the potential customers' needs. In order to promote the products and services to the readers, writers often use modifiers to create product evaluation and linguistic resources such as the following "We are pleased to bring to your attention/to offer/announce/introduce..."

The third move is Offering incentives. This move is an optional move in Bhatia's framework because he suggests that Offering incentives seems to be culturally related. Bhatia (1993) further explains that based on his experience with the business community, this marketing strategy tends to be more common in Singapore than Western communities. This move: Offering incentives is used after introducing the products/services to the potential customers. The writers adopt this move to attract the readers' attention by offering incentives or a special discount.

The next move is Enclosing documents. Even though this move is extensively used by certain companies, it is only an optional move. This is because it depends on the company's marketing strategies. It is usually used at the end of the letter. The fifth move is Soliciting response, which is an obligatory move. It is not only used to encourage the readers to initiate further contact with the company but is also used as a polite ending. This move is achieved by an extensive use of politeness markers and an offer of free service. The sixth move is Using pressure tactics, which usually appears at the end of the letter. It is an optional move, in which the main purpose is to urge the customers who are interested in the products to take a prompt decision to buy the products. The last move is Ending politely. It is an obligatory move, which is used to close the letter.

Bhatia (1993) notes that the principal communicative purpose of a promotional genre is to persuade customers to buy products or services. Even though these seven moves are often cited and adopted in advertisements in order to achieve persuasive value and the main communicative purposes of describing and selling products/services, the patterns of occurrence of these moves are usually not fixed but are varied, which reflect the distinctive characteristics of advertising (Bhatia 2005). These subtle distinctions may be influenced by a sensitivity or creativity of writers to the contextual aspects, where effectiveness of the genre is valued (Bhatia, 2004; Bhatia, 2005; Virtanen \& Halmari, 2005). However, these variations still remain within the boundary of the genre.

Like other promotional texts, hotel websites are developed with the communicative purpose of promoting hotels and persuading the audience to use their facilities and services. Textual organisation (moves) and characteristic linguistic patterns are the rhetorical tools that writers employ to accomplish their communicative goals. Drawing upon Swales's (1990) genre analysis and 
Bhatia's (1993) promotional genre analysis, several studies have been conducted to identify the main communicative purposes, move structures and distinctive linguistic features of hotel and tourism promotional materials. This includes the investigation of hotel chain homepages (Akkhakraisi, 2004); three-star hotel homepages (Klankanna, 2007); serviced apartment homepages (Phongsuwan, 2009); and five-star hotel homepages (Suen, 2009; 2013).

Genre studies of hotel advertising have been investigated in two main aspects; move analysis, and linguistic features. Studies by Akkhakraisi (2004), Klankanna (2007), Phongsuwan (2009), Suen (2009) have adopted Bhatia's framework to analyse promotional online text. Consequently, there are certain similarities of text organisation and linguistic features among these studies, which will be discussed below.

Table 2: Moves analyses in the studies of hotel homepages

\begin{tabular}{|c|c|c|c|c|}
\hline & Klankanna (2007) & Akkhakraisi (2004) & Phongsuwan (2009) & Suen (2009) \\
\hline $\begin{array}{l}\text { Context of the } \\
\text { study }\end{array}$ & $\begin{array}{l}\text { three-star hotel homepages } \\
\text { in Thailand }\end{array}$ & $\begin{array}{l}\text { hotel chain homepages in } \\
\text { Thailand }\end{array}$ & $\begin{array}{l}\text { serviced apartments } \\
\text { homepages in Thailand }\end{array}$ & $\begin{array}{l}\text { five-star hotel } \\
\text { homepages in Hong } \\
\text { Kong }\end{array}$ \\
\hline $\begin{array}{l}\text { Description of } \\
\text { moves. }\end{array}$ & $\begin{array}{l}\text { Move 1: Attracting attention } \\
\text { Move 2: Establishing } \\
\text { credentials } \\
\text { Move 3: Introducing the } \\
\text { offer } \\
\text { Move 4: Soliciting response }\end{array}$ & $\begin{array}{l}\text { Move 1: Attracting attention } \\
\text { Move 2: Calling for action } \\
\text { Move 3: Describing Specials } \\
\text { Move 4: Indicating terms and } \\
\text { conditions (optional) }\end{array}$ & $\begin{array}{l}\text { Move 1: Identify apartment } \\
\text { Move 2: Greeting } \\
\text { Move 3: Introducing } \\
\text { apartment } \\
\text { Move 4: Calling for action } \\
\text { Move 5: Giving contact } \\
\text { information } \\
\text { Move 6: Giving information }\end{array}$ & $\begin{array}{l}\text { Move 1: Identifying } \\
\text { the brand } \\
\text { Move 2: Introducing } \\
\text { the content list of the } \\
\text { hotel website } \\
\text { Move 3: Establishing } \\
\text { contact } \\
\text { Move 4: Establishing } \\
\text { credentials } \\
\text { Move 5: Introducing } \\
\text { accommodation }\end{array}$ \\
\hline
\end{tabular}

From the above- mentioned studies, the most salient aspects are that they are categorised in a promotional genre which mainly aims to advertise their hotels and the researchers have adopted Bhatia's framework to analyse promotional online text. Consequently, the moves and steps in these studies are likely to share the same name and features. For example, Move: Attracting attention occurs in two studies (Akkhakraisi, 2004; Klankanna, 2007). Additionally, there are two studies 
(Klankanna, 2007; Suen 2009) that directly borrowed the names of moves from Bhatia's 1993 promotional genre analysis model: Establishing credentials and Soliciting response.

Additionally, it is found that distinctive linguistic features, namely personal pronouns, modal auxiliary verbs, adjectives, imperatives and complex noun phrases are mostly employed to advertise hotel features and facilities in the various hotel homepages. These studies found that an effective way to engage the audience is by using personal pronouns like you, yours, we, and our.

There are certain limitations of the previous studies. Firstly, the researchers ignore the importance of ethnographic methods in studying how web designers write. They pay attention only to move structure and grammatical features. As a result, they are not able to provide an insiders' perspective toward how they use the genres. Only one study (Suen, 2013) integrates ethnographic approach into her study of hotel homepages. Ethnographic research enables her to gain a deeper understanding of the hotels' practices. For example, interviews with two web designers and a hotel practitioner revealed that there was a change in hotel website presentation. In the past, hotel websites were like traditional brochures. On the other hand, the present website designs paid more attention to design and interactive features.

Furthermore, it is believed that even though the conventionalised structuring of genre is determined by its communicative purposes, intercultural variation also plays an important role in the rhetorical preferences of writers within a specific genre. No comparative study has been conducted to compare two datasets of the same genre, which are written by copywriters who have geographically and culturally different backgrounds in order to investigate whether cultural aspects play a significant part in genre study or not.

Also, the above studies claim that their analysis can benefit pedagogy, as the results of the study can be used as a guideline for ESP writing classes for the students who attempt to acquire information about how to write hotel homepages. In addition, Crossley's (2007) claim that the concept of move analysis should be taught to $L 2$ learners is true as it might contribute to more effective production of texts. Nevertheless, no study provided teaching materials that showed a clear method of how to write a hotel homepage. In my study, I provide teaching material related to genre analysis of hotel webpages in Appendix 7.

Similar to the above studies of hotel homepages, the genre analysis framework has been widely adopted in the tourism sector. These studies investigate tourist leaflets (Boonchaayanant, 2003; Henry and Roseberry, 1996; Mongkholjuck, 2009; Sinraksa, 2009). Even though these studies contain similar research purposes of identifying organisational patterns and prevailing lexicogrammatical features which are associated with the occurrence of moves in tourist leaflets, the contexts where these tourism materials are produced and distributed are different. These differences 
may influence the patterns and numbers of moves. Interestingly, the findings of the following studies show that the contexts where the tourist brochures occur can influence the variation of the occurrence of moves which further confirms that promotional genres are fluid and dynamic. Table 3 following shows the finding of move analysis of the tourist leaflets from different studies. 
Table 3: Moves analyses in the studies of tourist leaflets

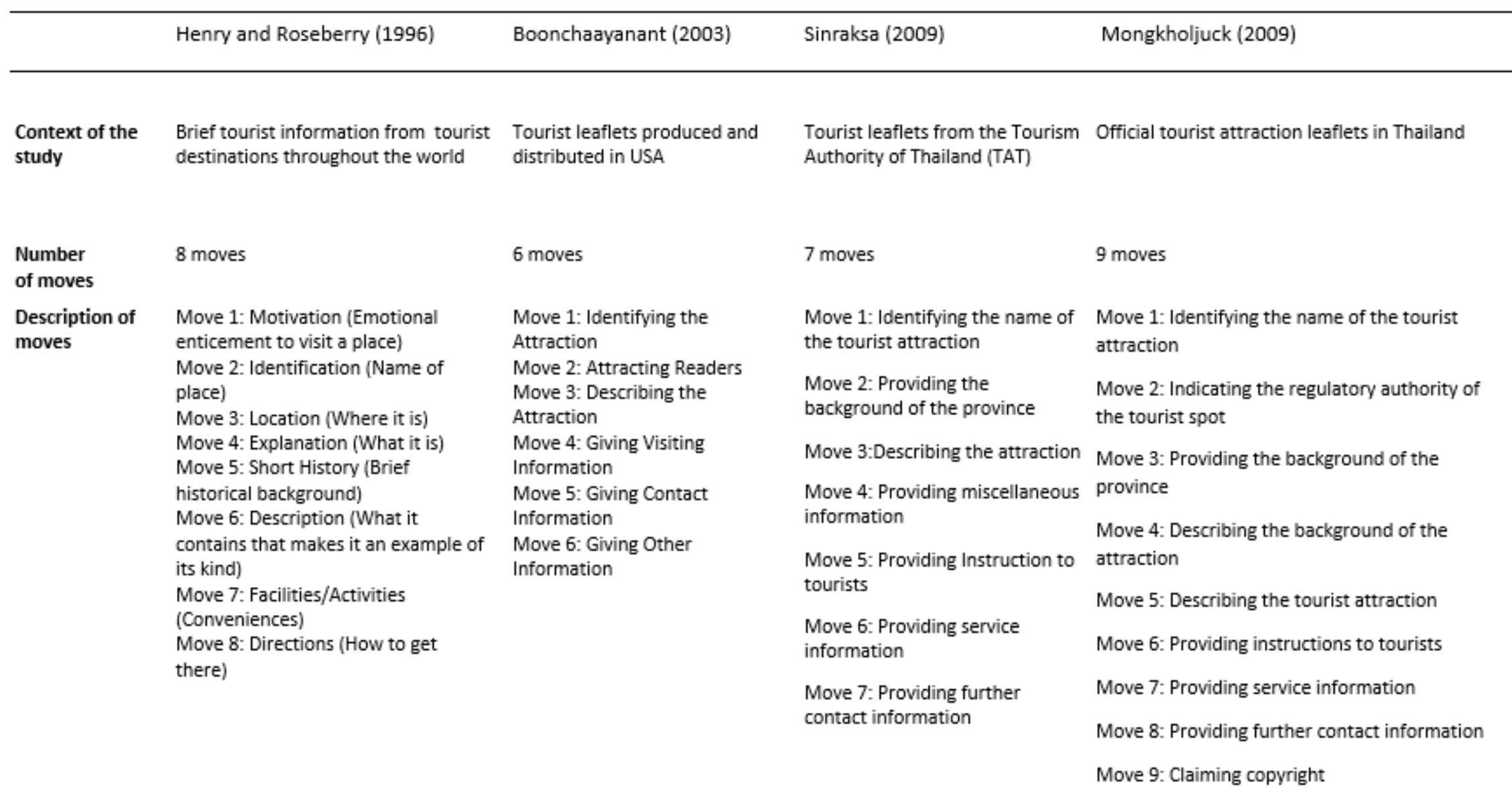


Table 3 lists the moves that occurred in the tourist leaflets produced in different contexts. Even though the move structures of these tourist materials are not identical, there are no significant distinctions between them. That is to say, the number of moves varies between studies but they share similarities in terms of move structure. There are certain moves that occur in every study, which indicates that these moves are the main communicative purposes of the tourist leaflets. The examples include Move: Identifying the attraction and Move: Describing the tourist attraction. To introduce the place to the tourists, Move: Identifying the attraction is adopted. In order to describe and promote the place that the tourist will discover while visiting the place, copywriters use Move: Describing the tourist attraction (Mongkholjuck 2009). As a result, the fundamental purposes of tourist leaflets do not function only to give information but also to persuade the readers to come to visit the attraction. These findings are consistent with Bhatia's (2004) suggestion that informative genres are often integrated with persuasive value. Since move analysis has no explicit rules, move demarcation is based on the comprehension of the text and the linguistic markers of move boundaries. It is likely that the move analysts may rely on their intuition when analysing moves and steps. This subjectivity can cause variations in move structures and the number of moves as shown in the studies above (Tisapramotkul, 2007). However these variations still maintain the identity of tourist leaflet genre.

Apart from the subjectivity in move demarcation by move analysts, which can cause variation and diversity in defining move structures, the aspects of cultural differences play an important role in genre. This is because in professional communication, we usually carry our local cultural constraints with us even when we communicate with people from different cultures. The notion of the impact of cultural differences on schematic structures of a text is emphasised by Crossley (2007), Hyland (2000), Mauranen (1993), Yakhontova, (2002), Vergaro (2004). They further claim that genres are socially realised by the members in a discourse community and are governed by certain conventions showing that they belong to a particular genre. Different cultures may produce different expectations in terms of linguistic choices with the result that authors are hardwired to produce texts (Mauranen, 1993). Mauranen (1993) also further explains that the term cultural differences can apply to either national culture or disciplinary culture, where rhetorical variation can occur within a genre or at country or culture level (p.5). Relevant to Mauranen (1993), Crossley (2007) confirms that moves are culturally dependent; when a writer travels between cultures but remains within a similar genre, the move expectation of that genre may change. This differs from the opinion expressed by Bhatia (1993) who suggests that texts that are structured and classified in the same genre are likely to have same overall communicative purposes. Regardless of the nationality of the members in a community, they tend to use move structures and linguistic 
resources within a broad range of typical conventions. In my study, I test Bhatia's (1993) claim, in that conventions of genres influence the regularities in writing of the members in a discourse community.

The relationship between culture and discourse structures seems to be deemphasised by Bhatia (1993). Bhatia (1993) is aware of the influence that the local culture of the writers has on their writing, but claims that this cross-cultural variation is less severe in the academic genre than in the professional genre, since academic writing is more rigid and conforms to the conventions of rhetorical structures.

Within the cross-cultural variation in the promotional genre, only a few studies have been conducted to examine the importance and influence of cultural background of discourse structures and the use of linguistic features (macro and micro structures). As a result, the relationship between cross-cultural variation and genre analysis in the promotional genre, especially in the hotel and tourism context remains to be investigated.

\subsection{Tourism and hospitality research in boutique hotel context}

This section discusses tourism and hospitality studies related to boutique hotels, which includes the definition of boutique hotels and important attributes of boutiqueness. The development of boutique hotels in the 1980s was influenced by attempts to move away from conventional large chain or box hotels in order to respond to the desire from niche market travellers to have different experiences (Aggett, 2007; Munsters \& de Klumbis, 2006; Rogerson, 2010). The first boutique hotel (design or lifestyle hotel) was London's Blake Hotel which originated in 1978 (Chan, 2012; Jones, Day, \& Quadri-Felitti, 2013). However, it was not until the 1990's that the boutique hotel phenomenon started to appear on a large scale and gained its popularity among travellers who sought sophisticated experiences and intimate service by staying at an innovative form of hotel. The changing trend from the traditional hotels to boutique hotels has drawn attention from several researchers (e.g., Aggett, 2007; Chan, 2012; McIntosh \& Siggs, 2005; Rogerson, 2010; Tidtichumrernporn, 2012) who aim to scrutinise boutique hotel situations in a variety of countries.

Due to its growing demand and the need to differentiate themselves from traditional hotels, the term boutique hotel is employed by many hotel establishments. There have been attempts from industry professionals and researchers to construct a conventionally agreed upon definition for boutique hotels and their characteristics in different parts of the world. However, the difficulties in acquiring a standardised definition of boutique hotel still remain. The problem in developing a formal definition of boutique hotel is discussed for example by Rogerson (2010) in relation to South Africa's official grading system of the hotels. The grading system is problematic since they do not 
have a clearly defined category for boutique hotels. Rogerson (2010) explains that many hotels in South Africa were concerned that their uniqueness was not considered important based on the grading system. Country houses or guest houses are able to claim themselves as boutique hotels; accordingly, it can contaminate the image of the boutique hotel sector as a whole. The example of the situation of boutique hotels in Africa echoes a wider need to acquire an agreed upon definition of boutique hotels to avoid harm to the boutique hotel image.

Similar concern that the definition of boutique hotel is not agreed upon is found in a number of studies (e.g., Aggett, 2007; Chan, 2012; Covelli, 2005; Hartesvelt, 2006; Jones, Day, \& QuadriFelitti, 2013; McIntosh \& Siggs, 2005; Mckenney, 2015). However, according to Lim and Endean (2008), one possible explanation of this issue is that there are some differences in the defining characteristics of boutique hotels between academics and practitioners, which includes location, size, facilities, and star rating.

As a result of the fact that there are no agreed-upon categories defining boutique hotels, the term boutique hotel has been liberally employed by many hotel operators, which then causes confusion and wrong perceptions by tourists who are looking for a truly personalised service and homely ambience from real boutique hotels (Lim \& Endean, 2008; Rogerson, 2010). Standardising the official definition of boutique hotels will be greatly profitable for many sectors for example: tourists, boutique hotel operators, and organisations who are responsible for rating hotel quality. Since many boutique hotel operators are being disadvantaged from an absence of clarity and definition (Mintel 2011), many operators urge the development of an official definition because they are aware of its significance. They believe that the clarity of the definition may help them to maintain the true image of a boutique hotel and prevent deliberate misuse by hotels, who falsely position themselves as genuine ones (Lim \& Endean, 2009; Rogerson, 2010; Mintel, 2011). This issue also occurs in my dataset where there appears to be several hotels marketing themselves as a boutique hotel regardless of what qualities they manage to deliver to the guests.

Even though there is no official definition of boutique hotel that sets the boutique establishment apart from traditional lodgings, many organisations and practitioners (e.g., Aggett, 2007; Covelli, 2005; Hartesvelt, 2006; Jones, Day, \& Quadri-Felitti, 2013; Khosravi, Malek \& Ekiz 2014; McIntosh \& Siggs, 2005; Mckenney, 2015; Rogerson, 2010) are attempting to establish a specific definition of boutique hotel based on the characteristics that the hotels have in common. In order to identify the characteristics of boutiqueness, many studies have conducted interviews (Jones, Day, \& Quadri-Felitti, 2013; Khosravi, Malek \& Ekiz 2014; McIntosh \& Siggs, 2005), or employed questionnaires (e.g., Aggett, 2007; Lim \& Endean, 2009) to collect data from boutique 
hotel operators and/or hotel guests. For example, the study of McIntosh and Siggs (2005) drew on interview data from boutique hotel operators and travellers in New Zealand. This data revealed five dimensions required to maintain a boutique hotel experience. These dimensions are unique character, personalised, homely, quality, and value added (P.77). In addition, McIntosh and Siggs (2005) noted that the relatively small-scale dimensions seem to be specific to boutique accommodation and may not be applicable to other kinds of accommodation.

Apart from the five key attributes of boutique hotels proposed by Mclntosh and Siggs (2005), various other studies (e.g., Aggett, 2007; Covelli, 2005; Hartesvelt, 2006; Mckenney, 2015; Roger, 2010) have also investigated the characteristics of boutique hotel. Even though these studies focus on boutique hotel in different countries, they have shared a basic set of key properties that attract consumers to boutique accommodation: size (small scale: ranging from 20-150 rooms), architecture and design both inside and outside the hotels, individuality and uniqueness, specialised and personalised service, individual guest attention, intimacy, high-tech facilities and value added (local knowledge that the guests can get from the hotel hosts).

However, Timothy and Teye (2009) offered a different point of view of boutique hotels. They do not focus on the important attributes of uniqueness of boutique hotels but considered mainly how boutique hotels are established. They categorised a boutique hotel establishment as a general bed and breakfast. Timothy and Teye (2009) indicated that boutique accommodation is the ubiquitous bed and breakfast type (p.178). Inns, homestays, pensions, and guesthouse are also put into this classification. This type of accommodation refers to private or semi-private properties, where the guests usually share the space with the owners of the properties. In addition, the properties are small, ranging from 1 to 20 rooms. Generally, the characteristics and architectural design of this type of accommodation vary according to the country and region. For example, in the United Kingdom most bed and breakfasts are considered budget accommodation, while in the United States, Canada, and New Zealand, this kind of lodging is more sophisticated and expensive. Most of them have full facilities, distinctive character, and historical background (ibid, p. 179). Timothy and Teye's criteria for categorising hotels are relatively loose. As a result, certain characteristics of small boutique accommodation in New Zealand overlap and are similar to bed and breakfast accommodation that Timothy and Teye (2009) described. This is because boutique hotels share two common features (small scale and distinctive design) with the other small hotels in Timothy and Teye's (2009) category. In addition, many of them are converted from bed and breakfast establishment by adding more facilities and sumptuous decoration to attract tourists who would like to experience properties with a unique character. So far in this section, I have discussed 
different views of boutiqueness based on previous literature that attempts to construct a unified definition of boutique hotel. Even though Timothy and Teye (2009) offer a contradictory concept of boutique hotel, their definition of boutiqueness shares key properties with other studies such as unique features and small size.

In addition to the key properties mentioned above, there appears to be a new alternative form of boutique hotel or the adaptive use of old buildings (McIntosh \& Siggs, 2005, p. 217). This type of hotel focuses on conserving and redeveloping historical, cultural, and heritage locations where hotel operators attempt to refurbish old buildings to transform them to boutique hotels in order to attract tourists who have special interest in historical or cultural sites (Henderson 2011; Henderson, Liew, Ong \& Quek, 2013). However, by interviewing guests, McIntosh and Siggs (2005) found that the conversion from old buildings to boutique accommodation is not appealing to some respondents. Their findings revealed that one major disadvantage of the old houses is a lack of comfort and facilities.

These unique representations of boutique hotels are considered an influential factor motivating travellers to stay at boutique hotels. In order to make the characteristics of boutique hotels based on previous studies easy to visualise, I summarise them as follows. The terms that they used to define boutiqueness may be different across these studies but their explanation of terms is relatively similar. These include Unique character (McIntosh and Siggs, 2005) and Distinctiveness (Hartesvelt, 2006). Although these two studies used different terminology, their meaning and interpretation of these two terms are similar. Both terms are used to refer to the uniqueness of physical setting, architectural design, atmosphere, and services represented by boutique hotels. 
Table 4: Characteristics of boutique hotels

\begin{tabular}{|c|c|c|c|c|c|c|c|c|}
\hline $\begin{array}{l}\text { Characteristics } \\
\text { Of boutique } \\
\text { hotels }\end{array}$ & $\begin{array}{l}\text { Anhar } \\
(2001)\end{array}$ & $\begin{array}{l}\text { Mclntosh } \\
\text { and Siggs } \\
\text { (2005) }\end{array}$ & $\begin{array}{l}\text { Aggett } \\
\text { (2007) }\end{array}$ & $\begin{array}{l}\text { Khosravi, } \\
\text { Malek } \\
\text { and Ekiz } \\
(2014)\end{array}$ & $\begin{array}{l}\text { Hartesvelt } \\
(2006)\end{array}$ & $\begin{array}{l}\text { Götz } \\
(2015)\end{array}$ & $\begin{array}{l}\text { Wang } \\
(2013)\end{array}$ & $\begin{array}{l}\text { Jones, } \\
\text { Day, \& } \\
\text { Quadri- } \\
\text { Felitti } \\
\text { (2013) }\end{array}$ \\
\hline Uniqueness & $x$ & $\mathrm{x}$ & $x$ & & $x$ & $x$ & $\mathrm{x}$ & $\mathrm{x}$ \\
\hline Intimacy & $x$ & & & & $x$ & & & \\
\hline Location & $x$ & & $x$ & $x$ & $x$ & $x$ & $x$ & \\
\hline $\begin{array}{l}\text { Historic related } \\
\text { design }\end{array}$ & $x$ & & & & & $x$ & & $x$ \\
\hline $\begin{array}{l}\text { Architecture } \\
\text { and design }\end{array}$ & $x$ & & & $x$ & & & $x$ & \\
\hline $\begin{array}{l}\text { Provision of a } \\
\text { variety of } \\
\text { entertainments }\end{array}$ & $x$ & & & & & & & \\
\hline Technology & $x$ & & & & & & $x$ & \\
\hline Personalised & & $x$ & $x$ & & & $x$ & $x$ & \\
\hline Value added & & $x$ & & & & & & \\
\hline Quality & & $x$ & $x$ & & & & & \\
\hline Homely & & $x$ & & & & & & \\
\hline $\begin{array}{l}\text { Service } \\
\text { provided }\end{array}$ & & & $x$ & $x$ & $x$ & & & \\
\hline Room features & & & & $x$ & & & & $x$ \\
\hline Small scale & & & & & $x$ & $x$ & & \\
\hline $\begin{array}{l}\text { Small number } \\
\text { of guests }\end{array}$ & & & & & $x$ & & & \\
\hline $\begin{array}{l}\text { Provision of } \\
\text { restaurants }\end{array}$ & & & & & $x$ & & & \\
\hline High profit & & & & & $x$ & & & \\
\hline Individual & & & & & $x$ & & & $x$ \\
\hline $\begin{array}{l}\text { Providing } \\
\text { social spaces }\end{array}$ & & & & & & & & $x$ \\
\hline
\end{tabular}

Table 4 shows that although the definition of boutique hotel and its describing attributes appear to be varied among these researchers, the majority of boutique hotels generally agree on the terms and identification of some common boutique hotel characteristics. These features cover uniqueness, personalised services, and location. From table 4, uniqueness is the most appreciated feature across eight studies.

\section{Uniqueness or unique characteristics}

Owing to the desire of boutique hotels to differentiate themselves from a block design and experience of established chain hotels, uniqueness is one of the most crucial attributes that enhances the success of boutique hotels. Unique characteristics have been found less controversial 
among previous studies. Distinction in architectural design, cosiness, and intimacy are fundamental properties (Munsters \& de Klumbis, 2006; Olga, 2009; Vithal \& Gillespie, 2011). Previous studies reveal that one of the most renowned approaches to creating identity and uniqueness appears to be buying old historic buildings and transforming them into boutique properties with a history (Chang \& Teo, 2009; Hartesvelt, 2006; McIntosh \& Siggs, 2005).

\section{Location}

Most studies indicate that location is one of the most fundamental domains that guests consider before they make a decision to make a booking (Aggett, 2007; Anhar, 2001; Götz, 2015; Hartesvelt, 2006). Anhar (2001) advanced a comprehensive view on the importance of location. She explained that the two primary points of destination that enhance the success of boutique hotel are: city and resort location. Apart from an ease of transportation and convenience of the location, the trendiness and chic-ness of the local community are also important (Anhar, 2001; Olga, 2009). In addition to city location, also prominent is resort location where the target guests expect to have intimate experiences from the facilities and services offered by boutique hotels. Anhar (2001) also suggested that the ideal location for a successful boutique resort is secluded. However, Hartesvelt (2006) argued that location is not a fundamental reason for guests. Visitors can generally manage their own transportation to visit the hotels of their choice.

\section{Personalised service}

Personalised service is favoured by many of the boutique hotel guests as a motivating factor in their decision to visit boutique properties (Aggett, 2007; Anhar, 2001; Götz, 2015; Mclntosh \& Siggs, 2005). Apart from the uniqueness of architectural design, the success of a boutique hotel is also driven by the offer of tailor-made services by hosts/staff members (Callan \& Fearon, 1997; Munsters \& de Klumbis, 2006). Munsters and de Klumbis (2006) suggested that the relationship between hotel staff and guests is the key to success because boutique hotel guests have high expectations from the services provided. Hotel staff members are well trained to be able to match the guests' expectation and experiences. Furthermore, McIntosh and Siggs (2005) agreed that successful personalised services can be achieved not only by the services themselves but also by special care, personal touch, and friendly interaction between the guests and the hosts.

\section{Size}

The size of boutique accommodation appears to be a controversial issue in the the boutique hotel sector because this term creates confusion and distorts the meaning of boutique hotel. According to Anhar (2001) and Hartesvelt (2006), the maximum number of rooms that boutique 
hotels have should not be more than 150 in order to maintain intimacy between hotel guests and hotel staff members. However, the idea that boutique hotels should be small scale has been argued against by lan Schrager, the founder of lan Schrager hotels which provides a total of 3,000 suites across his nine hotels (Anhar, 2001). In line with lan Schrager, Mckenney (2015) also noted that size is controversial as there is a considerable variation in the between a numbers of suites across boutique properties. For instance, 3,000 suites in Marriots' Autograph collection compared to 15 rooms in The Cosmopolitan Las Vegas. In addition, Ian Schrager argued that the core quality in maintaining boutiqueness is not the size but architectural design and a provision of entertainment for the guests (Anhar, 2001). The above discussion reveals that the argument over size is contested throughout the boutique hotel industry. This is due to the fact that there is a lack of clarity and standardised definition to control the practice of the boutique hotel industry. Many chain hotels can deliberately take advantage of the term boutique hotel to benefit themselves.

\section{Value added}

Despite the fact that value added is relatively new and has not been touched upon in the boutique hotel literature, it is becoming more important in the boutique hotel sector. This augmented attribute is an influential criterion that persuades guests to make a reservation (McIntosh \& Siggs, 2005). According to McIntosh and Siggs (2005), value added includes local knowledge and extra entertainment in the neighbourhood. Value added is demanded by a group of potential guests who are interested in art and local culture. This group of guests would like to have an additional experience such as interacting with locals (Freund de Klumbis, 2005; Mclntosh \& Siggs, 2005). In line with McIntosh and Siggs's (2005) idea of value added, Munsters and de Klumbis (2006) confirmed that local culture and cultural attractions are the main components for boutique hotels to attract tourists' attention.

As outlined above, previous studies have investigated the important attributes and particular situations of boutique accommodation in various countries, but none of them has examined how boutique hotels advertise themselves in the market and position themselves through the language that they use on their hotel website. In order to address these issues, I investigate how boutique hotel websites are presented as a genre because knowledge about genre conventions enables novice writers to understand the conventions of the discourse community (Bhatia, $2004 \mathrm{p}$. 185). 


\subsection{Visual analysis approach}

Visual images in online advertising play a crucial role in stimulating desire in the target audience. They attract the readers' attention, promote product features and arouse emotion (O'Guinn, Allen, \& Semenik, 1998). I consider hotel websites as an example of multimodal discourse due to the combination of textual features and visual design. In addition to the analysis of textual elements, this present study also examines how web designers present visual images in hotel webpages in terms of the position and relationship created between images and the visual images. With regard to visual analysis in tourism and hotel discourse, two of the most common approaches that are widely adopted to explore visual elements in tourism and hotel texts are content analysis and multimodal analysis. However, these two approaches are developed from different theoretical backgrounds and their main research foci are also different. The purpose of this section is to review the literature relating to research in content analysis and multimodality discourse analysis (henceforth MDA) with a particular focus on the hotel and tourism, promotional discourse, as well as web-based advertising. The first section and second sections outline the background and definition of these two major approaches and provide my justification for the selection of the visual analysis approach that I used to answer my research questions. The third section focuses on three main approaches in the multimodal domain, with a particular emphasis on Kress and van Leeuwen's (1996 \& 2006) framework, which forms the basis of my visual image analysis and related studies that are relevant to this framework.

\subsubsection{Content analysis}

This section discusses the notion of content analysis and related studies in which content analysis was adopted to examine visual elements depicted in the compositions. Content analysis originated as a quantitative analysis approach with an emphasis on identifying and capturing specified features within a text or composition (Franzosi, 2010). However, the scope of the studies using content analysis is mainly limited to simple data coding and categorising (Hsieh \& Shannon, 2005). Examples of these studies include tourist brochures (Dann, 1996), hotel advertisements in China (Siu and Fung, 1998), the cultural content of websites (Singh, Zhao \& Hu, 2005), and children's media (Herbozo, Tantleff-Dunn, Gokee-Larose, \&Thomson, 2004). Although some of these studies (e.g., Dann, 1996) provide qualitative analyses, they are still limited to categorisation and descriptive/interpretative explanation. The scope of analyses does not go beyond categories and statistical frequency. 
Theoretically, simple word counts can reflect the underlying meaning of a text. This underlying value is derived based on the systematic observation of a certain features/patterns from texts where each quality is generally used to formulate a coding category (Franzosi, 2010, p. 549550) or variables (Bill 2001, p. 13). According to Franzosi (2010), there are a variety of methods which are frequently adopted in content analysis such as word counts in syntactic analysis, referential analysis, prepositional analysis, and thematic analysis. Firstly, syntactic analysis is a fruitful technique to draw beneficial information from a composition. For example, the study of the pronoun I in two articles written by two different journalists showed that the pronoun I was found 13 times in the article written by L'Aurore while it did not appear in L'Humannite. This can explain the differences in ideology between two articles.

Franzosi (2010) indicates that simple word counts can reflect the underlying meaning of a text based on the systematic observation of a certain qualities from texts where each quality is used to formulate a coding category (p. 549-550). Franzosi (2010) also points out that a coding scheme performs its role as a questionnaire where each coding category serves a specific research question. The most effective way to begin conducting content analysis is to understand the research questions and then make a decision about which approach can answer those questions. In addition, a coding scheme that contains a list of valid and distinguishable variables is considered as important as the reliability of a coding process in content analysis, which can be systematically evaluated by utilising inter-coder reliability.

The next approach that is commonly used in content analysis is thematic analysis, where a categorisation is used as the foundation of the coding scheme in order to identify the predominant themes presented in a text. However, Franzosi (2010) notes that capturing themes is not an easy process since a coding scheme should be sensitive enough for the researchers to pick up the themes that they aim to explore from a text.

A third approach, referential content analysis is an approach which is generally used to capture the complexity of language in the production of meaning (Franzosi, 2010, p. 551). This approach is used to discover how an underlying value is communicated. Franzosi (2010) exemplified the references by portraying the character Mr. Schlumpf in the two articles "L'Aurore" and "L'Humannite" as having a negative quality through the reflection of the attributes of the words depraved, complaisant, and criminal. Referential analysis exhibits the ideological aspect or attitude of the writer.

In my study, I adopted content analysis to examine and characterise the characteristics of visual images depicted in boutique hotel webpages. The purpose of using content analysis was to 
minimise the size of the dataset that I used for move analysis and my visual image study. This approach involved counting and categorising the number of facility images depicted in suite pages of boutique hotel websites to retrieve representatives from the whole population. The next section discusses examples of studies in promotional discourse that adopt content analysis to explore the content of media, advertisement, and hotel brochures and websites.

In an initial study, Bell and Milic (2002) used a combination of content analysis and semiotic analysis to investigate gender stereotypes in 827 advertisements in popular Australian magazines. Drawing on Goffman's (1979)work, where he suggests that women's roles are portrayed as subordinate and infantilised in advertisements, Bell and Milic (2002) adopted Kress and van Leeuwen's visual analysis framework in addition to a content analysis approach to explore whether visual semiotic notions can be distinguishably identified as variables and used as the basis of quantitative content analysis. The results showed that the portrayal of female and male participants was relatively different, a finding which corresponded with Goffman's (1979) propositions. The most interesting part in Bell and Milic's (2002) study is the integration of semiotic and content analysis that the authors adopted to construct the variables to test Goffman's (1979) hypotheses. However, the interpretation of the findings is based on the categories that the authors have formulated. In my study, I used content analysis to classify visual images presented on boutique hotel webpages for data analysis. This is because content analysis does not provide qualitative descriptions to answer my research questions, which aim to explore the persuasive effects of the visual images and the interaction between the images and the viewers.

In line with Bell and Milic's (2002) study, Ford, Voli, Honetcutt, and Casey (1998) used content analysis to investigate gender roles in 452 Japanese magazine advertisements. They established nine variables (such as product, sex, age, and location) related to differences in gender representation. The coders examined the ads and classified them according to gender traits defined such as active, confident, strong (male traits) attractive, cute, and delicate (female qualities). The chi square results revealed that there were significant differences within gender and age categorisation. The results also suggested that the representation of female values in past advertisements has changed from negative values to more positive attributes in present ads. The content analysis findings provide gender characteristic categories, which enable the researchers to draw the description of gender status depicted in the advertisements.

In a later work using content analysis, Herbozo, Tantleff-Dunn, Gokee-Larose, and Thompson (2004) explored body image-related messages in 25 popular children's videos and 20 books for children aged 4-8 (e.g., Cinderella, Peter Pan, and The Little Mermaid). The results revealed that 
there was a highlight of physical stereotype represented in several videos but it was less significant in books. In this study, content analysis was used to investigate 16 variables in reference to body image-related messages. For example, one attractive male has a slim and muscular body, at least one female figure is thin, and evil characters have unattractive physical features. After that, three undergraduate psychology students were trained to evaluate the dataset and report on the message exhibited in the sample. The findings of this content analysis revealed that the notions of beauty and thinness are more emphasised in children's videos than children's books. The primary indicator of beauty of the female characters is physical attractiveness, especially thinness (p. 30). The portrayal of positive features of the characters in the videos was associated with being more friendly, gentle, and successful. On the other hand, fat characters were frequently represented as having evil or undesirable qualities.

Apart from the application of content analysis to magazines and children books, Maynard and Tian (2004) investigated 100 top global brands advertised online in China to explore how the presentation of the top brand products adapt to different local markets. The variables were 1) a cooperative relationship with local government, 2) an award or honour given by the Chinese government or local community, and 3) a contribution to local entertainment. The results suggested a notable level of localisation in online advertising in China. The findings revealed that 58 out of 100 top global brands provided a Chinese language option in the websites and presented local events and awards or honours offered by the Chinese government or local community.

Consistent with the above study, Govers and Go (2005) explored the identity of visual and textual structures in twenty Dubai-based websites through content analysis. In regard to the visual element analysis, 505 images were categorised according to their appearance and themes that are commonly adopted in tourism research. The findings showed that the presentation of the tourism websites failed to reflect the cultural identity of Dubai. The most distinctive tourism theme focused on presenting facilities and activities (e.g., dining experiences or experience modern shopping facilities).

The above discussion shows the main three content analysis approaches proposed by Franzosi (2010) and related studies. Even though content analysis has been widely adopted in promotional discourse, it is not relevant to my research focus. This is because I did not use content analysis to test hypotheses or establish variables but rather for my data selection procedure. I used it to categorise the images in boutique hotel websites based on their features of representation (facilities in hotel suites). These classifications enabled me to select websites for my visual analysis. 
That is to say, the selection was based on the numbers of images and the categories that appear in each website.

\subsubsection{Multimodal analysis approach (MDA)}

MDA was originally developed from social semiotic theory, Systemic Functional Linguistics (SFL). This approach concerns how participants construct the possible meanings of semiotic resources (e.g., text, image, and sculpture) in social contexts to produce communicative events and to understand them (Jewitt, 2009b; van Leeuwen, 2005; O'Halloran, 2008; O'Halloran, 2013). Even though MDA has been conceptualised with some differences from SFL linguists, mostly, they share a similar focus. These linguists are interested in investigating the integration of the linguistic domain with other resources, which contributes to the overall meaning of the visual compositions. Specifically, O'Halloran (2013) refers to MDA as the study of language in conjunction with other elements, such as images, scientific symbolism, gestures, action, music and sound. Likewise, Jewitt (2009a) notes that MDA pays attention to a set of approaches that are used to understand communication and representation beyond texts. It covers a wide range of interactional forms (e.g., image, gesture, gaze, and posture and the relationship between these visual elements). Similar to O'Halloran (2013) and Jewitt (2009a), Kress and van Leeuwen (2001, p. 20) define MDA as "the use of several semiotic modes in the design of a semiotic product or event." This definition means that MDA is related to my study because hotel websites are examples of multimodal discourse as they carry features of both text and images. Drawing on Kress and van Leeuwen's framework "The Grammar of Visual Design" (2006), my study investigates how web designers present visual images in hotel websites in terms of organisation, position, and relationship between the viewers and the images.

Kress and Van Leeuwen (2006) provide an interesting theoretical basis for understanding visual images. The framework of Kress and van Leeuwen (2006) focuses on three major metafunctions: Representational meaning, Interactive meaning, and Compositional meaning. Representational meaning refers to the fact that visual images depict participants which may be human, animal, place or object. These participants may perform actions and/or interact with each other to create meaning in the visual images (Unsworth 2001, Jewitt \& Oyama, 2001). On the other hand, Interactive meaning focuses on imaginary relations between visual elements and viewers of an image that occur through their interaction (Jewitt \& Oyama, 2001). In order to analyse the degree of interactive meaning in the visual images, there are three aspects - gaze, size of frame and perspective - that should be observed. The last meaning is compositional meaning. This is characterised by the integration between three interrelated systems: information value, salience, 
and framing (Kress and van Leeuwen, 2006). The detail of these three meanings are discussed in Chapter 4: Methodology.

Drawing from the theories of visual grammar proposed by Kress and van Leeuwen (1996; 2006), an increasing number of studies in multimodal discourse have been conducted to explore visual images in different multimodal contexts (e.g. newspapers, children's books, magazine advertisement, brochures, tourism and hotel online advertisements). However, this section mainly reviews the multimodal literature in promotional discourse because it is related to my study context. In this sense, I pay attention to magazine advertisements, tourism and hotel brochures, and online advertisements. As Kress and van Leeuwen's $(1996 ; 2006)$ framework is very comprehensive, the studies discussed below adopt different aspects of this influential framework in order to suit their specific purposes. I mainly pay attention to the areas that are relevant to my study. Focusing on how the research in the promotional discourse adopts Kress and van Leeuwen's framework in their study provides me with comprehensive guidelines and expands my perspective of how to interpret visual compositions in promotional discourse.

Following Kress and van Leeuwen's (1996; 2006) approach, Hiippala (2007) applies the three metafunctions: Representational meaning, Interactive meaning, and Compositional meaning to tourist brochures advertising the city of Helsinki. However, I focus here on the analysis of representational function of the visual resources, which I use as a guideline in my comparative analysis between Thai and New Zealand datasets. Hiippala's (2007) study shows that this approach is helpful to provide a comprehensive explanation of how images can communicate and reflect the society and lifestyle in Helsinki. In regard to the representational role, the depiction of the visual elements shows a change in the brochure theme between the 1970s and 1990s. The main focus of the brochures has changed from depicting the traditional tourist sites to the places that are associated with a variety of examples of the Helsinki lifestyle.

In line with Hiippala's (2007) study, Francesconi (2011) investigates images in a tourist brochure in Malta. The framework of Kress and Van Leeuwen (2006) is adopted to identify what the visual images aim to convey, the relationship between the visual resources and the readers, and how the visual images are organised in the brochure. Based on representational meaning or what is represented in the brochure, the brochure does not highlight the main value of hospitality, which is often created by a representation of the interaction between locals and tourists. In this study, no images represent activities and scenes that show the engagement between local people and tourists. Instead, the main focus of the brochure, as seen in the recurring images, seems to be the cultural value of Malta's heritage. In terms of interactive meaning, most pictures adopt an eye-level 
angle, which signifies the equal status of the viewers and the images. In this context, Francesconi (2011) suggests that eye-level images represent good hospitality and a warm welcome from locals. In regard to compositional meaning, an interesting aspect is the use of light and colour, where the study says the use of different colours and lights connotes the different mood and feeling of the images to the viewers.

Another important study, which pays attention to the multimodal construction represented in brochures, is Rajandran (2009). He uses Kress and Leeuwen's framework (1996; 2006) in analysing bank brochures to explore how the bank uses visual images to create a relationship with the viewers. With respect to Rajandran's (2009) study, I mainly pay attention to how he describes Interactive meaning to describe the relations between the participants in visual images and the audience. Rajandran (2009) explains that visual images create two kinds of imaginary relations between characters and viewers by the use of demand and offer images. According to Kress and van Leeuwen (2006), demand images are those where human characters have eye contact with viewers in order to establish an imaginary relationship with them. By using this kind of image, the viewers are invited to engage interpersonally with the characters. On the other hand, offer images represent characters who do not have eye contact with viewers, which connote disengagement and a distant relationship between the characters and the viewers. Rajandran's (2009) study reveals that the bank brochures apply only offer images, depicting the characters smiling and sitting relaxed with friends as if they are living in their own world without paying attention to viewers. Even though the bank uses only offer images, they make the products more desirable for the viewers. In addition, it is unlikely that the bank intends to create a distant relationship with the audience. However, Rajandran (2009) explains that the images depicting happy and relaxed people create a favourable effect for the banks to establish their credibility. The bank uses the brochures as a strategy to influence the viewers' perception about the good value of the products.

Apart from the analysis of visual images represented in the brochures, Kress and van Leeuwens' framework has also been expanded and widely used in digital discourse, which is evidenced by recent publications (e.g., Harvey 2013: commercial hair loss websites; Lemke 2002: NASA webpages; Maci 2007: tourism homepage; and Suen 2013: hotel homepage). One of the most relevant studies in online promotional discourse is Suen (2013). She explores the visual resources depicted on Hong Kong five-star hotel homepages. The visual images are analysed in terms of the representational, interpersonal and compositional aspects. Based on representational meaning defined by Kress and van Leeuwen (2006), there are two kinds of images narrative and conceptual images. According to Kress and van Leeuwen $(2006$, p. 59) narrative pictures are realised by a vector 
emanating from participants, who are depicted as performing roles or "doing something to or for each other." To realise whether the images are considered as narrative image or conceptual image, the representation of vectorial relations between the participants is the main indicator. When participants or characters in the visual compositions are connected by vectors or eyelines, the participants are depicted as doing something to one another; this image is classified as narrative image. On the other hand, conceptual images contain no vectors (Jewitt \& Oyama, 2001 p. 141). Following this representational meaning, Suen's (2013) study shows that the hotels make use of conceptual images more than narrative images in order to represent the idea of relaxation, hospitality, and elegance of the hotels as well as the scenery of Hong Kong. In regard to the interpersonal function, the most interesting finding is that although the hotels are five-star and luxurious, the web designer usually uses a horizontal angle to create an equal relationship between the hotels and the viewers in an attempt to promote a sense of engagement and warm hospitality.

The last meaning is compositional, which involves the layout and organisation of visual elements in the compositions. The most salient or ideal information is located the top of the page and real or more specific information is located at the bottom of the page (Kress and van Leeuwen, 2006). In relation to the characteristics of web advertising discourse, Suen (2013) suggests that the hotels often adopt a top and bottom approach, which is a typical layout of a homepage to promote hotels. Apart from the use of a top and bottom approach, Suen (2013) does not provide comprehensive descriptions of how navigational links, which are one of the most important features of online advertising, used on the websites. Overall, the study seems to focus more on how the visual elements are represented on the homepages and what kind of effect the hotels aim to convey to the readers through the use of visual images.

In connection to online advertising, Maci (2007) adopts Kress and van Leeuwen's (1996; 2006) framework to explore the visual images on tourism homepages. Compared to Suen's (2013) analysis, this study puts more emphasis on how the visual elements are organised in relation to the digital discourse. Maci (2007) focuses on compositional meaning proposed by Kress and van Leeuwen (1996; 2006), which involves how images are positioned and arranged in a composition. By analysing the placement of visual images on the homepages, their position conveys their relative importance within the homepages. This study shows that apart from the common use of the top and bottom approach to promote their destinations, the tourism homepages also adopt given and new sections. Jewitt and Oyama (2001) and Kress and van Leeuwen (1998) suggest that the left side of the compositions shows something the viewers already know as familiar content. This is referred to as given (Jewitt \& Oyama 2001, p. 148; Kress \& van Leeuwen 1998, p. 189). On the other hand, the 
right side represents the new, something which is not yet known to the viewers, therefore, they should pay attention. However, this reading path from left to right is western oriented. The interpretation may be different when this approach is used in a context where a reading path is right to left. Maci's (2007) analysis seems to correspond to the notion of information value of given/new defined by Kress and van Leeuwen (1998, p. 189). In addition, Maci (2007) found that the main visual images are usually depicted in a central frame, which the navigation links are usually located on the top and on the left of the homepages. This kind of placement seems to be a common layout of webbased advertisements.

The above studies (Maci, 2007; Suen, 2013) are concerned with the investigation of visual resources represented on tourism and hotel homepages. These two studies provide guidelines of how to use Kress and van Leeuwen's framework to analyse visual elements in online discourse. However, their focus is mainly on the homepages. In order to gain a comprehensive idea of how boutique hotels promote themselves through multimodal structures beyond the homepages, I extended the scope of my analysis to three webpages: homepage, accommodation page, and facility page. The details of my study are discussed in Chapter 4: Methodology.

Even though Kress and van Leeuwen's $(1996 ; 2006)$ framework is very influential and is widely adopted as a theoretical platform for visual analysis in various contexts as discussed above, their framework contains certain difficulties when applied to visual image analysis. The most significant challenge of this approach is that the interpretation of the visual resources in relation to interactive meaning of the framework seems to be relatively subjective. This subjectivity issue is evidenced by Suen's (2013) and Rajandran's (2009) studies. Both studies reveal that Kress and van Leeuwen's (2006) framework cannot be applied entirely to all images in this study. For example, Kress and van Leeuwen (2006) explain that an oblique angle causes a sense of detachment; however, in Suen's analysis, the oblique angle images are often used by the hotel in order to create a larger viewpoint of the hotel and capture as many details as possible in one image. Another instance that shows the issue of subjectivity in the application of interactive meaning is drawn from Rajandran's (2009) study of bank brochures. He confirms that the oblique angle and offer images in the brochures convey disengagement between the visual elements and the viewers, which corresponds to the interpretation proposed in the framework of Kress and van Leeuwen (1996; 2006). However, certain aspects of the framework are debatable and can lead to alternative interpretations. These interpretations do not seem to directly reflect the main intention of the bank, whose aim is to use these images to show detachment from their potential customers. These images are used to differentiate between people who have acquired the products and those who have not. The 
message of the images is that the products make customers happy and relaxed and those viewers who do not have the products should be envious and want to have them. This interpretation may be true when applied in a different discourse. These two examples are supportive of the issue of subjectivity of the interpretation of interactive meaning in this approach. The next section discusses the theoretical background of the second approach: systemic functional grammar multimodal discourse analysis.

\subsection{Website design/digital discourse in hotel and tourism context}

This section provides an overview of characteristics of website design and related literature with an emphasis on how promotional discourse exploits web advertising to promote their products or services.

Since the 1990s the Internet has changed marketing practices from traditional print-based hotel brochures to a highly interactive hotel web advertising experience (World Tourism Organization, 1999). The Internet offers inexpensive opportunities for the travel and tourism industry to reach out across the world to promote intangible forms of services to potential guests (Baloglu \& Pekcan, 2006). The introduction of the Internet enables the hotel operators to tangibilise the hotel service by the presentation of multimedia and high definition images (Baloglu \& Pekcan, 2006). Due to the cost-effectiveness of this ideal vehicle for promoting products/services online with no geographic limits, it has become the most powerful marketing medium to date (World Tourism Organization, 1999). Since web advertising is categorised as a promotional genre (Kleindl, 2003), its primary purpose as suggested by Bhatia $(1993 ; 2005)$ and Li $(2005)$ is to inform the readers about products, ideas, or services and persuade them to take action. This is why hotel operators, either big chain hotels or small independent hotels, have widely adopted web-based advertising as a communicative tool to promote themselves and enhance competitiveness (Parets, 2002). My study explores how boutique hotels represent themselves in terms of visual and textual elements as a digital genre. Exploring the special features of web advertising and investigating how web designers organise textual and visual elements to attract the readers' attention provides me with a background and framework to analyse boutique hotel as electronic genres.

Due to the rapid growth of the Internet in online advertising, the terms cybergenres (Shepherd \& Watters, 1999, p. 1) and digital genres (Askehave \& Nielsen, 2005) have emerged. As suggested by Yates and Orikowski (1992) genres change over time in response to the introduction of new technology and the need of discourse community, and this has resulted in the origin of cybergenres. According to Shepherd and Watters (1998), the term cybergenres refers to the 
integration of content, form, and functionality. On the other hand, Askehave \& Nielsen (2005) do not provide a formal definition of digital genres. They only give a simplified synonym "electronic genres" for digital genres. However, their study provides a descriptive explanation of the important properties of web-mediated materials, which enables the readers to understand the concept of digital genres without giving a specific definition.

The difference in the visual representation between online and print- based hotel advertising is due to the medium and the contexts where the promotional texts appear (Janoschka, 2004). These differences include layout, design, images, and interactive multimedia. The ways these two types of advertising are organised and structured creates a different impression on the readers. Print-based tourism advertisements are laid out on a horizontal axis, while in online advertisements, the homepages are laid out on a vertical axis (Singh \& Forminca, 2007). Similarly, Ruffini (2002) points out that the organisation and design of visual as well as textual elements on webpages are different from the organisation and design of print materials. He suggests that the appearance of paper based marketing materials is viewed as whole units, while the layout of webpages is more likely viewed as screens of information. Ruffini (2002) further explains that a homepage layout should be arranged into two main sections: visual and functional. The visual section refers to the top of the webpage, where web designers put the most salient elements in order to make it visible to the viewers. The information represented on the functional zone is less important than the visual zone in that the viewers have to scroll down the page to see the information placed at the bottom. The significance of the visual-functional section seems to correspond to the notion of ideal-real proposed by Kress and van Leeuwen (1996; 2006, p. 219), where the top section of the web pages usually involves eye catching materials to draw the attention of the viewers and entice them to read the rest of the page (Kress and van Leeuwen, 2006, p. 219).

Typically, webpages are organised into five main elements: header, body, right and left margin, and footer. Headers usually consist of the hotel's name and logo, slogan, and menu tabs (navigational links). Body sections contain the main promotional content of the web pages which isusually in forms of textual and visual elements. Right and left margins usually cover menu tabs (navigational links), news and promotions. Footers mainly include copyright, links, and contact information (See Figure 1).

Gilbert and Powell (2001) also suggest that these special features: high-speed of information dissemination, interactivity, reservation channels, multimedia, virtual tour and customerpersonalisation are usually adopted in hotel websites. Similar to Gilbert and Powell (2001), The World Tourism Organization (1999) and Askeshave and Nielsen (2005) also recommend that the 
application of multimedia, virtual tour, and live cams make the page more interesting. Additionally, the nature of online marketing enhances a degree of personalised features and interactive experience of the audience because they take control of the websites in order to choose to see what images and to experience what sounds just by clicking the mouse (Giannopoulos \& Mavragani, 2011; The World Tourism Organization, 1999). This interactive feature also engages the viewers to stay on the page longer because they have an active role to explore the site (World Tourism Organization, 1999). In addition to the attractiveness of visual elements and interactive properties that enhance web exploring experiences of the audience, ease of navigation is also important (Perdue, 2002; Mittal, 1999).

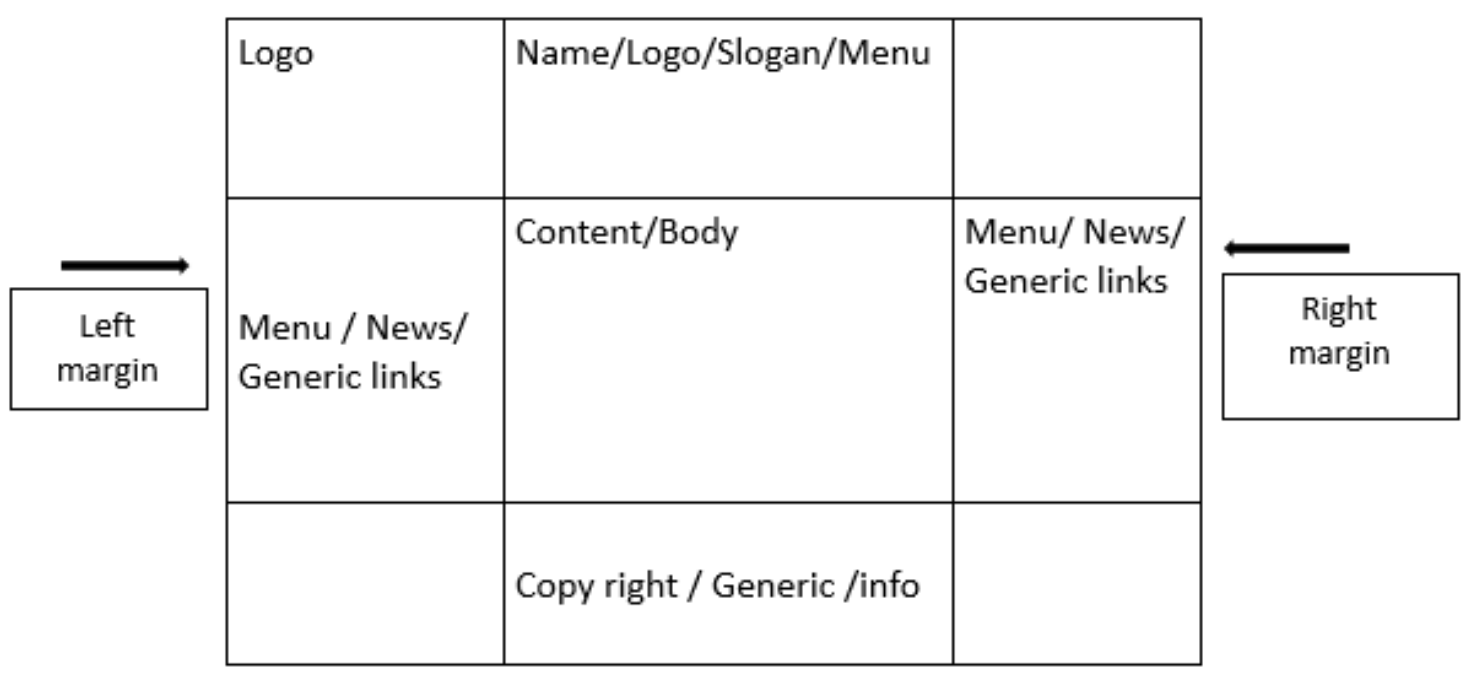

\section{Figure 1: The layout of the webpages}

\subsubsection{Research in website design}

The trend of studies of websites within the promotional contexts of hotel and tourism industry primarily focuses on usability, functionality, performance of websites, and users' perception of online experience. These studies include; the evaluation of the usability of hotel websites in Hong Kong (Yeung \& Law, 2006), the development of evaluation criteria to examine hotel electronic channels of distribution ( $O^{\prime}$ Conner \& Frew, 2004), the investigation and evaluation of the level of website development in New Zealand's regional Tourism organisation (Doolin, Burgess \& Cooper, 2002) the development of a conceptual model for website evaluation in an North American ski resort setting (Perdue, 2002), and the analysis of website design of 4 and 5 star hotels in Turkey (Baloglu \& Pekca, 2006). The findings of these studies reveal that online marketing, website design, and interactive features play an important role to promote their facilities and services in the hospitality and tourism sector. The findings of these studies also correspond with previous research 
(Giannopoulos \& Mavragani, 2011; Gilbert \& Powell, 2001; Singh \& Forminca, 2006) in which the unique features of online advertising usually include a number of different sections of hotel information, email, online reservation, background image and flash animation, corporate identity, consistent navigational links, web links, tourist information, and promotion. However, some studies note that many hotels do not effectively adopt the internet for web-based marketing. For example, Baloglu and Pekcan (2006) investigate the distinctive features (interactivity, navigation, and functionality) exploited in websites of the luxury hotels in Turkey. The findings show that even though these luxury hotels adopt distinctive online characteristics (e.g., online reservations, background images and flash animations, and navigational links/web links) to promote their hotels, they fail to use these features to their full potential.

\subsubsection{Research in digital genres}

Despite the significance of websites in the hotel industry, few research studies have analysed hotel website discourse as a genre. As mentioned, many studies have given a general overview of website content, design, structure, and a measurement to evaluate their effectiveness but very few of them have studied websites as digital genres or cybergenre (Shepherd \& Watters, 1999). In addition, not many studies have provided a guideline focused detailed analysis in terms of the language used, images presented or an ethnographic perspective. This section discusses the studies that do apply genre to websites.

One of the most influential studies that provides a framework for genre analysis in digital discourse is Askehave and Nielsen'(2005) because they incorporate the inherent features of the Internet as a medium to present information. Interestingly, these unique characteristics influence the purpose and the way the web designers promote their linguistic and visual elements online. This is because digital genres incorporate the features of hypertext and interactivity which are the unique properties of digital genre. However, the impact of web-based features on the textuality, proposed by Askehave and Nielsen (2005) is challenged by Janoschka (2004). Janoschka (2004) suggests that even though online advertising tends to use multimedia and interactive features, the differences in terms of linguistic features between online and conventional advertising are subtle.

Askehave and Nielsen (2005) offer a genre analysis model for digital genre analyses. Askehave and Nielsen (2005) also claim that in order to investigate websites, genre analysts should not only pay attention to identify the communicative purposes of the texts but also the navigation links, interactive properties, and multimedia presentations, which are the integral features of webmediated materials. This is because these distinctive features are important to the promotional genre; they are used to engage the readers' attention. Interestingly, Askehave and Nielsen (2005) 
propose that the communicative functions of electronic genres can be conducted in two different modes: reading and navigating modes. They describe their point regarding to the realisation of communicative purpose that the primary purpose of navigation mode is not achieved by structural functional criterion (p.132) but by hyperlinks which is accompanied by textual elements. This genre analysis model proposed by Askehave and Nielsen (2005) is successful as a fundamental tool for genre analysts who are seeking a descriptive framework to investigate genres in web-mediated contexts.

In addition to Askehave and Nielsen (2005), who shed light on electronic genres by providing the genre model as a pioneering framework to analyse web-mediated materials, Santini (2007) also focuses on the genres of web pages. However, her primary focus is not to propose a framework for identifying communicative purposes of genre on webpages. Rather, Santini (2007) describes the vital properties of genre on webpages and explains how linguistic features represented on web pages are distinct from those on other mediums. She suggests that genre on webpages are dynamic, unpredictable, and multifaceted as pointed out in her study (2007 p. 2) that "genres on the web are instantiated in webpages, which are a complex type of document, more composite and unpredictable than paper documents." Askehave and Nielsen (2005) and Santini (2007) convey different purposes when proposing their foregrounds towards genres on digital context. However, they agree that website features (e.g. hyperlinking and interactivity) influence the use of language exploited on website as genre. Nevertheless, the impact of web-based features on textuality, proposed by Askehave and Nielsen (2005) and Santini (2007) is challenged by Janoschka (2004). Janoschka (2004) suggests that even though online advertising tends to use multimedia and interactive features, the differences in term of linguistic features between online and conventional advertising are subtle.

Drawing on the notion of digital genres proposed by Askehave and Nielsen (2005) and Santini (2007), many researchers have applied the concept of genres to investigate the characteristics of online promotional genres. These researchers aim to examine how the members of the promotional genres construct the conventions that are socially recognised by the discourse community in order to achieve their main communicative purposes. More specifically, one of the main purposes of genre studies on the online promotional setting is to investigate the characteristics of rhetorical and visual structures, which are structured by the discourse experts to promote their products and facilities on the websites. These include a study to investigate marketing copy on hotel chain websites (Akkakraisri, 2004), corporate homepages (Askehave \& Nielsen 2005), product reviews on websites (Pollach, 2006) 3-star hotel homepages (Klankana, 2007), serviced apartment homepages (Phongsuwan, 2009) 5-star hotel homepages (Suen, 2013), and electronic products 
online advertisements (Labrador, Ramón, Alaiz-Moretón, \& Sanjurjo-González, 2014). However, thesestudies ignore the influence of web-based characteristics when analysing.

Based on the notion of digital genres, Askehave and Nielsen (2005) conduct genre analysis of corporate homepages. They identify nine moves (e.g. attracting attention, greeting, and indicating content structure), in which the realisation of these nine moves is based on the unique properties of web structures (e.g. hyperlinks and multimedia). However, Askehave and Nielsen's (2005) move analysis seems to be preliminary because they fail to provide a detailed description of whether the moves are optional or obligatory. An explanation suggesting whether the moves are optional or obligatory is vital for English for Specific Purposes (ESP) as the explanation can be used as a guideline for second language learners. , the analysis of moves by Askehave and Nielsen (2005) is not likely to be their main focus. Rather, they aim to propose a framework to investigate genre in web-based materials.

Apart from Askehave and Nielsen (2005), Suen (2013) investigates the rhetorical structures and visual images of five-star hotel homepages in Hong Kong. She applies Bhatia's (2004) genre analysis for examining textual elements and Kress and van Leeuwen's (1996) framework for analysing visual images. Suen (2013) identifies eighteen moves in hotel homepages in which the web-based features are additionally incorporated when analysing moves. However, there are some limitations in her study. Suen's (2013) analysis is quite small scale and limited within homepages, which probably cannot be generalised beyond the context of her study.

Similar to Suen (2013), Klankana (2007) examines the discoursal patterns, linguistic features and pictures in hotel homepages. Klankana (2007) employs Bhatia's (1993) and Askehave and Nielsen's (2005) genre analysis framework. The findings show that the typical hotel homepages contain four main moves, namely, stating the name of the hotel, establishing credentials, introducing the offer, soliciting response. Each move comprises various steps which are the rhetorical elements helping to maintain the communicative purposes of the moves. Each step is realized by content and distinctive linguistic features.

The studies above provide the descriptive background and the characteristics of the online marketing as a genre. Additionally, they remind readers of the possible influences of the Internet towards the structures of online marketing materials when analysing moves. Nevertheless, the application of genre analysis to web-mediated materials has received little attention from genre analysts. These studies mainly focus on the functions and features of homepages. Also, there have been two studies investigating visual elements on the webpages, of which one is relevant to my study because the framework of Kress and van Leeuwen $(1996 ; 2006)$ is similarly adopted. 


\subsection{Conclusion}

The purpose of this chapter is to present an overview of the existing research literature related to genre, boutique hotels, visual analysis, and website design in order to provide the readers with sufficient background in which this study is situated and to identify gaps that I aim to fill. To date, researchers have examined move structures, lexico-grammatical features, and visual images in their analyses of hotel webpages. However, they have largely ignored ethnographic methods and cross-cultural factors. Previous studies were limited to either textual or visual image analysis.

Working on genre studies, Swales' (1990) and Bhatia's (1993) framework have been pioneering and one of the most influential frameworks in English for Specific purposes genre analysis. Later studies on genre have shifted their focus from linguistic levels to examine sociocultural factors. This study draws on text analysis and interviews to examine hotel webpages. Following Kress and van Leeuwen's $(1996 ; 2006)$ framework, this study also focuses on how web designers present their webpages to create persuasive effect and build a relationship with readers. The theoretical and analytical frameworks are also outlined in this chapter, but are not discussed in detail. The next chapter discusses the overall framework applied in this thesis. 


\section{Chapter 3: THEORY}

This chapter presents the theoretical frameworks of my analysis, namely genre theory and the grammar of visual design based on social semiotics theory. As mentioned in chapter 1 , the purpose of my study is to examine boutique hotel webpages as a genre in an attempt to provide language descriptions to novice web designers and small business owners who want to open a boutique hotel. This first part of this chapter discusses the three traditions of genre studies: English for Specific Purposes (ESP), Rhetorical Genre Studies (RGS), and Systemic Functional Linguistics (SFL). It examines their definitions, and key concepts with the aim of developing a theoretical framework for this study. Even though these three schools of genre have been mentioned in chapter 2, I discuss them in more detail in this chapter. Each of these approaches contributes to the framework I use in my analysis and contributes to the insights I am able to offer to professionals in the web design business and to teaching context. The second section discusses the theoretical framework that I used for visual analysis, social semiotic multimodality, which is based on Kress and van Leeuwen's $(1996,2006)$ grammar of visual design.

\subsection{Genre theory}

As explained in the previous chapter, genre has traditionally been a literary concept that focuses on text classification. For the contemporary concept, genre has recently become an influential framework, with an emphasis on social and contextual aspects in the analysis of structural elements and their functions in non-literary texts. The most influential definitions of genre are from three different schools of genre - English for Specific Purposes (ESP), Rhetorical Genre Studies (RGS), and Systemic Functional Linguistics (SFL). The differences in emphasis and analytical approach between each genre tradition have resulted in the different ways that those genres are articulated, analysed, and used.

\subsubsection{English for Specific Purposes (ESP)}

The first tradition of genre theory that I discuss here is English for Specific Purposes (ESP). My analysis of boutique hotel webpages is primarily situated in this approach because of the approach's applicability to professional writing and its pedagogical implications. The ESP genre approach is also the most applicable framework and forms a basis of my analysis because it focuses on both the macro (move structures) and micro (linguistic features) structure of a text. Before discussing the ESP genre analysis framework proposed by Swales $(1990)$ and Bhatia $(1993 ; 2004)$ on 
which I based my analysis of online advertising materials, it is essential to explain how genre is defined by Swales (1990) and Bhatia (1993; 2004). Swales (1990, p. 59) defines genre as a class of communicative events employed by specific discourse communities who share a set of communicative purposes. The communicative event involves the discourse and its context as well as participants. Communicative purposes refer to speakers' or writers' intention. Swales (1990, p. 59) points out that the communicative purposes provide the rationale for the genre and serve as a primary criterion to identify genre and also to distinguish one genre from another because they essentially determine the choices of content and structures as well as linguistic features. For example, the purpose of the hotel web advertising genre is to promote facilities and services. To achieve this purpose, web designers use Move: describing rooms to describe and advertise the accommodation and facilities in the room.

Early works in ESP primarily focused on the quantitative aspect of language, with an attempt to identify the frequency of occurrence of certain lexico-grammatical features in a particular discourse in order to provide language descriptions. These formal features of a genre are important and should not be neglected because they are used to describe the characteristics of genres (Vergaro, 2004). However, in recent years, ESP genre theorists have viewed genre as more dynamic and complex, as suggested by Askehave and Swales (2001), Bhatia (2004), and Johns et al. (2006). Askehave and Swales (2001) explain that due to the increasing number of genre studies and the increasing complexity of the conceptualisation of genres, the idea of communicative purposes has also become more complex but still remains a key concept in several genre-based studies. Askehave and Swales (2001) suggest that since some texts have multiple purposes, it is important to investigate the contexts in which the texts are used when analysing genres. The idea of the complexity of genre is evidenced in Samraj's (2004) study, where she found that in academic genres for example, research articles produced from different courses within the same discipline (environmental science) show internal diversity. Nevertheless, these courses still share some core similarities showing that they are members of the same genre. The findings show that each genre has its own constraints that determine the value and characteristics of genres.

Owing to the fluidity of genre discussed above, many genre theorists aim to investigate the contextual factors that influence variations. In line with Miller's (1984) social action concept, ESP's view of genre has shifted from focusing on the textual features of language to a deeper investigation of communicative purposes and effects. Following Swales' definition of genre, Bhatia (1993) puts forward a critical framework that accounts for textual and ethnographic aspects of genre analysis. Bhatia (1993) argues for integrating contextual factors into genre studies. He bridges the gap between ESP and RGS genre approaches by combining ethnographic approaches into genre analysis 
of professional discourses in order to provide detailed descriptions of the contexts surrounding genres. In his book World of written discourse: A genre-based view (2004), he advances the notion of genre that contextual factors are important to the contribution of genre construction, understanding, and interpretation. In summary, Bhatia (2004) defines genre as follows:

Genre refers to language use in a conventionalised communicative setting in order to give expression to a specific set of communicative goals of a disciplinary or social institution, which give rise to stable structural forms by imposing constraints on the use of lexico-grammatical as well as discoursal resources (p. 23).

Bhatia's definition of genre is relevant to my cross-cultural study, in which I aim to investigate contextual features that shape the characteristics of the hotel web genre in two different settings. Bhatia (2004) takes up Swales' (1990) argument for the need to go beyond structural and linguistic investigations of texts in order to understand social and contextual features of genres. Similarly, Badger (2003) proposes that as genre and communicative purposes are interrelated, the knowledge of contexts in which a genre is situated contributes to the understanding of how a genre is constructed. As a result, in addition to linguistic evidence, the knowledge of socio-cultural aspects where a genre is located is an important key for discourse analysts to understand genre behavior. In an attempt to examine the social and cultural contexts of a particular genre, several studies have integrated ethnographic approaches from Rhetorical Genre Studies to investigate genres and their communicative purposes (Flowerdew \& Wan, 2010; Hyland 2002; Hyland 2004; Hyon 2002; Paltridge, 2002; Suen, 2013; Swales \& Lindemann 2002).

As proposed by Swales (1990) and Bhatia (1993), genres are purposeful activities, in which the shared communicative purposes are the key defining features for classifying genres. Since most of Swales' work has focused on the introductions in research articles, Bhatia's $(1993,2004)$ perspective of genre is the most relevant to my genre study. I based the analytical framework for my genre study on Bhatia's $(1993 ; 2004)$ genre perspective. In my study, boutique hotel webpages emerge as purposeful activities that aim to, for example, inform about the existing products and services and persuade the potential readers to buy or use products and services. In order to identify the functions and rhetorical structures of hotel online advertising materials and go beyond the textual analysis, I used Bhatia's (1993; 2004) genre approach because this approach informs my study in the most important way. His framework contributes to the investigation of genre by the inclusion of socio-cultural aspects in order to gain thicker description of genres. In the following section, I will discuss the genre analysis approach that has been constructed by Swales (1990) and further developed by Bhatia (1993; 2004) in an attempt to provide detailed description of genres. 
In the area of ESP, the underlying principle is to identify the communicative purposes and conventionalities of genre in its context in order to provide learners with the appropriate language resource that they require in their studies or professional lives (Swales, 1990; Bhatia, 1993). The main analytical tool is move analysis, which is used to identify communicative purposes. It is applied to identify moves or functional components or semantic units (Upton \& Conner, 2001, p. 5) and to identify steps of a genre by classifying textual structures according to their communicative functions (Swales, 1990). Swales (1990) and Holmes (1997) put emphasis on the importance of communicative purposes because they explain that communicative purposes influence the content and choices of language that the writers use to structure their texts. In line with Swales (1990), Henry and Roseberry (1998) suggest that moves are rhetorical organization of a text (p. 147). In their genre study of application letters, Henry and Roseberry (2001) define a move as a functional unit, explaining that a move can be considered "as part of a text, written, or spoken, which achieves a particular purpose within the text" (p. 154).

Similarly, according to Bhatia (1993), a move refers to a segment of a text that fulfils a specific communicative function. Within a particular genre, each move reflects a specific communicative purpose, which contributes to the overall purpose of the genre. Consistent with previous studies, Flowerdew and Dudley-Evans (2002) describe a move as a schematic structure or staging (p. 469). In their study of editorial letters, Flowerdew and Dudley-Evans (2002) explain that genres are structured based on the communicative purposes that they fulfil and, within genres, texts are subdivided into moves or stages, which contribute to the overall functions of the genres. Another definition of moves appears in Biber, Connor, Upton, and Kanoksilapatham (2007), who define moves as "a section of a text that performs a specific communicative function" (p. 23). They also suggest that "each move not only has its own purpose but also contributes to the overall communicative purposes of the genre" $(2007$, p. 23).

Even though there are various definitions of moves proposed by different genre theorists, they share similarities. It can be concluded that moves are rhetorical units which are characterised by their functions, and these functions contribute to the overall communicative purpose of the genre. However, the identification of moves and steps does not have standardised rules or explicit formulae. Furthermore, Swales (1990) is not able to delineate the distinction between a move and a step; resulting in criticism of Swales' analytical framework. According to Lewin, Fine, and Young (2001), Swales' move analysis model fails to offer systematic criteria for the identification of moves and steps and it leads to problematic application of move analysis approach in other texts. Genre analysts generally rely on linguistic evidence, comprehension of the texts and understanding of the 
discourse community when analysing moves (Biber et al., 2007; Dudley-Evans, 1994; Kanoksilapatham, 2003).

Since genres change over time, Chiavetta (2006) and Samraj (2004) suggest moves that are used to achieve communicative purposes where genres are situated are also dynamic. The dynamic features of moves described by Biber et al. (2007) and Swales (2004) include: 1) moves can be constructed by various linguistic structures, 2) moves have different sequential orders, 3) moves have a recurring nature, and 4) the frequency of occurrence of moves varies.

Moves that occur more often than others are referred to as conventional (Biber et al., 2007) or obligatory (Bhatia, 1993; Swales, 1990). On the other hand, moves that appear less frequently, are defined as optional (Biber et al., 2007; Swales, 1990) or subsidiary (Mulken \& van der Meer, 2005). Determining the frequency of occurrence of moves, whether they are conventional or optional, is problematic due to a lack of explicit criteria and guidelines. This may be because originally, Swales (1990) used move analysis to identify textual structures of the Introduction section of research articles. According to Swales (1990), the introduction sections are generally less diversified; as a result, their move structures are relatively straightforward. However, in his later work, Swales (2004) revises his move analysis framework to be more responsive to the fluidity of genres. In his modified genre framework, he reconsiders that genres are not static but dynamic. He also focuses on the importance of the cyclicity and the frequency of moves (obligatory and optional). Based on Hasan (1989), obligatory moves are necessary to fulfil the communicative purpose of the genre, while optional moves are rhetorical devices that the experts in the community can select to enhance the effectiveness of the genres.

Since the criteria to identify the typicality of moves are not definite, previous genre studies have established their own principles to justify whether moves are obligatory or optional. For example, Kanoksilaphatham (2005; 2007a; 2007b) suggests that obligatory moves are moves that occur more than $60 \%$ of the time. On the other hand, moves that appear less than $60 \%$ are considered as optional. Following Kanoksilaphatham (2005), Tisapramotkul (2007) adopts the cut-off frequency of $60 \%$ to analyse and compare move structures in English recruitment advertisements between Thai and British newspapers. According to Rasameenin's (2006) move analysis of MA thesis discussion sections, moves are categorised into three types based on their frequency: Obligatory (100\%), Conventional (66\%-99\%), and Optional moves (less than 66\%). Drawing on Rasameenin's study, Salmani Nodoushan (2012) and Salmani Nodoushan and Montazeran (2012) applied this cut off frequency to identify moves in discussion sections in MA English theses written by Iranian EFL students and book reviews from applied linguistics journals respectively. 
In contrast, relevant to the notion of typicality of moves, in her genre analysis of tourist leaflets distributed in America, Boonchayaanant (2003) proposes the cut-off frequency of obligatory moves at more than $50 \%$ and optional at less than $50 \%$. This criterion has been adopted by many studies of hotel and tourism promotional materials. These studies include an investigation of travel articles in Tropical Magazines (Chanpetch, 2011); a genre analysis of tourist attraction leaflets produced and distributed in Thailand in 2004 (Mongkholjuck, 2008); and tourist leaflets of the Tourism Authority of Thailand (TAT) (Sinraksa, 2009). Another cut-off frequency is proposed by van Mulken and van der Meer (2005). They state that moves that occur in $75 \%$ or more of texts are obligatory, but optional or subsidiary when they occur in less than $75 \%$ of texts (p. 100). My move analysis adopts $75 \%$ cut-off frequency based on van Mulken and van der Meer's (2005) study. This is because the $75 \%$ cut-off frequency covers the main communicative purposes of my dataset. The discussion above shows that the notion of typicality of moves is arbitrary and there is no agreed cut-off frequency to cope with the diversity of moves. Even though some studies offer models to justify and classify the frequency of each move, they do not provide an explanation as to how they derive the frequency.

Another important concept, which is related to the move analysis framework is steps. According to Swales (1990) each move consists of a number of steps realised by particular linguistic features to serve its specific function. Many scholars attempt to define step. For example, steps refer to strategy (Bhatia, 1993; Henry \& Roseberry, 2001) or rhetorical options which discourse members can select to realise moves. Similar to Bhatia (1993) and Henry and Roseberry (2001), Kanoksilapatham (2007a, p. 173) refers to steps as subunits that are used to fulfil the purpose of moves. In this sense, a move refers to a rhetorical unit that is used to achieve the overall communicative purpose of a particular genre, while steps are rhetorical options that are used to realise a particular move. A step is a smaller unit than a move, located on a subordinate level to it. The writers are able to select a step or steps to realise a move. In addition, some moves may be realised through a variety of steps or strategies (Bonyadi, 2012). In order to have a clear understanding of how moves and steps are connected and contribute to the overall communicative purpose of a particular genre, I provide the following example from Kanoksilapatham (2007b). 
Table 5: an example of a move and steps in scientific research articles

\begin{tabular}{|l|}
\hline Move 3: Introducing the present study \\
\hline Step 1: Stating objectives \\
\hline Step 2: Detailing procedures \\
\hline Step 3: Justifying decision made \\
\hline Step 4: Presenting findings \\
\hline Step 5: Stating implication \\
\hline
\end{tabular}

Table 5 shows how the main communicative purpose of Move 3 (Introducing the present study) in Kanoksilapatham's work (2007) is functionally connected to the five steps. That is to say, in order to fulfil the functional purpose of Move 3, there are five rhetorical options available for the researchers to choose.

In the move analysis framework, apart from move and step identification, many genre scholars also pay attention to distinctive linguistic features that are shaped by the communicative purposes of the genre (Bhatia, 1993; Bonyadi, 2012; Dudley-Evans, 1994; Henry \& Roseberry, 1998; Henry \& Roseberry, 2001). Dudley-Evans (1994) suggests genre analysis also involves the investigation of lexical frequency and the use of tense. This investigation contributes to an identification of the conventions of a genre. Consistent with Dudley-Evans (1994), Bhatia (1993) also points out that an investigation of lexico-grammatical features is essential in genre studies because the findings at this linguistic level can reveal specific linguistic features that are associated with certain moves and steps. This idea is supported by Henry and Roseberry's (2001) and Bonyadi's (2012) suggestion that there are certain lexico-grammatical structures that are frequently associated with specific moves and steps. For example, Bonyadi (2012) illustrates that the Move: orienting the reader in newspaper editorials exhibits two different linguistic realisations; a declarative sentence and a rhetorical question. In short, the key components of genre analysis embody an identification of communicative purposes, move and step patterns, and lexico-grammatical features that are associated with the moves and steps. The next section discusses Rhetorical Genre studies that I based my study on for the investigation of insiders' perspective.

\subsubsection{Rhetorical Genre Studies (RGS)}

The second tradition of genre is Rhetorical Genre studies (RGS). I rely on this school mainly because of the significance of an ethnographic approach, which is the primary value of this school. Rhetorical Genre theorists view genres as a social process because genres are used to fulfil actions in 
different social settings. This notion is based on Miller (1984) who focuses on rhetorical contexts in which genres are utilised rather than the linguistic features of the genres. Miller (1984) proposes that genre is "typified actions based in recurrent situations" (1984, p. 151). Therefore, the primary focus of RGS is on a social action, which dwells in the situational contexts and intention of the speakers. The interests, purposes and shared assumptions of discourse communities are the most important elements in this genre tradition. Miller's (1984) concept of genre constitutes a rationale for genre analysts who are interested in exploring cultural patterns and practices and those who would like to understand and participate in particular social actions. As I explained earlier, apart from exploring the regularities of the rhetorical structures of boutique hotel webpages, I also drew on Miller's social actions in order to gain insight into the conventions of web designers and boutique hotel operators' practice in developing hotel webpages. Berkenkotter and Huckin (1995, p. 7) maintain that the construction of genre is "our participation in the communicative activities of daily and professional life." Their comment implies that the Rhetorical Genre theorists pay attention to how discourse experts implement genre knowledge to perform their actions in the community. In addition, these two influential Rhetorical Genre theorists state that genres are dynamic and change over time; they are shaped by the situational contexts and intentions of discourse members. In order to understand the intention of a particular discourse community, an investigation into how discourse members apply genre knowledge to participate in the community is needed. This answers the question of why ethnographic methods are major research tools, which are used to explore the practices of the members of a discourse community.

Discourse analysis research in the Rhetorical Genre tradition has relied on ethnographic methods (interviews and participant observation) in order to investigate how genres are derived from and interact within their context. By doing so, scholars pay attention to contextual aspects and participants who construct the genres rather than mainly focusing on textual analysis (Hyland, 2001). Hence, this area of genre theory is ethnographic rather than linguistic. Researchers have used ethnographic methods to provide a comprehensive explanation of how situational contexts shape genres. In Rhetorical Genres studies, the main focus is on the exploration of sociocultural aspects of genre such as culture, gender, and social class. Drawing on RGS, my study applied an ethnographic method (interviews) in order to discover the reasons behind the similarities and differences between boutique hotels webpages in Thailand and in New Zealand. ESP and RGS are considered as complementary genre approaches to investigate the rhetorical structures and to understand the practices behind the developing procedures of boutique hotel webpages. The following section focuses on Systemic Functional Linguistics (SFL), which informs my study in terms of analytical application of visual analysis. 


\subsubsection{Systemic Functional Linguistics (SFL)}

In Halliday's (1985; 1994) Systemic Functional Linguistics, the underlying focus is on the social function of language use as it primarily pays attention to the relationship between language and its functions in social contexts. Halliday (1978) describes language use or register as "the configuration of semantic patterns that are typically drawn upon under the specific conditions, along with the words and structures that are used in the realisation of these meanings" (p. 23). Register is used to define characteristics of texts, which are governed by contexts. The key aspects of the contexts can be defined as: field (what is happening), tenor (the relationship between participants), and mode (the medium of communication) (Bawashi \& Reiff, 2010, p. 30; Figueiredo, 2010, p. 124).

To further the notion of register pioneered by Halliday (1978), Martin (2001) takes the vital step of constructing the relationship between genre and register, where genre is viewed as context of culture, while register provides the context of situation (Martin, 1997). That is to say, register conforms to the situational contexts, while genre conforms to the cultural contexts (Figueiredo, 2010). Martin's (1997) proposition of the interaction between genre and register enhances the understanding of how social purposes are connected to textual structures and how these connections predict certain linguistic resources within register (Feez, 2002). In Martin's view (2001), "a genre is a staged, goal-oriented, purposeful activity in which speakers engage as members of our culture" (p. 155). Genres are goal-oriented because they are used as means to achieve the goals of community; and they are staged because they often exploit more than one step to achieve the goals (Martin, Christie \& Rothery, 1987). In this sense, Martin's genre analysis approach mainly focuses on the stage and goal oriented aspects of genre. Within this view, Bateman (2008, p. 85) advanced the idea that genre is "staged sequences of purposive actions", where each stage contains a particular register. Genres are functional since their structure serves social purposes and are also observable through specific register or style of language use. In this sense, genre and register heavily rely on each other in order to achieve community goals; genre analysis should pay attention to both the genre and its register because they are connected and complement each other. That is to say, it is important in SFL to investigate how social purposes are connected to textual patterns and how these social purposes are socially and linguistically realised within the register (Fakhruddin \& Hassan, 2015).

With regard to genre studies, SFL has made two contributions. The first is Halliday's (1978) notion of register, where a particular set of semantic/lexico-grammatical patterns correspond to its situation (Bawashi \& Reiff, 2010, p. 31). The second is the interaction between genre and register proposed by Martin (2001). These two notions of genre have provided students with appropriate linguistic resources relevant to their requirements and social contexts (Chen, 2008; Christie, 1987; 
Lock, 1996). Examples are Cheng (2007): teaching genre to improve writing; Parkinson \& Adendorff (2004): using popular science in teaching scientific literacy; Parkinson \& Adendorff (2005): using science books to enhance textbook literacy in children; and Macken-Horarik (2002): teaching genre in classroom.

Apart from the contribution of genres toward pedagogy, the SFL genre analysis approach has been extended to multimodal analysis. This extension was influenced by the fact that the genres are observable and predictable. Later on, this notion was applied to investigations of multimodal elements (Bateman, 2008). Kress and van Leeuwen's $(1996 ; 2007)$ multimodal approach is one of the most influential frameworks, which has been widely adopted by Almeida, (2009): advertisement; Hiippala, (2012): tourist brochures; Rajandran, (2009): bank brochures; and Suen, (2013): hotel websites. My study has also drawn on Kress and van Leeuwen's (1996; 2006) multimodal framework to examine images that are used on boutique hotel webpages. A detailed discussion of Kress and van Leeuwen's framework will be provided in section 3.3.2.

This section discusses the similarities and differences between SFL, and the other two traditions - RGS and ESP. An aspect common to both SFL and RGS is integration of social factors in order to investigate genres. However, the fundamental purposes of these two traditions are different. RGS tries to provide a thick description of a genre, while SFL focuses on textual features in relation to contexts.

On the other hand, ESP and SFL hold that language is connected to social context and function. Both genre traditions similarly underline the importance of pedagogical implications. However, according to Bawarshi and Reliff (2010), ESP and SFL differ in interpretation and understanding of contexts. SFL theorists define genre in terms of their social purpose, which is socially recognisable. On the other hand, in ESP, genres are realised by their communicative purposes, which are determined by speakers' or writers' intention and it is not socially recognisable.

Consequently, Bawarshi and Reliff (2010) point out that since SFL pays attention to context of culture, their focus is on what Bawashi and Reiff call pre-genres or abstract form of genre such as narrative in order to describe contexts at a macro level. In contrast, ESP genre approach is located in more defined contexts, where communicative purposes are more specific and described in greater detail. The link between defining genre and discourse community enables ESP genre analysts to deal with context and communicative purposes (p. 44). In addition, ESP pays attention more to the discourse community where a thicker description of genre can be investigated compared to SFL. Due to the difference in a genre's rationale between ESP and SFL, I based my study on the ESP genre approach because the main focus of this tradition is to serve ESP pedagogical and professional implications by offering the linguistic resources of a particular genre. The discourse structures are 
identified in terms of moves and functions. Specifically, this ESP rationale frames my research direction as the context of the genre (hotel webpages) is specified and attributable, which enables me to identify communicative functions of boutique hotel webpages and practices behind webpage development.

Since genres are intrinsically complex, a single approach from each school may not allow discourse analysts to understand the comprehensive configurations of genres. This is because each genre tradition has its own focus. As a result, as discussed above, I used a combination of the approaches from the three schools to analyse boutique hotel webpages as a genre.

In conclusion, this section presents different definitions of genre and key concepts within genre-based approaches in the three traditions of genre studies, with the aim of establishing my analytical framework for this study. Within these three schools of genre, various analytical perspectives and approaches are complementarily drawn on to inform my framework for the genre analysis of boutique hotel webpages. The next section deals with Multimodal analysis approach that is further developped from SFL by Kress and van Leeuwen (2006).

\subsection{Multimodal Discourse Analysis (MDA)}

Visual images in online advertising play a crucial role in stimulating desire in the target audience. They attract the readers' attention, promote product features and arouse emotion (O'Guinn, Allen, \& Semenik, 1998). I consider hotel websites as an example of multimodal discourse due to the combination of textual features, visual design, and functionality. However, the functionality is not my research focus. In addition to the analysis of textual elements, the present study also examines how web designers present visual images in hotel webpages in terms of the position of the images in the visual space and the relationship created between images and readers. This section focuses on social semiotic multimodal approach in the multimodal domain, with a particular emphasis on Kress and van Leeuwen's (1996 \& 2006) framework, which forms the basis of my visual image analysis.

\subsubsection{Multimodal approaches}

Halliday's (1985) and Halliday and Matthiessen's (2004) functional grammar provides the foundation for multimodal research. As mentioned ealier in this chapter, the main focus of Systemic Functional Linguistics (SFL) is on the relationship between texts and their contexts in which texts are constructed by three metafunctions: the representational, interactional and compositional metafunctions. According to SFL, all acts are "communicative artefacts", which are constructed by the socio-cultural context and simultaneously characterised by three types of meaning: ideational, 
interpersonal, and textual (Bateman, 2008; Djonov, 2008). Bateman (2008) asserts that as the main focus of SFL is on meaning, this leads to the extension of the SFL framework to multimodal analysis approach. This is because Multimodal SFL theorists consider images as another act of communication that is produced by the three metafunctions. Drawing on Halliday's theory, three major approaches to multimodality evolved from three different theoretical backgrounds: social semiotic multimodal analysis, systemic functional multimodal discourse analysis, and multimodal interactional analysis (O'Halloran, 2013; Jewitt, 2009b). Each approach enables researchers to explore different kinds of questions and select different frameworks that suit their data. My study drew on social semiotic multimodal analysis which I will explain in the next section.

\subsubsection{Social semiotic multimodality}

Social semiotics aims to describe and understand how professional communicators produce and convey meaning in specific social settings (Kress \& van Leeuwen, 1996). Social semiotics stems from Halliday's theory, in which the main focus is to explore the relationship between the language and the social context. Social semiotics provides a useful framework for understanding how hotel online advertising discourse is constructed using a variety of principles as proposed by Lemke (1990), who said that social semiotics draws on a combination of several methods to investigate social meaning and social action. Drawing on social semiotic theory, Kress and van Leeuwen (1996; 2006) developed the pioneering and comprehensive multimodal approach to visual element analysis, which they called "The Grammar of Visual Design". Since hotel websites contain both textual and image features which are important to create a persuasive effect for the audience, I am interested in exploring how this effect is created by the visual compositions. My study relies heavily on Kress and van Leeuwen (2006) in order to investigate the semiotic features of web-based advertising. The following section presents Kress and van Leeuwen's framework that informs my present study.

Kress and Van Leeuwen's (2006) theoretical basis for understanding visual images focuses on three metafunctions: Representational meaning, Interactive meaning, and Compositional meaning.

\section{Representational meaning}

Representational meaning refers to visual images, which depict participants that may be people, animals, places or objects. These participants may perform actions and/or interact with each other or the viewers to create meaning in the visual images (Jewitt \& Oyama, 2001 p. 141; Unsworth 2001 , p. 73). As suggested by Kress and van Leeuwen (2006, p. 49-50), participants in visual compositions can be illustrated based on their functional roles in the context of the image. There are two kinds of participants. Interactive participants are those who view images. On the other hand, 
Represented participants are the characters that construct the subject matter of communication in images. They could be people, places and things (p. 48) that are performing a particular role or being depicted in visual structures.

Based on the representational meaning proposed by Kress and van Leeuwen (2006), there are two kinds of images: Narrative and Conceptual patterns. Narrative pictures relate participants in terms of doings and happenings. These kind of images are realised by a vector emanating from participants who are depicted as performing roles or doing something to or for each other (p. 59). The vector associates with a dynamic, doing or happening kind of relation (Jewit \& Oyama, 2001 p. 141).To realise whether the images are considered as narrative or conceptual patterns, the representation of vectorial relations between the participants is the main indicator. When participants or characters in the visual compositions are connected by vectors or eyelines, the participants are depicted as doing something to one another; this image is classified as narrative representation. On the other hand, conceptual images contain no vectors (Jewitt \& Oyama, 2001, p. 141) and do not involve action on the part of represented participants. Narrative pictures visually depict unfolding actions and events, processes of change, and transitory spatial arrangements (Kress \& van Leeuwen, 2006, p. 79), as will be shown in Figure 4 below.

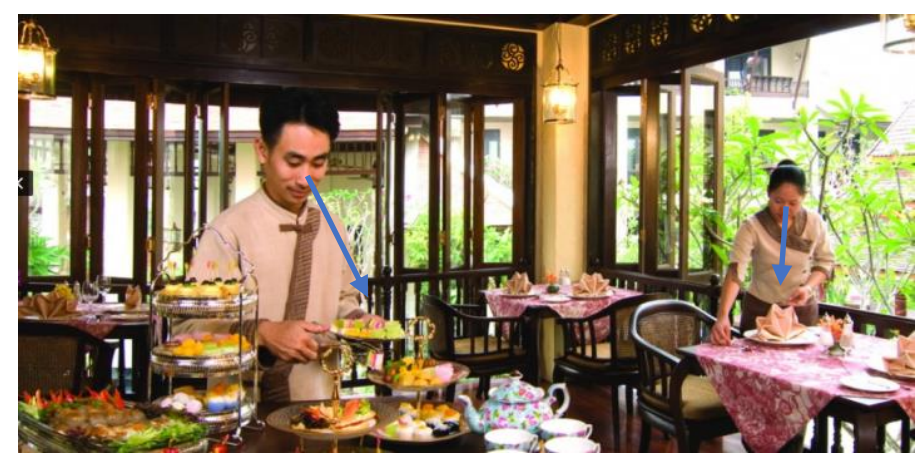

Figure 2 Narrative image

Source: http://puripunn.com/dinning-spa/

Figure 2 shows a Narrative image of two hotel staff in light brown Thai traditional style hotel uniforms, who are preparing food and setting the table. There are two interactions in this image. The first interaction is between the male staff member and the food that he is preparing. Here the vector emanates from the arms and the eyes of the male staff member. The second interaction is between the female staff member and the table that she is setting. The vector is formed by the downward diagonal vector of the woman's arm and her eye line. Both actions are represented by the vectors emanating from the hotel staff member who is the actor or the doer of the action. Figure 2 does not show the reciprocity between the two hotel staff and the food that they are preparing. Rather, it 
represents (the two hotel staff) who is playing the active role of doing and what (the food) the passive roles of being acted upon.

Conceptual images refer to images which do not contain vectors and wherein participants are described or depicted in a more general static manner (Jewitt \& Oyama, 2001, p. 143; Kress \&van Leeuwen, 2006, p. 79). Figure 3 depicts a conceptual image showing a hotel exterior and landscape.

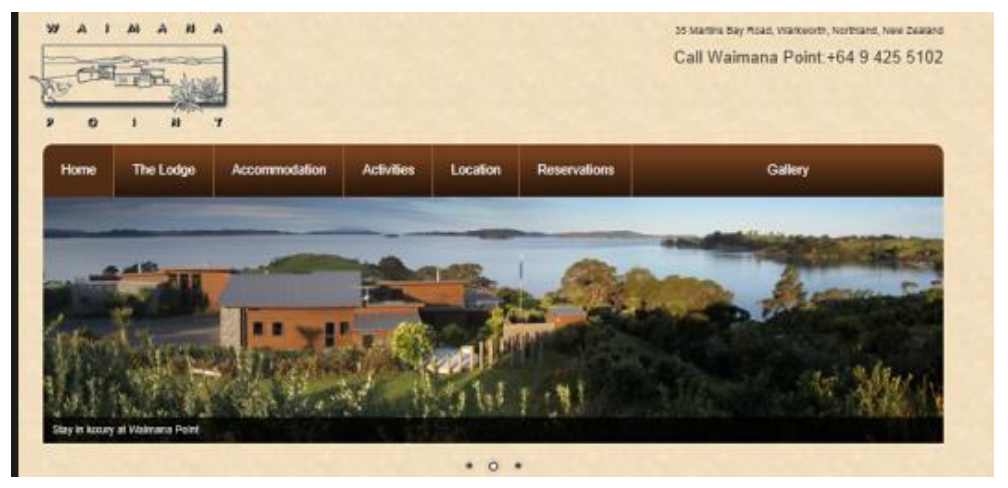

\section{Figure 3: Conceptual image}

Source: http://www.waimanapoint.co.nz/

\section{Interactive meaning}

Interactive meaning refers to imaginary relations between images and viewers of images that occur through their interaction (Jewitt \&Oyama, 2001, p. 145). Three concepts: gaze, size of frame and perspective can be used to analyse the degree of Interactive meaning in the visual images. Gaze refers to whether human represented participants have eye contact with viewers in order to establish an imaginary relationship with those viewers who are invited to engage interpersonally with the represented participant. In this manner, the type of gaze described is referred to as a demand (Jewitt \& Oyama, 2001 p. 145; Unsworth, 2001 p. 95), as seen in Figure 4.

Figure 4 shows a demand image of a woman smiling and having direct eye contact with the viewers. It seems that she is inviting the viewer to come to have a massage with her. On the other hand, when the represented participants do not have eye contact with the viewers, there is no imaginary interpersonal relationship between the viewer and the represented participants. The image is considered as an offer (Kress \& van Leeuwen, 2006 p. 119). An example of this is demonstrated in Figure 5. 


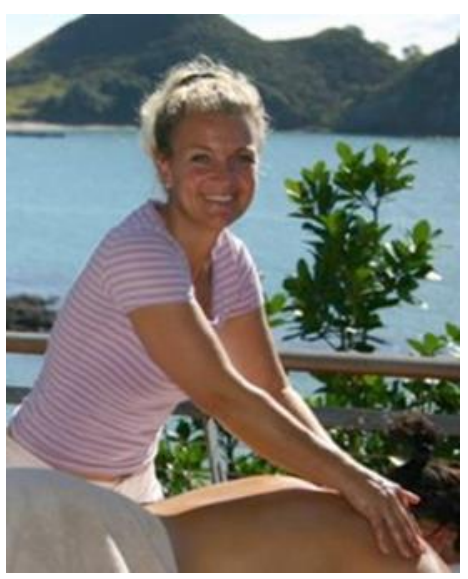

Figure 4: Demand image

Source: http://www.cavallibeachhouse.com/therapeutic-massage/

In Figure 5, the participants are not looking at the viewers but they are depicted as involved in a wedding ceremony. This image is an offer because there is no direct gaze from the represented participants and no imaginary relationship established here. Instead, it seems to inform the viewers that the hotel can organise wedding ceremonies for the guests.

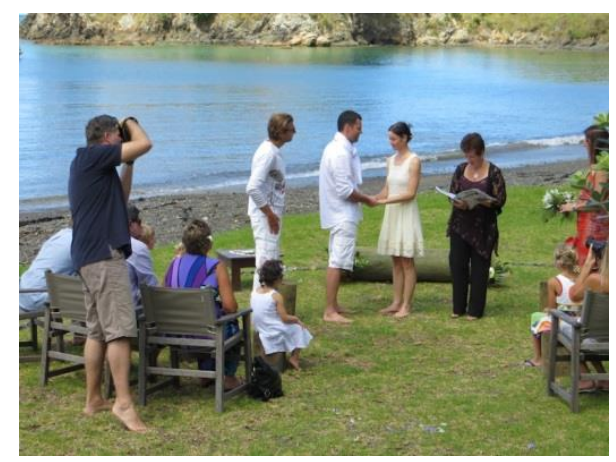

Figure 5: Offer image

Source: http://www.cavallibeachhouse.com/weddings-honeymoons/

The next concept is size of frame. This deals with how close the participants are depicted in relation to the viewers. The varying degree of distance between them creates different levels of closeness. According to Kress and van Leeuwen (2006 p. 124), size of frame is defined in relation to the choice between close-up, medium shot and long shot. This system indicates the distance between the represented participants and the viewers. When the image presents people, the close shot offers an intimate relationship. In addition, Jewitt and Oyama (2001 p. 145) suggested that the close-up shot enables the viewers to observe the individuality and personality of the characters depicted in images (see Figure 6). The medium shot is more objective and social (see Figure 7). The 
long shot makes the viewer more detached, and is therefore ideally used with strangers (see Figure 8). Unsworth (2001) notes that size of frame can also be adopted to investigate the visual structures of non-human participants e.g. buildings and landscape. Hence, this notion is helpful to my dataset since boutique hotel webpages frequently contain images of accommodation, restaurants, and landscapes depicted from different sizes of frame.

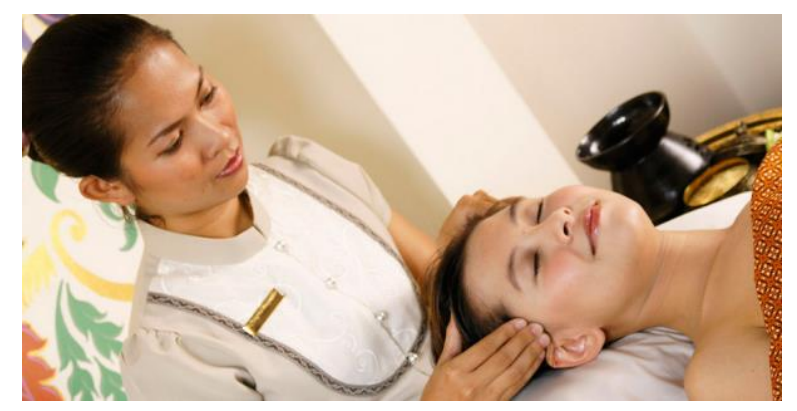

Figure 6: Close-up shot image

Source: http://www.pingnakara.com/spa

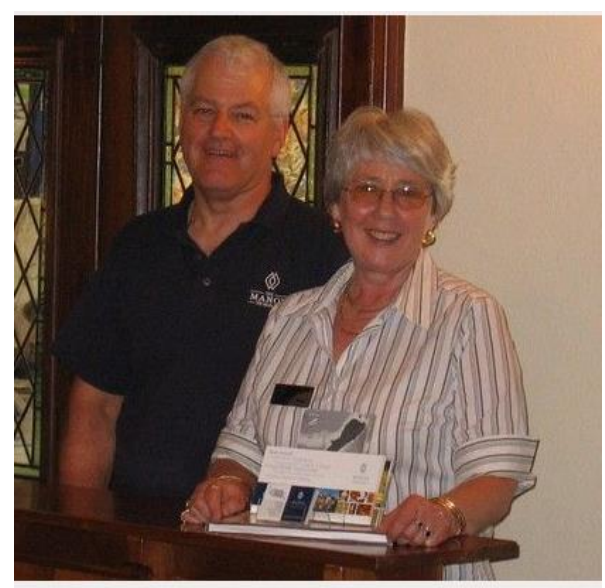

Figure 7: Medium shot image

Source: http://www.elizas.co.nz/ 


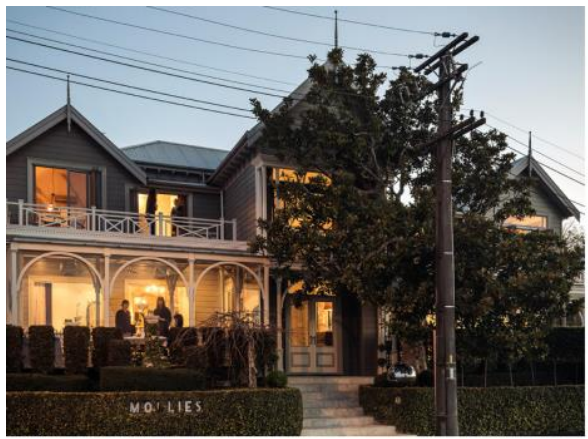

Figure 8: Long shot image

Source: http://www.mollies.co.nz/

The last concept of Interactive meaning is perspective. As far as perspective is concerned, three main angles can be applied: frontal, oblique, and vertical. Unsworth (2001) suggests that the frontal angle indicates involvement between the viewer and represented participants. It shows engagement, as if there is a connection between the viewer and the represented participants ( $p$. 98). Figure 9 depicts a frontal view of a married couple who are walking down the steps. From the frontal angle, the viewers can see the smiling facial expression of the participants. An interpretation with respect to perspective would posit that this couple is happy because they are just married, and they are inviting the viewers to witness their important day.

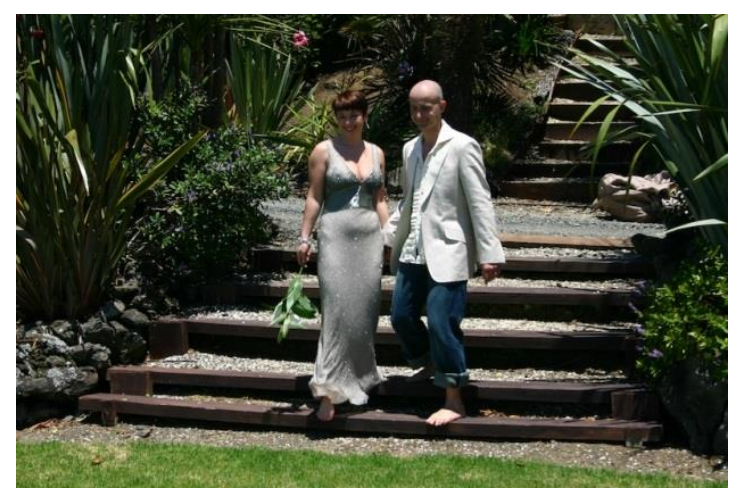

Figure 9: Frontal angle

Source: http://www.cavallibeachhouse.com/weddings-honeymoons/\#jp-carousel-768

In Kress and van Leeuwen's (2006) framework, oblique angle is associated with a sense of detachment. The viewers are not invited to become a part of the image; they only see how the images are represented. Figure 10 portrays an oblique image where the represented participants are 
depicted from the side. It seems that they are discussing a wedding ceremony plan, and as a result, they are not interacting with the viewers.

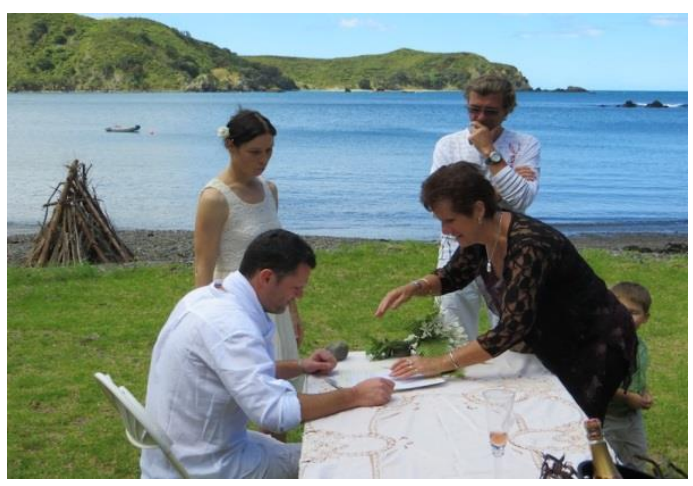

\section{Figure 10: Oblique angle}

Source: http://www.cavallibeachhouse.com/weddings-honeymoons/\#jp-carousel-776

The last angle is a vertical angle (e.g., high angle, eye level, and low angle). (see Figure 1113). This represents power between represented participants and viewers. If the represented participants are depicted from a high angle, viewers seem to have power over the represented participants. In contrast, if the represented participants are seen from a low angle, the represented participants have power over viewers. The eye level angle represents equality between both sides (Unsworth, 2001, p. 98-99).

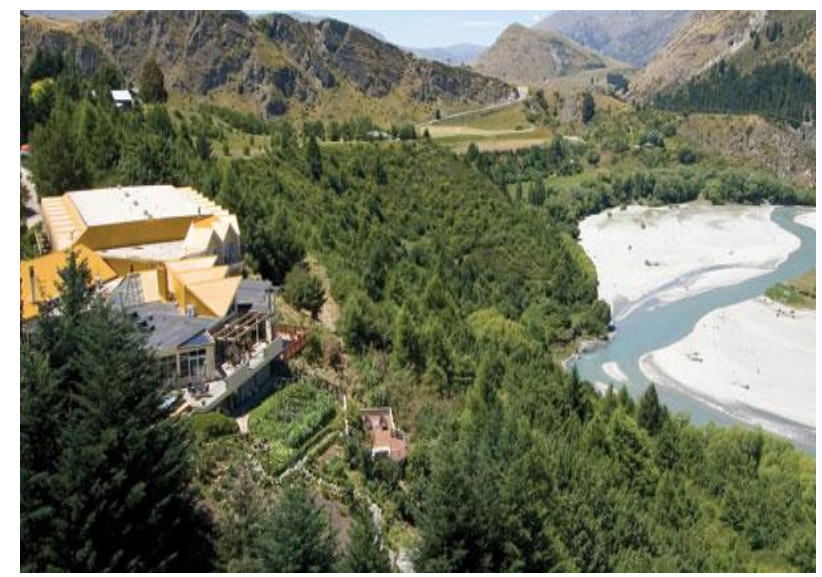

\section{Figure 11: High angle}

Source: http://www.nuggetpoint.co.nz/ 


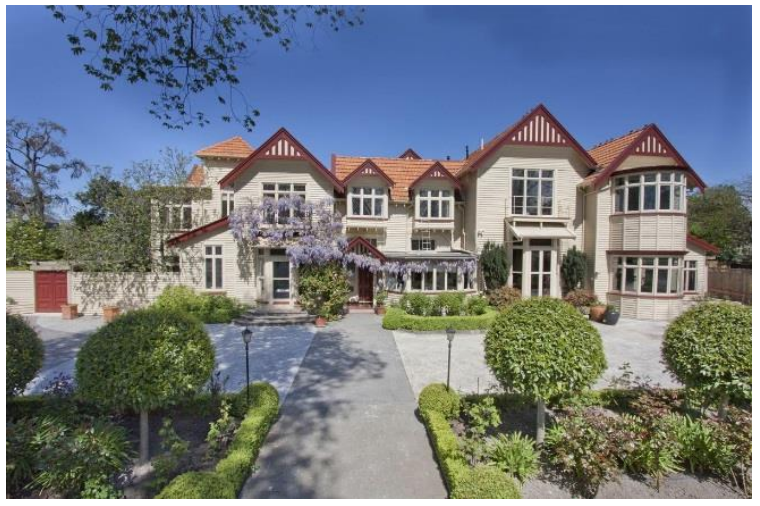

Figure 12: Eye-level angle

Source: http://www.elizas.co.nz/

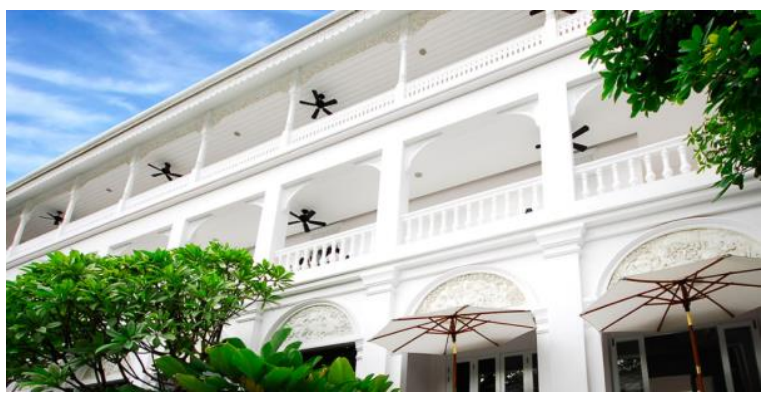

\section{Figure 13: Low angle}

Source: http://www.pingnakara.com/

\section{Compositional meaning}

This meaning is characterised by the integration between three interrelated systems: information value, salience, and framing (Kress and van Leeuwen, 2006). Information value refers to the meaningful placement of visual elements in a composition. Oyama (2001 p. 148) suggests that the left side shows something the viewers already know as familiar content. It is referred to as given. The right side represents the new, something which is not yet known to the viewers; therefore, they should pay attention (see Figure 14). 


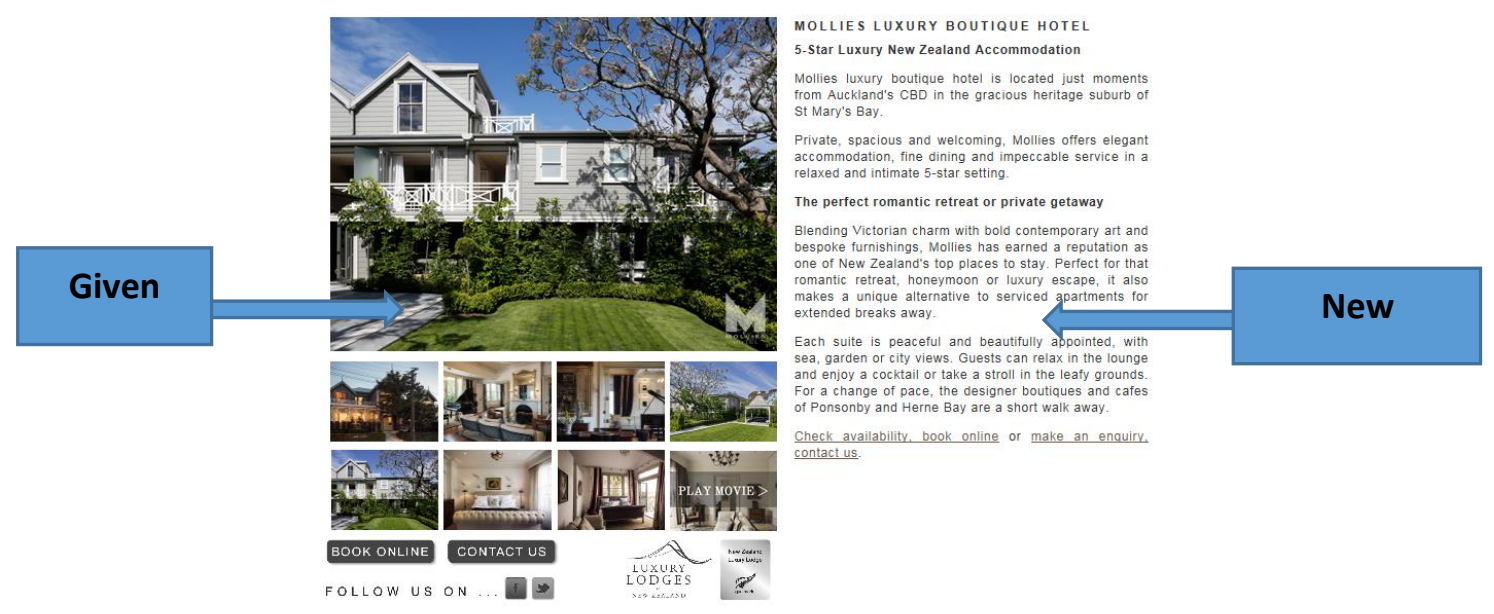

Figure 14: Given and New layout

Source: http://www.mollies.co.nz/about_mollies_luxury_hotel_accommodation.html

The top location implies the most salient or ideal information, and positioning on the bottom refers to real or more specific information (see Figure 15) (Kress and van Leeuwen, 2006). Unsworth (2001, p. 106) and Kress and van Leeuwen (2006, p. 186) suggest that the ideal section in magazines and online advertisements is connected with the promise of a product denoting ideal results. On the other hand, the bottom section typically contains a concrete description of the product, informing the readers about where the product can be purchased and contact details.

The meaning of visual elements can also be identified by the dimensions of centre, the most important part of the image, and margin, those supplementary to the central elements (see Figure 15). In magazines, newspapers, and online advertisements, copywriters frequently combine given and new sections with centre and margin to promote their products and services. This combination of all elements is called a triptych. 


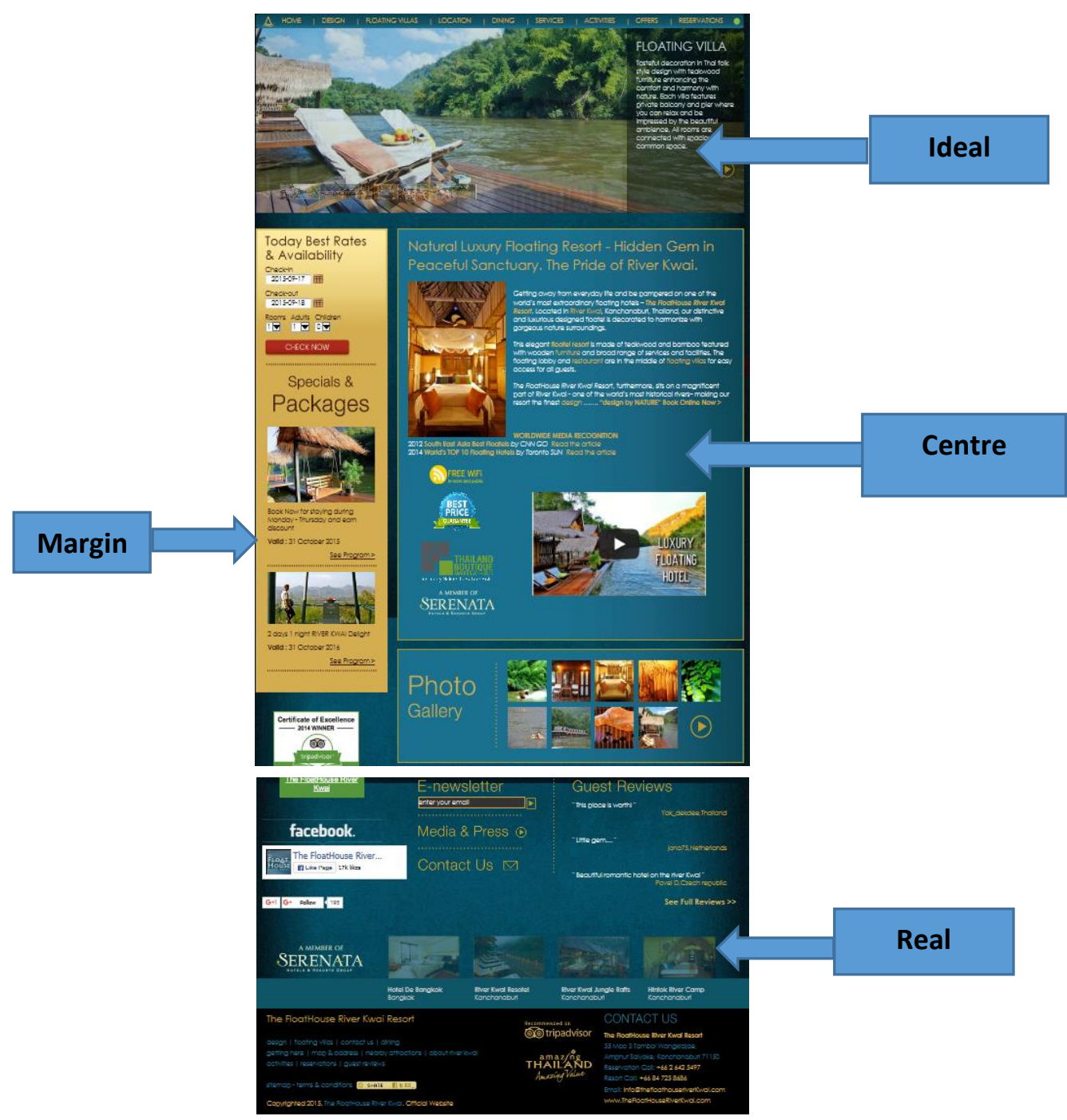

Figure 15: Webpage layout

Source: http://www.mollies.co.nz/about_mollies_luxury_hotel_accommodation.html

This study builds on Kress and van Leeuwen's (2006) work. I applied the aspects discussed above to the analysis of visual images of the twelve boutique hotels in the Thai and New Zealand datasets.

\subsection{Conclusion}

In this chapter, I have outlined the key theoretical concepts that underlie this study. Swales' (1990) and Bhatia's $(1993,2004)$ concept of genre analysis were applied as the principle theoretical framework in this study to explore communicative purposes of hotel webpages and the role of culture in promotional genres. With regard to the representation of visual images on the hotel webpages, I focused on the three metafunctions proposed by Kress and van Leeuwen (2006), which include the visual roles of images (representational meaning), the relationship between represented participants and viewers as well as the persuasive effect conveyed through represented participants 
(interactive meaning), and the layout of the webpages (compositional meaning). Taken together, the strengths of theoretical background and research perspectives from the three main different dimensions (textual elements, visual images, and interviews) are complementary research lenses and approaches that frame my study. Utilising a combination of research perspectives from these three different approaches, I used these research tools to explore the characteristics of hotel webpages as a genre. The next chapter moves to the methodological and practical aspects of this study. 


\section{Chapter 4: METHODOLOGY}

This chapter presents the approach to corpus development and the methodological frameworks employed in this study. This chapter is divided into three main parts, namely the scope of the study, the description of the datasets, and the analytical frameworks.

\subsection{Scope of the study}

The present study explores the genre of boutique hotel webpages which includes the investigation of textual elements, visual images, insiders' perspectives, and web viewers' perceptions. Bhatia's (1993) genre analysis of language in promotional discourse is the main approach employed to examine the genre of boutique hotel webpages. In regard to the textual analysis aspect, I focus on move and register analysis. For the visual images, I adopt Kress and van Leeuwen's (2006) visual analysis model to investigate the visual images represented on the hotel webpages. Regarding the insiders' perspective, interviews with hotel owners, managers, and web designers were conducted to investigate the cross-cultural values influencing the differences and similarities between the way the hotel owners and/or web designers in Thailand and those in New Zealand advertise their websites. Additionally, my study explores how the representation of textual and visual choices on the websites is perceived by the boutique hotel web audience both in Thailand and in New Zealand.

\subsection{Description of the datasets}

This study analyses two datasets of boutique hotel webpages: the Thai and New Zealand datasets. Each dataset comprises 50 hotels, whose three main webpages - Home, Suites, Facilities/ Activities - were selected for the analysis. However, some hotels divide and name their webpages differently according to what services and facilities they aim to promote. Generally, Home and Suites are the main pages that every hotel has, but the organization of facility related pages (e.g., Dining, Spa, Wedding, and Events) varies depending on what each hotel's main focus is. An example can be seen in Figure 16, hotel T8 does not have Facilities/Activities page because their main focus is likely to be on restaurant service and spas. As a result, the hotel separates the Dining and Spa pages to attract the specific group of guests who are interested in those activities. The organisation of T8 hotel webpages, where they have a separate page for Dining and Spa seems to draw attention from guests who would like to spend their holiday in a spa and have a taste of Thai traditional cuisine. In short, my analysis includes three main webpages: Home, Suites, and Facilities/Activities. I also 
incorporate facilities related pages (e.g. Dining, Spa, Wedding, Events) for those hotels with different of webpage organisation.

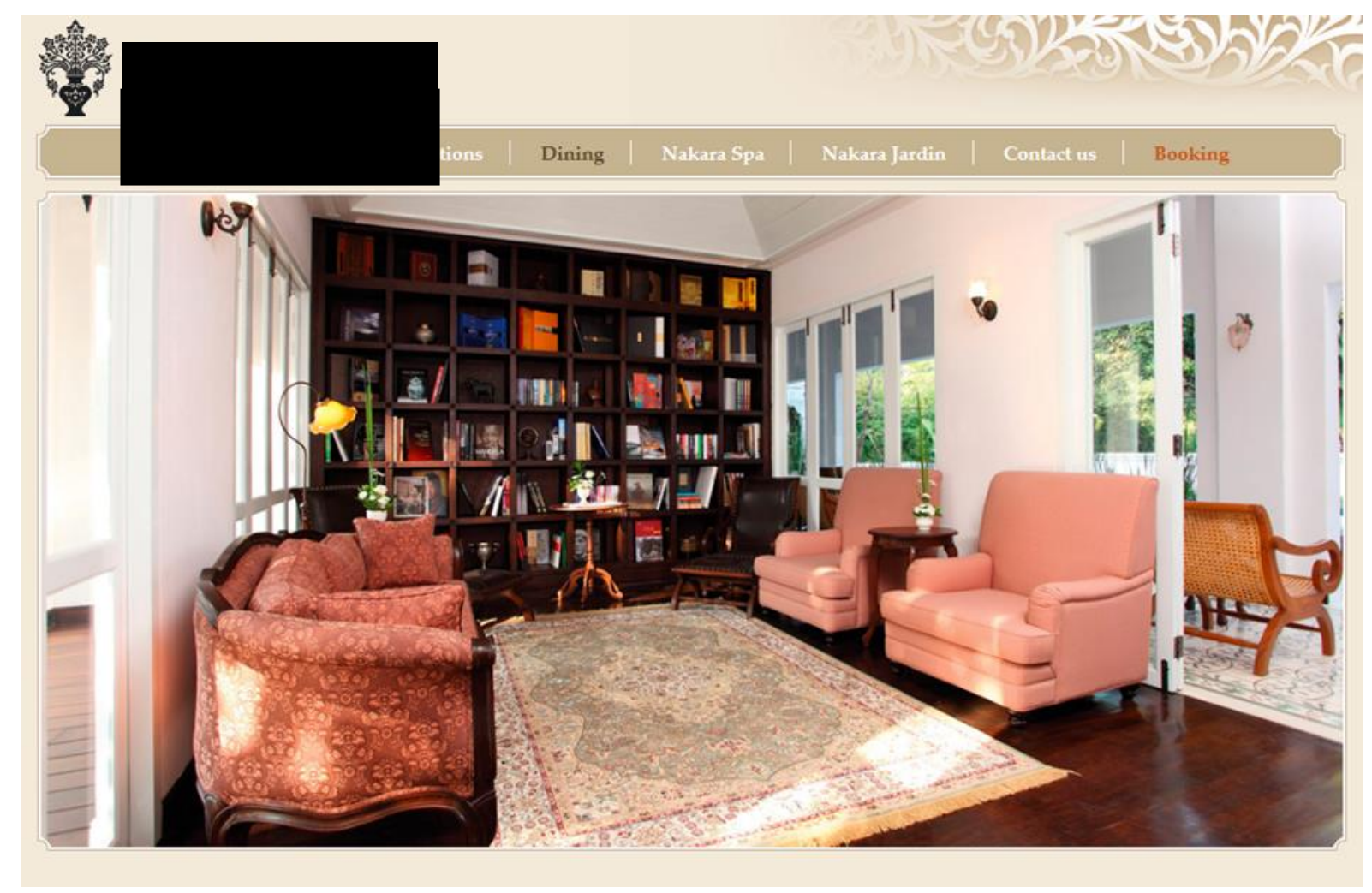

Figure 16: Homepage of T8 hotel

Source: http://www.pingnakara.com/

\subsubsection{Selection of the hotels}

As mentioned above, I selected three main webpages: Home, Suites, Facilities/Activities because they are the most common categories that occur. These three components are likely to describe outstanding characteristics of boutique hotels and contain the majority of words on each hotel website.

The datasets were compiled from March to April 2013. Data were collected from 100 hotel websites in Thailand and New Zealand, which were divided into 50 websites from each country. The websites were selected from the top ten destinations in both countries. The information about the top ten destinations in Thailand and New Zealand was retrieved from the Tourism Authority of Thailand (henceforth TAT) and the Ministry of Business Innovation and Employment (henceforth MBIE) in New Zealand respectively. However, the information about the top ten destinations in 2012 
from TAT was not available during my data collection period. This was because the TAT committee had to sign the document before the release and the staff member could not estimate how long the process would take. As a result, I used the ranking information from 2011, which was the most recent information at that time. In order to ensure that the information I used as a basis for the Thai dataset compilation was not out of date, I then compared the data (2011) that I retrieved from TAT with the ranking categories from the online website Trip advisor and NIDA Poll (see Table 6).

Table 6: Information of the most popular cities in Thailand provided by three different organisations.

\begin{tabular}{|l|l|l|}
\hline TAT (2011) & Trip advisor (2012) & Nida Poll (2012) \\
\hline Bangkok & Bangkok & Chiang Mai \\
\hline Chonburi & Chiang Mai & Phuket \\
\hline Phuket & Krabi & Krabi \\
\hline Ayutthaya & Pattaya-Chonburi & Chiang Rai \\
\hline Kanchanaburi & Patong- Phuket & Chonburi \\
\hline Chiang Mai & Huahin- Prachuap khiri khan & Mae Hongson \\
\hline Rayong & Phuket & Prachuap Khiri Khan \\
\hline Songkhla & Khaolak-Phang Nga & Suratthani \\
\hline Nakon Ratchasima & Karon- Phuket & Rayong \\
\hline Krabi & Bhoput-Suratthani & Loei \\
\hline
\end{tabular}

From Table 6, I selected the cities that occurred at least twice over three polls. These eight cities were Chonburi, Chiang Mai, Phuket, Krabi, Prachuap Khiri Khan, Suratthani, Bangkok, and Rayong. Since I aimed at ten cities I chose two additional cities from the TAT - Kanchanaburi and Nakon Ratchasima - to make up the difference.

For the New Zealand dataset, the ranking information regarding the top ten destinations in 2012 was retrieved from the MBIE. The ten destinations include Auckland, Rotorua, Canterbury, Queenstown, Wellington, West Coast, Taupo, Waikato, Nelson, and Northland.

In this study, the minimum number of boutique hotels required in each region in both countries was five hotels, which made the total of 100 hotels ( 50 hotels in Thailand and 50 in New Zealand). The samples of 100 boutique hotel webpages were selected based on the following 
criteria: the hotels must have their own official websites, which consist of at least three main pages: Home, Suites, Facilities/ Activities or equivalent (discussed in section 4.2: Description of the datasets). In order to retrieve the names of boutique hotels, I used the search engine Google to find the information on the World Wide Web. I searched the boutique hotel websites by using the keywords boutique hotel + the name of each city (boutique hotel in Bangkok). However, in some cities, such as Nakhon Ratchasrima and Rayong, it was difficult to find five official hotel websites. Mostly the search results on search engine Google were web directories (e.g., trip advisor.com, agoda.com). In order to obtain a larger number of hotel names, I used the search results from the web directories. After that, I used hotel names from the directories to find their official websites. Even though some of the hotels would not like to reveal their name and identity, they allowed me to use their webpages for my analysis. In order to protect their identity, I used alphabetised and numerical labels to refer to those hotels (e.g., T1 and NZ1).

Nvivo 10 was used to save the hotel websites. Nvivo10 is software that can download webpages, including visual images, sounds and other media files. This step enabled me to browse the site offline while making sure no content or structural changes would occur during the data collection. The data of the hotel webpages used for this study consists of texts and images.

\subsection{Analytical frameworks}

A key element of the study's approach was to integrate textual and visual elements, as well as interviews to gain a rich understanding of how and why boutique hotels construct their websites the way they do. The comparison of textual and visual elements of the Thai and New Zealand datasets was then used to identify dissimilarities between the content of the webpages and their writing style. The data analysis of this study was conducted by using the following procedures: move/ step analysis, register analysis, content analysis, visual image analysis, and interviews.

\subsubsection{Move/step analysis}

Bhatia's (1993) genre analysis was adopted to identify move structures of three main webpages Home, Suites, Facilities/Activities of 30 selected hotels in the Thai dataset and 30 in the New Zealand dataset. A move in this study refers to "rhetorical instruments that realise a sub-set of specific communicative purposes associated with a genre" (Bhatia, 2001, p. 84). A step is defined as a strategy (Bhatia, 1993; Henry \& Roseberry, 2001) or rhetorical options, which discourse members can select to realise moves. Even though move analysis does not have explicit rules, move identification in this study was based on the meaning of the texts in the discourse community where 
the genre is situated, the communicative purposes (or the web designers' intent), and linguistic evidence. The details of how I identified moves and steps are discussed in Chapter 5 section 5.1.

After the identification of moves, the frequency of occurrence was explored in order to determine whether the moves were obligatory or optional on each webpage. I selected the cut-off frequency at $75 \%$ based on Mulken and van der Meer's (2005, p. 100) proposition to identify whether the move structures in my study are obligatory or optional. Moves that occurred in $75 \%$ or more were defined as obligatory moves, but those that occurred in less than $75 \%$ of texts were defined as optional or subsidiary (Mulken \& van der Meer, 2005 p. 100). I was aware that the cut-off frequency of move structures varies according to study (the detailed discussion is in section 3.1 Chapter 3: Theory). I selected the cut-off frequency at $75 \%$ because I did not want to limit the scope of the frequency of occurrence of moves in my analysis. To test whether the $75 \%$ cover the main communicative purposes of my dataset, I did a preliminarily analysis and discussed the findings with my supervisors. To identify moves, I reflected on the overall intention that hotels try to communicate to the readers and examined the recurrence of distinct lexico-grammatical features that the hotels use to serve each intention.

\subsubsection{Register analysis}

The investigation of the distinctive characteristics of the lexico- grammatical features of each move used corpus linguistics methodologies, namely, word frequency counts and concordancing. The analysis was conducted by using a computer program called Antconc 3.2.4. This program comprises three main analytical tools: Wordlist, Keywords, and Concord. Wordlist presents the frequency of words. Concord shows the collocation of words. Only Wordlist and Concord were adopted to investigate the prevailing linguistic features in this study. Specifically, at a register level, I examined imperatives, personal pronouns, modal verbs, and adjectives. I selected these five features for my analysis because they are common features that have been previously studied in tourism materials. In addition, these features commonly occurred in my dataset. Since the main purpose of tourism discourse is to persuade target readers to buy and use products and services, the language used in this discourse reflects persuasive functions and shares certain similar features. For example, the frequent use of adjectives to describe products and services, and personal pronouns (we and our) to make the target readers feel that the hotels share a similar idea or goal with the readers (Maci, 2007; Pierini, 2009).

To provide data for analysis, I collected data in electronic format taking into account its representativeness of boutique hotel webpages in Thailand and in New Zealand. This dataset represents 61,457 words. The frequency of wordlist and the collocations of the distinctive 
features were generated by AntConc 3.2.4. The selected frequent linguistic features (imperatives, passive voice, and pronouns) in this study are based on typical linguistic features that are found in the previous studies concerning promotional genre (Maci, 2007; Pierini, 2009). In addition, to confirm that these features are also distinctive in my study, I observed their frequency of occurrence and made sure that they were a common feature that occurred in the frequency wordlist.

\subsubsection{Visual design analysis}

In this study, I adopted two stages of visual images analysis: 4.3.3.1 content analysis and 4.3.3.2 grammar of visual design (Kress and van Leeuwen, 2006).

\subsubsection{Content analysis}

I adopted content analysis model to examine the characteristics of visual images and categorise these depicted on the boutique hotel webpages. The purpose of using this approach was to minimise the dataset size that I used for move analysis and visual image analysis. This approach involved counting and categorising the number of facility images depicted on the suite/accommodation pages of boutique hotel websites to retrieve representative websites from the whole population. Based on the categorisation showing three different aspects - the highest number, the average number, and the smallest number of facility images - I selected ten hotels from each category, resulting in 30 hotels in total in each dataset (the Thai and New Zealand datasets). That is to say, based on the content analysis, I obtained 30 hotels, which were representative of each country. These hotels were selected to analyse move structures and lexicogrammatical features. The reason that I had to minimise my dataset was that move analysis and register analysis are time consuming and it is more feasible to focus on 30 websites. In regard to visual image analysis, since each website contains a large number of images, I could not include all 60 websites. I chose six hotels from each dataset (12 in total) based on the geographical proximity between the hotels for ease of transportation. That was because I aimed to interview the owners and/or staff members of these hotels after the textual and visual analysis.

\subsubsection{Visual image analysis}

In order to investigate visuals in a systematic way, I applied Kress and van Leeuwen's (2006) framework, which they call "Visual grammar", to examine how the images were presented on the hotel webpages and how they interact with the viewers. Since Kress and Van Leeuwen's (2006) framework is comprehensive and culturally specific, I adopted three main metafunctions Representational meaning, Interactive meaning, and Compositional meaning - of the framework but 
adapted them to suit my study context (see Figure 17). This framework was used to answer my research questions: 1) What are the features of visual images in English boutique hotel websites in Thailand and in New Zealand? 2) What are the similarities and differences between boutique hotel websites in Thailand and in New Zealand? In this study, I applied the aspects discussed above to the analysis of visual images of the twelve boutique hotels in the Thai and New Zealand datasets. As I have observed, hotel and tourism promotional materials usually contain images of scenery, buildings, and human. In this sense, the images can be interpreted as inviting the viewers to enter the scenes depicted. If the images depict human beings, they enhance the relationship between the hosts and visitors (Franscesconi, 2014). 


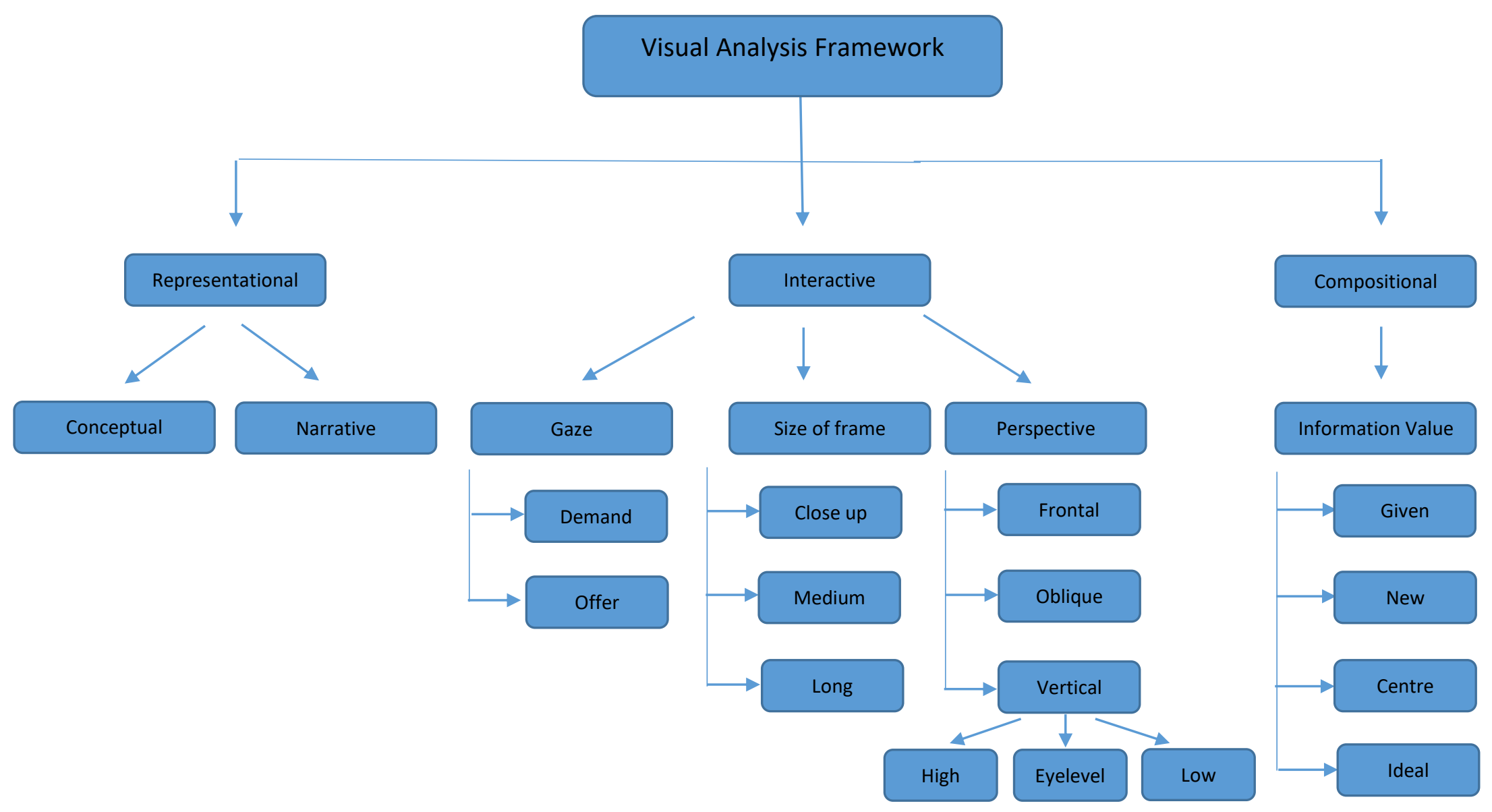

Figure 17: The visual analytical tools adapted from the grammar of visual design (Kress and van Leeuwen, 2006) 


\subsubsection{Interviews}

This section discusses the interview procedures that I used for an investigation of the insiders' perspectives of hotel websites design. This study draws on interview data and the previous literature to understand the characteristics of boutique hotel webpages and their context. During the textual and visual examination of my data, a number of questions arose that could not be answered by the analysis alone. I adopted a semi-structured interview approach to gain insight into the questions. My questions focused on the characteristics of boutique hotel webpages and contextual factors influencing the decision on how and why the hotels used the language and visual images they did.

However, there were two major drawbacks that arose during the interviews. First, the information from the participants was influenced by the contexts in which the interviews took place. As a result, I became aware that the information that I obtained from my participants may not have truly reflected what the participants were thinking in the interviews. For example, when I asked the hotel owners how they developed their promotional texts, some of them told me that they had consulted other hotel websites to get ideas for the production of their texts. The consultation of other hotel websites may influence the similarities in hotel websites in terms of rhetorical structures and distinctive lexico-grammatical features across my dataset. Secondly, there were certain questions which in hindsight I would have liked to ask. However, since they were one-off interviews, I did not have a chance to ask those questions. I emailed my participants but some of them did not reply. However, interviews were the most feasible approach to getting information from insiders since the participant observation approach was not practical in the context of my study.

All the interviews were tape-recorded. Voice-recordings allowed me to interact freely with the participants without worrying about missing out important information. I did not make complete transcriptions of all interviews. I listened to the recordings and made detailed summaries of the conversation after each interview in order to highlight the information most relevant to my research purposes. I only transcribed the sections that were directly related to my questions. After that, I analysed and gained understanding of the distinctive features of the hotels, the contexts, and the practices of the hotel operators by reading and re-reading the notes again. I looked for the themes, key words, repetitive patterns, and different responses across the notes.

There is no separate chapter of the analysis for the interview data. The responses, comments, and opinions of the participants are described and are integrated in the analysis of move structures (chapter 5 move analysis), distinctive lexico-grammatical features (chapter 6 register 
analysis), and visual images (chapter 7 visual design). I drew on the interviews in order to clarify the analysis and support my findings.

\subsubsection{Interviews with hotel owners/web designers}

A regional selection of the hotels offered the most appropriate sampling approach for this study. I aimed to interview the twelve hotel owners/staff members that I selected following the content analysis (see section 4.3.3.1 Content analysis) for my visual analysis because I could ask my respondents specific questions that were related to the content and the design of the webpages that I had explored. I emailed these twelve hotels to request an interview, attaching a letter of consent and an information sheet in the email. I received a response from only five hotels out of six on the list from the New Zealand dataset and none from the Thai dataset. However, in the New Zealand dataset, only three hotels out of the five that had responded stated that they could take part in my study.

One of the two that were not willing to participate in my research was a new management team that had just taken over the hotel. They noted that they would not be able to help me with the information that I needed regarding the hotel website. The owner of the other hotel, which did not take part in my study explained the reason that he could not participate in my research because he was going to be travelling aboard during my data collection period. I speculated that the low response rate from the hotel business sector may have been because it was approaching the peak tourist season in both countries at the requested interview time. The difficulties in recruiting target participants and encouraging them to participate in research seem to be the most common drawback for ethnographic approaches as suggested by Cook, Reed and Twiner (2009) and Mcintosh and Siggs (2005).

In order to gain more responses from the hotels in both countries, I emailed every hotel from the top ten destinations list (see section 3.2.1 Selection of the hotels). However, in order to manage my interview trip cost-efficiently, I limited my selection to the regions that were geographically proximal for ease of transportation. These regions from the Thai dataset were Bangkok, Chonburi, Prachuap Khirikhan, Rayong, Kanchanaburi, and Nakon Ratchasima. The New Zealand regions were Wellington, Auckland, Northland, Rotorua, Taupo, Canterbury, and Queenstown. As a result of this email, I obtained two more responses from hotel owners in New Zealand agreeing to participate in my study. The final samples in the New Zealand dataset included five out of six hotels from the list of the hotels that I used for my visual analysis: two hotels in Auckland, one in Waiheke Island, one in Rotorua, and another one in Canterbury. For these five hotels, my respondents were couples who were owner-operators. For the first three hotels, I 
interviewed only the wives. In the fourth hotel, at first, I interviewed the husband and then the wife participated later because she was checking a guest in when I arrived at the hotel.

There was one participant who had designed his website by himself in terms of the selection of visual images and the use of languages. The other four participants had had web designers produce the website, although they maintained and updated the website themselves. The interviews with the hotel owners were conducted in their hotels between September and October 2014. The duration of each interview and the associated accommodation tour was approximately 30-35 minutes each.

In regard to the data collection for the Thai hotels, I did not receive any responses from the hotels in the Thai dataset from my first or follow up emails. However, when I was in Thailand for participant recruitment, I saw an advertisement for the Thailand boutique hotels award, where my friend was the master of ceremonies. This event was organised by a well-established bank in Thailand and its alliances. After that, I contacted my friend, and she introduced me to the organising committee of the Thailand boutique hotels award. Through this introduction, twelve hotels agreed to participate in my study. These hotels were in Bangkok (two hotels), Chiang Mai (five hotels), Kanchanaburi (three hotels), and Rayong (two hotels). Even though only six hotels were the hotels that I had chosen for my visual analysis, the data from the boutique hotels whose websites I did not analyse was still helpful in terms of the information of how they position themselves as a boutique hotel. Out of twelve hotels, I conducted face-to face interviews with five hotel managers and three hotel owners. I interviewed the rest of them over the phone. Of the four participants interviewed over the phone, two were hotel owners and two were hotel managers. The reason that I had to interview these participants on the phone was because my data collection period coincided with festive period in Thailand and my participants were not available for face to face interviews.

There were only three hotels (two hotels in Thailand and one hotel in New Zealand) that had taken part in designing their hotel websites. The other hotels had had web designers and copywriters make their websites. Those who had not taken part in designing their own website, sometimes could not comment or answer my questions related to the representation of the webpages. The face -to-face interview duration and the hotel tour took about 30-40 minutes and were tape-recorded. The interviews on the phone took about 25 minutes. The interviews were conducted in Thai except for one hotel, where the hotel manager was a German; that interview was in English. 
The representative attributes of the hotels were identified from the interviews. These features were informed by the notes I made after interviews. All five hotels in New Zealand were owner-operated, and three of them were also the host's private property. The number of guestrooms ranged from 4 to 10 rooms. Two hotels had been converted from historic buildings. On the other hand, of the boutique hotels in the Thai dataset, ten hotels were operated in the form of a company, and two were owner-operated by companies. Of the twelve hotels, five owners had more than one hotel in their company; some were boutique hotels and some were other types of hotels. Only two of them were converted from old historic houses. The number of suites in each hotel was seven to 45 rooms.

The questions began with the interviewees' scope of work/main responsibilities; their experience of hosting, how they define boutique hotels; and what key characteristics that they used to position their hotels as boutique hotels. They were asked who designed the websites; who was involved in making decisions; what were the functions and who were the audience of the websites; and what were their marketing strategies. I had a pre-formulated question list (see Appendix 5) but the questions and their sequence varied based on the responses that I received from each interviewee. The questions related to language and visual images of hotel webpages were about the design process, the purpose, the conventions and practices, and specific questions based on the representation of the websites such as what does the hotel logo represent? and Why does the hotel use the colour brown for the background of the webpages?

In regard to the interviews with the web designers, I had three web designers participating in my study, all of whom were introduced by my friend, who was also a professional web designer. Each of them has more than ten years' experience in designing hotel websites. Additionally, one of them had designed boutique hotel websites for more than a hundred hotels. Apart from these three web designers, one of the hotel owners also designed his hotel websites. In addition to the perspectives toward his boutique hotel in general, he could also clarify the questions that were related to the website design. In my analysis, in order to protect their identity, I used a code to refer to them e.g., web designer 1, web designer 2. Each interview lasted for about 40 to 60 minutes and was tape-recorded. The interviews began with the web designers' scope of work, the procedures of designing hotel websites, and the guidelines/principles for designing websites. After that, the web designers showed websites that they had designed, and based on the websites I asked them specific questions related to the language used and visual images depicted on their websites. The questions varied in each interview, depending on the websites that they showed to me and their experiences in designing websites. 


\subsubsection{Interviews with hotel website audience}

The main purpose of these interviews was to understand the web readers' perceptions of boutique hotels in general, and, more specifically, the language and design of the webpages that I used for my data analysis. Additionally, I aimed to explore if there was a mismatch between the communicative purposes, including persuasive effects, that the boutique hotels intend to convey and the perceptions that the audience has when reading their websites.

The information from interviews with the three web designers revealed that the decision on the design of websites is primarily based on the users' preferences. As a result, ten web readers in Thailand and eight in New Zealand were recruited by the snowball sampling strategy (Sadler, Lee, Lim, \& Fullerton, 2010) in order to fulfil this research purpose. The sampling technique enabled me to overcome the challenge of recruiting the specific desired participants that I set for my study. The information of the hotel guests, who are ideally my participants cannot be revealed by the hotels because of policy protecting guests' privacy. As a result, I used snowball sampling. This approach obtains participants who are otherwise difficult to reach by using people's social networks to recruit similar participant to the specific target group (Sadler, Lee, Lim \& Fullerton, 2010). The snowball strategy enabled me to recruit my participants through my friends' networks. I started by interviewing my friend who had had a lot of experience staying in boutique hotels. After that she introduced me to her friends who had also stayed in boutique hotels.

My target participants were people who had had experience of staying in a boutique hotel or at least understood the concept of boutique hotels. The participants were predominantly females aged 30-40. In this way the sample reflected an underrepresentation of male respondents. The web audiences were diverse such as housewife, employees, business owners, and students. The interviews began with a question about the web audience's previous experience in boutique hotels and moved on to what attributes they expect from staying at boutique hotels; how they made a decision when booking a boutique hotel, and the purpose of stay (see appendix 6). After that, each participant was shown two webpages out of twelve from both datasets that I had analysed, and was asked to evaluate the contents (language and images) of the webpages. The scope of questions depends on how comfortable the Thai participants were in answering given that the websites were written in English. During my web audience member recruiting process, I had informed the participants that they might be asked questions related to the language used on the webpages which were written in English. However, during the interview, several of the Thai participants did not seem to be comfortable commenting on the language. At that point, I stopped asking them questions. In regard to the web audience in New Zealand, only four of them spoke English as their 
first language. With the other four, English was their second language. Even though all of my participants in the New Zealand dataset spoke English fluently, they were reluctant to evaluate or comment on the use of language that was presented on the websites.

The web audience interviews had a number of limitations. One drawback, referred to above, was no insightful opinions towards the use of language was obtained. Secondly, I had no access of actual guests due to the protection of confidential information of the guests. Another disadvantage was the environment and the procedures of the interviews were artificial. The participants were not real guests and they did not have an intention to book a hotel. As a result, the social purpose of visiting hotel webpages in order to make a reservation was absent. Furthermore, some interviewees in New Zealand had not had experience staying in a boutique hotel, so the concept of boutique hotels was not clear to them. The definition of boutique hotel is not clear-cut. The interviewees may have confused boutique hotels with bed and breakfasts.

\subsection{Conclusion}

This chapter has outlined the research scope, methodological framework, the description of the dataset, and the method of data analysis that I used to answer my research questions. The similarities and differences between Thai and New Zealand datasets in terms of textual and visual elements were applied to identify possible factors that influence the webpage variation between the Thai and New Zealand dataset. The combination of genre analysis and interviews aimed to build rich descriptions and understandings of boutique hotel web advertising as a promotional genre and the context around it. 


\section{Chapter 5: MOVE ANALYSIS}

This chapter presents the move structure of the three main webpages - Home, Suite, and Facility/Activities - of the 30 boutique hotel websites in Thailand and the 30 in New Zealand. It describes the identification of moves and steps in the boutique hotel websites of the Thai and New Zealand datasets by using the genre analysis frameworks of Swales (1990) and Bhatia (1993).

Swales (1990) views a genre as a group of communicative events in which similar forms of language are used as a representation of shared communicative purposes realised by members of the same discourse community (p. 47). Drawing on the concept of shared communicative purposes, he proposes move analysis as a key for identifying a genre. Following Swales (1990) and Bhatia (1993)'s framework, the identification of moves and steps in my dataset is based on the analysis of communicative purposes, linguistic evidence, and comprehension of the hotel advertising discourse. According to Swales (1990), a move is a segment of a text that performs a particular communicative purpose. Moves that occur in $75 \%$ or more of texts in a dataset are obligatory moves, but they are optional if they occur less than $75 \%$ in frequency (Mulken \& van der Meer, 2005). Bhatia (1993) pays attention to the significance of socio-cultural aspects of a genre in order to answer the question of why the texts are written the way they are. While moves are discourse units that realise communicative purposes, the term steps refers to strategies (Bhatia, 1993; Henry \& Roseberry, 2001), or rhetorical options which discourse members can select to realise moves. Henry and Roseberry (2001) suggest that there are certain textual patterns which are frequently associated with specific moves and steps. However, Bonyadi (2012) claimed some moves may be realised through a variety of steps or strategies. In her study of newspaper editorials, the move orienting the reader exhibits two different linguistic realisations: a declarative sentence and a rhetorical question.

As I mentioned earlier in Chapter 2, section 3.1, Swales (1990) and Bhatia (1993) argue that writers use a step/steps to realise moves but both of these scholars fail to explain the difference between a move and a step. I found no study suggesting principles to distinguish between moves and steps. In my move analysis, I read through each page (Home, Suite, and Facilities/Activities page) to understand and get the overall idea of each page and I looked for linguistic signals such as paragraphing. Moves are identified based on the communicative purposes that hotels aim to convey to the readers. Steps are considered as options writers select from to achieve the moves. What distinguishes a move from a step is that a move has a higher structural status than a step. It reflects 
a hierarchical difference in communicative purposes between moves and steps. For example, for the move Establishing credentials, there are eight rhetorical steps that the writers can choose to achieve the function of this move. These steps include Describing location, Claiming superiority, Claiming luxury, and Introducing the external awards received. These steps occur under the umbrella of the move. The steps reflect the hierachical structure of the intentions between the steps and the moves, and the steps support to the realisation of the move Establishing credentials. In short, discourse units that convey the main communicative purpose are moves and those that express subordinate functions are considered as steps.

\subsection{Identification of Moves and Steps in Thai and New Zealand boutique hotel webpages}

In this section, the move structures of these hotel webpages are first outlined (Table 7) in terms of the types of moves, whether the moves are obligatory or optional by focusing on the frequencies of occurrence of each move. After characterising each move, I described and exemplified each step. Moves are identified based upon the communicative purposes that they perform. The names of the moves indicate their functions on the hotel webpages. The communicative function of each move is described in the following section. After identifying moves, I identified the steps that the hotels use to achieve a particular move or function inthe texts. The move and step identification process is difficult since there are no explicit guidelines to follow. I repeatedly reviewed and revised the move/step patterns based on my comprehension of the texts and lexico-grammatical evidence. In addition, I drew on the information from the interviews with the hotel owners, web designers, and web readers to guide and validate my analysis.

Ten moves were identified in the Thai dataset and eleven in the New Zealand dataset (Table 7) after analysing to get an overall idea of the three main webpages. Moves which occur more than 23 times (75\%) are identified as obligatory moves (shown in bold in Table 7), and lower than 23 times are optional. Even though Thai and New Zealand datasets share the same total number of obligatory moves (4 moves), both countries adopt only three similar moves to advertise their hotels (Move I: Introducing the hotel/facilities/services, Move DR: Describing the rooms, and Move E: Establishing credentials). Six moves out of ten are optional moves in the Thai hotel webpages, while seven out of eleven are optional moves in the New Zealand dataset. The discussion of obligatory moves, their steps, and examples follows. 
Table 7: Identification of Moves in Thai and New Zealand boutique hotel webpages

\begin{tabular}{|c|c|c|c|c|}
\hline Moves & $\begin{array}{c}\text { Thailand } \\
\text { hotels } \\
\text { (Total 30) }\end{array}$ & $\begin{array}{l}\text { Obligatory/ } \\
\text { Optional }\end{array}$ & $\begin{array}{c}\text { New } \\
\text { Zealand } \\
\text { hotels } \\
\text { (Total 30) }\end{array}$ & $\begin{array}{l}\text { Obligatory/ } \\
\text { Optional }\end{array}$ \\
\hline $\begin{array}{l}\text { Move I: Introducing the } \\
\text { hotel/facilities/services }\end{array}$ & 30 & Obligatory & 27 & Obligatory \\
\hline Move DR: Describing the rooms & 30 & Obligatory & 30 & Obligatory \\
\hline Move E: Establishing credentials & 26 & Obligatory & 25 & Obligatory \\
\hline Move DO: Describing the offers & 28 & Obligatory & 22 & Optional \\
\hline Move C: Calling for action & 20 & Optional & 24 & Obligatory \\
\hline $\begin{array}{l}\text { Move R: Introducing restaurants/bars, and } \\
\text { lounges }\end{array}$ & 22 & Optional & 15 & Optional \\
\hline Move V: Introducing function venues & 8 & Optional & 12 & Optional \\
\hline $\begin{array}{l}\text { Move S: Showing environmental and local } \\
\text { community support }\end{array}$ & 1 & Optional & 3 & Optional \\
\hline Move T: Indicating Terms and Conditions & 7 & Optional & 6 & Optional \\
\hline Move F: Friendly ending & 2 & Optional & 5 & Optional \\
\hline $\begin{array}{l}\text { Move Q: Introducing Frequently Asked } \\
\text { Questions }\end{array}$ & 0 & Optional & 2 & Optional \\
\hline
\end{tabular}

\section{1) Move DR: Describing the rooms}

This move is an obligatory move which occurs in every text in my dataset. This may be because the primary purpose of hotel business is to sell their accommodation. The information related to accommodation is vital for the readers to make a decision to book the rooms. The main function of this move is to introduce the readers to the types of hotel rooms offered, the facilities, the services as well as complementary gifts provided, the atmosphere, the design and decoration (theme), including the outdoor area and the view of the rooms. Also the purpose is to persuade them to stay in this accommodation. This move can be realised by the four steps as shown below which are followed with explanations and detailed examples.

Step df: Describing the rooms facilities/ the outdoor area and the view of the rooms

Step ar: Describing the atmosphere and the design of the rooms (theme of the hotel suites)

Step pr: Stating the price of the rooms for individuals and groups 
Step gc: Identifying target guests/capacity of the accommodation

In my dataset, some sentences integrate instances of both step df (Describing the rooms facilities/ the outdoor area and the view of the rooms) and step ar (Describing the atmosphere and the design of the rooms). An example of this integration will be shown after the examples of "step ar."

A) Step df: Describing the rooms facilities/the outdoor area and the view of the rooms

Every hotel in my dataset uses this step in Move: (DR) to fulfil their intention to promote their rooms. The function of this step is to give the readers information regarding the types of accommodation, facilities/amenities, and complementary gifts that are provided in the rooms. In addition, it highlights the dominant features and benefits offered in the room. It also describes the location and the exterior views that the guests can enjoy while staying in the room.

Examples:

1) Club Bamboo offers 46 comfortable accommodations, with eight different types of rooms. This accommodation in Patong is situated in and around the garden, linked by steps and side paths; providing intimacy, sophistication and a warm stay in the resort with individual attention, typical to small resorts (T10).

2) All hotel's guest rooms and villas are exceptionally large and offer splendid sea view. The hotel's serene ambience, together with spectacular sea view and pristine landscape, ensures an ideal romantic getaway and your memorable seaside vacation (T1).

3) All our rooms offer individual fireplaces, complimentary WiFi, air conditioning, in-room safes, mini bars, high definition flat screen TVs, iPod dock stations, DVD players, espresso machines, high quality linen, feather comforters \& pillows, Citta Design bathrobes, Aromatherapy bath products, monsoon rain showers, solid stone soaker baths, heated mirrors and fully tiled bathrooms with under-floor heating (NZ5).

4) Each one of the four ensuite rooms offers a feeling of style and is complemented with a luxury bathroom. After a deep, relaxing sleep in pillow-top beds you can enjoy a superb a la carte breakfast prepared to your taste (NZ19). 
B) Step ar: Describing the atmosphere and the design of the rooms (theme of the hotel suites)

The purpose of this step is to create a desire in the viewers to stay in the hotel through an illustration of the hotel atmosphere and its design.

Example:

1) Room interiors, although enjoying the entire contemporary style that you would expect. We cleverly retain the local culture heritage with traditional interpretations of Thai Local style, featuring quality natural materials and exotic carved hardwood skillfully incorporate into each design (T3).

2) Rich in decor and lined with quality hardwood floors, the 40 sqm. room projects quality for value (T7).

3) This room is in the colour of the sea and sky. There is a series of photographs on the walls depicting TE MOANA taken on the Coromandel Coast by Jonathan (NZ8).

4) Our Cottage is newly-built (completed in 2009), custom-designed, and made of eco-friendly solid timber (sourced from sustainable pine forests on the North Island of New Zealand) (NZ3).

The following is the examples showing the integration between step " $\mathrm{df}$ " and "ar" when the hotels presenting the description of the rooms.

Examples:

1) Accommodation: Le Vimarn has been created in the style of a tropical hillside village (step: ar) with $\mathbf{3 1}$ units comprised of Deluxe Cottages, and Villas with a private spa (step: $\mathrm{df}$ ). Each unit is handsomely decorated in a distinctive island style (step: ar), convenient and supremely comfortable. Teak rattan, bamboo and Thai woven fabrics create a charming ambience (step: ar) which you will appreciate (T17).

2) There is a king/twin bed (step: df), the Victorian furnishings complement the suite as does the three limited editions by Cleavin, renowned New Zealand artist (step: ar) (NZ7). 
C) Step pr: Stating the price of the rooms for individuals and groups

This step provides guests with information about the price of accommodation offered which is usually presented in a noun phrase.

Examples:

1) Thirty eight (38) rooms and four(4) room types starting as low as $\mathbf{5 5 0 0}$

Thai Baht per night are available to our guests. Rates are seasonally adjusted and several promotions are available during the year (T7).

2) deluxe balcony room from 7260 BAHT (T18)

3) Tariff: From $\$ \mathbf{1 6 0 . 0 0}$ inc GST per night (NZ22)

4) River Room Rate: \$170 - \$230 (NZ23)

D) Step tg: Identifying target guests/capacity of the accommodation

The main purpose of this step is to inform the readers about the target guests that the hotels aim to market. In addition, the hotels provide the information about their capacity for different kinds of accommodation. This step is realised by the use of verb phrase: is ideal for, is perfect for, suitable for, and is good for.

Examples:

1) It is perfect for honeymooners, as well as leisure travelers who have discerning taste for an exotic blend of neo-eastern ambience (T7).

2) Suitable for a family of 4 (T10)

3) This suite is ideal for family holidays, groups or business people traveling together (NZ22).

4) Our luxury Queenstown accommodation's Two-Bedroom Suite is ideal for families with up to two children (NZ15). 


\section{Move: I Introducing the hotel/facilities/services}

This move is predominant in hotel web advertising as it occurs in all 30 texts in the Thai datasetand 27 texts in the New Zealand dataset. The function of this move is to introduce the hotel/facilities/services to the readers. This move can be realised by three steps:

Step ns: Stating the name/ or slogan of the hotels

Step gr: Greeting/welcoming the readers

Step ph: Providing a heading for different aspects of the hotels

\section{A) Step ns: Stating the name/or slogan of the hotels}

This step informs the readers of the name and slogan of the hotels which establish the identity of the hotel. The hotels use a slogan to communicate their characteristics and major selling points to the readers.

Examples:

1) Beyond the sense (slogan)

Devasom (name) Hua Hin Resort

Hua Hin Luxury Boutique hotel (T1)

2) Natural Luxury Floating Resort - Hidden Gem in Peaceful Sanctuary. The Pride of River Kwai (slogan) (T2)

3) Rive Gauche Boutique B\&B and self-contained accommodation in Taupo New Zealand (name) (NZ1)

4) Luxury Boutique Accommodation Auckland - Seafields

Bed \& Breakfast (name) (NZ2)

\section{B) Step gr: Greeting /welcoming the readers}

This step identifies a welcoming statement to the readers. It is usually realised by the word welcome and invite followed by the name of the hotel or facilities and services that the hosts aim to encourage the readers. Welcome (69\%) occurs more frequently than invite 
(31\%) in both Thai and New Zealand datasets. This step also highlights the main marketing messages and creates the characteristics of the hotels to the readers.

Examples:

1) Welcome to Devasom Hua Hin Resort, a luxury boutique beach hotel where romantic Hua Hin charms and seaside serenity unite (T1)

2) We cordially invite you to our unique Hua Hin hotel on a private beach with 24 luxury guest rooms and villas in charmingly refined style, inspired by Hua Hin beach houses of Siamese era (T1)

3) However you say Haere Mai!

Welcome to one of the prettiest views in New Zealand - overlooking the beautiful Motueka River and out across Tasman Bay (NZ3)

4) Your hosts Nick and Jenny invite you to stay and share with them the intriguing history, impressive architectural features and the graceful ambiance of times past in one of Nelson's most fascinating buildings. (NZ4)

\section{C) Step ph: Providing a heading for different aspects of the hotels}

This step is a structural step which is realised by a noun phrase introducing the information that follows. It performs its function as a title/heading of a section. It is usually located at the beginning of a new paragraph or a new section. It is used consistently throughout the three pages: Home, Suite, and Facilities and Services.

Examples:

1) Dhivarin Spa (T17)

2) ROOM FACILITIES (T3)

3) Restaurant \& Spa (NZ4)

4) Facilities and Activities (NZ8) 


\section{2) Move E: Establishing credentials}

It appears in 26 texts in the Thai hotels and 25 texts in the New Zealand hotels. The purpose of this move is to construct positive images of the hotels and to prove to the readers that the hotels are well-established with outstanding and unique services and facilities. Establishing credentials is designed to convince the guests to make a decision to book the hotels. This move thus provides information on different aspects of the hotel such as guest comments, location, scenery of the hotels, awards received, and history. This move can be illustrated by eight steps.

Step dl: Describing the location

Step ih: Informing the readers about the background or history of the hotels and the hosts

Step pc: Providing local knowledge and cultural background

Step cs: Claiming superiority

Step cl: Claiming luxury

Step ia: Introducing the external awards received

Step ig: Introducing the guests' feedback

Step sa: Providing satisfaction assurance

\section{A) Step dl: Describing the location}

This step highlights the outstanding character of location, surrounding scenery, and the tourist attractions nearby. It also informs the readers that the hotel is situated in a good and convenient location for ease of transportation.

Examples:

1) Baan Baitan Resort A Comfortable boutique Resort Thai Style in Rayong with a location that will please the tourist, just minutes from Leam Maphrim Cape, Rayong. Situated between Leam Mae Phrim Cape, Baan Phe Market and Koh Samed. (T3)

2) Visit the real Samui. Elements is nestled in a lovely, isolated, nature marine reserve in the secluded virgin coast on the south west of the island of Koh Samui. Our low rise resort blends naturally into the native 
environment. Uninterrupted views of the tidal lagoon melt seamlessly with our infinity pool and beach area. (T4)

3) Although situated in an oasis of calm, the Black Swan Lakeside Boutique Hotel is only a 5 minute drive from Rotorua City Centre and a 20 minute drive from Rotorua International Airport. (NZ5)

4) The Bolton Hotel has an ideal Wellington city centre location, situated at the junction of the capital's central business district and political heart.

B) Step ih: Informing the readers about the background or history of the hotels or the hosts

The purpose of this step is to provide background and historical information about the hotels or hosts that is often but not always related to their long standing experience in delivering services to the guests. In addition, providing this information suggests that the hotel has a history or is an expert in providing the offers. This step also narrates the impressions and experiences of the hosts.

Examples:

1) The Cape Nidhra Hotel, Hua Hin, is a member of the Small Luxury Hotels of the World and a part of the prestigious Cape Hotel Collection, which includes the famous Cape Panwa Hotel in Phuket. (T5)

2) Villa Royale is a personal creation of renowned artist and architect Mom Luang Tridhosyuth Devakul, better known simply as Mom Tri, who is a descendant of the King Mongkut Rama IV. Mom Tri opened his private summer home eight years ago to provide guests with a truly royal experience. (T6)

3) Our Executive Chef, Sahil Hussein, with over $\mathbf{1 5}$ years experience in reputable Wellington kitchens will meet with you directly to design a menu that suits your requirements, and of course, you can expect only the best produce that NZ has to offer, and true craftsmanship in keeping with ARTISAN's culinary focus. (NZ6) 
4) Hadleigh was built in 2004 by renowned award winning designer Aaron Haddock. His penchant for blending the character reminiscent of older homes into a modern build is well exemplified here. (NZ7)

C) Step pc: Providing local knowledge and cultural background

The purpose of this step is to point out the value or significance of the main local knowledge and cultural background. This step occurs more frequently in Thai hotels (65\%) than New Zealand hotels (35\%). This step emphasises the value of Thai traditional and Buddhists' culture that hotels consider as the main selling point of the hotels while New Zealand hotels focus on the significance of Maori culture and natural beauty.

Examples:

1) The name Bodhi is a partial translation of the Sinhalese word "Bo-gaha" or tree of wisdom because it was the tree under which the Buddha was enlightened. (T7)

2) Ayurveda is a holistic system of healing which was developed among the wise sages of ancient India around 3,500 years ago. It emphasizes establishing and maintaining a balance of the life energies within us, rather than focusing on individual symptoms. (T8)

3) Mãori are the tangata whenua (indigenous people of the land) of New Zealand and their culture is an integral part of New Zealand life. About 15\% of the country's population of 3.8 million is of Māori descent. Māori are a tribal people and their tribes are known as iwi. (NZ 8)

4) The greatest legacy of this legend is the mighty 'lord of the forest' Tāne Mahuta standing victorious in Waipoua forest, with his shoulders still pushed hard against his 'mother earth' and his feet stretched high towards the heavens of his 'sky father'. (NZ8)

D) Step cs: Claiming superiority

This step highlights the unique facilities and services offered by the hotels. Erkutlu and Chafra (2006, p. 286) define boutique hotels as "intimate, usually luxurious or quirky hotel environment". In Boutique hotels, this step is realised by the use of superlative, evaluative adjectives creating positive value of the hotels such as perfect, ideal, top class, exclusive, and unique. 
Examples:

1) Adopting serenity and nature as its main theme, the Bodhi Serene Hotel, an exclusive boutique hotel in Chiang Mai, Thailand, is an architectural masterpiece. The very best of Thai hospitality is stunningly showcased through the designers' creative expressions, style and dedicated attention to details. (T7)

2) An ideal boutique resort for honeymooners, couples, families or simply those seeking to get away from the busy holiday areas. (T4)

3) Awatea Tasman Bay provides the perfect Nelson holiday accommodation base, (NZ3)

4) Our guests rate this as the best breakfast in New Zealand, which sees them through to dinner at night! (NZ9)

E) Step cl: Claiming luxury

This step reflects one of the most important qualities of boutique hotels. It promotes the luxuriousness of the hotels through the use of evaluative adjectives that represent the value of being elegant, expensive, and luxurious.

Examples:

1) The newest bungalow suite deluxe is a luxurious accommodation with a stylish designed interior. (T9)

2) The Pool Villa brings world-class luxury to a world heritage rain forest. (T10)

3) Mollies boutique hotel sets the benchmark for luxury accommodation in Auckland, New Zealand. (NZ10)

4) Delicious food and luxury 5 star accommodation are a Tangley Bed \& Breakfast specialty. (NZ11)

\section{F) Step ia: Introducing the external awards received}

The step presents the external awards, ratings, or popular votes from organisations that the hotels have received. There are some significant differences between the Thai dataset and New Zealand dataset in terms of the way the hotels present 
the information about awards received. The Thai hotels give the list of awards in bullet points while the New Zealand hotels present their award information in a sentence form.

Examples:

1) - Our Awards

- Good Design Award 2013 (Japan)

- Top 25 Small Hotels in Thailand 2013 (TripAdvisor)

- Top 25 Hotels in Asia 2012 (TripAdvisor)

- Top 25 Hotels for Service in Thailand 2012 (TripAdvisor)

- PM Gold Award-Thematic Idea \& Culture (Thailand Boutique Awards 2011)

- Finalist Award Winner in Functional Creations (PM'S Creative Award)

- Travellers' Choice 2011 - Top 10 Hotels for Romance in Asia (TripAdvisor)

- Recommended Design Hotel Award (I-Design Magazine) (T8)

2) Hintok River Camp @ Hellfire Pass is also honored as

One of the top ten adventure resorts 2009 in Asia Pacific

Two Awarded for Thailand Boutique Awards 2011

Top seven gorgeous places to go glamping in 2012 (T11)

3) Kapitea Ridge was named one of the Top Ten Properties of Distinction Award winners for 2005 by New Zealand Travel Inc. (N12)

4) Eliza's has a 4 Star Plus rating from Qualmark, New Zealand tourism's official mark of quality and provides excellent value for money while providing a very high standard of Christchurch accommodation. (NZ9) 


\section{G) Step ig: Introducing the guests' feedback}

This step aims to promote the hotels' creditability through the presentation of guests' comments. The comments are usually presented in quotation marks. Interviews with hotel owners suggests that guests' reviews are important information for the potential guest to consult before making a decision to book a hotel. This is the reason that the hotels present guests' reviews on their hotel websites.

Examples:

1) What People Say:

"Stayed here as the activity part of a family holiday. The history of the area is so rich and so tragic, a living lesson in World War II for the kids and so much more. Lovely camp, pretty luxurious tents and very good food. If you have time take one of the massages on offer - great value and so relaxing!"

2) "Best Restaurant in Samui" Marco Wachter (T12)

3) "Just what two wrung out parents of young children needed... A perfect spot to celebrate our 10th wedding anniversary" - REBECCA \& PHIL, AUCKLAND NZ (NZ2)

4) "Every room features an irresistible panoramic vista and is carefully designed to give privacy to individuals and flexibility for larger groups."

\section{H) Step sa: Providing satisfaction assurance}

This step only occurs in one Thai hotel's website. The purpose is to assure the readers of the best service will be given to satisfy their needs by the hotel. Interview with the hotel owner said "Providing satisfaction assurance" strategy is one of the most important selling points to capture readers' attention. The hotel believes this marketing strategy helps the readers make a decision to book the hotel more quickly. In addition, it is used as a guarantee to prevent guests' disappointment and complaints. If the guests are not happy with the hotel or the hotel does not meet the guests' expectation, the hotel will find a new hotel and provide transportation at the hotel's cost for the guests. However, the interviews from the web readers contradict the belief of the hotel owner. The web readers said that this strategy does not facilitate their decision to book the hotel. The guests' 
decision is based upon the facilities and services offered. If the readers like the hotels from what they see on the website, they will book the hotel.

Examples:

We know that choosing accommodation can be hard. So we are pleased to offer you an assurance that our hotel is every bit as good as we say. Once you arrive at the hotel, if you feel that it is not quite right for you, or that it does not match what is shown on our website, we will gladly refund $100 \%$ of your deposit. (T8)

\section{3) Move DO: Describing the offers}

This move is an obligatory one in the Thai dataset and it occurs in 28 texts out of 30. Even though this is an optional move (22 texts out of 30 ) in the New Zealand dataset, its occurrence is still worth noting because it falls just below the cut-off frequency for obligatory moves. The main function of this move is to inform readers regarding the details about the facilities, services, activities, atmosphere, and decoration presented by the hotels. The hotels have anticipated what kinds of offers that the guests would desire and then the hotels create an emotional appeal and build the image of the offers through the persuasive statements. In addition, this move highlights the major benefits that the guests can expect when visiting the hotels. This move frequently provides information about price and promotions offered by the hotels. This move can be realised by seven steps:

Step da: Describing the atmosphere and the design of the hotels

Step gf: Giving details of facilities/services

Step cd: Catering for individual's desires

Step ie: Inviting guests to enjoy the facilities and services provided by the hotels and explore the surrounding scenery and activities

Step ps: Introducing promotions and specials

Step sc: Offering services and facilities with conditions

Step pe: Providing information about surrounding shops and entertainment

A) Step da: Describing the atmosphere and the design of the hotels 
The purpose of this step is to grab the guests' attention to the design of the hotels by playing to their imagination and emotion through the descriptions of hotels atmosphere and design.

Examples:

1) This boutique hotel in Chiang Mai exemplifies a fusion of Lanna tradition and culture with modern conveniences catering to the needs of today's urbanites. The hotel's graceful gingerbread architecture is accented by hand-carved fretwork and creates a cool and restful environment (T8). 2) Speaking the language of contemporary Thai design, the deluxe rooms, suites and villas are exquisitely appointed with tropical polished wooden floors, bamboo blinds and dark wood furniture, finding the ideal balance between modern calm and exotic luxury in Krabi, Thailand (T15).

3) Now, you can enjoy the classic charm of this historic building the leadlight windows, wood panelling and the magnificent main staircase (NZ9).

4) The décor reflects much of our interests, there is an eclectic mix of art throughout, these include a number of New Zealand works, pencil drawings, etchings, oil and watercolours, and a gallery of Porsche racing has been given space in the study. Guests are welcome to peruse the collection (NZ7).

\section{B) Step gf: Giving details of facilities/services}

This step is the most frequently occurring (85\%) in Move: DO as it advertises what the hotels have to offer. This step presents the information of the facilities and services that the hotels promote. The information presented in this step is often presented in bullet points in the Thai hotel webpages, while in an imperative and affirmative sentence are used in the New Zealand texts.

Examples:

1) - Outdoor swimming pool. Conference room for private meetings or gatherings.

- Library with vast selections of fiction and non-fiction books as well as movies on DVD.

- Laundry and dry cleaning services. 
- Well-equipped Business Center with high-speed Internet access.

- Transportation to Night Bazaar and vicinity.

- Aromatic and traditional Thai massage.

- The Bodhi Restaurant serves both traditional Thai and international dishes.

- The Ficus Bar and poolside bar serves light snacks, alcoholic beverages and cocktails.

- Room service - available 24 hours.

- Parking spaces available.

2) Free shuttle to Hua Hin market for house guests: We provide free shuttle transfer service between resort and Hua Hin market 5 times per day at the following schedule: Leave from resort to Hua Hin market: 11:00 hrs. / 14:00 hrs. / 18:00 hrs. Back from Hua Hin market to resort: 14:30 hrs. / 18:30 hrs. Handicapped Accessibility: (T7)

3) Rates include: Hearty cooked breakfast, complimentary sauna, wireless Internet connections (1000mb), complimentary on-site parking subject to availability, all taxes, a complimentary evening drink and canapés in our lounge between 6-7pm each evening. (NZ14)

4) Relax and unwind at Distinction Queenstown's Spa at Nugget Point, with our spa therapies whilst taking in the views of the surrounding mountain ranges and Shotover River. Afterwards, challenge your companions to a game of tennis or squash on a private court, or enjoy an exquisite dining experience in Birches Restaurant surrounded by awe inspiring views of the rugged Queenstown landscape. (NZ15)

\section{C) Step cd: Catering for individual's desires}

This step provides the description of special offers that hotel aims to satisfy the individual needs of each potential guest. Also, this step attempts to create emotional appeal and involve the readers by focusing on their desire and benefits that the guests can enjoy when visiting the hotels.

Examples:

1) With tailor made wedding services we are happy to arrange all special requests for the happy couple. From large lavish parties with family and 
friends to intimate occasions for two, the efficient staff will ensure your Krabi wedding day is perfect, providing special moments to treasure forever. (NZ16)

2) Under the fine and intuitive touch of professionally trained therapists, not only avail you of their expertise; they attend to your every need and preference. (T14)

3) Seafields tailor-makes everything exactly the way you want it accommodation, pre-ceremony gatherings and pampering, photography and ceremony. (NZ2)

4) Distinction Hotel Queenstown treats each of our customers with the utmost respect and care, and you can expect excellent service, privacy, and the ability to care for your individual needs. (NZ15)

\section{D) Step ie: Inviting guests to enjoy the facilities and services provided by the hotels and} explore the surrounding scenery and activities

This step invites the guests to experience the services, facilities, and activities that the hotels offer. It also encourages the guests to enjoy activities and scenery outside the hotels. Additionally, this step urges the guests to imagine or build a picture of themselves enjoying the facilities and services.

Examples:

1) At Hintok River Camp @ Hellfire Pass, you can be as passive or active as you may like. Some choose to sit back, relax and chill all day long among the fertile nature or in the natural spring water pool. Some choose to go a little bit wild with kayaking in the river, Hellfire Pass trekking, elephant riding, bamboo rafting or even caving in Lawa cave located in Saiyok National Park. (T11)

2) The luscious oasis of serenity, MUTHI MAYA, where you can reconnect with your inner-self once again. Break away from the city's hustles and bustles, where lives are faced with hectic routine day after day. It's now the perfect time to reward yourself a quiet time for reflection; just you, yourself and the beautiful tropical greens of world heritage, Khao Yai. (T9) 3) Explore the attractions the Waikato has to offer, such as the Te Aroha 
Rail Trail or Mystery Creek. Or simply relax and savour the peace and tranquility of our luxury estate, where you will discover style and comfort, all rolled into one. (NZ17)

4) We invite you to get out into the open air and ocean wilderness, to feel the wonders of nature in a truly authentic way. (NZ13)

E) Step ps: Introducing promotions and specials

The purpose of this step is to attract the guests' attention to the promotions and specials that are temporarily offered by the hotels.

Examples:

1) Complimentary Aroma Massage

Book a minimum 7 nights consecutive stay during these dates and get a complimentary 1-hour Aroma Massage in our Spa Royale for each person (T6).

2) Super discount campaign! Enjoy special price cuts From April 05 To May 30, 2014: $10 \%$ on every room reservation in our Hotel! (T16).

3) We offer great accommodation packages as-well with a stay in one of our beautiful Estate Rooms along with seats to the show and the 3 course menu. Costs are from $\$ 350.00$ (based on double/twin share occupancy) or $\$ 220.00$ (single person occupancy) (NZ20).

4) Super Specials for the $2013 / 2014$ season are available (N13).

\section{F) Step sc: Offering the services and the facilities with the conditions}

This step informs the readers about some of the services and facilities come with conditions or certain restrictions.

Examples:

1) As this is not a regular event, and since it is offered only to our guests and their families and friends, we do request that you book your dinner ahead of time. If you wish to book the dinner, please let us know prior to your arrival. (T8) 
2) The Pool Villa are situated some 60 meters above sea level in a serene rainforest and offers unrivalled ocean views \& magnificent sunsets. The Hillside Pool Villas have all steep drop-off from the terraces and infinity pool so parents with young children who wish to book such Villas should be aware that they will have to be especially vigilant in safeguarding their young ones \& toddlers from these potential dangers. The Beach Villas have also a swimming pool with an infinity edge with a $4 / 5$ meters drop off at the end. The pool is NOT fenced in either the Hillside and the Beach villas.

3) As the Cottage is a self-contained, self-catering accommodation space, meals are not included in the rate. However, breakfast and evening meals are available in the Guest House by prior arrangement, and for an additional charge. Please ask your hosts when you check in. (NZ3)

4) Since Gourmet Stay is a very small hotel, the following services are provided upon request

- Baggage Storage

- Daily Newspapers

- Self-service laundry

- Cot available

- Highchair available in café (T21)

G) Step pe: Providing information about surrounding shops and entertainment

This step informs the readers about the shops and entertainment nearby where the readers can explore.

Examples:

1) Chiang Mai has a splendid selection of fine restaurants to serve almost any taste and budget, and we encourage our guests to visit them as well. We are very happy to assist in selecting a suitable restaurant and making bookings for our guests. (T8)

2) Our Nearby Most Famous Activities if you looking for Thai cooking Class, Here is the best thai cooking school, locate on chaweng beach next to our hotel. They open since 1999 till now. Many of visitor come from all over 
the world who want to know the secret about Thai Food must visit here. (T16)

3) If you are thinking of self-catering, you'll be glad to know that there are two good sized supermarkets in nearby Motueka:

Countdown www.countdown.co.nz - at 108 High Street is open from 7am to 9pm, and New World www.newworld.co.nz - at 271 High Street is open from 7.30am to 9pm. Note that both stores are closed on Good Friday, Easter Sunday, and Christmas Day. There are also lots of dining out and take-away options for your evening meals - see our Motueka Cafes \& Restaurants links for some suggestions. (NZ3)

4) Quality local restaurants are only a two minute, hill-free stroll along the seafront and we recommend the best for you. (NZ19)

\section{5) Move: Calling for action (C)}

The most important aspect of this move is to urge some actions from the readers such as making a booking, enquiring for more information, and engaging in further communication. This move has no steps and can be realised in many sentence structures such as imperative and interrogative strctures. This move is elucidated by the following examples:

1) Read more about the Bodhi Serene Hotel a boutique hotel in Chiang Mai, Thailand. (T7)

2) Simply click above to find out more or contact us and ask directly, we will be happy to hear from you. (T4)

3) Read more about our Taupo motel facilities here. (NZ22)

4) Check out our accommodation facilities here. (NZ3)

In order to illustrate the hotel online advertising in the framework of move analysis, the move identification of a sample drawn from each of the Thai and New Zealand datasets is exemplified in Figures 18-23 and Tables 8-13. However, the analysis does not include visual images. 


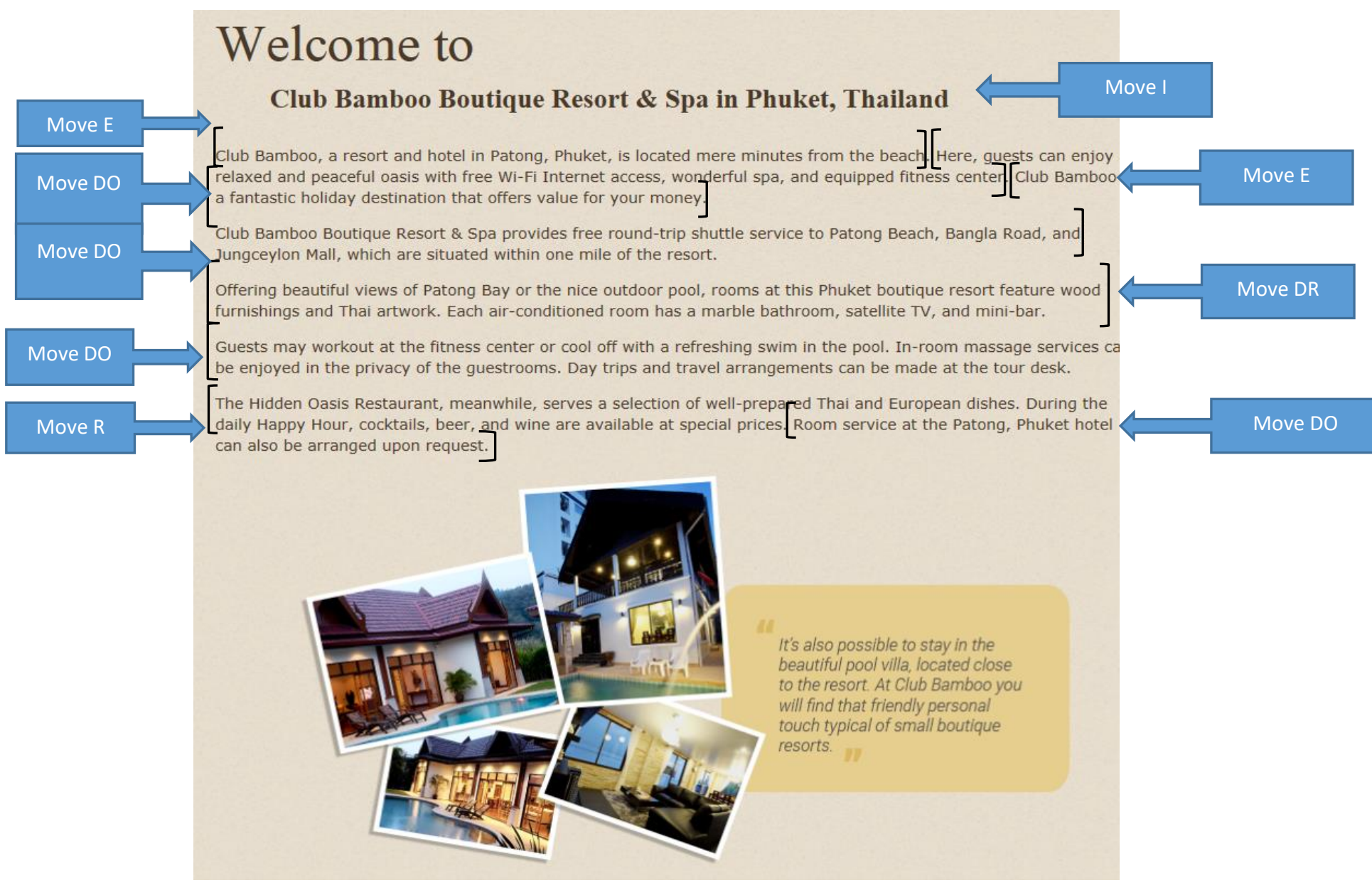

Figure 18: Move identification of Thai hotel Homepage (T10)

Table 8: Move identification of Thai hotel Homepage (T10)

\begin{tabular}{|l|r|}
\hline \multicolumn{1}{|c|}{ Texts } & \multicolumn{1}{|c|}{ Moves/steps } \\
\hline $\begin{array}{l}\text { Welcome to Club Bamboo Resort and Spa } \\
\text { in Phuket, Thailand }\end{array}$ & $\begin{array}{r}\text { Move: Introducing the hotel/facilities/services (I) } \\
\text { Step gr: greeting/welcoming the readers }\end{array}$ \\
\hline $\begin{array}{l}\text { Club Bamboo, a resort and hotel in } \\
\text { Patong, Phuket, is located mere minutes } \\
\text { from the beach }\end{array}$ & $\begin{array}{r}\text { Move: Establishing credentials (E) } \\
\text { Step dl: describing the location }\end{array}$ \\
\hline $\begin{array}{l}\text { Here, guests can enjoy a relaxed and } \\
\text { peaceful oasis with free Wi-Fi Internet } \\
\text { access, wonderful spa, and equipped } \\
\text { fitness center }\end{array}$ & $\begin{array}{r}\text { Move: Describing the offers (DO) } \\
\text { Step gf: giving details of facilities/services }\end{array}$ \\
\hline $\begin{array}{l}\text { Club Bamboo is a fantastic holiday } \\
\text { destination that offers value for your } \\
\text { money }\end{array}$ & $\begin{array}{l}\text { Move: Establishing credentials (E) } \\
\text { Step cs: claiming superiority }\end{array}$ \\
\hline $\begin{array}{l}\text { Club Bamboo Boutique Resort \& Spa } \\
\text { provides free round-trip shuttle service to } \\
\text { Patong Beach, Bangla Road, and } \\
\text { Jungceylon Mall, which are situated within } \\
\text { one mile of the resort }\end{array}$ & $\begin{array}{r}\text { Move: Describing the offers (DO) } \\
\text { Step gf: giving details of facilities/services }\end{array}$ \\
\hline
\end{tabular}




\begin{tabular}{|l|c|}
\hline $\begin{array}{l}\text { Offering beautiful views of Patong Bay or } \\
\text { the nice outdoor pool, }\end{array}$ & $\begin{array}{r}\text { Move: Describing the rooms (DR) } \\
\text { Step df: describing the rooms facilities/ the } \\
\text { outdoor area and the view of the rooms }\end{array}$ \\
$\begin{array}{l}\text { Step ar: describing the atmosphere and the } \\
\text { rooms at this Phuket boutique resort } \\
\text { feature wood furnishings and Thai } \\
\text { artwork. }\end{array}$ & $\begin{array}{l}\text { Step df: describing the rooms facilities/ the } \\
\text { outdoor area and the view of the rooms }\end{array}$ \\
$\begin{array}{l}\text { Each air-conditioned room has a marble } \\
\text { bathroom, satellite TV, and mini-bar. }\end{array}$ & \begin{tabular}{l} 
Step gf: giving details of facilities/services \\
\hline $\begin{array}{l}\text { or coosts may workout at the fitness center } \\
\text { pool. In-room massage services can be } \\
\text { enjoyed in the privacy of the guestrooms. } \\
\text { Day trips and travel arrangements can be } \\
\text { made at the tour desk. }\end{array}$
\end{tabular} \\
\hline $\begin{array}{l}\text { The Hidden Oasis Restaurant, meanwhile, } \\
\text { serves a selection of well-prepared Thai } \\
\text { and European dishes. During the daily } \\
\text { Happy Hour, cocktails, beer, and wine are } \\
\text { available at special prices. }\end{array}$ & $\begin{array}{r}\text { Move: Introducing restaurants/bars, and lounges (R) } \\
\text { Step fr: specifying facilities and services of the } \\
\text { restaurants, bars, lounges, and libraries }\end{array}$ \\
\hline $\begin{array}{l}\text { Room service at the Patong, Phuket hotel } \\
\text { can also be arranged upon request. }\end{array}$ & $\begin{array}{r}\text { Move DO: Describing the offers (DO) } \\
\text { Step sc: offering services and facilities with } \\
\text { conditions }\end{array}$ \\
\hline
\end{tabular}




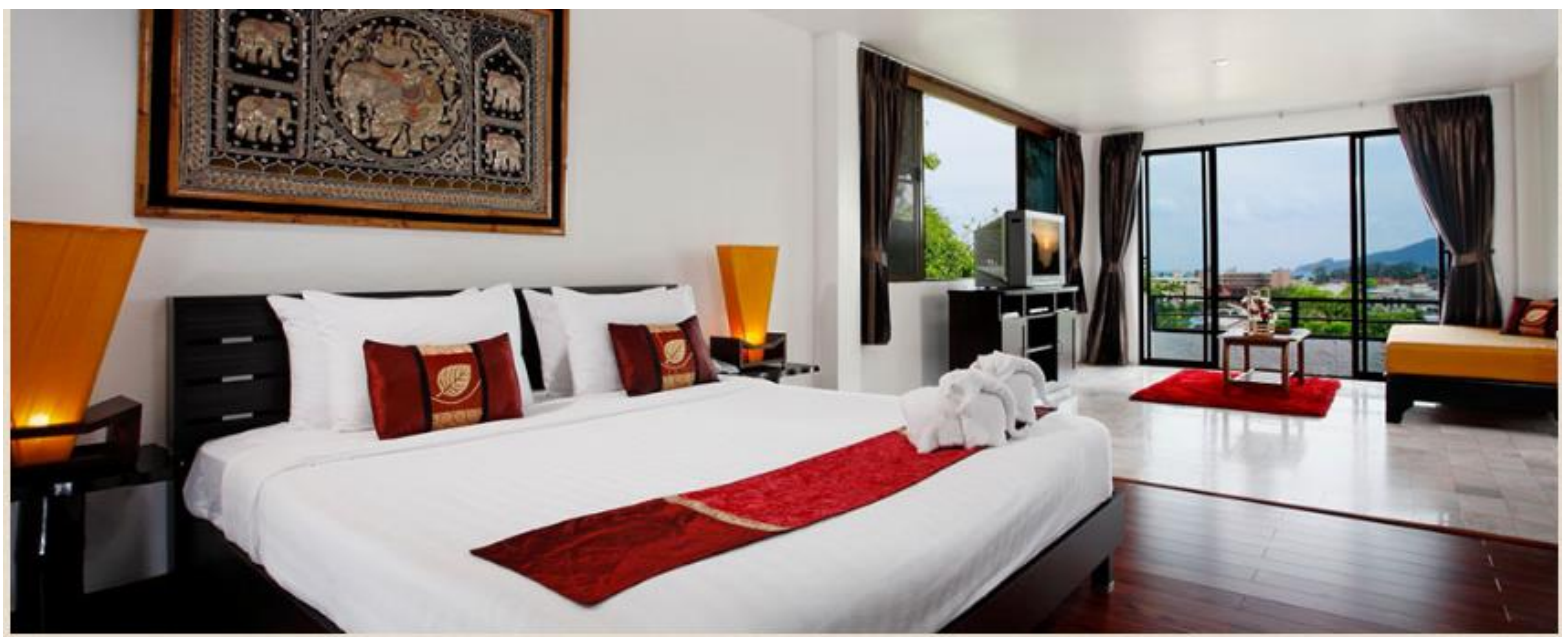

PENTHOUSE SUITE (65 SQM.)

Move I

The elegant and spacious penthouse suite has a beautiful view of Patong and Patong Bay. Our luxurious penthouse suites are decorated in Thai style and are designed to provide our guests the most comfortable stay with a king-size bed, kitchenette and dining table, spacious living area and a large marble bathroom with a king-size Jacuzzi.

In-Room Facilities:

- Air-conditioning

- Flash screen TV

- Refrigerator with Full set of minibar

- Shower with bathtub

- Daily complimentary Tea and Coffee set

- Telephone

- In-room safety box

- Cable TV with international channels

- Hairdryer (on request)

BOOK NOW

Figure 19: Move Identification of Thai Suite page (T10) 
Table 9: Move Identification of Thai Suite page (T10)

\begin{tabular}{|l|l|}
\hline \multicolumn{1}{|c|}{ Texts } & \multicolumn{1}{c|}{ Moves/steps } \\
\hline Penthouse Suite (65 Sqm.) & $\begin{array}{l}\text { Move: Introducing the hotel/facilities/services (I) } \\
\text { Step ph: providing a heading for different } \\
\text { aspects of the hotels }\end{array}$ \\
\hline $\begin{array}{l}\text { The elegant and spacious penthouse suite } \\
\text { has a beautiful view of Patong and Patong } \\
\text { Bay. }\end{array}$ & $\begin{array}{l}\text { Move: Describing the rooms (DR) } \\
\text { Step df: describing the rooms facilities/ the } \\
\text { Our luxurious penthouse suites are } \\
\text { decorated in Thai style }\end{array}$ \\
$\begin{array}{l}\text { and are designed to provide our guests } \\
\text { the most comfortable stay with a king-size } \\
\text { bed, kitchenette and dining table, } \\
\text { spacious living area and a large marble } \\
\text { bathroom with a king-size Jacuzzi. } \\
\text { In-Room Facilities: }\end{array}$ & $\begin{array}{l}\text { Step ar: describing the atmosphere and the } \\
\text { design of the rooms (theme of the hotel } \\
\text { suites) }\end{array}$ \\
-Air-conditioning & $\begin{array}{l}\text { Step df: describing the rooms facilities/ the } \\
\text { outdoor area and the view of the rooms }\end{array}$ \\
$\bullet$ Flash screen TV & \\
$\bullet$ Refrigerator with Full set of minibar & \\
$\bullet$ - Shower with bathtub & \\
\hline - Daily complimentary Tea and Coffee set & \\
\hline Book now & \\
\hline
\end{tabular}




\section{Facilities \& Services}

Move $\quad$ Club Bamboo offers a beautiful swimming pool with a waterfall that flows into the pool water. It is interspersed by drinks and dishes at the pool too. At the restaurant you can eat Thai and European dishes. In the open lobby you have an overlooking of Patong and the surrounding hillsides]

Figure 20: Move identification of Thai hotel facilities and Activities page (T10)

Table 10: Move identification of Thai hotel facilities and Activities page (T10)

\begin{tabular}{|l|c|}
\hline \multicolumn{1}{|c|}{ Texts } & \multicolumn{1}{c|}{ Moves/steps } \\
\hline Facilities \& Services & $\begin{array}{c}\text { Move: Introducing the hotel/facilities/services (I) } \\
\text { Step ph: providing a heading for different } \\
\text { aspects of the hotels }\end{array}$ \\
\hline $\begin{array}{l}\text { Club Bamboo offers a beautiful swimming } \\
\text { pool with a waterfall that flows into the } \\
\text { pool water. It is interspersed by tropical } \\
\text { green, together with a sundeck area with } \\
\text { sun beds. }\end{array}$ & $\begin{array}{c}\text { Move: Describing the offer (DO) } \\
\text { Step gf: giving details of facilities/services }\end{array}$ \\
\hline $\begin{array}{l}\text { The restaurant is located next to the pool } \\
\text { and serves drinks and dishes at the pool } \\
\text { too. At the restaurant you can eat Thai } \\
\text { and European dishes. }\end{array}$ & $\begin{array}{c}\text { Move: Introducing restaurants/bars, and lounges } \\
\text { (R) } \\
\text { Step fr: specifying facilities and services of } \\
\text { the restaurants, bars, lounges, and } \\
\text { libraries }\end{array}$ \\
$\begin{array}{l}\text { In the open lobby you have an overlooking } \\
\text { of Patong and the surrounding hillsides. }\end{array}$ & $\begin{array}{l}\text { Step ds: describing design, exterior } \\
\text { scenery, and atmosphere of the } \\
\text { restaurants, bars, lounges, and libraries }\end{array}$ \\
\hline $\begin{array}{l}\text { If you bring your own laptop, some room } \\
\text { types are equipped with telephone plugs } \\
\text { to access the internet. There are also } \\
\text { direct lines to international phone systems } \\
\text { as well as fax and copying services; } \\
\text { just contact our front desk staff for any } \\
\text { type of requirement which you may have. }\end{array}$ & $\begin{array}{r}\text { Move: Describing the rooms (DR) } \\
\text { Step dfb: describing the rooms facilities/ } \\
\text { the outdoor area and the view of the } \\
\text { rooms }\end{array}$ \\
\hline
\end{tabular}


The Thai boutique hotel webpages consist of six moves, four of them are obligatory moves, and the other two are optional: Move: Calling for action (C) and Move: Introducing restaurants/bars, and lounges (R). The Home page starts with the name of the hotel and its location to attract potential guests who are looking for a hotel in Phuket. Move: Introducing the hotel/facilities/services (I) serves as an introduction to the readers because it gives details on the hotel's name and location. Then the web designer uses Move: Establishing credentials $(\mathrm{E})$ to convince the readers that the hotels are well-established with outstanding and unique services and facilities in order to encourage guests to book the hotel. Since the communicative purposes of moves can be realised through a variety of steps, the web designer uses step: describing the location and claiming superiority to construct their credibility. After that, the hotel uses Move: Describing the offer (DO) to present facilities and services that the hotel has to offer. As move structures are recursive in nature, there are also cases where moves are extensively recycled. The moves that are often recycled through three main webpages are Moves: (DO) and (E). The cyclical process of moves is used as a technique to promote the hotels' offers. That is to say, after introducing and describing facilities and services to the audience, the hotel uses Move $(E)$ to construct and strengthen their positive image and advantages in order to reassure the audience that their hotel is an expert in providing services and facilities.

On Suite page, only three moves are used to advertise the hotel's accommodation. There are Moves (I), (DR), and (C). The presentation of this webpage is quite straightforward. In regard to, Facilities and Activities page, the main focus is to describe facilities and services in the hotel, accommodation, and restaurant. As a result, five moves (Moves: (I), (DO), (R), (DR), and (C)) are used to achieve the main communicative purposes of this page. The next section is the example of how the New Zealand hotel in my dataset exploits move structures to promote its hotel. 


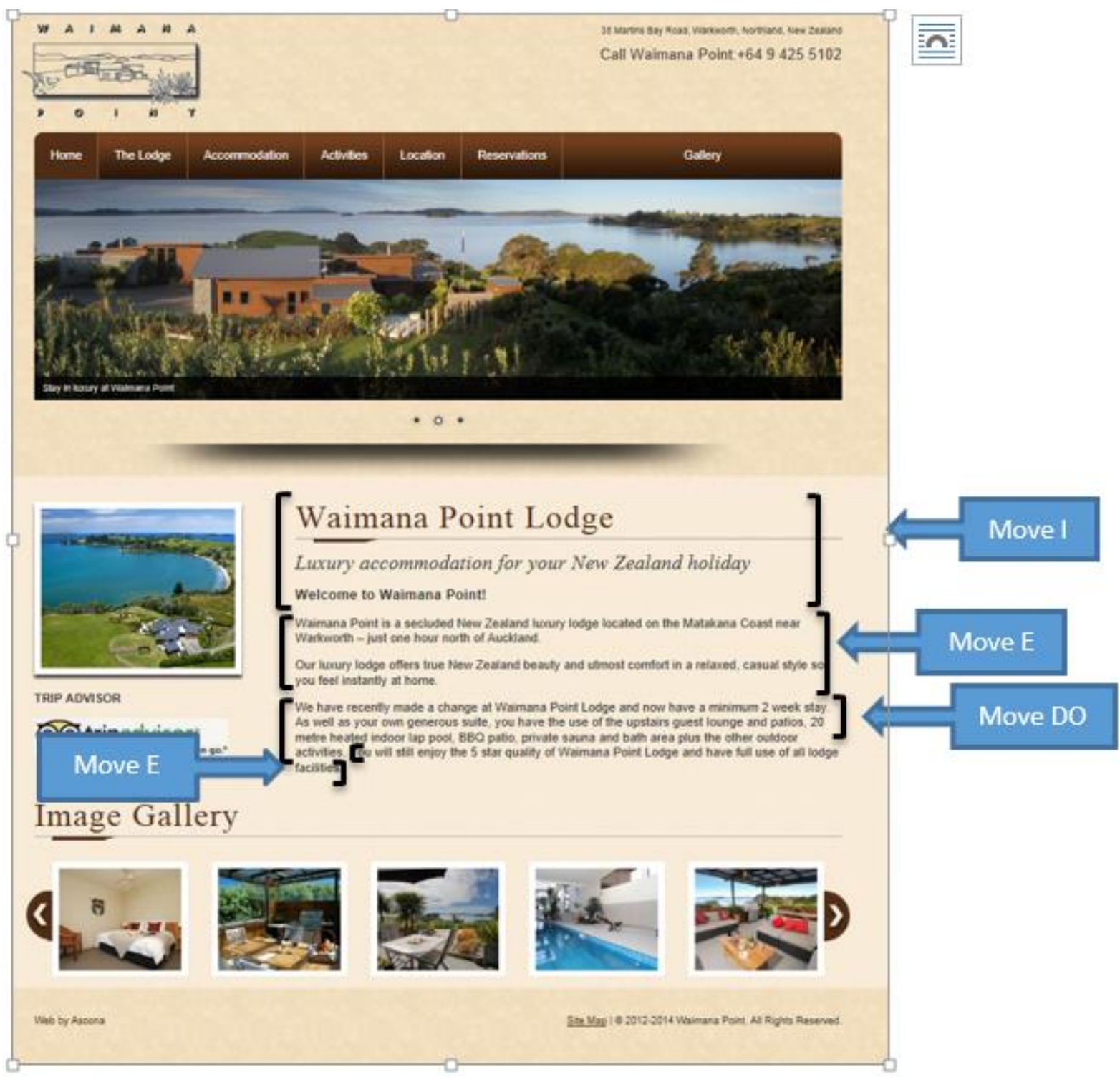

Figure 21: Move identification of New Zealand Homepage (NZ25) 
Table 11: Move identification of New Zealand Homepage (NZ25)

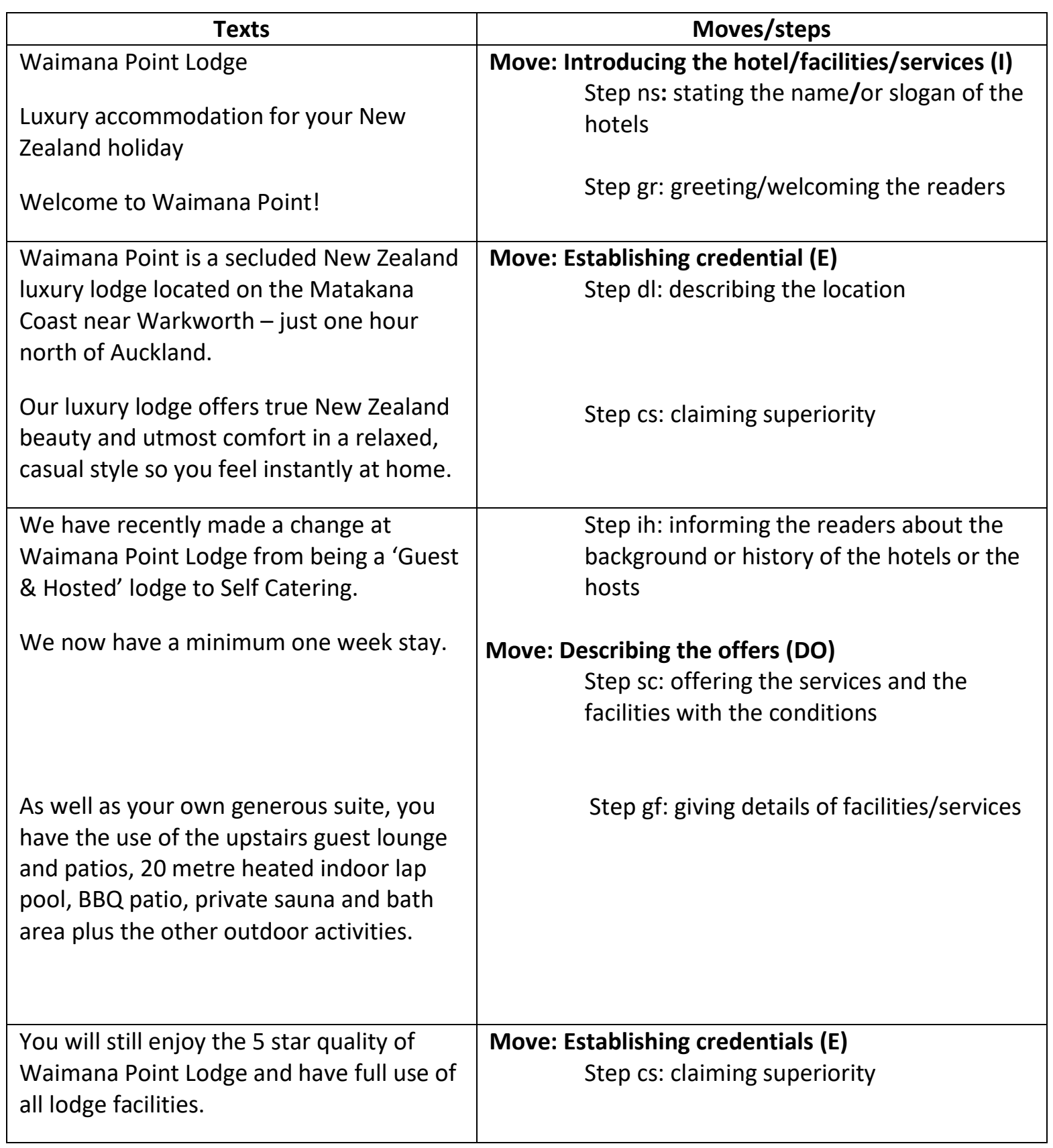




\section{Stay at Waimana Point}

\section{Select from our luxury accommodation options}

We have designed our Matakana luxury lodge for your comfort, and you'll find that our 5 star accommodation has a uniquely New Zealand feel. Our four guest suites are each 50 sq metres and named after native New Zealand tree species. Choose from:

- Kowhai Suite

- Puriri Suite

- Nikau Suite

- Pohutukawa Suite (includes a second bedroom)

Each beautifully appointed guest suite features:

- Super-king beds with fine linen

- Kitchen area with everything you need for a self-contained stay, including a dishwasher, fridge, microwave and bench-top cooking

- Dining area

- En-suite bathroom

- Open plan design with comfortable lounge area

- LCD television, DVD and stereo sound system

- Under-floor heating and ceiling fan

- Complimentary wi-fi and writing desk

- Laundry facilities available

- Suites fully serviced twice a week

Our suites are located on the lower level of the main Lodge building, and each room enjoys stunning sea and rural views. Your private patio area leads from the suite into the garden and to walkways on the property.

The pace of life is leisurely at Waimana Point - so relax in the privacy of your suite and admire the view, savour a glass of fine local wine [take a dip in our 20-metre indoor pool, or lie back with a book from the library]. [it's all here for you to enjoy amongst the peace and tranquility of our luxury Matakana lodge.]

We are happy to discuss the range of activities available in more detail, or answer any questions you have about our eco lodge.

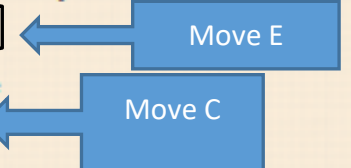

Figure 22: Move identification of New Zealand suite page (NZ25) 
Table 12: Move identification of New Zealand suite page (NZ25)

\begin{tabular}{|c|c|}
\hline Texts & Moves/steps \\
\hline Stay at Waimana Point & $\begin{array}{l}\text { Move: Introducing the hotel/facilities/services (I) } \\
\text { Step ns: stating the name/or slogan of the } \\
\text { hotels }\end{array}$ \\
\hline $\begin{array}{l}\text { Select from our luxury accommodation } \\
\text { options }\end{array}$ & $\begin{array}{l}\text { Step ph: providing a heading for different } \\
\text { aspects of the hotels }\end{array}$ \\
\hline $\begin{array}{l}\text { We have designed our Matakana luxury } \\
\text { lodge for your comfort, and you'll find that } \\
\text { our } 5 \text { star accommodation has a uniquely } \\
\text { New Zealand feel. }\end{array}$ & $\begin{array}{l}\text { Move: Establishing credentials (E) } \\
\text { Step cs: claiming superiority }\end{array}$ \\
\hline $\begin{array}{l}\text { Our four guest suites are each 50sq metres } \\
\text { and named after native New Zealand tree } \\
\text { species. Choose from: Kowhai Suite, Puriri } \\
\text { Suite, Nikau Suite, Pohutukawa Suite } \\
\text { (includes a second bedroom) } \\
\text { Each beautifully appointed guest suite } \\
\text { features: } \\
\text { Super-king beds with fine linen } \\
\text { Kitchen area with everything you need for a } \\
\text { self-contained stay, including a dishwasher, } \\
\text { fridge, microwave and bench-top cooking } \\
\text { Dining area } \\
\text { En-suite bathroom } \\
\text { Open plan design with comfortable lounge } \\
\text { area LCD television, DVD and stereo sound } \\
\text { system Under-floor heating and ceiling fan } \\
\text { Complimentary wi-fi and writing desk }\end{array}$ & $\begin{array}{l}\text { Move: Describing the rooms (DR) } \\
\text { Step df: describing the rooms facilities/ the } \\
\text { outdoor area and the view of the rooms }\end{array}$ \\
\hline $\begin{array}{l}\text { Laundry facilities available Suites fully } \\
\text { serviced twice a week }\end{array}$ & $\begin{array}{l}\text { Move: Describing the offers (DO) } \\
\text { Step gf: giving details of facilities/services }\end{array}$ \\
\hline $\begin{array}{l}\text { Our suites are located on the lower level of } \\
\text { the main Lodge building, and each room } \\
\text { enjoys stunning sea and rural views. Your } \\
\text { private patio area leads from the suite into } \\
\text { the garden and to walkways on the property. }\end{array}$ & $\begin{array}{l}\text { Move: Describing the rooms (DR) } \\
\text { Step df: describing the rooms facilities/ the } \\
\text { outdoor area and the view of the rooms }\end{array}$ \\
\hline $\begin{array}{l}\text { The pace of life is leisurely at Waimana Point } \\
\text { - so relax in the privacy of your suite and } \\
\text { admire the view, savour a glass of fine local } \\
\text { wine, }\end{array}$ & $\begin{array}{l}\text { Step ar: describing the atmosphere and the } \\
\text { design of the rooms }\end{array}$ \\
\hline
\end{tabular}


We are happy to discuss the range of activities available in more detail, or answer any questions you have about our eco lodge.

Move: Calling for action (C)

\section{Waimana Point - Activities}

Make the most of your stay at our luxury lodge with these activities

There's plenty to do while staying at Waimana Point - we have a number of fun and relaxing activities on-site, or you can use your New Zealand holiday time to go off and explore local attractions around the Matakana area.
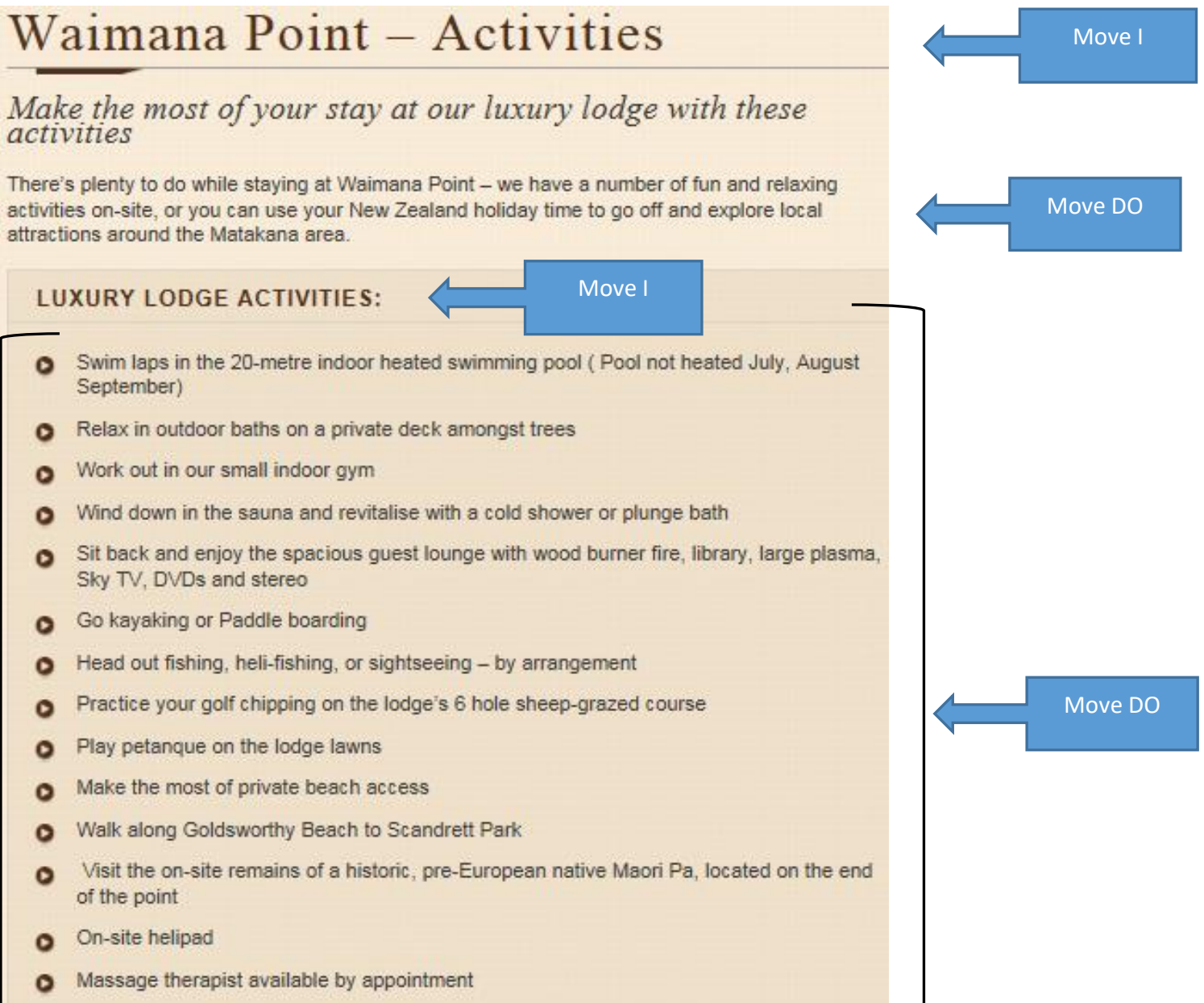

\section{EXPLORE THE MATAKANA COAST:}

\section{Move I}

- Visit the many Matakana Coast vineyards and wine trails

- Play golf at one of the two nearby courses - Warkworth and Omaha

- Browse the Matakana Farmer's Market (Saturday mornings)

- See a movie at the boutique Matakana Cinemas

- Check out local arts and crafts - Morris + James Pottery, and the Brick Bay Sculpture Trail

- Try out local cuisine

- Visit Goat Island marine reserve

- Go horse riding at Pakiri Beach

- Take a water taxi to Kawau Island and see the Historic Mansion House

- Have a surf at Tawharanui or Omaha beach

- Explore local Regional Parks - Scandrett (only half an hour's walk from the lodge), Tawharanui, Mahurangi, and other local walking trails. 
Want some personal recommendations? Just have a chat with your hosts Geoff and Gloria - they'll be happy to recommend other activities you may enjoy. Or visit the Matakana Information Centre to find out more about what's on in Matakana.

Figure 23 Move identification of New Zealand hotel facilities and Activities page (NZ25)

Table 13: Move identification of New Zealand hotel facilities and Activities page (NZ25)

\begin{tabular}{|c|c|}
\hline Texts & Moves/steps \\
\hline $\begin{array}{l}\text { Waimana Point } \\
\text { - Activities Make the most of your stay at } \\
\text { our luxury lodge with these activities }\end{array}$ & $\begin{array}{l}\text { Move: Introducing the hotel/facilities/services (I) } \\
\text { Step ns: stating the name/or slogan of the } \\
\text { hotels } \\
\text { Step ph: providing a heading for different } \\
\text { aspects of the hotels }\end{array}$ \\
\hline $\begin{array}{l}\text { There's plenty to do while staying at } \\
\text { Waimana Point - we have a number of fun } \\
\text { and relaxing activities on-site, } \\
\text { or you can use your New Zealand holiday } \\
\text { time to go off and explore local attractions } \\
\text { around the Matakana area. }\end{array}$ & $\begin{array}{l}\text { Move: Describing the offers (DO) } \\
\text { Step gf: giving details of facilities/services } \\
\text { Step ie: inviting guests to enjoy the facilities } \\
\text { and services provided by the hotels and } \\
\text { explore the surrounding scenery and } \\
\text { activities }\end{array}$ \\
\hline Luxury Lodge Activities: & $\begin{array}{l}\text { Move: Introducing the hotel/facilities/services (I) } \\
\text { Step ph: providing a heading for different } \\
\text { aspects of the hotels }\end{array}$ \\
\hline $\begin{array}{l}\text { Swim laps in the 20-metre indoor heated } \\
\text { swimming pool (Pool not heated July, } \\
\text { August September) } \\
\text { Relax in outdoor baths on a private deck } \\
\text { amongst trees } \\
\text { Work out in our small indoor gym } \\
\text { Wind down in the sauna and revitalise with } \\
\text { a cold shower or plunge bath } \\
\text { Sit back and enjoy the spacious guest } \\
\text { lounge with wood burner fire, library, large } \\
\text { plasma, Sky TV, DVDs and stereo } \\
\text { Go kayaking or Paddle boarding } \\
\text { Head out fishing, heli-fishing, or } \\
\text { sightseeing - by arrangement } \\
\text { Practice your golf chipping on the lodge's } 6 \\
\text { hole sheep-grazed course }\end{array}$ & $\begin{array}{l}\text { Move: Describing the offers (DO) } \\
\text { Step gf: giving details of facilities/services }\end{array}$ \\
\hline
\end{tabular}


Play petanque on the lodge lawns

Make the most of private beach access Walk along Goldsworthy Beach to Scandrett Park Visit the on-site remains of a historic, pre-European native Maori Pa, located on the end of the point

On-site helipad

Massage therapist available by appointment

Explore the Matakana Coast:

Visit the many Matakana Coast vineyards and wine trails

Play golf at one of the two nearby courses

- Warkworth and Omaha

Browse the Matakana Farmer's Market (Saturday mornings)

See a movie at the boutique Matakana Cinemas

Check out local arts and crafts - Morris + James Pottery, and the Brick Bay Sculpture Trail

Try out local cuisine

Visit Goat Island marine reserve

Want some personal recommendations? Just have a chat with your hosts Geoff and Gloria - they'll be happy to recommend other activities you may enjoy. Or visit the Matakana Information Centre to find out more about what's on in Matakana
Step ie: inviting guests to enjoy the facilities and services provided by the hotels and explore the surrounding scenery and activities

Step gf: giving details of facilities/services

Step ie: inviting guests to enjoy the facilities and services provided by the hotels and explore the surrounding scenery and activities
Move C: Calling for action

The New Zealand boutique hotel webpages (Figures 21-23) contain five moves: four of them are obligatory, and the fifth is optional (Move: Describing offer (DO)). The hotel begins their three main webpages with Move: (I) to introduce the hotel's name, welcome the readers, and introduce facilities and services. This move is usually located at the top of the page and the beginning of the subsections. Similar to the Thai hotel webpages above, the move recycling technique is also used between Moves: (DO) and (E), particularly on the Home and Suite page in New Zealand hotel. In addition to these two moves that are recycled, Move: (I) and Move: (DO) also recur. At the bottom of Suite and Facilities page, after introducing and describing facilities and services to the audience, 
the hotel uses Move: (C) to urge some actions from the readers. In this context, the hotel hosts encourages the readers to engage in further communication with the hosts and find out more information.

\subsection{Conclusion}

The move analysis carried out of the Thai and New Zealand webpages shows that the move structures are dynamic rather than static, and the communicative purposes of moves can be fulfilled through the use of a variety of steps. The realisation of moves on the Thai and New Zealand hotel webpages shares some significant similarities. Firstly, three obligatory moves are used in both hotel webpages: Move: Introducing the hotel/facilities/services (I), Move: Establishing credentials (E), and Move: Describing the rooms (DR). The findings show that the cyclicity of moves in both datasets is a common technique used to promote hotel webpages. I speculate that this is because the cultural background of the web designers does not have a major impact on the way they write. In contrast, the conventions of online promotional genres is the most important influence on the regularities in their writing of hotel webpages. 


\section{Chapter 6: REGISTER ANALYSIS}

In the previous chapter, I discussed the identification of moves and steps and their communicative functions in the boutique hotel webpages in the Thai and New Zealand datasets. In order to identify how the web designers use language to influence their target audience, this chapter examines the hotel online advertising texts at a register level (micro textual level) by analysing their distinctive lexico-grammatical features which include imperatives, passive voice, pronouns, and modal verbs.

\subsection{Tourism language}

Tourism language is a specialised discourse as it contains specific linguistic properties and specific syntactic structures (Dann, 1996; Pierini, 2009; Francesconi 2011; Ruiz-Garrido and SaorínIborra, 2013). It persuasively informs the readers of tourist destinations and/or hotels through promotional materials such as tourist guides, brochures, tourism websites, and hotel advertising webpages. For this reason, Dann (1996) claims the persuasive power of tourism language can be used to influence society.

Due to on-going demand from travelers in the hotel and tourism industry, several forms of maketing materials are employed to deliver information about important events and promotional deals to tourists. To serve the communicative functions of the marketing materials, the language used must be persuasive, creative, and unique. The specialised characteristics of promotional materials have been a focus of interest and extensively investigated their features in terms of their frequency of occurrence, and collocations and functions by discourse analysts. The analyses of tourism language are found in the following studies: tourist brochure (Francesconi, 2011), the Brief Tourist Information (Henry and Roseberry, 1996), accommodation websites (Manca, 2008), tourism websites (Maci, 2007; Pierini, 2009), hotel brochures (Mongkholijuck, 2008), tourist brochures (Pitchinee, 1995).

The language found in this tourism genre shares certain linguistic similarities. Pitchinee (1995) and Francesconi (2011) found that personal pronouns such as You, We and Our are employed as a strategy to invite viewers to visit hotel or tourist sites. Imperatives, too, are frequently found in promotional genres (Henry \& Roseberry, 1996; Pitchinee, 1995). Another aspect commonly used to elicit action from the audience involves the modals (Can and Will) (Henry \& Roseberry, 1996; Maci, 2007; Pitchinee, 1995). Extensive use of complex noun phrases is found on hotel and the tourist 
sites, with the intent to create desire in the tourists to visit the hotels and places in the tourists (Mongkholijuck, 2008). Use of metaphors and adjectives to enhance the imagination and mood of readers has also been noted (Pitchinee, 1995). Even though the abovementioned features have been investigated in a variety of contexts in terms of their functions and collocations, few research have studied hotel webpages and none of them have examined these features in the boutique hotel context. Online advertising by boutique hotels is particularly interesting to ESP specialists because the small scale of boutique hotels means that webpages are often produced in-house. In my study, I mainly focus on the use of imperatives, the passive voice, personal pronouns, adjectives, and modal verbs. These features are valuable assets that are used to achieve a particular intention of each move. I would also like to see whether the functions of these features are different from other kinds of advertisement within the same broad set of promotional genres.

\subsection{Register of boutique hotel webpages}

This part of my study sought to investigate the following questions: 1 ) What are the predominant lexico-grammatical features used to serve genre-specific functions? 2) What are the similarities and differences in the use of the prominent lexico-grammatical features between the boutique hotels in Thailand and in New Zealand? And 3) What contextual factors influence the variation in the use of distinctive lexico-grammatical features between boutique hotels in Thailand and in New Zealand?

The linguistic analysis covers five main prominent features: 1) imperatives), 2) the passive voice, 3) personal pronouns, 4) adjectives, and 5) modal verbs. I selected these five features in my analysis because they are frequently occurring words found in previous tourism material studies and also in mydataset (see more detailed discussion of how I selected these features in section 4.3.2 in chapter 4).

To provide data for analysis, I collected data in electronic format taking into account its representativeness of boutique hotel webpages in Thailand and in New Zealand. This datasetrepresents 61,457 words. The frequency of wordlist and the collocations of the distinctive features are generated by AntConc 3.2.4. This section begins with a summary of the distinctive features found in my datasets. Then the similarities and differences between the functions and the frequencies of occurrence of these features in the Thai and New Zealand datasets are discussed. 


\subsubsection{Imperatives}

Imperatives are one of the most distinctive features in the boutique hotel advertising webpages. They occur in every move except for Move: Establishing credentials (E). Even though the imperative syntactic construction is relatively straightforward, they offer a wide range of functions. Aikhenvald (2010, p. 3) explains that imperatives only collocate with a specific sets of subjects and objects, but at the same time they can be used in many circumstances such as invitations, requests, and instructions. According to Swales, Ahmad, Chang, Chavez, Dressen, and Seymour (1998), imperatives have two main surface representations: 1) have no surface subject (apart from occasional uses of You, as in You try this), and 2) have either a main verb or emphatic do in the base form and no modals, as in Do be careful. In this analysis, these two imperative structures are used to achieve the persuasive function in hotel online advertisements. However, there was no evidence of emphatic do structure being used. These two structures are used by web designers to perform a variety of marketing strategies in boutique hotel webpages in the Thai and New Zealand datasets; for example, inviting the readers, engaging the readers, and directing the readers. Similarly, these functions are also found in other advertising discourse e.g. Janoschka (2004), Maci (2007), Myers (1994). Maci (2007) investigates advertising language in British tourism websites. Maci's study reveals that imperatives are one of the most distinctive features that are widely used to invite the readers to perform some actions.

Apart from advertising discourse, imperatives are also commonly adopted in an academic setting in order to achieve a similar persuasive impact. In academic discourse, imperatives possibly can be interpreted as persuasive or manipulative by the readers, depending on the context (Swales, Ahmad, Chang, Chavez, Dressen, \& Seymour 1998; Hyland, 2005). Hyland (2005) points out that imperatives are often used in academic writing to guide and engage the readers. Swales et al. (1998) also state that imperatives are employed by scholarly writers in the ten different academic disciplines that he considered for many reasons, for instance, tools for economy and engaging the readers. The following are the verbs that occur in imperative form in the boutique hotel webpages.

Table 14: The most frequently used imperatives in the Thai and New Zealand datasets

\begin{tabular}{|l|l|}
\hline \multicolumn{1}{|c|}{ Moves } & \multicolumn{1}{c|}{ Verbs } \\
\hline Move: Introducing the hotel/facilities/services (I) & Stay \\
\hline Move: Describing offers (DO) & Allow, Enjoy, Relax \\
\hline Move: Describing rooms (DR) & Enjoy \\
\hline Move: Calling for action (C) & Contact, Book, Read, \\
\hline
\end{tabular}


Table 14 demonstrates seven imperatives across four moves in the boutique hotels in the Thai and New Zealand datasets. According to Brown and Levinson (1987), imperatives can be regarded as bald on record face-threatening acts, which can create a negative feeling or opinion in the hearers. However, in my dataset imperatives occurs very frequently to act as recommendations or invitations rather than commands. For example, Allow and Enjoy are the most frequent imperatives that appear in Move: Describing offer (DO) which is adopted as a persuasive device to promote hotel facilities and services as can be seen in the following patterns:

\section{Table 15: Collocations of allow and enjoy in the imperative form}

\begin{tabular}{ll}
\hline Imperatives & Patterns \\
\hline Allow (39) & - time \\
Enjoy (24) & services and facilities \\
\hline
\end{tabular}

Table 15 shows the syntactic patterns of imperative verbs. Allow collocates with time to signify the amount of time that a spa service would take. An example is "Traditional Thai Massage Allow $\mathbf{1}$ hour or $\mathbf{1}$ hour $\mathbf{3 0}$ minutes or $\mathbf{2}$ hours Thai massage is an ancient massage (T12)." On the other hand, Enjoy co-occurs with a variety of services and facilities that the hotels aim to invite the guests to experience. For example, "enjoy a full manicure (T12)." Maci (2007) points out that imperatives: find, visit, see, and enjoy are one of the most dominant features in her study. Most of them are experiential verbs that are used not only to guide the readers but also to give space and freedom to the readers to make a decision whether they want to visit the destination or not.

As I mentioned earlier, imperatives have versatile roles in my dataset; apart from the above persuasive function of imperatives, a directive function is also important. According to Janoschka (2004) imperatives in web advertising are used to instruct users and initiate an action. They are not employed as offensively as a strict command; they rather perform a polite request and give the readers space to respond. An example is to click here. Hofacker and Murphy $(1998$, p. 1) also suggest that Click here is a call for behaviour kind of imperative, which has a positive impact on promotional texts. Likewise, on the boutique hotel webpages, these instruction verb structures are frequently found in Move: Calling for action (C), where the main purpose is to encourage readers to initiate certain action. Examples are, Contact, Click, Book, Read, and Check out. All these verbs are presented in the imperative, which I consider as the most prevailing linguistic feature in this move (See Table 16). 
Table 16: Collocations of Book, Click, and Check out in the imperative form

\begin{tabular}{ll}
\hline Imperatives & Patterns \\
\hline Contact & -hotel staff members \\
Book & -now \\
Click & -here \\
Check out & -facilities
\end{tabular}

In this analysis, imperatives are used more often in the New Zealand dataset than the Thai daraset.The most frequent verb in the Thai hotel websites in this move is Contact. It usually cooccurs with hotel staff members, as in this example: "Contact our e-concierge for details." The hotel web designers encourage the readers to initiate contact with hotel staff for more information. In contrast, Click is the most frequent verb in the New Zealand dataset. Click commonly collocates with Here to direct a specific location for the readers to browse for more information. In addition, Click is mainly used in the imperative form in a combination with navigational link to encourage the readers to click the links and navigate further to get more information from the websites. Navigational links usually provide a channel for a further contact, downloading menus or brochures, or making a reservation. An example is "Click here to enquire or make a booking at the Spa at Nugget Point."

The next verb is Book, which is encountered in the Thai and New Zealand hotel websites. It frequently co-occurs with Now in both datasets to elicit an immediate response from the audience as in this example: "Book now." This linguistic device is used to provide a reservation channel and encourage the readers to make an instant booking. Web designers use Book now as a marketing strategy to get an immediate response from the readers, right after provision of hotel facilities/services or current promotions.

The last verb that mainly appears in the Thai websites is Read. It often collocates with More with a main purpose to urge the web audience to browse further for more information about details of the rooms or special offers. An example is "Read More." Interestingly, in the New Zealand hotel text, the phrasal verb Check out is frequently used, while it rarely occurs in the Thai dataset. The concordances reveal that there is only one instance of Check out used in the Thai hotels, which indicates it is not a significant linguistic resource that web designers use to achieve the main communicative purpose within Move: (C). In contrast, the New Zealand websites, web designers frequently use Check out, which sounds casual and as if the hotels are interacting in person with the readers to encourage the readers to click the provided links to learn more information. In addition, check out often co-occurs with facilities and services that the hotels offer. For example, "Check out our accommodation facilities here." 


\subsubsection{Use of Passive Voice}

Passive voice is grammatically realised as the reverse of the order of the subject and object of active voice construction (Johnson-Laird, 1968, p. 69). My overall analysis demonstrates that most verbs are represented in the active voice in order to promote hotels. However, there are three moves in particular that use the passive voice. They are Move: Establishing credentials (E), Move: Describing rooms (R), and Move: Describing the room (DR). Passive voice is the main syntactic construction that is used in these three moves in order to promote the location (Move: $E$ and $R$ ) and describe design and decoration of the setting and environment. Very few studies have been conducted to investigate the writers' intention in using the passive voice form, and none of them investigated advertising and tourism purposes.

There are a few discourse studies that investigate the role and function of passive voice. According to Bohner (2001), the passive voice is adopted as an agent avoidance strategy to obscure who is responsible for the action. For example, Bohner (2001) claims that the passive voice is more commonly used than the active voice in sexual violence materials in order to focus on the person who is acted upon rather than the actor of the violent scenes. In Bohner's (2001) study, agentless passive voice seems to be a linguistic indicator of reprehending victims of sexual violence and provoking a negative perception toward the victims. Similarly, Ferreira (1994) indicates that in her experiments, the passive voice form is employed more frequently than active voice in themeexperiencer verbs (verbs where experiencer is the object of an active clause such as surprise and regret Cupples, 2012, p. 125) because the speakers put an emphasis on the subject of the sentences. Furthermore, in investigating the role of the passive in short articles, Johnson-Laird (1968) states that in addition to placing more a prominent role on the subject of the sentences, the passive voice is also used for stylistic purposes. It seems that my study is partially consistent with the above mentioned studies in that web designers attempt to emphasise the subject of a sentence, as the agent is already known by the readers. The focus of the sentence then falls on the new information. My observation is clearly illustrated in Move: Describing rooms (DR) for example, "Each of the rooms are decorated and furnished in authentic Lanna." In this context, the hotels use the passive voice in order to focus on the subject (each of the rooms) and the information follows it. This observation is mainly applicable within Move: (DR). The following are the verbs that occur in the passive voice in the three moves. 
Table 17: Most frequently used passive voices

\begin{tabular}{|l|l|}
\hline \multicolumn{1}{|c|}{ Moves } & \multicolumn{1}{c|}{ Verbs } \\
\hline Move: Establishing credentials (E) & be located \\
\hline Move: Describing rooms (DR) & be equipped, be designed, be decorated \\
\hline Move: Describing restaurant (R) & be located, be served \\
\hline
\end{tabular}

It seems that the hotels use the passive voice to present information as given. The most frequent nouns that are placed as the subject of this structure are mainly hotel names, hotel restaurants, and types of accommodation for example, Le Vimarn cottages and spa, Blue sky restaurant, superior rooms, bungalows, villas, family rooms, and deluxe room. The web designers use this structure in order to attract the readers' attention to the hotel facilities and services that the hotel aims to advertise. For example, when Le Vimarn advertises, the web designers says "Each unit is handsomely decorated in a distinctive island style...." Since the hotel is located on one of the most popular islands, this particular hotel may be aiming to match the design of the hotel accommodation with its location as their main selling point, island theme.

In addition to the above structure, the hotels also use the integration of passive voice and possessive Your to highlight the subject of the sentence and create a personal relationship with the readers. There are two instances of passive combining with personal possessive Your as in "your apartment is equipped with air-conditioning and heat converter...." By employing this integration, the web designers aim to make the sentence more personal and underline the importance of the subject (the apartment) in which the facilities are provided and the privacy of the guest toward the apartment. Your also conveys a personal relationship between the hotel and the guests and highlights the guest's ownership of the apartment, where the guests can enjoy facilities that are offered. It seems as if this apartment is personally and individually offered to You.

According to Hale (2012), the passive voice structure sounds less energetic and impersonal. It is a common construction that is used in legal documents and manuals. However, in the hotel advertising context, I find that the passive voice is a common promotional strategy. Even though the passive voice has sometimes been characterised as conveying lifelessness in tone and mood (Hale, 2012), it is not the hotels' intention to convey that atmosphere to their audience. Rather, the hotels highlightthe subjects (the rooms the hotels aim to promote) by using the passive voice. Moreover, the structure of these verbs in my dataset: Located, Equipped, Designed, and Decorated themselves allows only inanimate subjects. Mostly, types of accommodation are used as the subject of a passive voice structure, as can be seen in the following instances: "the studio is equipped with airconditioning and heat converters for extra comfort." Another example is "These luxurious 
penthouse suites are decorated in Thai style." In these two examples, the studio and these luxurious penthouse are non-living things; they can be therefore placed as the subject of these verbs. The interview data with the hotels in New Zealand does not clarify the reason behind the use of passive voice in the boutique hotel webpages. They explained that they do not know why they use passive voices. They said it was from their general feeling. Similar to the interviews with Thai web designers, they could not comment on why they use passive voice when describing accommodation types. One of the web designers explained that a translation department in his company is responsible for translating Thai webpages into English. Unfortunately, I did not have a chance to interview any of them to gain insights into their practice. However, the appearance of passive voice seems to be a formula or convention that boutique hotels in my datasets use when promoting location and describing the design of the rooms and restaurants.

\subsubsection{Pronouns}

Pronouns are another linguistic strategy that is widely used in advertisements in order to have persuasive impact on the audience. The extent of the impact depends upon how pronouns are used. In addition, contributing to their persuasive effect, they are also used as a tool to construct the speakers' identity (hotels) and the identity of the audience who are the potential guests. Pronouns are grammatically categorised into personal pronouns, possessive pronouns, reciprocal pronouns, reflexive pronouns (Aaron \& McArthur, 1998). However, I will mainly focus on the most frequent pronouns that occur in my datasets: personal pronouns and possessive pronouns. The following section focuses on how pronouns are used for their persuasive impact in advertising discourse.

Samaru-Charles (2001) studied the irregularities of the use of pronouns in magazine advertising. The absence of clear pronoun referent is considered ungrammatical in general discourse but has a persuasive effect in advertising as in "She can make me laugh even when I am mad at her." The pronouns She and Her have no referent; accordingly, this sentence is an anomaly but it gives appeal to the advertisement. Samaru-Charles (2001) maintains that the lack of referent triggers the readers' imagination. The readers have to create a picture of the referent in their mind; as a result, this process makes the advertisement stay in the readers' mind longer than advertisements that have a clear referent. However, the irregular use of pronouns does not exist in the boutique hotel context. This approach may work effectively with slogans or headlines but not with descriptive paragraphs in boutique webpages. 
Rather, the use of pronouns on boutique hotel webpages contributes to the interaction between the hotels and the readers. They are persuasively used as a tool to directly address the readers. In my study, frequent pronouns are We, Our, You, and Your. We and You are personal pronouns and Our and Your are considered as possessive pronouns. These pronouns are found in every obligatory move and they are used to engage readers with the hotel promotional texts. The first singular pronoun, and third party pronouns such as She, He, Her, His, They, and Them, are not found in my dataset. This may be because hotel web advertisement is considered as an interaction between two parties: hotels and target guests; as a result, the hotels aim to focus on the audience's benefit and interest rather than on other people.

The frequent occurrence of plural first and second person pronouns in my datasets appears to be consistent with Janoschka's (2004) findings that the use personal pronouns such as You, Your, we and Our in online advertising is purposively duplicated to be parallel to conversational language. It is employed to be similar to a conversation between the authors and the viewers to emphasise the importance of direct interaction between two parties (p. 139). In addition, the use of direct user addressing tool or personal pronouns are a common persuasive strategy in creating a sense of solidarity and creating involvement with target guests (Francesconi, 2001; Fuertes-Olivera, VelascoSacristán, Arribas-Baño \& Samaniego-Fernández, 2001; Hyland, 2001, 2009; Myers, 1994; Pitchinee, 1995). For example, Fuertes-Olivera et al. (2001) investigate the role of pronouns as personal markers in English slogans and headlines. They found three main functions of pronouns in advertising. The most common function of pronouns is to establish a sense of solidarity with the readers. Secondly the advertisers use pronouns as a tool to help the audience to link the products advertised with cultural icons. The last function of pronouns is to help the target guests to connect the advertising companies or their products with a celebrity whom the companies use to endorse their products. In the context of my study, I only encountered the most common function of personal markers, which is pronouns that are used as a tool to build a relationship with the target audience.

This interpretation of the function of pronouns in my analysis has been confirmed by the interview data that I received from one of the hotel owners in New Zealand. The interview reveals the reason behind the use of pronouns in the boutique hotel webpages. The hotel owner explains that "they truly make the readers feel that they could be an extension of their own property." The hotel uses the pronouns to "make all the guests feel that they are part of the family." The owner further maintains that "pronouns are an important thing to engage the viewer's empathy and create an emotion of warmth and relaxed." That is to say, the hotels in my dataset use pronouns to 
establish a relationship with the target audience and engage them through the advertisement. The following are the frequent pronouns: We, Our, You, and Your, which are which occur in all obligatory moves.

Table 18: Most frequently used pronouns

\begin{tabular}{|l|l|}
\hline \multicolumn{1}{|c|}{ Moves } & \multicolumn{1}{c|}{ Pronouns } \\
\hline Move: Introducing the hotel/facilities/services (I) & You, Your, We, Our \\
\hline Move: Establishing credentials (E) & You, Your, We, Our \\
\hline Move: Describing offers (DO) & You, Your, We, Our \\
\hline Move: Describing rooms (DR) & You, Your, Our \\
\hline Move: Describing restaurant (R) & You, Your, We, Our \\
\hline Move: Calling for action (C) & Our, You, Your, Us \\
\hline
\end{tabular}

Table 18 reveals the frequent pronouns which occur in the Thai and New Zealand boutique hotel datasets. You, Your, We, and Our are the most common pronouns because they exist in almost every move, except for Move: (DO) and Move: (C) in which We is missing. Even though my overall register analysis reveals that almost every move contains a similar set of pronouns, these pronouns particularly have their own functions and collocations that are unique within their own move. In addition, there are some similarities and differences in terms of their roles and collocational behaviour across the boutique hotel webpages in Thailand and New Zealand. The comparison of how these pronouns are employed to promote the hotels between these two cultures in detail will be discussed later in each move. In what follows, I discuss the referents of these four pronouns (You, Your, We, and Our) and their main collocations that occur in the obligatory moves.

As mentioned earlier, these pronouns (You, Your, We, and Our) are used as a persuasive device to engage and interact with the readers; they have a similar set of referents, but their collocational behaviours vary based on the move where these pronouns are employed. In this section, only the most distinctive collocation is selected to be discussed in order to illustrate the overall picture of how these pronouns are employed by the hotels in my datasets in order to promote their facilities and services to the readers. Detailed discussion regarding the collocational patterns that each pronoun exhibits in each move is addressed in section 6.3.

Apart from the investigation of the roles of pronouns that emerge from the frequency of occurrence and their collocations in my datasets, I also investigate the identity of the most frequent pronouns: You and We and their possessive markers. In order to understand the functions of 
pronouns in context, it is crucial to know how boutique hotels establish the position and the identity of their target guests through the use of You and We. This helps the readers to associate the identity of You and We and their functions. Drawing on the interview data with the hotels in Thailand and New Zealand and the collocations of You and We, enables me to provide an overall picture of You and We.

The first pronoun that I would like to discuss is You. According Myers (1994) and Janoschka (2004), You is the common pronoun that occurs in promotional texts because it conveys a personal interaction between the speakers and receivers. Additionally, the referent of You is intentionally ambiguous because You can refer to anyone who reads the advertisement (Myer 19996, p. 79). You in the boutique hotel webpages refers to the potential guests whom the hotels target to promote services and amenities. The analysis reveals that the target customers appear to be a couple and/or family group who would like to experience a unique, exciting, private, relaxed and luxurious lifestyle. This broadly portrayed identity is derived from the context where You is represented in my dataset. Examples of adjectives that co-occur with You are: private, amazed, refreshed, romantic, ultimate comfort, and unique. Based on the frequency of these adjectives, I derive the image of the target guests. This overall picture of You as a target group shares some overlapping qualities of boutique hotel target audience to the previous studies conducted by Covelli (2005) and Lim and Endean (2008). Those studies state that boutique hotel phenomenon has gained popularity among travellers who seek more sophisticated experiences and intimate service in staying at an innovative form of hotels. In addition, Anhar (2001) indicates that apart from expecting to have special experiences from the hotels, the primary purpose of the travellers visiting boutique hotels is to construct their image as a fashionable tourist who can afford to stay in a boutique hotel.

Apparently, in terms of boutique hotel marketing, the interviews with the hotel owners does not truly reveal the characteristics of their target guests. When I interviewed the hotel owners both in Thailand and New Zealand about their target groups, instead of explaining the characteristics of their target groups in terms of touristic experience preference, some of them defined their target group based on geographical aspect. For example, one hotel owner in New Zealand says that their target group is mainly from France and their website has a French language option for the readers to choose. Another similar example is one of the boutique hotels in Thailand. The hotel owner says that their main guest market is from Europe. Furthermore, these two hotels have international agents to seek potential guests for them. Practically, from the interviews, the target guests are recruited based on the market instead of a particular inherent characteristic of the guests. 
Another interesting example is from the interview with the owner of one of the boutique hotels in Bangkok. It shows a mismatch between the hotel's intended promotional aim and the actual use by the travellers. This hotel is located near the main river and the hospital. The hotel is surrounded with old temples and historical sites. Specifically, the hotel is situated in the prime area for guests who would like to see the old town of Bangkok. Apparently, in addition to guests who would like to see the beauty of the river and historical site, the majority of the guests are those whose family members are staying in the hospital. They choose to visit this hotel because of the ease of transportation and convenience, not for the benefit of the river view that the hotel primarily aims to offer.

However, there are only a few hotels in my dataset that have a clear and specific picture of their target market. One of them is a hotel in the North of Thailand, which was built in a historical area. It has a specific image of the guests that they aim to target. Due to its location, the main target group are foreigners who would like to witness the authentic lifestyle of Northern culture and art. The theme of the hotel, staff members' uniforms, and the presentation of food provided also match with the local culture. In an interview with the hotel general manager, she explained that the guests come to the hotel because they would to like to actively engage with local community to experience their culture and life style. They are interested in seeing how food is prepared and decorated, and how local people speak their language. This idea seems to correspond to the value added criterion (local knowledge that the guests can get from the hotel hosts) suggested by McIntosh and Siggs $(2005$, p. 7).

In short, the characteristic of boutique hotel target guest which is represented by You in my dataset seems diversified and dynamic and depends on several factors such as location, marketing strategy, theme and the purpose of the hotels. However, based on the examination of the frequent adjectives that appear near You and interview data, You seems to represent families on vacation and couples on a honeymoon trip who seek to have a luxurious and relaxed lifestyle as well as experience local culture. The following section discusses how You is linguistically adopted in the context of boutique hotels.

In regard to the main collocation of You, the analysis reveals that You frequently collocates with can and will followed by facilities and services that the hotels aim to represent. This may be because of the hotels use of this structure as a linguistic tool to offer the guests an opportunity to experience the hotel facilities and services as in "You can expect excellent service, privacy...." and "You can choose 2 styles of wagon bedrooms...." It seems that the web designers adopt this construction as a technique to describe facilities in order to avoid the routine descriptive statements 
as in "the hotel has.../the hotel provides..." Instead of generally informing the guests what the hotels plan to offer to them, the hotels use can and will as a casual invitation to persuade the guests to book the hotels.

The second pronoun is Your, which refers to the ownership of You as a target guest. A primary intention of using Your by the hotels is to make the readers feel included and that their individual desires are recognised, as in "They attend to your every need and preference. (T15)" The collocations of Your are not uniform but relatively diversified based on the communicative purpose within each move. Even though the reference of Your can refer to an individual or a group of people, the readers will feel as if they are personally and individually offered (Janoschka, 2004, p. 139). Very often the occurrence of Your in my dataset is used to create privacy and personal space that the guests will have when visiting the hotel as in "Your own cooking facilities and spa bath are a bonus." This example uses Your own to emphasise that the hotel values the importance of privacy that the guests would expect when they visit.

The third most common pronoun is We. There are two types of We according to Harwood (2005) and Rounds (1987). They are Inclusive We and Exclusive We. Inclusive We refers to both speakers and the audience while Exclusive We refers to only speakers. There are many discussions regarding the adaptation of Inclusive We and its persuasive roles, specifically in a political context (e.g., Bull \& Fetzer, 2006; Steffens \& Haslam, 2013). Bull and Fetzer (2006) investigated how politicians use the pronouns You and We to avoid answering awkward questions, since these researchers believe that the meaning of the pronouns is ambiguous and the politicians know how to use them to withdraw themselves from these questions. Similarly, Steffens and Haslam (2013) investigated the role of Collective pronouns such as We and $U s$ in official election campaign speeches. Their study claims that there is a significant correlation between successful election candidates and the frequent use of Collective pronouns. Steffens and Haslam (2013) point out that the uses of Inclusive We and Us are associated with leaders' future success because it signals solidarity between the speakers and listeners. However, only a few studies investigated the impact and the function of Exclusive We. For example, Yu (2006) studied the use of pronouns and its implication in news magazines. The study reveals that Exclusive We connotes the authority of the advertisers of the products that they offer and their intent to build a relationship with the readers. A desire of using We to establish the advertisers' position and build a solidarity with the target audience is also found in my study.

In the context of the boutique hotel online advertising, the main referential domain of We is Exclusive we, which refers to boutique hotels, hotel staff members or the hosts who offer benefits as 
well as facilities and services to guests. However, there are a few instances of Exclusive we which represent hotel guests, who wrote a review about their experience with the hotels on the hotel websites. The first reference: We as a boutique hotel is highly more significant than We as a hotel guest because the later referent only occurs in Move: Establishing credentials (E). A discussion of We as hotel guests will be discussed in detail later in Move: (E). The next section focuses on the identity of We as a boutique hotel where the interpretation is derived from interviews with the hotel owners in Thailand and New Zealand.

In terms of hotel identity, interviews with the hotel owners reveal that there are several apparent similarities and differences between two cultures in terms of their size, character and the way they represent themselves as a boutique hotel. Smallness is one of the crucial criteria that is used to determine boutiqueness (Tidtichumrernporn, 2010). According to Hartesvelt (2006), boutique accommodations vary in a number of rooms, but they commonly range from 20 to 150 rooms owing to a desire to maintain a specialised service for guests. The number of rooms in the boutique hotels in the Thai dataset ranges between 19-45 rooms, whereas a scale of hotels in the New Zealand dataset is much smaller. It is between 3-10 rooms. The numbers indicate that the average capacity of boutique hotel accommodation in Thailand is six times larger than in New Zealand hotels.

Size of the hotels is an influential aspect that impacts the characteristics of the hotels in these two countries. Some of the New Zealand hotel owners in my dataset have converted their own residences into a small boutique lodges which can accommodate a small group of guests. The interview data indicates that the hotel owners would like to individually maintain a personalised service and develop a close connection to their guests. Since the scale of the New Zealand hotels is small, often a husband and a wife will be the hosts who provide a service and take care of the guests. The hotels temporarily hire people when they have extra work to do in their properties, e.g., gardening. One of the most luxurious boutique hotel owners in Auckland confirms that he does everything in the hotel including running the hotel and washing dishes. On other hand, in the Thai datasets, because of the larger scale of the hotels, the hotel owners have a full team to run the hotels for them. The owners are mainly involved in policy planning. Other responsibilities are designated to hotel staff members according to departments. Owing to the difference in size between the hotels in the two datasets, the owners have different strategies and management to operate their hotels.

The interviews also affirm that in addition to the difference of scale of the hotels and the way the hotels are operated between the two datasets, their expected turnover and return of 
investment is also different. Half of the hotels in the New Zealand dataset are open for reservation only in the high season and have a specifically preferred type of guests. For example, hotels near Auckland and in Rotorua are opened only in the high season and the hotel near Auckland only accepts long term guests. The New Zealand government aims to decrease seasonal fluctuation in visitor arrival in order to increase the number of tourists and promote New Zealand tourism (The (Tourism Industry Association, Ministry of Tourism \& Tourism New Zealand, 2015). In contrast, the hotels in the Thai dataset are open all year long and take every opportunity to encourage guests to visit the hotels in order to make the best profit for the hotels. It seems that majority of the Thai hotels focus more on profit compared to the New Zealand hotels. The decision of the New Zealand hotels to open hotels based on the season, may be because the hotels know the travelling patterns of the tourists who have New Zealand as their destination. They may know from experience that climate impacts the reservation rate. On the other hand, the weather in Thailand is milder; it is not unreasonable for hotels to expect guests all year. However, it should be stressed that the New Zealand hotels in my interview data represent a small sample which are not necessarily representative in this regard of boutique hotels in New Zealand.

In addition to the difference in hotel scale between these two datasets, another major distinction is tourism trends and campaigns issued by local governments. The Travel and Tourism business is the main contributor to both national economies; in 2015 the travel and tourism sector contributed $\$ 93.8$ billion to Thai economy (Scowsill, 2015), and \$18.6 billion to the New Zealand economy (The Ministry of Tourism, 2015). Both Thai and New Zealand governments are well aware of this fact; as a result, the governments in these two countries have launched a variety of tourism campaigns to support this sector. The Thai government launched a new tourism campaign Thainess - for the years 2015 and 2016, with a particular emphasis on the essence of the Thai way of life, Thai culture and Thai experience because it is believed that these features attract a lot of foreigners' attention (Wuttanavrangkul, 2014). This explains why many Thai boutique hotels in my dataset have been moving towards to cultural tourism. One of the hotel owners explained that the hotel provides many cultural activities which are congruent with the government tourism campaign to boost a number of tourists who are interested in learning and experiencing local culture. These activities include elephant riding and visiting hill tribe villages. Another hotel also provides Thai cooking classes for guests. On the other hand, a similar New Zealand tourism and marketing campaign is the $100 \%$ Pure New Zealand campaign. It promotes an interaction with New Zealanders and New Zealand landscape. This is because New Zealand is famous for its natural attractions and adventure activities. All of these values are compelling features that attract tourists (The Ministry of Tourism, 2015). Differences in tourism trends between Thailand and New Zealand influence the way 
the hotels in these two countries promote themselves and their services/facilities to the outside world.

Even though these two countries manifest some diversity in terms of their size, operational strategies, and tourism trends supported by their governments, both countries share similar marketing strategies. Their main mutual focus is to provide a personalised service to their guests. Providing a personalised service seems to be their ultimate goal, which is considered as a key component of the boutique hotel category (Hartesvelt, 2006; Tidtichumrernporn, 2010). The interviews indicate that in order to maintain a personalised service, the hotels use email to communicate with their guests prior to their visit to enquire about their specific requirements in order to individually tailor the service to their needs.

Another similarity is in regard to architectural design of the hotels. This aspect appears to be a crucial factor as it is the first impression that the guests would notice when looking for boutiqueness or chicness. The hotel owners in the two countries all believe that apart from providing personalised services, a unique design is an important aspect to attract guests, who are seeking distinctive accommodation to satisfy their expectation. There are a few examples of hotels in both datasets who are successful in differentiating their accommodation from other hotels in their neighbourhoods. These hotels have converted their hotels from a historical building and offer different themes in each suite. One of the New Zealand hotels in my dataset bought a 1850s property and transformed it into a luxury boutique hotel. Each room has its own unique theme and design which reflects the historical background of the hotel. Similarly, in the Thai dataset, a small boutique hotel, which is renovated from a $19^{\text {th }}$ Century wooden house, is popular with guests who have a specific desire to experience heritage setting and historic value.

In short, the overall approach that boutique hotel owners use to promote the distinctiveness of their hotels between Thai and New Zealand datasets is similar. A key linguistic means for doing so is in the use of Exclusive We. They present themselves as a boutique hotel with distinctive characteristics who specialise in providing unique service and experience to niche visitors who like to be surprised by architectural designs. The next section focuses on the main collocations of We which functions in claims of uniqueness and boutiqueness.

\subsubsection{Modal verbs}

The modal verbs include Will, Shall, Can, Could, Must, May, Might, Would, and Should. They are generally used with other verbs to express obligation, possibility, and ability (Biber, Johansson, Leech, Conrad, \& Finegan, 1999).This section presents the modal verbs that occur in the boutique 
hotel websites in Thailand and New Zealand. The most frequent modals in my dataset are Will and Can, especially in Move: Describing offers (DO) (see table 19). In my datasets, these modal verbs exist in all obligatory moves except for Move: Introducing facility and services (I). They occur most frequently (Can: 35 and Will: 50 per 1,000 words) in Move: (DO), where their primary purpose is to inform the audience about the hotel facilities and services. In addition, this move highlights the major benefits that the guests can experience/expect when visiting the hotels. These two modal verbs are used as a linguistic tool to describe and highlight the benefits and advantages which the guests will or can experience when staying at the hotels. Stylistically, these two modal verbs appear to be used as way to describe hotel offers in order to enhance a persuasive impact. The following section discusses modal verbs that are significant in my dataset, which are Will and Can.

Table 19: Most frequently used modal verbs and their frequency

\begin{tabular}{|l|c|c|}
\hline \multirow{2}{*}{ Moves } & \multicolumn{2}{c|}{ Modal verbs and its frequency/1,000 words } \\
\cline { 2 - 3 } & will & can \\
\hline Move: Introducing the hotel/facilities/services (I) & - & 0.30 \\
\hline Move: Establishing credentials (E) & 15.00 & 9.10 \\
\hline Move: Describing offers (DO) & 50.00 & 35.00 \\
\hline Move: Describing rooms (DR) & 1.60 & 2.60 \\
\hline Move: Describing restaurant (R) & 1.90 & 4.46 \\
\hline Move: Calling for action (C) & 2.28 & 3.40 \\
\hline
\end{tabular}

One of the most frequent modal verb is Will, which carries the idea of certainty (Biber et al., 1999) prediction (Coates, 1983) directive (Vine, 2004) or willingness (Crystal, 1985; Quirk, Greembaum, Leech \& Svartvik). Will is widely used in promotional discourse as an obligatory promise (Janoschka, 2004; Maci, 2007; Pierini, 2006; Sinaga, 2012). For example, Sinaga (2012) examines the interpersonal strategies of hotel and apartment in magazine advertising. The study reveals that the copywriters use mood types, modal, and pronouns to influence the audience. In terms of modal verbs, Sinaga (2012) found that Will and Can are frequently used in her study. In her study, Will conveys prediction and Can is used to ensure the guests that they will receive the benefit which is advertised in the advertisement. These previous studies agree with my analysis in terms of the function of Will in advertising. The role of Will in promotional genres reveals deviations and variations from the way Will is usually used in academic discourse. The next section presents the functions of Will in my dataset. 
Looking at the occurrence of Will, it is used most frequently in Move: Describing offer in order to persuasively describe facilities and services as in "You will also find postcards, travel CDs, Thai cooking DVDs...." This example occurs in the context where the hotel promotes their Boutique shop. The hotel uses Will as a persuasive tool to promote their products in the shop. You is positioned in an active role, representing the reader who performs the action of find, making this message more dynamic and creating curiosity with respect to what the guests will discover. Interestingly, web designers also use Will in a passive construction in order advertise their hotels. You is placed on the beginning of the sentence in order to focus on You as a guest. For example, "...you will be shown how to prepare several Thai dishes of your choice." In this example Will functions as a promise of the benefit that the guests will get. Similar to the idea of obligatory promise (Pierini, 2006; Janoschka, 2004; Sinaga, 2012; Maci, 2007), Will connotes a commitment that the proposed benefit will be provided when the guests come to the hotel. The role of Will in hotel advertising is relevant to a reduced conditional clause type 1 where will is used to commit the future action to be fulfilled. For example, (if you visit the hotel), "you will be shown how to prepare several Thai dishes of your choice." Here, the conditional clause (if you visit the hotel) is omitted, and only the main clause which shows the offer is presented. The examples above also suggest that Will often collocates with You, who are referred as a target guest and experiential verbs (e.g., find and enjoy) which is consistent to Maci's (2007) study of tourism web text (see Table 22).

Table 20: Modal verbs and their collocations

\begin{tabular}{|l|l|}
\hline Modal verbs & \multicolumn{1}{c|}{ Collocates } \\
\hline Will & you_+ experiential verb + benefits offered by the hotels \\
\hline Can & you_+ experiential verb + benefits offered by the hotels \\
\hline
\end{tabular}

Although Will conveys prediction or willingness as stated in the literature above, in hotel online advertising the function of Will is more versatile. Will seems to be a persuasive device that web designers use to encourage the guests to visit the hotels in order to receive the benefits that the hotels provide. The use of Will is to make promotional messages more persuasive and inviting. Instead of bluntly informing the readers of what the hotels have to offer, the web designers focus on You as a guest who will receive benefits when staying at the hotels. It is not simply a general description of services and facilities, but rather a promise between the hotels and the audience. The hotels guarantee the guests that they will definitely have an opportunity to enjoy the benefits that hotels commit to on their webpages. This function of Will is also congruent with the interview information with an hotel owner in Auckland (N10). He suggested that descriptions of hotel facilities 
and services should be accurate. Additionally, the combination of Will and You directly engages the guests in the hotels offer and enhances an active role of You as a target guests who get a benefit.

Similar to Will that has three different grammatical meanings, Biber et al. (1999) suggest that Can expresses three different meanings: ability, probability, and permission. Even though Can suggests possibility, its underlying implication is willingness that can be further interpreted as a covert imperative (Coates, 1983, p. 98-99). The study of tourism webpages conducted by Maci (2007) also confirms that Can carries the idea of possibility, which can function as an off-record invitation. Audience have the freedom to choose what to do. Additionally, Can is frequently used in conversation more than in other genres (Biber et al., 1999, p. 495). Likewise, boutique hotel webpages are an interaction between hotels and target guests where the hotels try to convince the guests to buy their service.

The contexts where Can is often used is interesting to observe. Similar to Will, Can frequently occurs when the hotels describe facilities and services (most often in Move: Describing offer). It seems to be used as a persuasive device to describe facilities and services. This persuasive function of Can is similar to Will, as I discussed above. However, the role of Can does not sound like a promise committed to by the hotels but rather a suggestion of facilities and services that the guest can experience. For example, "If you would like to have a peaceful evening in your room...you can rent DVD's of all genres at front desk." and "You can enjoy our spectacular restaurant and bar then relax in the swimming pool or in the in-room spa services." These two examples show that Can is adopted as a persuasive tool to suggest the services and a covert invitation to encourage the guests to come to the hotels. It seems that Can gives room and space for the guests to think and react. The hotels only suggest the offers, but the guests have the authority to make a decision. Furthermore, the integration of Can and You brings the guests close to the hotels. It seems to help the hotels to engage the guests' attention as they go through the advertisement. As I said earlier hotel web advertising appears to be an interaction between hotels and target guests, not a monologue or a simple descriptive statement. The hotels seek every possible way to make their offers enticing to the readers.

In regard to the collocational profile of Can, it shares some similarity with Will (see Table 20). The collocations confirm the relation between these two verbs even though they essentially contain different grammatical meanings. From the Table 22, Will and Can mainly collocate with experiential verbs such as experience, enjoy, use, and find. Furthermore, they both co-occur with you to establish a friendly relationship with the guests and highlight the importance of you as a hotel guest as in " $\underline{Y o u}$ will still enjoy the 5 star quality of...." and "...you can enjoy sauna, Jacuzzi, more than twenty treatments." Based on my overall interpretation, Will and Can are rhetorically used as a persuasive 
device to promote their facilities and services. Both modal verbs are not only used as an information giving function but also as a promise of the offers (Will) or a casual invitation (Can).

\subsection{Register analysis of boutique hotel webpages: Comparative analysis}

A comparison of Thai and New Zealand reveals significant variation in the way the hotels in the two cultures promote their facilities and services based on move structures on three main webpages: Home page, Accommodation page, and Facility page. As previously discussed (Chapter 5 section 5.1), move structures are not fixed but dynamic and flexible. They are unlikely to conform to a completely consistent pattern. Some hotels exploit more moves and steps than others to advertise their facilities and services. In addition, moves have a recurring nature; some moves can recur many times within one text. Apart from this move characteristic, each move also achieves its intention through the use of different linguistic features and structures. As online advertising discourse is a specialised discourse, it contains specific linguistic properties and syntactic structures. The language found in these promotional genres shares certain linguistic similarities. For example, Pitchinee (1995) and Maci (2007) found that personal pronouns such as you, your, we, and our and imperatives are frequently employed as a strategy to invite viewers to visit hotels or tourist sites. These two linguistic features are similarly found in my datasets.

The focus of analysis in this section is to investigate distinctive lexico-grammatical features and their collocational behaviour that are used as a linguistic resource to achieve the communicative purposes in each move in boutique hotel websites in Thailand and New Zealand. This section also investigates whether cultural variation between the two countries influences the differences in the way the web designers adopt promotional language to advertise their hotels online. If there are no major differences in the use of linguistic features between the two cultures, it may be assumed that culturally specific features between both countries do not determine the way the promotional language is used. Rather, this language may follow a particular pattern which is determined by the discourse community of this promotional genre. In this section, the linguistic analysis covers three main types of prominent features: verbs, adjectives, and pronouns. The focus of this analysis is to investigate the collocational behaviour of these two aspects that are used as a linguistic resource to achieve the communicative purposes in each move in boutique hotel websites in Thailand and New Zealand. The selection of distinctive features for the analysis is based on a high frequency of tokens that occur in the datasets; that is, the three most frequent words (whose frequency is not lower than 1.5 per thousand words in the datasets) are selected for study. It may seem that 1.5 is based on an 
arbitrariness of the researcher and a practicality of the analysis. However, there are no previous studies suggesting the ideal number for the analysis of linguistic features. I then compared the frequency of the most frequent words in my datasets with the frequency in both written and spoken registers in the British National Corpus (BNC). The selected words reflect the distinctive characteristics of promotional language that are presented in the boutique hotel websites. After that, I used Log-likelihood calculator (http://ucrel.lancs.ac.uk/llwizard.html) to calculate the P value. I used the P value to determine the differences in statistical significance of the most frequent words between my datasets (Thai/New Zealand datasets) and the BNC. The cut-offs of P value is between $P$ $>0.10$ (less significant)- $P<0.0001$ (most significant). In order to compare the differences in statististical significance of the most frequent words between Thai and New Zealand datasets, I used standardised data (a frequency per 1,000 words).

The following section compares the distinctive lexico-grammatical features that are used to realise the main communicative purposes of the three obligatory moves in the Thai and New Zealand datasets. Apart from focusing on the prominent linguistic features of the three main moves in my dataset, I have also included an optional move, which is Move: Describing restaurant, in this analysis. This is because the distinctive linguistic features of this move manifest interesting cultural differences between the Thai and New Zealand hotels. The comparison between the two cultures is based on the frequency of wordlist and their collocations generated by AntConc 3.2.4. This register analysis particularly focuses on three main word classes: verbs, adjectives, and pronouns. Even though there is no fixed order of occurrence patterns of how the hotel use moves and steps to communicate their intentions in the datasets of Thailand and New Zealand, the following discussion of the four moves follows the most typical order pattern of moves that occurs in my dataset. Table 21 shows the four moves that I examine their lexico-grammatical features and collocations.

Table 21: Obligatory Moves and one optional move in the Thai and New Zealand dataset

\begin{tabular}{|l|c|c|}
\hline \multicolumn{1}{|c|}{ Moves } & $\begin{array}{c}\text { Thai hotels } \\
\text { (Total 30) }\end{array}$ & $\begin{array}{c}\text { New Zealand } \\
\text { hotels } \\
\text { (Total 30) }\end{array}$ \\
\hline Move: Introducing the hotel/facilities/services (I) & $\mathbf{3 0}$ & $\mathbf{2 7}$ \\
\hline Move: Describing the rooms (DR) & $\mathbf{3 0}$ & $\mathbf{3 0}$ \\
\hline Move: Establishing credentials (E) & $\mathbf{2 6}$ & $\mathbf{2 5}$ \\
\hline Move: Introducing restaurants/bars, and lounges (R) & 22 & 15 \\
\hline
\end{tabular}

As previously discussed in section 5.1, the obligatory moves are moves which occur more than 23 times (75\%) in the datasets. As such, Move: Introducing restaurants/bars, and lounges ( $R$ ) 
is an optional move because it occurs only 22 times (Thai hotels) and 15 times (New Zealand hotels) out of 30 .

\subsubsection{Move: Introducing the hotel/facilities/services (I)}

The discussion begins with the analysis of distinctive linguistic features in Move:

Introducing the hotel/facilities/services (I). It contains 2554 words from the overall datasets, 1130 words in the Thai dataset and 1424 in the New Zealand dataset respectively. This move focuses on introducing the hotel/facilities/services to the readers. This move can be realised by three steps:

Step ns: Stating the name/ or slogan of the hotels

Step gr: Greeting/welcoming the readers

Step ph: Providing a heading for different aspects of the hotels

\section{Verbs in Move: Introducing the hotel/facilities/services (I)}

The main focus of the analysis is to observe the linguistic behaviour of main verbs. They are classified as content words, which are frequently encountered in the Thai and in New Zealand websites. In terms of grammatical categories, content words refer to words that carry lexical meaning such as adjective, noun, adverb, and main verb. In contrast, function words do not convey meaning but link content words together; examples are auxiliary verb, modal verb, pronoun, preposition, conjunction, and determiners (Corver \& Riemsdijk, 2001; Segalowitz \& Lane, 2000; Stubbs, 2001). Since my datasets are small, main verbs usually have low frequency of occurrence and are situated lower in the frequency list. The most frequent words in the wordlist are generally function words, which is similar to Stubbs' (2001) study, where he investigated the meaning of words. The most frequent function verbs in my dataset are verb to be and verb to have. However, in Move: (I). I will only focus on the most frequent content verbs and their collocations. Table 22 shows that both datasets share two similar verbs: Welcome and Invite. There is a slight difference in terms of the collocational patterns in the way the verb Invite is used. It is interesting to note the verb stay is only encountered in the New Zealand dataset. All these verbs will be discussed in detail below. 
Table 22: The frequency and collocations of verbs in Move: (I) in the Thai and New Zealand datasets

\begin{tabular}{|l|l|c|l|}
\hline \multicolumn{4}{|c|}{ Thai hotels } \\
\hline Verbs & Collocates & $\begin{array}{c}\text { Per 1,000 } \\
\text { (frequency) }\end{array}$ & $\begin{array}{l}\text { T/BNC } \\
\text { (LL value) } *\end{array}$ \\
\hline Welcome & $\begin{array}{l}\text { + name of the } \\
\text { hotel }\end{array}$ & 16.81 & $\begin{array}{l}174.56 \\
\mathrm{p}<0.0001\end{array}$ \\
\hline Invite & $\begin{array}{l}\text { we + invite + } \\
\text { you to + the } \\
\text { hotel }\end{array}$ & 3.50 & $\begin{array}{l}37.53 \\
\mathrm{p}<0.0001\end{array}$ \\
\hline & & & \\
\hline
\end{tabular}

\begin{tabular}{|l|l|c|l|}
\hline \multicolumn{4}{|c|}{ New Zealand hotels } \\
\hline Verbs & \multicolumn{1}{|c|}{ Collocates } & $\begin{array}{l}\text { Per 1,000 } \\
\text { (frequency) }\end{array}$ & $\begin{array}{l}\text { NZ/BNC } \\
\text { (LL value) }\end{array}$ \\
\hline Welcome & - + name of the hotel & 17.50 & $\begin{array}{l}231.81 \\
\mathrm{p}<0.0001\end{array}$ \\
\hline Stay & $\begin{array}{l}24.28 \\
\text { - + with us at + name } \\
\text { of the hotel }\end{array}$ & 3.50 & $\begin{array}{l}24.28 \\
\mathrm{p}<0.0001\end{array}$ \\
\hline Invite & $\begin{array}{l}\text { Hosts invite + you+ } \\
\text { to V.1 (enjoy/join/ } \\
\text { stay) }\end{array}$ & 2.10 & $\begin{array}{l}25.05 \\
\mathrm{p}<0.0001\end{array}$ \\
\hline
\end{tabular}

*The $p$ value of the comparison of the frequency of verbs (Move: I) in the Thai and New Zealand datasets with the frequency of the same verbs in the BNC is $p<0.0001$. It indicates that the difference is highly significant.

*T/BNC (LL value) = Log-likelihood value comparing the Thai dataset with the BNC corpus

*NZ/BNC (LL value) = Log-likelihood value comparing the New Zealand dataset with the BNC corpus

Welcome is the most frequent verb that is used to achieve one of the communicative purposes in Move: (I) in both datasets and the verb Welcome is very distinctive in the boutique hotel websites compared to the BNC corpus. Welcome seems to be one of the most important verbs that is used in order to fulfill the main objective of Move I. Interestingly, Welcome exhibits similar collocations across the two datasets: welcome to+ name of the hotel as can be seen in the following example: "Welcome to the Bolton Hotel, the only luxury boutique hotel in Wellington" (NZ6) and "Welcome to Devasom Hua Hin Resort, a luxury boutique beach hotel where romantic Hua Hin charms and seaside serenity unite" (T1). These two examples from both datasets show that web designers usually provide a short description containing evaluative language (luxury, romantic) to inform the web audience about the distinctive characteristics of the hotels after the hotel name. This short description not only informs the readers about what the hotels are like but it also acts as a marketing technique to persuade them to book the hotels.

Next, the second most frequent verb in both datasets is Invite. Overall, it is used more frequently in the hotel promotional texts compared to the BNC. The collocational profiles of Invite show a slight difference of how Invite is used between the two datasets. In the Thai hotels we+ Invite+ you to v. infinitive is used, while in the New Zealand hosts +Invite +you to v. infinitive occurs frequently. For example, "We invite you to discover the magic of Zazen" (T12). "Your hosts Frances and Brian invite you to join them in their warm Kiwi hospitality" (NZ24). By looking at the function of these two examples, they seem to perform a similar function; namely to invite the readers to come experience the hotels' facilities and services which corresponds to the main purpose of step gr. However, in terms of semantic aspect, we+ Invite+ you to v. infinitive that is used in the Thai 
dataset is likely to be more formal than hosts +Invite +you to v. infinitive, which is encountered in the New Zealand dataset. The pronoun We refers to hotels or hotel staff members and connotes a less intimate relationship than Hosts. This is because in New Zealand, it is a cultural norm that informality is expressed by addressing everyone by their first name. Consequently, the collocational pattern that is used in the New Zealand boutique hotels seems more casual and shows a more intimate relationship between the hotels and the readers. This may be explained by the fact that the New Zealand hotels are established on a smaller scale (number of rooms) than the hotels in Thailand. It is very common for Thai boutique hotel owners to employ staff to run the hotels for them. However, in the New Zealand context, the owners of small boutique hotels usually operate their business by themselves, which builds a more intimate relationship with potential guests. The different collocation of the verb Invite connotes deviations between the approach the hotels in these two countries establish a relationship with their guests.

The final verb for analysis is Stay, which is only found in the New Zealand hotel websites. It is a dominant feature in Move (I) of the New Zealand websites because it occurs frequently $(3.5 / 1,000$ words) and it is very predominant when compared to the BNC. It appears to be a notable persuasive resource that web designers use to fulfill one of the communicative purposes of Move I which is to encourage the readers to make a reservation. Its collocational structure is Stay + with us at + name of the hotel. In addition, Us in this structure refers to the hosts who are inviting the readers. An example is "If you are visiting New Zealand and exploring the Westland region, stay with us at Teichelmann's Hokitika Bed \& Breakfast..." (NZ24). This structure is used by the New Zealand hotels as a casual invitation to persuade the guests to come and relax at their hotels.

\section{Adjectives in Move: Introducing the hotel/facilities/services (I)}

Table 23 demonstrates the most frequent adjectives that appear in the Thai boutique hotel websites: namely Boutique, Special, Thai, Tropical, and Romantic. The first two adjectives, Boutique and Special, also occur frequently in the New Zealand dataset and the last three, Thai, Tropical, and Romantic, only occur in the Thai dataset. 
Table 23: The frequency of adjectives and collocations in Move: (I) in the Thai and New Zealand datasets

\begin{tabular}{|l|l|c|l|}
\hline \multicolumn{3}{|c|}{ Thai hotels } \\
\hline Adjectives & Collocates & $\begin{array}{l}\text { Per 1,000 } \\
\text { (frequency) }\end{array}$ & $\begin{array}{l}\text { T/BNC } \\
\text { (LL value) * }\end{array}$ \\
\hline Boutique & $\begin{array}{l}\text { + name of the } \\
\text { hotels }\end{array}$ & $\begin{array}{l}418 \\
\mathrm{p}<0.0001\end{array}$ \\
\hline Special & $\begin{array}{l}\text { + offer/ } \\
\text { occasion }\end{array}$ & $\begin{array}{l}110.86 \\
\mathrm{p}<0.0001\end{array}$ \\
\hline Thai & $\begin{array}{l}\text { - life/style (slogan } \\
\text { of the hotels) }\end{array}$ & 4.40 & $\begin{array}{l}58.37 \\
\mathrm{p}<0.0001\end{array}$ \\
\hline Tropical & $\begin{array}{l}\text { - +garden/island } \\
\text { (slogan of the hotels) }\end{array}$ & 2.60 & $\begin{array}{l}24.30 \\
\mathrm{p}<0.0001\end{array}$ \\
\hline Romantic & $\begin{array}{l}\text { +atmosphere/ } \\
\text { place (slogan of the } \\
\text { hotels) }\end{array}$ & 2.60 & $\begin{array}{l}23.51 \\
\mathrm{p}<0.0001\end{array}$ \\
\hline
\end{tabular}

\begin{tabular}{|l|l|l|l|}
\hline \multicolumn{5}{|c|}{ New Zealand hotels } \\
\hline Adjectives & \multicolumn{1}{|c|}{ Collocates } & $\begin{array}{l}\text { Per 1,000 } \\
\text { (frequency) }\end{array}$ & $\begin{array}{l}\text { NZ/BNC } \\
\text { (LL value) }\end{array}$ \\
\hline Boutique & $\begin{array}{l}\text { +name of the } \\
\text { hotels }\end{array}$ & 19.60 & $\begin{array}{l}482.63 \\
\mathrm{p}<0.0001\end{array}$ \\
\hline Dining & $\begin{array}{l}\text { +location/ } \\
\text { room/menus }\end{array}$ & 5.60 & $\begin{array}{l}77.18 \\
\mathrm{p}<0.0001\end{array}$ \\
\hline Special & $\begin{array}{l}\text { + offer/ } \\
\text { occasion }\end{array}$ & 4.90 & $\begin{array}{l}30.40 \\
\mathrm{p}<0.0001\end{array}$ \\
\hline Warm & + welcome & 4.20 & $\begin{array}{l}37.94 \\
\mathrm{p}<0.0001\end{array}$ \\
\hline & & & \\
\hline
\end{tabular}

*The $p$ value of the comparison of the frequency of adjectives (Move: I) in the Thai and New Zealand datasets with the frequency of the same adjectives in the BNC is $p<0.0001$. It indicates that the difference is highly significant.

Boutique is the most frequent adjective in both datasets. It commonly appears as a part of the hotel name. Additionally, the function of Boutique in the collocational behavior corresponds to one of the steps of Move: (I), which is step ns: Stating the name/ or slogan of the hotels. Boutique is sometimes written with capital letter B, for example, "Club Bamboo Boutique resort \& spa in Phuket, Thailand" (T10), "Central Ridge Boutique hotel” (NZ14), while other times, it is written with a small B "P2 boutique hotel" (T19).

The second most frequent adjective in the Thai dataset is Special. Special is also a common adjective in the New Zealand dataset, however, it occurs more frequently in the Thai dataset (Thai 15/1,000 words and NZ 5.6/1,000 words) In both datasets, Special presents similar collocational patterns which are Special + offer/occasion as can be seen in the following phrase: "Rate and Special offer" (T15) and "Special occasions."(NZ2) Also, Special in these contexts functions as a heading for different sections in the boutique hotel websites, which is connected to step ph: Providing a heading for different aspects of the hotels. Interestingly, the hotels use special to attract readers' attention or to get an immediate response to the offers that the hotels aim to sell.

The last three most frequent adjectives in the Thai dataset are Thai, Tropical, and Romantic, which are mostly used in the hotel slogan to promote the theme and design of the hotels and which is related to step ns: Stating the name/ or slogan of the hotels. By using the following adjectives, hotels emphasise different themes to attract different target groups of customers. The first adjective is Thai whose collocational profile is Thai+ life/style, for example "Contemporary Thai Style, Relaxing and Romantic atmosphere" (T12) and "Welcome to the most beautiful Boutique Traditional Thai Life" (T14). This pattern is commonly used in hotel slogans to show that the hotels 
are decorated in a Thai style, which the hotels use as a selling point to attract prospective guests who would like to experience authentic Thai lifestyle hotels. Secondly, for the adjective Tropical, the collocation is tropical + garden/island as can be seen in the following example: "haven of natural harmony and home of peaceful style on Thailand most delightful tropical island" (T12). This structure appears to target guests who enjoy outdoor and adventurous activities in a tropical zone. The last adjective is, romantic, and the collocational pattern is romantic+atmosphere/place, which can be seen in this phrase "Relaxing and Romantic atmosphere" (T1). It seems that Romantic specifically attracts couples who would like to indulge themselves in a romantic retreat. Web designers use the word Romantic to create an ideal fascinating holiday experience in the guests' mind in order to persuade them come to experience their holidays at the hotels.

In addition to Boutique and Special that co-occur in the New Zealand dataset in Move (I), dining is the second most frequent adjective (5.6/1,000 words) in the New Zealand dataset. Also, dining is a very distinctive adjective in the boutique hotels promotional texts. The pattern of dining is dining + location/room/menus as can be seen in the phrase "ARTISAN restaurant + bar the perfect private dining location" (NZ6). This pattern is mainly used to present a title of sections in the hotel websites which is related to step ph: Providing a heading for different aspects of the hotels.

Another frequent adjective is Warm, which is a common adjective in the hotel advertising text connoting the feeling of cosiness and relaxation that the guests will experience from the hotel service and atmosphere. Its collocation is warm + welcome, which is shown in this example, "Louise Kelly with her family, friends and delightful staff offer a warm welcome to their well-established Boutique Bed \& Breakfast Accommodation..." (NZ 19). From the example, the hotel seems to use this pattern to offer their hearty and hospitable reception. Also, the hotels aim to construct a friendly relationship between hotels and guests in order to convince them to stay at this hotel.

\section{Pronouns in Move: Introducing the hotel/facilities/services (I)}

Myers (1994), Cook (1992), Pitchinee, (1995), Fuertes-Olivera, Velasco-Sacristán, ArribasBaño and Samaniego-Fernández, (2001) claim that personal pronouns such as You, Your, We and Our are a common persuasive strategy in making a sense of solidarity with prospective customers. These findings are also supported by my analysis below.

Table 24 shows the most frequent pronouns that occur in the Thai and New Zealand datasets; namely Our, Your, We, and You. The occurrence of pronouns in the boutique hotel websites in comparison to the $B N C$ indicate that the use of all pronouns in the Thai dataset is a distinctive linguistic feature. The concordance lines reveal that most pronouns share a similar pattern of collocations across two except for Your. 
Table 24: The Frequency of pronouns and collocations in Move: (I) in the Thai and New Zealand datasets

\begin{tabular}{|l|l|l|l|}
\hline \multicolumn{5}{|c|}{ Thai hotels } \\
\hline Pronouns & Collocates & $\begin{array}{l}\text { Per 1,000 } \\
\text { (frequency) }\end{array}$ & $\begin{array}{l}\text { T/BNC } \\
\text { (LL value) }\end{array}$ \\
\hline our & -+ hotel & 7.00 & $\begin{array}{l}18.67 \\
\mathrm{p}<0.0001\end{array}$ \\
\hline Your & $\begin{array}{l}\text { + body and } \\
\text { mind/ inner } \\
\text { self/lifestyle/pre } \\
\text { ference/dreams }\end{array}$ & 6.00 & $\begin{array}{l}14.73 \\
\mathrm{p}<0.0001\end{array}$ \\
\hline You & $\begin{array}{l}\text { we/hotels+ } \\
\text { welcoming } \\
\text { statement }+ \text { you }\end{array}$ & 14.00 & $\begin{array}{l}7.24 \\
\mathrm{p}<0.01\end{array}$ \\
\hline We & $\begin{array}{l}+ \text { welcoming } \\
\text { statement }\end{array}$ & 6.00 & $\begin{array}{l}14.73 \\
\mathrm{p}<0.0001\end{array}$ \\
& & & \\
\hline
\end{tabular}

\begin{tabular}{|l|l|l|l|}
\hline \multicolumn{5}{|c|}{ New Zealand hotels } \\
\hline Pronouns & \multicolumn{1}{|c|}{ Collocates } & $\begin{array}{l}\text { Per 1,000 } \\
\text { (frequency) }\end{array}$ & $\begin{array}{l}\text { NZ/BNC } \\
\text { (LL value) }\end{array}$ \\
\hline Our & $\begin{array}{l}\text { + hotels/ } \\
\text { facilities and } \\
\text { services }\end{array}$ & 8.40 & $\begin{array}{l}31.71 \\
p<0.0001\end{array}$ \\
\hline Your & $\begin{array}{l}\text { A)_+ holiday } \\
\text { experiences } \\
\text { (visit/stay) } \\
\text { B) your+ hosts }\end{array}$ & 4.90 & $\begin{array}{l}8.13 \\
p<0.01\end{array}$ \\
\hline You & $\begin{array}{l}\text { Host name/we + } \\
\text { welcoming } \\
\text { statement + you }\end{array}$ & & $\begin{array}{l}0.09 \\
p>0.05\end{array}$ \\
\hline We & $\begin{array}{l}\text { we+ welcoming } \\
\text { statement }\end{array}$ & 3.50 & $\begin{array}{l}0.00 \\
p>0.05\end{array}$ \\
& & & \\
\hline
\end{tabular}

*The $p$ value of the comparison of the frequency of the pronoun (Move: I) in the Thai and New Zealand datasets with the frequency of the same pronouns in the BNC is between $p>0.05$ and $p<0.0001$

The most distinctive pronoun in the hotel promotional texts is Our, which reflects a sense of belonging. Our in this context is an exclusive pronoun (excluding the readers) and mainly refers to hotels and hotel hosts in the New Zealand dataset and hotels and hotel staff in the Thai dataset. The concordance lines exhibit minor differences between the use of our across the two datasets. In the Thai boutique hotel advertising webpages, the collocation of Our is our + hotel, whereas ourt hotels/facilities and services is used in the New Zealand hotel webpages. Even though there are slight differences in the way Our is used between the two datasets, its function is the same, which is to welcome the web audience to stay in the hotels and use their facilities. When Our is used exclusively the function is related to the communicative purposes of one the steps within Move: (I) which is step gr: Greeting/welcoming the readers. For example, "Panther Creek welcome to our Western Boutique Resort..."(T20) “...we invite you to enjoy our hospitality and share with us our new Hadleigh..."(NZ7). The sense of belonging and self-identification in the boutique hotel websites is enhanced by the dominance of the pronoun our exclusive and this seems to be an important characteristic that the web designers use to distinguish themselves from other hotels.

The second most frequent pronoun is Your. It refers to the web audience whom the hotels aim to target. The collocations of Your demonstrates major differences across the Thai and New Zealand datasets. In the Thai hotel texts, the collocational pattern is your + body and mind/ inner self/lifestyle/preference/dreams. This pattern is mainly used to promote slogans of the hotels and to invite the potential guests to visit the hotels, which correspond to two of the steps that are used as 
rhetorical devices to achieve the communicative purposes of Move: (I): namely step ns: Stating the name/ or slogan of the hotels (body and mind/inner self) and step gr: Greeting/welcoming the readers (lifestyle/preference/dreams). Examples are "Zazen-Escape Into Your Inner Self" (T12) (step $n s)$ and "We invite you to the holidays of your dreams at Zazen Boutique Resort \&Spa, Ko Samui" (T12) (step gr).These two examples of your highlight that the hotel has a clear idea about what they view the customers' wants to be, and they respond to their market needs. The hotels use your to emphasise the keywords to respond to their guests' desires through the use of slogans and welcoming statement in order to deliver the advertising impact as quickly as possible.

On the other hand, the collocational structures of your in the New Zealand dataset focus on the holiday experience and the hotel hosts rather than on the guest personal desires, as can be seen in the following patterns: A) Your+ holiday experiences (visit/stay/holiday) and B) your+ hosts. These two patterns are illustrated in these phrases; namely 1) "Waimana Point Lodge Luxury accommodation for your New Zealand holiday" (NZ25), 2) "Some special features to make your stay with us convenient and enjoyable..."(NZ2), and 3) "Your hosts Frances and Brian invite you to join them in their warm and inviting character home..." (NZ24). These three examples enhance the three main communicative purposes of Move: (I). Also, the second collocational pattern (B), where the names of the hosts are usually presented, enhances the friendly relationship and personal invitation that web designers intend to construct and deliver to the readers. Interestingly, the overall occurrence of Your accomplish all the communicative purposes conveyed in Move: (I).

The third most frequent pronoun in the Thai dataset is $W e$, which is a distinctive pronoun in the Thai dataset, while We (the fourth most frequent pronoun) in the New Zealand dataset is not prevalent as opposed to the BNC. The collocation of the pronoun We between the two datasets reveals the similar pattern We+ welcoming statement. The main communicative function of this pattern is to welcome and invite the potential guests to stay at the hotels. Examples are "We cordially invite you to our unique Hua Hin hotel on a private beach" (T1). and "We offer you superior Taupo motel accommodation and a friendly welcome here at Beechtree Suites" (NZ22). The concordance lines reveal different kinds of referents of we presented in the Thai hotel websites, which are "small and secluded boutique hotels", "boutique resort on the beach", "luxury boutique hotels", "western boutique resort", and "Thai traditional boutique hotel". In contrast, in the New Zealand dataset, the pronoun we generally refers to "motel accommodation", "heritage boutique collection property", and "bed and breakfast." These referents can be observed by the fact that We usually appears close to the name of the hotels or the description of the hotels. 
The least frequent pronoun in the Thai dataset is You. However, the occurrence of pronoun You within the Thai dataset itself is dominant (14/1,000 words); it is the most frequent pronoun based on the number of tokens that occur in the Thai dataset, but it is the least when compared with the BNC (both written and spoken register). Similarly, this scenario appears to be common in the New Zealand dataset. Based on the number of occurrence of You (7/1,000 words) in the New Zealand dataset You is the second most frequent pronoun though less frequent compared to the BNC. This may explain that You is a distinctive addressing term used in the BNC compared to promotional texts. Although the pronoun You demonstrates considerable differences in terms of its frequency of occurrence across the Thai and New Zealand daraset, collocational behaviours are relatively similar which are we/hotels + welcoming statement + You (T) and hosts' name/we + welcoming statement+ You (NZ). For example, "Panther Creek, the Western Boutique Resort is welcome you to our 48 acres cowboy town..."(T20), and "Dave and Lea Bentley, both $4^{\text {th }}$ generation Kiwis, welcome you to Misty Peaks"(NZ16). These examples reflect the communicative purpose of step gr: Greeting/welcoming the readers within Move: (I). Additionally, they show the main difference in the way you is addressed in welcoming statements across the two datasets. In the Thai dataset, the name of the hotels is frequently used as a subject of the sentences, while in the New Zostsnid databednthedeptedf this may be explained by the differences in the management structure of the boutique hotels between these two countries that I mentioned earlier in previous paragraph. Many boutique hotels in the New Zealand dataset are small and run directly by the owners, which is likely to enhance intimate relationship between the potential guests and the hotel owners. On the other hand, most Thai hotels are larger operations and have staff to represent their hotels and run day by day work for the hotel owners. As a result, the way the hotel owners construct the relationship between the hotels' owners and the guests in these two datasets appears to be different. The overall use of verbs, adjectives, and pronouns on the Thai hotels and New Zealand hotel website in Move: (I) reflects the distinctive characteristics of each country. The variations in the uses of prominent linguistic features and collocational patterns between these two countries are influenced by the differences in the business scales of the hotels.

\subsubsection{Move: Establishing credentials (E)}

The next move that I will focus on is Move: Establishing credentials (E). As previously discussed in section 5.1, I borrowed the name of this move from Bhatia's (1993) sales promotion letter analysis because they share common communicative purposes. The main purpose of this move in the context of boutique hotel online advertisement is to construct positive images of the hotels and to prove to readers that the hotels are well established with outstanding, unique services 
and facilities. Move: (E) convinces the guests to decide to book the hotels. This move thus provides information on different aspects of the hotel such as guest comments, location, scenery, history, and awards received. Move: (E) contains 12,324 words from the overall dataset. These words are further subdivided into 5,326 words in the Thai dataset and 6,988 in the New Zealand dataset. This move can be illustrated by eight steps:

Step dl: Describing the location

Step in: Informing the readers about the background or history of the hotels and the hosts

Step ic: Providing local knowledge and cultural background

Step cs: Claiming superiority

Step cl: Claiming luxury

Step ia: Introducing external awards received

Step ig: Introducing guests' feedback

Step sa: Providing satisfaction assurance

\section{Verbs in Move: Establishing credentials (E)}

Table 25 shows the frequent verbs and their main collocations that are found in Move: $(E)$ in the Thai and New Zealand datasets. Similar verbs (Has, Located, and Offer) are found in both countries.

Table 25: The Frequency of verbs and collocations in Move: (E) in the Thai and New Zealand datasets

\begin{tabular}{|c|c|c|c|}
\hline \multicolumn{4}{|c|}{ Thai hotels } \\
\hline Verbs & Collocates & $\begin{array}{c}\text { Per 1,000 } \\
\text { (frequency) }\end{array}$ & $\begin{array}{l}\text { T/BNC } \\
\text { (LL value) * }\end{array}$ \\
\hline Has & $\begin{array}{l}\text { (1) to introduce } \\
\text { facilities and } \\
\text { service } \\
\text { (3) to describe } \\
\text { background and } \\
\text { history of the } \\
\text { hotels) }\end{array}$ & 4.60 & $\begin{array}{l}7.85 \\
p<0.01\end{array}$ \\
\hline Located & - location & 3.90 & $\begin{array}{l}171.37 \\
p<0.0001\end{array}$ \\
\hline Offer & $\begin{array}{l}\text { + noun } \\
\text { (accommodation } \\
\text { and services) }\end{array}$ & 0.50 & $\begin{array}{l}3.42 \\
p>0.05\end{array}$ \\
\hline
\end{tabular}

\begin{tabular}{|c|c|c|c|}
\hline \multicolumn{4}{|c|}{ New Zealand hotels } \\
\hline Verbs & Collocates & $\begin{array}{l}\text { Per 1,000 } \\
\text { (frequency) }\end{array}$ & $\begin{array}{l}\text { NZ/BNC } \\
\text { (LL value) * }\end{array}$ \\
\hline Has & $\begin{array}{l}\text { (1) to introduce } \\
\text { facilities and } \\
\text { service) } \\
\text { (2) to describe } \\
\text { background and } \\
\text { history of the } \\
\text { hotels) }\end{array}$ & 3.40 & $\begin{array}{l}2.02 \\
p>0.05\end{array}$ \\
\hline Located & _+location & 2.80 & $\begin{array}{l}150.49 \\
p<0.0001\end{array}$ \\
\hline Offer & $\begin{array}{l}\text { + noun } \\
\text { (accommodation } \\
\text { and services) }\end{array}$ & 2.20 & $\begin{array}{l}56.50 \\
p<0.0001\end{array}$ \\
\hline
\end{tabular}

*The $p$ value of the comparison of the frequency of the verbs (Move: $\mathrm{E}$ ) in the Thai and New Zealand datasets with the frequency of the same verbs in the BNC is between $p>0.05$ and $p<0.0001$ 
The most frequent verb is Has. In this respect, Has is used in two main different aspects in both datasets: 1) to introduce facilities and service, and 2) to describe background and history of the hotels. Web designers use Has in the present tense form to promote facilities, services, and location that the hotels have to offer. For example, "our property has its own private vineyard"(NZ1). In addition, two examples of Has reflect the main purpose of step cs claiming superiority within Move: (E) as follows. "Our resort has one of the best-equipped fitness facilities on the island" (T15) and "The Bolton Hotel has an ideal Wellington city centre location..."(NZ6). Apart from generally describing the hotel offers, the hotel websites also intend to claim that their hotels are better than the competitors through the use of Has with adjectives such as best and ideal. This technique creates the persuasive force on the readers and influences their decision making on booking the hotels.

The second function of Has is to narrate the history and background of the hosts or the hotels. This function is connected to the main communicative purpose of step ih Informing the readers about the background or history of the hotels and the hosts. The web designers aim to highlight the value of historical background of the hosts or the hotels and make the readers feel close and desire to witness and take part in this long established historical setting. For example, "This historical building has been restored maintaining the original Chiang Mai architectural style while giving it a modern Thai accent" (T25). A value of architectural design is frequently reflected through the narration of historical background of the hotels, which is considered as one of the most important aspect hoteliers should bear in mind. In addition, narrating the historical background of the hotels and the hosts helps to construct a relationship between the hosts and the audience, like in this phrase, "The Prices Gate Hotel has been in the Marvelly family for nearly thirty years. Their family welcomes yours and hopes your stay will be truly magical one" (NZ20).

The second most frequent verb found in the Thai and New Zealand datasets is Located. Even though these two datasets share the same verbs and collocational profile L+location), they reveal different degree of frequency of occurrence. In the Thai dataset Located occurs 3.9 times in 1,000 words compared to $2.8 / 1,000$ words in the New Zealand dataset. Located is used to enhance the location of the hotels that they are situated in a prime area with ease of transportation. For example, "Conveniently located near the Wellington $C B D$, minutes walk from The Terrace, Lambton Quay and Parliament." (NZ18), and "Conveniently located on Petchburi Road Pratunam Area, this Bangkok hotel is a walk away from BT, shopping Centers, MBK and Tourist Attractions" (T14). These examples are also linked to the communicative function of step: describing location within Move (E), wherein web designers use located as a linguistic technique to persuade potential customers to come and stay in the hotels. 
Lastly, the third most frequent verb is Offer. It mainly occurs in the New Zealand hotel websites. Offer occurs only $0.5 / 1,000$ words in the Thai dataset. It usually collocates with nouns (accommodation and services) in order to provide information about accommodation and services. An example is, "Elizas Manor Boutique Hotel offers quality, comfortable central Christchurch bed and breakfast accommodation (NZ9)." Interestingly, the concordances also provide evidence that offer is used as a linguistic device to claim that the hotels provide the best services and unique quality; this is related to the main communicative purpose of Move E. For example, "Central Ridge Boutique Hotel offers its famous unique luxury experience with a quality bed and breakfast and day spa facility"(NZ14).

\section{Adjectives in Move: Establishing credentials (E)}

Table 26 shows a list of frequent adjectives and their collocational profiles that are used in Move: (E) in the Thai and New Zealand hotel datasets. Both datasets use relatively the same degree of frequency of adjectives as an act of persuasion even though the overall size of New Zealand dataset is larger. Interestingly, the adjectives found in the New Zealand dataset are more related to the main communicative purpose of step cs claiming superiority than the Thai dataset because the New Zealand hotels use Perfect, Best, and Unique to promote their hotels. Web designers commented on their belief that uniqueness and superiority are important in competing with other types of hotels. Even though the list of frequent adjectives varies across the two datasets, it also shares a similarity; that is, both countries use the adjectives Boutique and Unique. The most frequent adjective in both datasets is Boutique, which reveals a similar pattern of collocation. The other two adjectives that only occur in the Thai dataset are Thai and Tropical. The collocations of these adjectives are discussed in the next paragraph. 
Table 26: Frequencies of adjectives and their collocation in Move: (E) in the Thai and New Zealand datasets

\begin{tabular}{|c|c|c|c|}
\hline \multicolumn{4}{|c|}{ Thai hotels } \\
\hline Adjectives & Collocates & $\begin{array}{c}\text { Per } 1,000 \\
\text { (frequency) }\end{array}$ & $\begin{array}{l}\text { T/BNC } \\
\text { (LL value)* }\end{array}$ \\
\hline Boutique & $\begin{array}{l}\text { +noun } \\
\text { (identifying types } \\
\text { of hotels) }\end{array}$ & 5.20 & $\begin{array}{l}408.77 \\
p<0.0001\end{array}$ \\
\hline Thai & $\begin{array}{l}\text { +noun } \\
\text { (to describe a } \\
\text { design of } \\
\text { accommodation) }\end{array}$ & 4.80 & $\begin{array}{l}307.49 \\
p<0.0001\end{array}$ \\
\hline Tropical & $\begin{array}{l}\text { - noun (natural } \\
\text { environment and } \\
\text { weather) }\end{array}$ & 2.80 & $\begin{array}{l}122.14 \\
p<0.0001\end{array}$ \\
\hline Unique & $\begin{array}{l}\text { a)_noun } \\
\text { (describing design } \\
\text { and atmosphere } \\
\text { of the hotels) }\end{array}$ & 2.40 & $\begin{array}{l}79.67 \\
p<0.0001\end{array}$ \\
\hline
\end{tabular}

\begin{tabular}{|c|c|c|c|}
\hline \multicolumn{4}{|c|}{ New Zealand hotels } \\
\hline Adjectives & Collocates & $\begin{array}{l}\text { Per 1,000 } \\
\text { (frequency) }\end{array}$ & $\begin{array}{l}\text { NZ/BNC } \\
\text { (LL value)* }\end{array}$ \\
\hline Boutique & $\begin{array}{l}\text { +noun } \\
\text { (identifying types } \\
\text { of hotels) }\end{array}$ & 5.70 & $\begin{array}{l}587.32 \\
p<0.0001\end{array}$ \\
\hline Perfect & _+noun (location) & 4.10 & $\begin{array}{l}193.45 \\
p<0.0001\end{array}$ \\
\hline Best & $\begin{array}{l}\text { _tnoun (related } \\
\text { to service quality, } \\
\text { location of the } \\
\text { hotels and main } \\
\text { tourist attraction } \\
\text { nearby) }\end{array}$ & 2.70 & $\begin{array}{l}45.37 \\
p<0.0001\end{array}$ \\
\hline Unique & $\begin{array}{l}\text { a)_noun (touristic } \\
\text { experience) }\end{array}$ & 1.40 & $\begin{array}{l}50.86 \\
p<0.0001\end{array}$ \\
\hline
\end{tabular}

*The $p$ value of the comparison of the frequency of adjectives (Move: $\mathrm{E}$ ) in the Thai dataset and New Zealand dataset with the frequency of the same adjectives in the BNC is $p<0.0001$. It indicates that the difference is highly significant.

As noted, Boutique is the most frequent adjective, occurring 5.2 times per 1,000 words in the Thai websites and 5.7/1,000 in the New Zealand websites. Boutique is used to modify a variety of hotel accommodation terms to emphasise one of a kind accommodation, such as boutique bed and breakfast, boutique lodge, boutique hotel, boutique beach resort, and boutique cowboy town. Web designers aim to use the word boutique to amplify the unique characteristics of being a boutique hotel as a major selling point to compete with other types of hotels. As Olga (2009) claims, an increasing number of travellers expect to experience a new trend of hotel which offers fashionable lifestyle rather than simply comfort and convenience. Boutique hotels are actively responsive to this demand of the new form of travelling. In Olga's (2009) comment about the boutique hotel phenomenon, she notes that boutique hotels are considered as "social manipulation" (p. 2021). People want to stay at boutique hotels because they want to be recognised as fashionable and sophisticated (see more studies related to boutique hotels in section 2.3 chapter 2).

Another similar adjective is Unique that co-occurs in both Thai (2.4/1,000 words) and New Zealand (1.4/1,000 words) datasets. The collocation structure of this adjective between these two countries is different. In the Thai hotels, the pattern is unique+ noun (describing design and atmosphere of the hotels). For example, "the garden was developed into an innovative and unique French Tropical style" (T8). On the other hand, in the New Zealand context, the collocation is 
unique+ noun (touristic experience) as can be seen in the following example: "a unique underworld experience" (N13). The two different collocations and their examples show that the hotels in these two cultures have a contrastive emphasis in using the adjective Unique to promote their hotels. In the Thai dataset, the hotels seem to focus on design of the hotels and their atmosphere. The hotels may aim to persuade the readers that it is very easy to have a wonderful experience within the hotels because the guests can simply indulge themselves in extraordinary architecture. However, in the New Zealand dataset, the main selling point is not a design of the hotels; the major focus seems to be outdoor activities that the guests can explore. This adjective Unique seems to well portray a preferred lifestyle of tourists in both countries, which is similar to Hall and Kearsley's (2001) suggestion that one of the popular reasons for traveling in New Zealand is outdoor adventures ( $p$. $63)$.

The second most frequent adjective is Thai, which has a frequency of $4.8 / 1,000$ words. Only encountered in the Thai dataset, it describes the design and decoration of boutique hotels, for example Thai houses, Thai accent, Thai style, and Thai architectural design. These occurrences are in accordance with the description of the design of a few boutique hotels in my dataset, which are built and decorated in Thai traditional style. This may explain that hotel web designers believe that Thai traditional unique design is important to attract a niche of visitors seeking a special and differentiated accommodation.

The third most frequent adjective is Tropical which has a frequency of $2.8 / 1,000$ words. It only occurs in the Thai dataset. The linguistic behaviour of this adjective is used to describe the surrounding environment and atmosphere of the hotels. The main collocations of Tropical are garden, vegetation, tree, green forest, and weather. Additionally, Tropical enhances a feeling of seclusion and relaxation of the accommodation and makes the readers imagine themselves strolling around the green location and atmosphere of the hotels. It can be observed in this example "sit and surrounded by a tropical green forest where nature and life have met" (T20).

In regard to the New Zealand dataset, the second most frequent adjective is Perfect which accounts for $4.10 / 1,000$ words. The third most frequent adjective is Best whose frequency is $2.7 / 1,000$ words. These two adjectives are mostly frequently found in step cs: claiming superiority, which aims to create a positive image and highlights the uniqueness of facilities and services offered by the hotels. Since the main objective of Move E: Establishing Credentials is to distinguish their hotels from other traditional hotels which simply offer comfort and convenience, web designers aim to promote the outstanding features that other hotels do not have. Consequently, the hotel websites emphasise the use of the adjectives Perfect and Best to draw their target customers' 
attention. In this Move, Perfect is used to describe how good the location of the hotels are because the hotels know that visitors usually consider the location of their vacations or business as one of the major concerns, as noted in Olga's (2009) study. Olga (2009) states that a site of hotels is considered as high on "guests' priority lists" (p. 2022). The visitors choose their holiday destination not only based on convenient aspects but also on fashionable neighbourhood or surrounding area. Web designers anticipate these needs and then highlight that their hotels are situated in ideal locations. For example, "Accommodation at the Bolton Hotel in Wellington provides the perfect base for you to explore the city" (NZ6). Also, Perfect is frequently used to portray a wedding venue to persuade guests to come and celebrate their wedding ceremony at the hotels. Web designers focus on combining romantic atmosphere with ideal location for having a perfect and memorable wedding, as can be seen in this phrase, "A magical day deserves a magical location. The Princes Gate Hotel, with its classic sophistication, is the perfect place to celebrate love and romance"(NZ20). Similarly, the adjective Best is also used to claim that a quality of service and hotel location is the best, so the readers can experience the most wonderful holidays at the hotels. For example, "Misty Peaks located near Lake Matheson, offers you the very best in quality, comfort, and genuine Kiwi hospitality"(T16).

By analysing adjectives that occur in Move: $(E)$ in the Thai and New Zealand hotels, this analysis shows that the Thai web designers tend to use a more subtle strategy in this move to claim that they are better than the other hotels. Instead of claiming that they are the best hotel, the Thai hotels tend to enhance their strong qualities, such as Thai, Tropical, and Unique when describing the design and atmosphere. Interviews with the hotel owners showed they believe that the tourists would like to witness the Thai traditional design of the hotels and enjoy a Tropical climate when visiting Thailand. These two qualities are the major selling points of the hotels. They avoid using Best and Perfect, adjectives which are frequently found in the New Zealand hotels. This variation can be explained by the difference in cultural background between the two countries as suggested by Chaidaroon (2003) that Thai culture is less assertive; they prefer to be humble when communicating with people.

\section{Pronouns in Move: Establishing credentials (E)}

Table 27 shows the collocational patterns of pronouns in Move: $(E)$. The hotel websites in New Zealand generally use pronouns more (You 6.50, Our 6.70, and We 7.70 out of 1,000 words) than the Thai hotel websites (You 3.70, Our 3.50, and Your 3.20). Based on a comparison in terms of the occurrence of pronouns and their frequency, You and Our appear frequently in both datasets. The occurrence of the pronouns explain the different emphasis between the two 
datasets. It seems that in the Thai hotels the main focus is the readers (You as a subject) who will get the benefits of the offers being promoted by the hotels (Our). Therefore, the most frequent pronouns are You, Our, and Your. In contrast, in New Zealand hotel websites, We, the most frequent pronoun is used as the subject of sentences, which refers to hotels. The hotel owners aim to emphasise a provider who offers a higher quality of service. The hotels intend to show the readers that their facilities and services are more outstanding than that of other hotels, which is related to the main function of Move: (E). Accordingly, the focus is on We and Our.

Table 27: Frequencies of pronouns and their collocations in Move: (E) in the Thai and New

\section{Zealand datasets}

\begin{tabular}{|c|c|c|c|}
\hline \multicolumn{4}{|c|}{ Thai hotels } \\
\hline Pronouns & Collocates & $\begin{array}{r}\text { Per } 1,000 \\
\text { (frequency) }\end{array}$ & $\begin{array}{l}\text { T/BNC } \\
\text { (LL value)* }\end{array}$ \\
\hline You & $\begin{array}{l}\text {-can }+ \text { v.inf } \\
\text { (experiential verbs) }\end{array}$ & 3.70 & $\begin{array}{l}7.79 \\
p<0.01\end{array}$ \\
\hline Our & $\begin{array}{l}\text { noun (types of } \\
\text { hotels) }\end{array}$ & 3.50 & $\begin{array}{l}23.21 \\
p<0.0001\end{array}$ \\
\hline Your & $\begin{array}{l}\text { a)_noun (wedding } \\
\text { related experience) } \\
\text { b)_noun (touristic } \\
\text { related) }\end{array}$ & 3.20 & $\begin{array}{l}10.00 \\
p<0.01\end{array}$ \\
\hline We & $\begin{array}{l}\text { a)_verb (describing } \\
\text { experience while } \\
\text { visiting the hotels } \\
\text { b)_verb (informing a } \\
\text { background of the } \\
\text { hotels to establish } \\
\text { credentials } \\
\text { c)_verb (describing } \\
\text { location) }\end{array}$ & 2.40 & $\begin{array}{l}1.86 \\
p>0.05\end{array}$ \\
\hline
\end{tabular}

\begin{tabular}{|c|c|c|c|}
\hline \multicolumn{4}{|c|}{ New Zealand hotels } \\
\hline Pronouns & Collocates & $\begin{array}{l}\text { Per 1,000 } \\
\text { (frequency) }\end{array}$ & $\begin{array}{l}\text { NZ/BNC } \\
\text { (LL value)* }\end{array}$ \\
\hline We & $\begin{array}{l}\text { a)_verb (describing } \\
\text { location) } \\
\text { b) verb (informing a } \\
\text { background of the } \\
\text { host to establish } \\
\text { credentials } \\
\text { c)_verb (describing } \\
\text { experience while } \\
\text { visiting the hotels }\end{array}$ & 7.70 & $\begin{array}{l}27.04 \\
p<0.0001\end{array}$ \\
\hline Our & $\begin{array}{l}\text { noun (types of } \\
\text { hotels) }\end{array}$ & 6.70 & $\begin{array}{l}105.58 \\
p<0.0001\end{array}$ \\
\hline You & $\begin{array}{l}\text { (experiential verb) } \\
\text { (ex.inf }\end{array}$ & 6.50 & $\begin{array}{l}0.02 \\
p>0.05\end{array}$ \\
\hline Your & $\begin{array}{l}\text { a)_noun (touristic } \\
\text { related) } \\
\text { b)_noun (hotel } \\
\text { facilities and } \\
\text { services related) in } \\
\text { guest reviews } \\
\text { section }\end{array}$ & 2.70 & $\begin{array}{l}7.81 \\
p<0.01\end{array}$ \\
\hline
\end{tabular}

*The $p$ value of the comparison of the frequency of the pronouns (Move: $\mathrm{E}$ ) in the Thai and New Zealand datasets with the frequency of the same pronouns in the BNC is between $p>0.05$ and $p<0.0001$

One distinctive difference between the two datasets in Move: $(E)$ is the use of pronouns.

Since the size of the Thai datset is smaller as well as the use of pronouns being less frequent compared to the New Zealand dataset, the use of pronouns does not highly reflect the characteristics of the main purpose of Move: $(E)$ which is to show different aspects that make hotels 
more outstanding than other boutique hotels. Rather, pronouns are likely to be used to describe the special facilities and activities that the potential visitors ( $\mathrm{YOU}$ ) can enjoy during their visit. For example, "You will discover true serenity in the beautiful city of Chiang Mai" (T7). In contrast, pronouns are used more frequently in the New Zealand hotels. The use of pronouns clearly reflects the communicative purposes of Move E: Establishing credentials by stating two of the steps within this move step: describing the location, and step in: Informing the readers about the background or history of the hotels and the hosts through pronoun We which refers to the hotels and the hosts. For example, "We are also closely situated to Wellington's beautiful Botanic Gardens and local Thorndon treasure Katherine, Mansfield's birthplace"(NZ18). Even though the Thai dataset also contain similar instances (the use of the pronoun We), there are very few, as the pronoun We has a lower frequency than You, Our, Your in the Thai dataset. It is interesting to note that the Thai hotels adopt other persuasive strategies to achieve the purpose of this move instead of pronouns as previously discussed in the above section.

Table 27 demonstrates that the most frequent pronoun in the Thai dataset is You, while it is the third most frequent in the New Zealand hotels. The hotel websites in both countries use You to address their target audience and inform them about the offers that they will experience when they visit the hotels. In regard to the referents of You in the Thai dataset, You frequently refers to "individuals", "couples", and "family". For example, "You can celebrate your wedding or honeymoon in style"(T4). On the other hand, in the New Zealand dataset, the use of You includes "individuals", "couples", "families", and "corporates", as can be seen in this example, "you have the perfect venue for all types of occasions" (NZ5). The collocation of You in both datasets reveals similarities; that is, You commonly collocates with can $+v$.inf (experiential verbs) to indicate that the readers will encounter surrounding environments, facilities, activities that the hotels prepare or the regions have offered for them. An example is, "Here you can enjoy stunning panorama views of Tasman bay..."(NZ3), which corresponds to one of the main communicative purposes of Move: (E), which is describing location.

The second most frequent pronoun is Our, which is also found in both datasets but it occurs with a higher frequency in the New Zealand hotel websites: (6.70 in the New Zealand hotel texts compared to 3.5 in the Thai hotel texts). Our reveals a similar collocation pattern, which is our + noun (types of hotels). Web designers use Our to signify the ownership of the hotels which mainly refers to their hotels and their properties such as (property, lodge, pavilion style home, motel, Bed and Breakfast, accommodation, and hotel) in New Zealand and (resort, floatel, and hotel) in the Thai datasets. These examples show the differences between types of accommodation presented between the two datasets reflecting the nature of accommodation in each country. In New Zealand, 
the most frequent term referring to boutique hotels is "property", while in Thailand the most frequent term is "resort". The term "property" reflects the characteristics of New Zealand hotels, which are owned by local people who usually convert their properties into boutique hotels and open them to the guests who would like to experience true kiwi hospitality. On the other hand, boutique hotels in Thailand use "resort" to highlight their prime locations, which are ideally situated in mountains or near the beaches or rivers to attract visitors.

The third most frequent pronouns that are found in the texts of Move: $(E)$ in the Thai hotel websites is Your, while it is less frequent in the New Zealand hotel webpages (2.70/1,000 words). The collocate behaviour of Your in both datasets reveals only one similar pattern among two typical patterns that occur in both datasets; that is Your commonly collocates with noun (tourismrelated) to indicate, "We are a perfect answer for your vacation" (T4). "Their family welcomes yours and hopes your stay will be a truly magical one" (NZ20). The hotels use this pattern to convince the guests that their hotels are the best destination that can make the guests' stay the most wonderful. In the Thai dataset, the most distinctive collocation of Your is your + noun (wedding-related experience). It is used to highlight the best location and beautiful scenery of the wedding provided by the hotels; an example is: "the warm tropical weather makes everything seem more vibrant, the beach acting as your backdrop" (T4). This example is used to emphasise the main advantage of the hotel that it is located in a great location with beautiful scenery. The hotel uses this example to target couples who have a dream to have a wedding reception outdoors. On the other hand, the most crucial pattern of Your in the New Zealand hotels is your + noun (hotel facilities and services related). It solely appears in a guests' review section, which the hotels use to boost their credibility. This pattern is equivalent to step: introducing guests' feedback. An example of this is: "We will be back for more indulgence and your wonderful hospitality" (NZ2). Interviews with the hotel owners in Thailand and New Zealand also support this occurrence. The owners stated that it is very important for the guests to do research before making a reservation; correspondingly, the hotels usually post guests' comments on their websites to reassure the readers. Some hotels have their in-house guests' reviews section on their websites in order for guests to complain or compliment. The viewers can click on the link (guests' reviews) to see all feedback. On the other hand, many hotels rely on guests' reviews posted on Trip Advisor website; therefore, the hotels put a link to guests review posted on Trip Advisor on their hotel website. This marketing strategy can work well with sceptical guests who do not trust in-house guests' feedback.

Lastly, the most frequent pronoun in the New Zealand dataset is We, which refers to three main groups of people: 1) the hotels, 2) the hosts, 3) the guests. These three referents are an important linguistic resource, which is used to fulfil three of the important steps within Move: (E). 
The first reference of We is the hotels functioning as the subject of verbs that are used to promote the hotels' location, which can be seen in the following examples: "We can be found just 5 minutes from the airport and 10 minutes from the city centre of Rotorua by car" (NZ25). This example functions to demonstrate that the hotel is in a superior location. The second function of We is used to refer to the hosts when they are describing their background and history, which creates a connection and imaginary friendship between the hosts and the readers. It makes the readers feel they know the host before they physically get there. It is a technique to construct a sense of curiosity of the guests' imagination toward the hosts. For example, "We purchased the land in 2003 and spent five years building and developing the site, with Roger doing all the building himself...."(NZ11). In the last example, We refers to the guests who are describing their experience and gratitude toward the hosts. This is an instance of step ig: Introducing guests' feedback. An example is "When We first saw

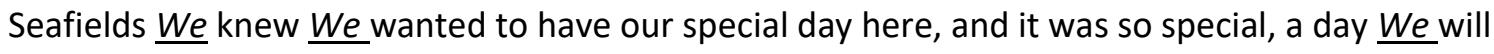
treasure all our lives (NZ2)." Interestingly, this We structure performs a similar function (establishing credibility of the hotels) to Your in the previous section. The occurrence of pronouns We and Your refers to guests who put their feedback in guests' reviews section. Web designers use these two pronouns as a persuasive technique to establish their hotels accreditation to guarantee that their hotels have been certified through the previous guests. This promotional technique is relatively prevalent among tourism and hotel website advertising to provide positive guest feedback as a way to promote their hotels. The hotel owner (T2) said that this technique works more effectively than the in-house promotional texts that the hotels create.

\subsubsection{Move: Describing rooms (DR)}

The next move is Move: Describing rooms (DR), which consists of 24,566 words of the overall datasets. It contains the largest number of words across the five obligatory moves. It is further subdivided into 15,922 words in the Thai dataset and 8,644 in the New Zealand dataset. The primary purpose of this move is to persuade prospective guests to book the accommodation. Motive in this move is to create a desire in readers to stay in the hotels through the promotional descriptions of types of hotel rooms offered, facilities, services, design (theme), atmosphere, outdoor areas, including views of rooms. This move can be realised by the four steps as shown below:

Step df: Describing rooms' facilities/ outdoor areas and views of the rooms

Step ar: Describing atmosphere and design of the rooms (theme of the hotel suites)

Step pr: Stating the price of the rooms for individuals and groups

Step gc: Identifying target groups/capacity of the accommodation 


\section{Verbs in Move: Describing rooms (DR)}

Table 28 reveals a list of frequent verbs and their collocational patterns that are used in Move: (DR) in the Thai and New Zealand hotel datasets. Interestingly, both datasets seem to use two similar verbs (Offer and Equipped) to promote hotel suites, which are related to the main intention of this move. Even though the total number of words in the New Zealand daaset for this move is just over half the total number of words for the equivalent move in the Thai dataset, the difference in the frequency of these two verbs between these two datasets is not significant. Apart from these two verbs, the most frequent verbs whose frequency are not less than 1.5/1,000 words are Designed and Decorated in the Thai dataset and Enjoy in the New Zealand dataset. The collocational behaviours of these verbs are discussed in the next paragraph.

Table 28: Frequency and collocations of verbs in Move: (DR) in the Thai and New Zealand datasets

\begin{tabular}{|c|c|c|c|}
\hline \multicolumn{4}{|c|}{ Thai hotels } \\
\hline Verbs & Collocates & $\begin{array}{l}\text { Per } 1,000 \\
\text { (frequency) }\end{array}$ & $\begin{array}{l}\text { T/BNC } \\
\text { (LL value)* }\end{array}$ \\
\hline Offer & $\begin{array}{l}\text { we/the suites/the } \\
\text { rooms/the room/name of } \\
\text { the hotel +_+ facilities in } \\
\text { the suites/views of the } \\
\text { suites/ accommodation } \\
\text { types/positive qualities of } \\
\text { holiday experience }\end{array}$ & 3.00 & $\begin{array}{l}165.81 \\
p<0.0001\end{array}$ \\
\hline Equipped & _ with facilities in the suites & 2.80 & $\begin{array}{l}376.61 \\
p<0.0001\end{array}$ \\
\hline Designed & $\begin{array}{l}\text { a) verb to be }+ \text { in }+ \text { nouns } \\
\text { (related to decoration } \\
\text { and atmosphere). } \\
\text { b) verb to be }+ \text { + with }+ \\
\text { nouns (related to } \\
\text { decoration and } \\
\text { atmosphere). }\end{array}$ & 2.00 & $\begin{array}{l}131.95 \\
p<0.0001\end{array}$ \\
\hline Decorated & $\begin{array}{l}\text { a)_ with nouns (details of } \\
\text { the furniture } \\
\text { design/material in the } \\
\text { suites } \\
\text { b)_in nouns ( related to a } \\
\text { design of the room) }\end{array}$ & 1.70 & $\begin{array}{l}220.00 \\
p<0.0001\end{array}$ \\
\hline Enjoy & _+noun (facilities/views) & 1.20 & $\begin{array}{l}81.258 \\
p<0.0001\end{array}$ \\
\hline
\end{tabular}

\begin{tabular}{|c|c|c|c|}
\hline \multicolumn{4}{|c|}{ New Zealand hotels } \\
\hline Verbs & Collocates & $\begin{array}{l}\text { Per 1,000 } \\
\text { (frequency) }\end{array}$ & $\begin{array}{l}\text { NZ/BNC } \\
\text { (LL value)* }\end{array}$ \\
\hline Offer & $\begin{array}{l}\text { we/the suites/the } \\
\text { rooms/the } \\
\text { room/name of the } \\
\text { hotel +_+ facilities in } \\
\text { the suites/ views of } \\
\text { the suites/ } \\
\text { accommodation } \\
\text { types/positive } \\
\text { qualities of holiday } \\
\text { experience }\end{array}$ & 4.00 & $\begin{array}{l}140.32 \\
p<0.0001\end{array}$ \\
\hline Enjoy & $\begin{array}{l}\overline{-}^{+} \text {noun } \\
\text { (facilities/views) }\end{array}$ & 2.40 & $\begin{array}{l}112.01 \\
p<0.0001\end{array}$ \\
\hline Equipped & $\begin{array}{l}\text { - with facilities in the } \\
\text { suites }\end{array}$ & 2.10 & $\begin{array}{l}149.83 \\
p<0.0001\end{array}$ \\
\hline Designed & $\begin{array}{l}\text { a) verb to be }+ \text { in }+ \\
\text { nouns (related to } \\
\text { decoration and } \\
\text { atmosphere). }\end{array}$ & 0.90 & $\begin{array}{l}21.52 \\
p<0.0001\end{array}$ \\
\hline Decorated & - & 0.30 & $\begin{array}{l}14.07 \\
p<0.001\end{array}$ \\
\hline
\end{tabular}

*The $p$ value of the comparison of the frequency of verbs (Move: DR) in the Thai dataset and New Zealand dataset with the frequency of the same verbs in the BNC is 0.0001 . It indicates that the difference is highly significant.

The most frequent verbs in the Thai dataset are Offer, Equipped, Designed, and Decorated. In contrast, the most frequent verbs in the New Zealand webpages are Offer, Enjoy, and Equipped. As Offer and Equipped occur in both datasets, in the next paragraph, I discuss them based on 
similarities and differences. Based on the occurrence of frequent verbs in the Thai and New Zealand hotel webpages, it seems to reveal that there are differences in the main focus of Move: (DR) between both hotel webpages. In the Thai dataset, the main focus is on promoting the design and decoration of the suites (theme), while the main intention of hotels in the New Zealand dataset is on presenting facilities and services provided by the hotels. More specifically, Designed and Decorated are frequently used in the Thai datasetin order to describe the suite design, whereas Enjoy is often encountered in the New Zealand dataset in order to describe the facilities and services that the guests can experience.

This section discusses the similarities and differences between the use of common verbs across the two datasets (Thai and New Zealand). Both datasets share two distinctive verbs: Offer and Equipped. Furthermore, these verbs contain a similar set of collocations and they are used frequently in this move in order to promote amenities and views of the accommodation.

Offer is the most frequent verb in both datasets which is used to promote hotel accommodation, which corresponds to the main communicative purpose of Move: (DR). Even though the Thai hotel webpage contains a larger number of words than the New Zealand hotel webpage, the use of offer is more dominant in the New Zealand hotels (4 times per 1,000 words) than the Thai webpages (3 times per 1000 words).

There are five different collocations of Offer (facilities in the suites, views of the suites, accommodation types, and positive qualities of holiday experience) but all of them are related to the description of hotel accommodation. The collocations of Offer serve the primary intention of step: describing rooms' facilities/ outdoor areas and views of the rooms. Offer is followed by five different terms to portray a suite image in the readers' mind in order persuade prospective guests to make a booking. However, only the first two terms are discussed in my analysis. This is because the other three terms do not have enough concordance lines to draw a consistent collocation to analyse.

The most interesting linguistic element that follows offer is offer+ facilities in the suites. It is the most frequent term in Move: Describing rooms (DR). The function of this pattern is simply to describe facilities as can be seen in the following examples: "These studios all offer a kitchenette with tea and coffee making facilities, toaster, fridge and a microwave should you wish to cook" (T5). and “Our Family Suites offer $\mathbf{2}$ queen-size beds plus 1 sofa bed, seating arrangement ..."(NZ7). The other pattern term includes_+views of the suites. For example: "The rooms offer uncompromising views of our courtyard and ornamental waterfall surrounded by a lush tropical garden" (T7). and "Our eight beautifully appointed rooms all offer stunning views over the lake or 
gardens and combine our signature modern styling in a thoroughly contemporary and luxurious English-style manor" (NZ5). To fulfil the purpose of this step, the web designers use this pattern together with evaluative words (uncompromising, stunning, and styling) to create a vision of the described views in the readers' mind. Through the promotional words, the hotels aim to create desire in the potentials guests to book the hotels (Morgan \& Pritchard, 2000). As Morgan and Pritchard (2000) state, audiences make a decision to book the hotels based on mental images that advertisements aim to create (p. 11). This persuasive device is commonly applied in both cultures. It does not only occur in this move. It seems to be the main marketing strategy that is widespread throughout the overall datasets. The hotels draw a picture in the readers' mind of what kind of scenery the guests can see from their room. Generally, rooms with views are more expensive than suites without views. In order to sell this type of room, it is quite important to create a picture of the rooms through promotional materials which include words, pictures, and sounds (Morgan \& Pritchard, 2000, p. 10).

The second most frequent verb that occurs in both the Thai and New Zealand datasets is Equipped. Even though Equipped is more dominant in the Thai hotel webpages $(2.80 / 1,000)$ than the New Zealand webpages $(2.10 / 1,000)$, both datasets share two similar linguistic structures using Equipped. Both structures are related to promoting facilities and amenities in the suite that the hotels provide. The first collocation of equipped is _ with facilities in the suites. In this pattern, Equipped is used only in a passive voice construction to highlight the subject of a sentence. The most frequent nouns that are placed as the subject of this structure are mainly types of suites, for example, villa, studio, and family room. The web designers exploit this structure in order to draw readers' attention to a subject of the sentence, which is the accommodation that the hotel aims to promote. Although the passive voice sounds impersonal and less energetic as suggested by Kline and Hale (2012), I found that the passive voice is a common promotional strategy. It is not the hotel owners' intention to distance themselves from the audience. In fact, the hotel owners aim to put emphasis on the rooms the hotels aim to promote. Equipped, Designed, and Decorated are the verbs that are only used in the passive voice as a marketing strategy to promote the hotels' accommodation.

Designed links to the main intention of Step ar: Describing atmosphere and design of the rooms (theme of the hotel suites) within Move: (DR). Designed is found in both datasets but it is more significant in the Thai dataset. It occurs 2 times per 1,000 words, while it is less than 1 time per 1,000 words in the New Zealand webpages. As the frequency of occurrence of Design is relatively low in the New Zealand dataset, there are only two instances of concordance lines revealing a consistent pattern of collocations of designed. It is verb to be +_in+ nouns (related to 
decoration and atmosphere). Conversely, the concordance lines in the Thai hotel webpages show two distinctive collocational patterns, in which one of them is the same as the New Zealand counterparts. In short, the patterns of collocation of Designed based on the frequency in Move: (DR) are a) verb to be +_in (style of a design of the suites) and b) verb to be +_with + nouns (related to decoration and atmosphere). The first pattern is only found in the Thai dataset and is more prevalent than the second structure, while the second pattern occurs in both datasets. The hotels use these two patterns to describe the design and theme of the rooms. The frequency of Designed in my dataset, where Designed is more significant in the Thai hotels than the New Zealand counterpart, reflects that the Thai hotels aim to focus on the design in order to sell the accommodation. On the other hand, the New Zealand hotels intend to describe the facilities that the guest will have when they stay in the hotels. The following examples illustrate this. "The superior rooms are designed in a Thai style and have marble floors and Baroque style furniture..." (T10), and "Each villa is designed in southern vernacular architecture and is positioned differently to perfectly fit the environment creating unique layouts and pool orientation" (T13). Apart from Designed that the web designers use to create an image of a theme (room) in the readers' mind, other evaluative terms relating to art and decoration also contribute to achieving this intention. For example, Baroque style furniture is a technique used to take the mind of the readers back to the late 16-century where the Baroque architecture began. The hotel uses this Baroque theme to provoke a desire of the readers. Not only do these two examples create an image of a design of the suites in the audience's mind to persuade them to book the hotels, they also tell the readers about the facilities and the view of the suite. More specifically, the second example does not directly tell the readers about what facilities are provided. This is because the hotel focuses on the theme of the room as their main selling point.

The next collocation of Designed is verb to be +_ with + nouns (related to decoration and atmosphere). This structure is less frequent, and there are only few concordance lines compared with the previous pattern that I discussed in the previous section. As a result, I cannot draw out many examples and solid evidence to support my analysis. The examples that support this structure (designed is verb to be +_ with + nouns related to decoration and atmosphere) are "All resort room types are elegantly designed with convenience and relaxing atmosphere in mind (T26)" and "The Pohutukawa room has been designed with romance in mind (NZ19)". These examples follow a similar pattern in order to achieve its function as describing the design of the rooms. They do not explicitly tell the readers about details of decoration and style of furniture. The hotels let the audience imagine further based on what they say, which varies from person to person.

The next frequent verb which is related to describing the theme of accommodation in the Thai hotel webpages is Decorated. This verb occurs only 0.30 times per 1,000 words in the New 
Zealand hotels. Consequently, the concordance lines (NZ dataset) do not allow me to draw a consistent collocation. The collocational patterns that I discuss here are only retrieved from the Thai dataset. There are two patterns: 1) Decorated with nouns (details of the furniture design/material in the suites, and 2) Decorated in nouns (related to a design of the rooms). The primary function of these two patterns is similar to the collocation structure of Designed, where the main intention is to describe a design of the rooms. For example, "The stylish bungalows are decorated with handicraft hardwood floors" and "These luxurious penthouse suites are decorated in Thai style" (T10). Interviews with the boutique hotel owners in Thailand seems to correspond to these examples. The hotel owners say that apart from identifying and being able to respond to the individual needs of a target group, it is important to amplify the hotel's main selling point, which is the design of the accommodation in order to encourage prospective guests to make a booking. The hotel's advertisement intend to differentiate themselves in terms of designs and decorations of the rooms from other hotels, which seems to be one of the most important selling points to attract the readers' attention. This interview data explains why Designed and Decorated are one of the frequent verbs in Move: (DR).

This section discusses the second most frequent verb in the New Zealand hotel webpages. Though Enjoy is not a frequent verb with a frequency less than 1.50 times per 1,000 words) in the Thai dataset, its concordance lines reveal a similar collocation pattern which performs the same function as the New Zealand counterpart. It seems that Enjoy has its own unique pattern and function which play an important role in the hotel webpages. The hotels use enjoy as a persuasive device to describe facilities. Also, Enjoy is used as an invitation to the potential guests to come and have a good time at the hotels. Very often, I observed that the web designers use Enjoy in an imperative structure as an encouragement to persuade readers to book the hotels. Similarly, Janoschka (2004, p. 136), claims that a primary function of imperatives in online promotional material is as explicit activation instructions to motivate the audience to take action of purchase (see more details of imperatives in online advertising in section 6.2.1 chapter 6). In my dataset, enjoy has one distinctive collocation in both datasets, that is Enjoy + noun (facilities/views). For example, "Enjoy uninterrupted views of the Hong Islands from your private pool with Jacuzzi and sun deck" (T18) and "Enjoy plenty of open plan living within the lounge and dining area and outdoor entertaining space on your balcony compliment with modern outdoor furniture" (NZ5). These two examples are of an imperative structure, are used to direct potential guests' attention to the facilities that are provided by the hotels. Enjoy is used to introduce the facilities that are offered by the hotels. The hotels do not only inform the readers of the amenities that the guests can 
experience during their stay, but also, the hotels make a casual invitation to the guests to come to take advantage of the hotel facilities.

\section{Pronouns in Move: Describing rooms (DR)}

Table 29 demonstrates a list of frequent pronouns and their collocational patterns that are used in Move: (DR) in the Thai and New Zealand hotel datasets. Personal pronouns such as You, Your, We and Our are used as a common persuasive strategy in making a sense of solidarity and creating involvement with target guests (Cook, 1992; Fuertes-Olivera, Velasco-Sacristán, ArribasBaño \& Samaniego-Fernández, 2001; Hyland, 2001; Myers, 1994; Pitchinee, 1995 (See more detail of these studies in section 6.2.3 chapter 6). Similarly, in boutique hotel online advertising, I found that personal markers perform the same function in my dataset. The hotels used pronouns to establish a relationship with the target audience. In Move: (DR) the most frequent pronouns: Your, and You are found in both datasets.

Table 29: Frequency and collocations of pronouns in Move: DR in the Thai and New Zealand datasets

\begin{tabular}{|c|c|c|c|}
\hline \multicolumn{4}{|c|}{ Thai hotels } \\
\hline Pronouns & Collocates & $\begin{array}{l}\text { Per } 1,000 \\
\text { (frequency) }\end{array}$ & $\begin{array}{l}\text { T/BNC } \\
\text { (LL value)* }\end{array}$ \\
\hline your & $\begin{array}{l}\text { _noun (types of } \\
\text { accommodation and } \\
\text { its facilities/ } \\
\text { /holiday experience } \\
\text { related) }\end{array}$ & 5.00 & $\begin{array}{l}98.03 \\
p<0.0001\end{array}$ \\
\hline you & $\begin{array}{l}\text { a)_will } \\
\text { find/experience } \\
\text { + noun(facilities and } \\
\text { services) } \\
\text { b)_can+ verb } \\
\text { (holiday experience } \\
\text { related) } \\
\text { +noun (facilities and } \\
\text { services/holiday } \\
\text { experience related }\end{array}$ & 3.10 & $\begin{array}{l}30.20 \\
p<0.0001\end{array}$ \\
\hline
\end{tabular}

\begin{tabular}{|c|c|c|c|}
\hline \multicolumn{4}{|c|}{ New Zealand hotels } \\
\hline Pronouns & Collocates & $\begin{array}{l}\text { Per } 1,000 \\
\text { (frequency) }\end{array}$ & $\begin{array}{l}\text { NZ/BNC } \\
\text { (LL value)* }\end{array}$ \\
\hline your & $\begin{array}{l}\text { a)_noun (types of } \\
\text { accommodation } \\
\text { and its facilities/ } \\
\text { holiday } \\
\text { experience } \\
\text { related) }\end{array}$ & 8.00 & $\begin{array}{l}136.22 \\
p<0.0001\end{array}$ \\
\hline you & $\begin{array}{l}\text { a)_will be able to } \\
\text { + verb (holiday } \\
\text { experience } \\
\text { related) + noun } \\
\text { (facilities and } \\
\text { services) } \\
\text { b)_can } \\
\text { + verb (holiday } \\
\text { experience } \\
\text { related) +noun } \\
\text { (facilities and } \\
\text { services) }\end{array}$ & 6.90 & $\begin{array}{l}101.52 \\
p<0.0001\end{array}$ \\
\hline
\end{tabular}


Table 29 shows the main collocations of pronouns in Move: (DR) in which they are associated with types of accommodation, including facilities and services such as: private suite, studio, bedroom family suite, private pool, spa bath, and lounge bar. Based on a comparison of the frequency of pronouns used in Move: (DR), similar pronouns (your and you,) are found in both Thai and New Zealand hotel webpages even though they exhibit minor differences in terms of their collocational profiles. The following is a discussion of the frequent pronouns and their collocations in comparison of the Thai and New Zealand datasets.

The most frequent pronoun is Your. Web designers use Your to try to convince potential customers that they are an important part of the hotels where they will have their own privacy and indulge themselves in their own sanctuary. Additionally, Your makes the readers feel that their identity and desires are uniquely recognised. Your is the most frequent pronoun used in both datasets. Moreover, the collocations of Your are categorised into two major terms 1)_+noun (types of accommodation and its facilities), and 2)_+ noun (holiday experience related). These two categories both appear in the Thai and New Zealand datasets.

The first category of collocational behaviour of Your is_+ noun (types of accommodation and its facilities). In order to draw the target audience's attention to the suites that the hotel aims to promote, the web designers use Your, which refers to the target guests. This strategy plays with the readers' emotions and feeling. The hotels aim to shape the readers' attitude and make the readers feel connected to the hotel. The web designers intend to convey that their accommodation is uniquely designed for the readers so that they will have full power in their own space. For example, "Your private balcony" (T5) and "your room has modern, inviting décor with a King size bed..."(NZ2). These examples are used to show that the hotels value individual identity and privacy of the guests by the simple use of Your when describing their suites. In addition, the hotels aim to motivate the readers to view their facilities and accommodation as exclusively built for the readers. My analysis seems to be congruent with Morgan and Pritchard's (2000) suggestion. They claim that since the tourism markets have become more sophisticated, in order to win their clients heart, the marketers should emphasise the benefit of the products along with establishing a close relationship with the customer. In the boutique hotel context, the hotels use personal markers as a marketing device to promote their facilities and build engagement with readers.

In regard to the first types of Your collocation:_+ noun (types of accommodation and its facilities), the frequent collocations of Your in the Thai and New Zealand datasets contain certain similar features, for example, room, bedroom stay, private balcony, and spa bath. The hotels are likely to advertise their distinctive amenities to their audience. The hotels have pre-planned that 
these amenities will be able to attract their target groups. Furthermore, there are a few instances showing cultural and geographical differences between the two datasets, including an in room fireplace, infinity-edge private pool, private beachfront, and Jacuzzi. These examples also reflect the life style of local markets and the expectation of tourists. In Thailand, having a private pool signifies a luxurious lifestyle; most boutique hotels aim to upscale their hotels by advertising these expensive amenities. For example, "Step outside onto a spacious sundeck and plunge into your refreshing 5.5x3.5 meters infinity-edge private pool..."(T 12). Infinity-edge private pool seems to signal that the hotels are exclusively for guests who would like to experience a sophisticated lifestyle. Likewise, an in room fireplace reflects characteristics and atmosphere of the New Zealand boutique hotels in my dataset. It is associated with warmth and a homely atmosphere.

Additionally, there are a few accommodation type terms that only occur in the New Zealan ddataset, which include unit, studio, lodge, and apartment. These terms appear to reflect the unique characteristics of New Zealand accommodation and leisure patterns. According to Morgan, Pritchard, and Pride (2002), New Zealand's 100\% pure campaign is the first international campaign which was designed to boost income for the country. This programme focuses on backpackers and thrill seeker paradise who wants to explore natural environment destination (p. 285). It seems that these accommodation terms (unit, studio, lodge, and apartment) match with the Kiwi tourism trend, which focuses on an adventure theme. However, these terms do not exist in the Thai hotel webpages. The terms that are commonly used to refer to Thai boutique hotels are villa, bungalow and resort. This could be explained by the expectation of tourists, including leisure patterns in New Zealand, are different from Thailand.

Another interesting aspect of the first collocational pattern of Your $L+$ noun types of accommodation and its facilities) is that this pattern frequently co-occurs with own. This structure occurs in the New Zealand boutique hotel websites more frequently than in the Thailand hotels. In this category, own is exploited to emphasise the exclusive privacy of target guests when it is used together with Your. For example, "Relax in your own spa bath (T9)." Due to the fact that the use of own in the New Zealand dataset is more prevalent than in the Thai dataset; it is interesting to explore the factors behind the difference. My proposed explanation for this occurrence is because in Thailand own is one of the unmarked grammatical features that may not be formally taught in schools. In addition, there is no parallel concept or term referring to Own in Thai. Consequently, Own is not commonly found in many of Thai people's lexical repertoire. Another explanation is that it may be due to cultural differences. New Zealand society is individualistic and highly values freedom and personal space (www.tourism.net.nz). The higher frequency of Own in the New Zealand dataset compared to the Thai dataset may reflect the individualism in New Zealand culture. 
The second category of collocation of Your is _ +noun (holiday experience-related) aims to construe emotional reactions from the readers by using evaluative language, for example, memorable, relaxing, and romantic. This pattern is more common in the Thai hotel webpages than the New Zealand webpages. It implies that the Thai hotels tend to use more evaluative language to trigger emotions from the audience as a device to persuade them to book the hotels than the New Zealand hotels. This communicative purpose may be accomplished by the use of adjectives plus holiday experience. The adjectives are used to play to the imagination of the readers and build a picture of them enjoying holidays at the hotels. For example, "The hotel's serene ambience, together with spectacular sea view and pristine landscape, ensures an ideal romantic getaway and your memorable seaside vacation" (T1). This example supports my suggestion that the Thai hotels employ evaluative language in order to create desire from audience. In the New Zealand webpages, web designers tend to be more descriptive; the hotels mainly describe facilities in the suites in order to achieve their intent. It shows the New Zealand websites contains less evaluative language as in the following example: "Your unit has an LCD TV (NZ21)."

In addition to the pronoun Your, You also exists in Move: (DR). In order to achieve the main purpose of this move (promote the hotel accommodation and persuade the readers to take an action of purchase), the promotional materials have to be clear to whom messages are addressed. In my dataset, You refers to target guests and it is used as the subject of a sentence to show that you performs actions in the sentence. In the context of boutique hotels, the characteristics of You as a target group is constructed by the use of nouns and evaluative adjectives in the hotels promotional materials. In hotel accommodation and amenities online advertisements, the identity of You can be illustrated as a group of people who are seeking a unique, luxurious and stylish holiday experience, but they still expect to have their own personal privacy. This interpretation is supported by the frequent use of private, unique, and luxurious. The target market of the boutique hotels may be business travellers, families on vacation or couples on honeymoon trips who love outdoor activities, for example, sunbathing or reading books on a private deck, indulging themselves in a private pool. Owing to the high frequency of $\mathrm{Wi}-\mathrm{Fi}$ in the dataset, it suggests that the target guests and their lifestyle are likely to be business travellers or a group of people who do not want to miss a communication and use the Internet during their trip.

\subsubsection{Move: Introducing restaurants/bars, lounges, and libraries (R)}

The last move that I will discuss is Move: (R); it contains 8,742 words of the overall dataset, 5,494 words in the Thai dataset and 3,248 in the New Zealand dataset respectively. Even though Move: $(R)$ is not an obligatory move in my dataset, because it occurs less than $75 \%$ in both 
dataset, after I examined its linguistic features, I found very interesting features that specifically occur in this move. Therefore, I also included move: $(R)$ in my register analysis. The purpose of this move is to describe the services, facilities, design and atmosphere of the restaurants, bars, lounges, and libraries which are offered by the hotels. This move can be achieved by three distinct steps: Step fr: Specifying facilities and services of the restaurants, bars, lounges, and libraries Step ds: Describing design, exterior scenery, and atmosphere of the restaurants, bars, lounges, and libraries.

Step fd: Providing information about food and drinks

\section{Verbs in Move: Introducing restaurants/bars, lounges, and libraries}

Table 30 shows the list of frequent verbs and their collocational profiles in Move: (R). Comparing occurrence of verbs, Enjoy and Serve are commonly found in both the Thai and New Zealand datasets; however, their collocational structures show a slight difference. Thai boutique hotel texts use four frequent verbs in order to achieve the communicative purpose of Move: (R) (Enjoy, Offer, Located and Serve) compared with three instances of verbs (Serve, Enjoy, and Offer) that appear in the New Zealand hotel advertising texts.

Table 30: Frequency and collocations of verbs in Move: $(R)$ in the Thai and New Zealand datasets

\begin{tabular}{|l|l|l|l|}
\hline \multicolumn{4}{|c|}{ Thai hotels } \\
\hline Verbs & Collocates & $\begin{array}{l}\text { Per 1,000 } \\
\text { (frequency) }\end{array}$ & $\begin{array}{l}\text { T/BNC } \\
\text { (LL value) }\end{array}$ \\
\hline enjoy & $\begin{array}{l}\text { a)_+ restaurant facilities } \\
\text { and services } \\
\text { B) guest+ can +_+ } \\
\text { restaurant facilities and } \\
\text { services }\end{array}$ & 3.40 & $\begin{array}{l}114.47 \\
p<0.0001\end{array}$ \\
\hline offer & $\begin{array}{l}\text { offer + restaurant } \\
\text { facilities and services }\end{array}$ & 2.10 & $\begin{array}{l}41.32 \\
p<0.0001\end{array}$ \\
\hline located & $\begin{array}{l}\text { Restaurants + verb to } \\
\text { be+_+ on + location }\end{array}$ & 2.00 & $\begin{array}{l}75 \\
p<0.0001\end{array}$ \\
\hline serve & - ${ }^{\text {food }}$ & 1.80 & $\begin{array}{l}51.49 \\
p<0.0001\end{array}$ \\
\hline
\end{tabular}

\begin{tabular}{|l|l|l|l|}
\hline \multicolumn{5}{|c|}{ New Zealand hotels } \\
\hline Verbs & \multicolumn{1}{|c|}{ Collocates } & $\begin{array}{l}\text { Per 1,000 } \\
\text { (frequency) }\end{array}$ & $\begin{array}{l}\text { NZ/BNC } \\
\text { (LL value) }\end{array}$ \\
\hline & $\begin{array}{l}\text { food+ verb to be + } \\
\text { serve } \\
\text { in + location }\end{array}$ & 9.20 & $\begin{array}{l}250.41 \\
\mathrm{p}<0.0001\end{array}$ \\
\hline enjoy & $\begin{array}{l}\text { + restaurant } \\
\text { facilities and } \\
\text { services }\end{array}$ & 4.00 & $\begin{array}{l}41.32 \\
\mathrm{p}<0.0001\end{array}$ \\
\hline offer & $\begin{array}{l}\text { Restaurants +_+ } \\
\text { restaurant facilities } \\
\text { and services }\end{array}$ & 2.70 & $\begin{array}{l}35.01 \\
\mathrm{p}<0.0001\end{array}$ \\
\hline \multicolumn{3}{|l}{} \\
\hline
\end{tabular}

*The $p$ value of the comparison of the frequency of the verbs (Move: $\mathrm{R}$ ) in the Thai and New Zealand datasets with the frequency of the same verbs in the BNC is $<0.0001$. It indicates that the difference is highly significant.

Table 32 reveals the similarities and differences between the use of common verbs across the two datasets. Both datasets share three frequent verbs: (Enjoy, Serve, and Offer). However, these verbs contain a different set of collocations in the two datasets. Only Offer shares similar collocations in both datasets. 
The most frequent verb that appears in the Thai dataset is Enjoy with 3.4 frequency of occurrence/1,000 words; it also occurs as the second most frequent in the New Zealand dataset whose demonstrated frequency is $4 / 1,000$ words. In the Thai hotels, the data reveals two collocational patterns: 1) _+ restaurant facilities and services, 2) guest+ can+_+ restaurant facilities and services. In contrast, in the New Zealand dataset, only the first collocation of Enjoy occurs. The first structure is used in the imperative but implies an invitation not a command, as can be seen in this example: "Enjoy cocktails, local and international beers and wines, and specialty coffees from our expert barista and mixologists" (NZ20). Enjoy in the imperative is used as an advertising technique designed to offer a casual invitation from the hotels to their target guests in order to persuade them to experience the services, facilities, and activities that the hotel restaurant offer. In addition to introducing restaurant services, Enjoy in the imperative is also used to encourage guests to imagine themselves indulging in the restaurant atmosphere and surrounding environment. An example is, "Enjoy spectacular views of the surrounding islands while dining on fresh local seafood and authentic Italian pizzas..."(T13). This example reflects the communicative purposes of the step: describing design, exterior scenery, and atmosphere of the restaurants, bars, lounges, and libraries and step: providing information about food and drinks which are used to achieve the advertising impact of Move: (R). The second collocation of Enjoy is guest + can +_ + restaurant facilities and services. This structure is mainly encountered in the Thai dataset. Even though the structure in which Enjoy is used is different, its main purpose remains the same; namely, to inform the readers about facilities and services and the surrounding views of the restaurants. For example, "...guests can enjoy the innovative cuisine in a European influenced bistro setting for breakfast, lunch and dinner (T25)." This example shows the visitors what kind of food is served and when. Can in this collocational structure is used to express the opportunity that guests can enjoy and experience restaurant services. Can in hotel promotional context is commonly adopted as a persuasive device or invitation to motivate the guests to book the hotels, rather than as a method to describe ability or to offer permission.

In contrast, the most frequent verb in the New Zealand hotel webpage is Serve which occurs 9.2/1,000 words compared to $1.8 / 1,000$ words in the Thai dataset, representing the fourth most frequent verb. Although Serve is usually encountered in both datasets, the way the word Serve is adopted by the web designers to describe restaurant services is different. In the New Zealand dataset, the pattern is food+verb to be+_(past participle) + in + location as can be seen in this example: "Breakfast is served in our upstairs dining room..."(NZ6). In this pattern Serve is mainly used in the passive form to inform the readers about where the breakfast is provided. This structure tells the web audience about what is served and where. On the contrary, the Thai hotel websites use 
Serve in the active form, as can be seen in this example: "Our restaurant serves traditional authentic Thai cuisine...(T14)." On the Thai boutique hotel websites Serve is used only in the active form to inform the readers about the restaurant services. The differences in how Serve is used may be caused by the fact that the both datasets have different foci when describing restaurant services. It seems that in the Thai dataset, the main focus is on the hotels themselves who offer services, while in the New Zealand dataset the main emphasis is on what food is being served.

The second most frequent verb in the Thai dataset is Offer, representing 2.1 occurrence/1,000 words. In regard to the collocational profile of the word Offer, the structure is restaurants +_ + restaurant facilities and services. An example is, “...our poolside bar offers light snacks, alcoholic beverages and cocktails..." $(T)$. This collocation is connected to the main communicative function of step: specifying facilities and services of the restaurants, bars, lounges, and libraries.

Lastly, Located has 1.8 occurrence/1,000 words. Although Located is not the most frequent verb in the Thai dataset, it is prevalent compared to the BNC corpus. Located is used in Move: $(R)$ in order to describe the location of the restaurant and bars as can be seen in this phrase: "The restaurant is located on the beachfront at Bo Phut" (T12). However, there are some instances of Located in which verb to be is omitted when introducing the location of the hotel restaurants, as can be seen in the following examples, "Di Mare has a relaxed ambiance in a beautiful tropical setting featuring an open deck adjacent to a delightful lotus pond and located only metres..." (T13)and "Our open-air Restaurant located on the level of MUTHI MAYAs Lobby..."(T9). Interestingly, it is a stylistic technique that is used to describe the location of the restaurants in the Thai boutique hotel websites.

\section{Adjectives in Move: Introducing restaurants/bars, lounges, and libraries}

Table 31 reveals that, in the Thai dataset, the eleven most frequent adjectives are Thai, Traditional, Available, Traditional, Special, Romantic, Fresh, Private, Local, Daily, International, and Delicious. In contrast, in the New Zealand dataset, the adjectives that commonly occur are Dining, Cooked, Fresh, Available, Local, Seasonal, Complimentary, Special, Complimentary, Baked, Free, and Cosy. 
Table 31: Frequency of adjectives and collocations in Move: $(R)$ in the Thai and New Zealand datasets

\begin{tabular}{|c|c|c|c|}
\hline \multicolumn{4}{|c|}{ Thai hotels } \\
\hline Adjectives & Collocates & $\begin{array}{c}\text { Per } 1,000 \\
\text { (frequency) }\end{array}$ & $\begin{array}{l}\text { T/BNC } \\
\text { (LL- } \\
\text { value)* }\end{array}$ \\
\hline Thai & $\begin{array}{l}\text { + nouns } \\
\text { (food/cuisine/dishes/ } \\
\text { menu/desserts) }\end{array}$ & 9.00 & $\begin{array}{l}665.70 \\
p<0.0001\end{array}$ \\
\hline Available & $\begin{array}{l}\text { a) noun (food and } \\
\text { restaurant services) }{ }_{+}- \\
\text {b) nouns+ verb to be+ } \\
\text { available }\end{array}$ & 4.10 & $\begin{array}{l}67.46 \\
p<0.0001\end{array}$ \\
\hline Traditional & $\begin{array}{l}\text { + Thai } \\
\text { dishes/cuisine/recipes/ } \\
\text { menus }\end{array}$ & 3.00 & $\begin{array}{l}85.11 \\
p<0.0001\end{array}$ \\
\hline Special & $\begin{array}{l}\text { +menu/prices/ } \\
\text { occasion }\end{array}$ & 2.50 & $\begin{array}{l}43.55 \\
p<0.0001\end{array}$ \\
\hline Romantic & - dinner/set up & 2.10 & $\begin{array}{l}79.99 \\
p<0.0001\end{array}$ \\
\hline Fresh & $\begin{array}{l}+ \text { nouns } \\
\text { (food/fruits/vegetables/ } \\
\text { drinks) }\end{array}$ & 2.10 & $\begin{array}{l}54.05 \\
p<0.0001\end{array}$ \\
\hline Private & _+parties & 2.10 & $\begin{array}{l}33.72 \\
p<0.0001\end{array}$ \\
\hline Local & _tseafood/cuisine & 2.10 & $\begin{array}{l}18.63 \\
p<0.0001\end{array}$ \\
\hline Daily & serves/opens +_ & 1.80 & $\begin{array}{l}44.84 \\
p<0.0001\end{array}$ \\
\hline International & _+cuisine/food/ dishes & 1.80 & $\begin{array}{l}24.93 \\
p<0.0001\end{array}$ \\
\hline Delicious & _+ food/dishes & 1.60 & $\begin{array}{l}50.40 \\
p<0.0001\end{array}$ \\
\hline
\end{tabular}

\begin{tabular}{|c|c|c|c|}
\hline \multicolumn{4}{|c|}{ New Zealand hotels } \\
\hline Adjectives & Collocates & $\begin{array}{l}\text { Per } 1,000 \\
\text { (frequency) }\end{array}$ & $\begin{array}{l}\text { NZ/BNC } \\
\text { (LL - } \\
\text { value)* }\end{array}$ \\
\hline Dining & $\begin{array}{l}\text { + nouns (room, } \\
\text { environments, and } \\
\text { table) }\end{array}$ & 4.90 & $\begin{array}{l}150 \\
p<0.0001\end{array}$ \\
\hline Cooked & $\begin{array}{l}\text { home-cooked/home } \\
\text { cooked }\end{array}$ & 4.60 & $\begin{array}{l}147.53 \\
p<0.0001\end{array}$ \\
\hline Fresh & $\begin{array}{l}{ }_{-}^{+} \text {nouns (food/fruits/ } \\
\text { vegetables/drinks) }\end{array}$ & 4.60 & $\begin{array}{l}98.21 \\
p<0.0001\end{array}$ \\
\hline Available & $\begin{array}{l}\text { A) noun (food and } \\
\text { restaurant services) } \\
++ \\
\text { B) nouns+ verb to be } \\
+{ }_{+}\end{array}$ & 4.00 & $\begin{array}{l}46.14 \\
p<0.0001\end{array}$ \\
\hline Local & $\begin{array}{l}\text { + restaurants/ } \\
\text { wine }\end{array}$ & 3.30 & $\begin{array}{l}25.09 \\
p<0.0001\end{array}$ \\
\hline Seasonal & $\begin{array}{l}\text { + fruits/ } \\
\text { cuisine }\end{array}$ & 2.70 & $\begin{array}{l}84.65 \\
p<0.0001\end{array}$ \\
\hline Special & _+ occasion & 2.70 & $\begin{array}{l}29.83 \\
p<0.0001\end{array}$ \\
\hline $\begin{array}{l}\text { Compliment } \\
\text {-ary }\end{array}$ & _+drinks & 2.40 & $\begin{array}{l}90 \\
p<0.0001\end{array}$ \\
\hline Baked & ${ }_{-}+$(bakery products) & 1.80 & $\begin{array}{l}55.89 \\
p<0.0001\end{array}$ \\
\hline Free & _+ range eggs & 1.80 & $\begin{array}{l}15.92 \\
p<0.0001\end{array}$ \\
\hline Cosy & _+couches/atmosphere & 1.50 & $\begin{array}{l}44.48 \\
p<0.0001\end{array}$ \\
\hline
\end{tabular}

*The $p$ value of the comparison of the frequency of the adjectives (Move: $\mathrm{R}$ ) in the Thai and New Zealand datasets with the frequency of the same adjectives in the BNC is $<0.0001$. It indicates that the difference is highly significant.

Table 32 shows similarities and differences between the use of adjectives across the two datasets (Thai and New Zealand). Both datasets share four similar adjectives: Available, Special, Fresh, and Local. In addition, these adjectives have relatively similar collocational patterns, except for local that presents different collocations. The Thai dataset uses local + seafood/cuisine, whereas local+ restaurants/wine is used in the New Zealand dataset. These differences may be the result of their respective local specialties. The South of Thailand is a rich and popular resource for seafood, while New Zealand wines are well-recognised. 
Table 32: Frequency and collocations of similar adjectives in Thai and New Zealan datasets

\begin{tabular}{|c|c|c|c|c|}
\hline \multicolumn{5}{|c|}{ Similar adjectives in Thai/New Zealand datasets } \\
\hline Adjectives & Collocates & $\begin{array}{c}\text { Thai } \\
\text { Per } 1,000 \\
\text { (frequency) }\end{array}$ & \begin{tabular}{|l|} 
NZ \\
Per 1,000 \\
(frequency)
\end{tabular} & $\begin{array}{l}\mathrm{T} / \mathrm{NZ} \\
\text { (LL value)* }\end{array}$ \\
\hline Available & $\begin{array}{l}\text { a) noun (food and restaurant } \\
\text { services) }{ }^{+}- \\
\text {b) nouns+ verb to be+ available. }\end{array}$ & 4.10 & 4.00 & $\begin{array}{l}0.07 \\
p<0.05\end{array}$ \\
\hline Special & -+menu/prices/ occasion & 2.50 & 2.70 & $\begin{array}{c}0.04 \\
p<0.05\end{array}$ \\
\hline Fresh & $\begin{array}{l}\text { +nouns (food/fruits/vegetables/ } \\
\text { drinks) }\end{array}$ & 2.10 & 4.60 & $\begin{array}{c}4.5 \\
p<0.05\end{array}$ \\
\hline Local & $\begin{array}{l}\text { collocation in the Thai hotels _ } \\
+ \text { seafood/cuisine collocation } \\
\text { in the New Zealand hotels } \\
\text { _+restaurants/ wine }\end{array}$ & 2.10 & 3.30 & $\begin{array}{c}1.09 \\
p<0.05\end{array}$ \\
\hline
\end{tabular}

*P value of a comparison of the adjectives (Move: $R$ ) in the Thai dataset with the same adjectives in New Zealand dataset is $p<0.05$. It indicates that the difference is not significant.

The adjective common to both datasets, fresh has 2.1 occurrences/1,000 words in the Thai hotel webpages compared to 4.6 frequency of occurrences/1,000 words in the New Zealand hotel webpage. It implies that the use of Fresh in the New Zealand dataset is distinctive compared to the Thai dataset. The collocational profile of Fresh is similar across the two datasets, which is Fresh + nouns (food/fruits/vegetables/drinks). Examples are "Special Egg Whisk- Eggs scrambles with corn and fresh chives, nestled on full grain toast (NZ7)" and "We serve premium quality food, fresh seasonal vegetables and juice...(T1)." The function of this structure is connected to one of the steps within Move: (R), which is step: providing information about food and drinks. The main communicative purpose of this step is to highlight a wide range of food, drinks, and ingredients that the hotels offer to the guests. The hotels use Fresh to promote the food that is offered in the menus. The main emphasis is the freshness of natural ingredients and flavor of the food. In particular, hotels and restaurants are recognising that there is a new trend of diet and nutrients, with people increasingly focusing on their health. They avoid processed food and choose to consume only fresh food. This might be one possible reason that the hotels use the adjective Fresh to advertise their restaurant.

Although Fresh is common, as indicated, the most frequent adjective in the Thai dataset is Thai which is unsurprising because the purpose of Move: (R) is to advertise food and drinks including restaurant services. It denotes the unique way that Thai is used to promote food/ restaurant facilities and services. This adjective commonly collocates with the following terms food, cuisine, dishes, 
menu, and desserts, as can be seen in this example: "Our restaurant serves traditional authentic $\underline{T h a i}$ cuisine and is justly famous for high quality" (T4). Thai cuisine is used to promote as a key selling point to travellers who would like to experience real Thai dishes when they visit Thailand.

In contrast, the most frequent adjective in the New Zealand dataset is Dining whose demonstrated frequency is 4.9/1,000 words. The collocations of Dining are + (nouns) room, environment, and table. This pattern is used to perform two different functions. The first function is to inform the readers about the restaurant service hours and location, which can be seen in this example, "Breakfast is served in our upstairs dining room from 7am to $9.30 \mathrm{am} . . . "$ (NZ6) This collocational pattern is likely to be related to breakfast serving time and its location. The hotels generally provide complimentary breakfast for guests; therefore, the information regarding service hours for breakfast and its location is crucial. The second purpose of the collocation of Dining is to describe the ambience of restaurants, bars and surrounding environment to create the desire in guests to use restaurant services. This function is connected to the purpose of step: describing design, exterior scenery, and atmosphere of the restaurants, bars, lounges, and libraries. An example is "Enjoy alfresco in summer or in our bright and cheerful dining room all year round" (N14). This example shows that the web designers urge the guests to imagine or build a picture of themselves indulging in the restaurant atmosphere through the use of evaluative words: bright and cheerful.

The next adjective in the Thai datadet that I discuss here is Traditional, which has 3 occurrences/1,000 words. Traditional commonly collocates with cuisine, dishes, recipe, and methods. The main purpose of this pattern is to introduce Thai foods that are conventionally prepared and cooked with authentic and local ingredients by the chefs who have expertise in Thai food. For example, "At U Chiang Mai you will experience local cuisine cooked using traditional methods and served in the finest tradition of Thai hospitality" (T25). The hotels use Traditional to emphasise the value of originality in the taste and the method of cooking.

The second most frequent adjective in the New Zealand hotel webpage is Cooked which has 4.6 occurrences $/ 1,000$ words. This frequency shows that Cooked is a prevalent term in boutique hotel websites in New Zealand. Its common collocation is home as can be seen in the following structure: home+_. The purpose of this structure is to promote the authenticity of kiwi hospitality and food which is prepared at home and cooked by hosts with local products. For example, "our sumptuous breakfasts are prepared with fresh, produce from our garden and home-cooked dinners are available by arrangement" (NZ17). Home-cooked in this move emerges into two different variants: Home-cooked and Home cooked. However, Home-cooked is a more common form because it occurred five times compared to Home cooked that occurred only once. In addition, in the BNC, 
Home-cooked appears five times ( 35 hits), more than Home cooked ( 7 hits). These two variants are merged into one form, Home-cooked, which occurs more frequently than its counterpart.

In regard to the cultural implications of Home-cooked, the term seems to reflect one of the characteristics of boutique hotels in New Zealand, that they are family-owned businesses. The hosts are responsible for cooking and taking care of the visitors. Moreover, web designers use Homecooked to construct a friendly relationship and cozy ambience between the readers and the hotels. An example is, "For a special occasion or just for your convenience choose to have a delicious home cooked meal with Janet and lan" (N11). This example illustrates how the hosts construct a warm atmosphere and casual invitation by asking the guests to enjoy a Home-cooked meal. It is common for New Zealand culture to welcome guests with their Home-cooked meal; they do not normally take the guests out for dinner, while in the Thai dataset, no Home-cooked is found. In Thai culture, it is very common for the hosts to take the guests to a restaurant for dinner. The New Zealand web designers exploit the word Home-cooked as an advertising tool to catch the attention of the readers who would like to experience real Kiwi culture and authentic food.

The next adjective in the Thai dataset is Romantic whose frequency is $2.1 / 1,000$ words. Romantic usually co-occurs with set up/dinner/view with the main purpose to promote the location and atmosphere of restaurants, bars, including the surrounding views. An example is, "And a romantic dinner with fullmoon light on the sea with aroma candle..."(T27) The word are used to create emotional appeal and to play to the imagination of the visitors about the atmosphere and setting of the restaurants and bars. The hotels use the word Romantic to target couples who would like to experience romance or celebrate their special occasions.

The next frequent adjective in the Thai dataset is Available, with 4.1/1,000 occurrence. It performs two functions which are related to two of the steps within this move. They are step: specifying facilities and services of the restaurants, bars, lounges, and Libraries and step: providing information about food and drinks. Even though Available is not categorised in the five most frequent words in the New Zealand dataset, it occurs as frequently as those in the Thai dataset, at 4/1,000 words. It implies that they are similar in terms of the frequency of Available between the two datasets. In addition to the comparable frequency of Available between the Thai dataset and New Zealand dataset, their collocations and functions are also quite similar. The first structure is noun (food and restaurant services) + _. In this context, Available is used to modify nouns when describing restaurant services that are available to the guests as can be seen in this example, "International and other Asian menu also available" (T12). Although this function of Available is found in both datasets, it is much more common in the Thai hotels. These differences can be explained by the fact that the Thai hotel promotional texts usually use short phrases presented in 
a bullet point giving the lists of facilities and services in the restaurants, so the omission of verb to be is applied to create advertising impact as quickly as possible. However, the New Zealand websites tend to present their information in full sentence.

The second collocation of Available is nouns+ verb to be+ Available. This pattern also has the same purpose as the first pattern, which is to describe the services and facilities that are available in the restaurants. However, there are slight differences in terms of the detail of the description of the services between these two structures. Whereas the first structure describes what kind of services the hotels have, the second structure gives more detail about when and where they can be found. An example is, "Refreshments are available from the lobby bar" (T8). The second structure usually collocates with descriptions of services hours, location of the services.

\section{Pronouns in Move: Introducing restaurants/bars, lounges, and libraries}

Table 33 demonstrates the frequency and collocational behaviours of the frequent pronouns in Move: (R). Based on a comparison of the prevalence of pronouns used in this move, similar sets of pronouns (Our, Your, You, and We) are found in both the Thailand and the New Zealand datasets even though they exhibit minor differences with respect to their frequency and collocations.

Table 33: Frequency of pronouns and collocations in Move (R) in the Thailand and New Zealand datasets

\begin{tabular}{|c|c|c|c|}
\hline \multicolumn{4}{|c|}{ Thai hotels } \\
\hline Verbs & Collocates & $\begin{array}{l}\text { Per } 1,000 \\
\text { (frequency) }\end{array}$ & $\begin{array}{l}\text { T/BNC } \\
\text { (LL value)* }\end{array}$ \\
\hline Our & $\begin{array}{l}\text { + } \\
\text { and drinkstaurants/bars/ hotel staff }\end{array}$ & 9.60 & $\begin{array}{l}152.85 \\
p<0.0001\end{array}$ \\
\hline You & $\begin{array}{l}\text { a) hotels/ restaurants + } \\
\text { verbs (offering/providing/ } \\
\text { serving ) +_+ services } \\
\text { b)_+ verb (experiential } \\
\text { verbs) + restaurant services } \\
\text { and facilities }\end{array}$ & 9.20 & $\begin{array}{l}5.30 \\
p<0.05\end{array}$ \\
\hline Your & $\begin{array}{l}\text { A) _+ dietary needs, tastes, } \\
\text { taste buds, and preference. } \\
\text { B) _ + hotel staff }\end{array}$ & 7.20 & $\begin{array}{l}70.82 \\
p<0.0001\end{array}$ \\
\hline We & $\begin{array}{l}\text { _+ verbs (offering/providing } \\
\text { services) }\end{array}$ & 3.80 & $\begin{array}{l}0.18 \\
p>0.05\end{array}$ \\
\hline
\end{tabular}

\begin{tabular}{|c|c|c|c|}
\hline \multicolumn{4}{|c|}{ New Zealand hotels } \\
\hline Verbs & Collocates & $\begin{array}{l}\text { Per 1,000 } \\
\text { (frequency) }\end{array}$ & $\begin{array}{l}\text { NZ/BNC } \\
\text { (LL value)* }\end{array}$ \\
\hline Our & $\begin{array}{l}\text { + restaurants/bars/food } \\
\text { and drinks/ hotel staff }\end{array}$ & 11.00 & $\begin{array}{l}122.98 \\
p<0.0001\end{array}$ \\
\hline We & $\begin{array}{l}+ \text { verbs (offering/providing } \\
\text { services) }\end{array}$ & 6.70 & $\begin{array}{l}7.93 \\
p<0.01\end{array}$ \\
\hline Your & - hosts & 4.60 & $\begin{array}{l}16.06 \\
p<0.0001\end{array}$ \\
\hline You & $\begin{array}{l}\text { hotels/ restaurants + verbs } \\
\text { (offering/providing/serving) } \\
+\ldots+\text { services }\end{array}$ & 3.07 & $\begin{array}{l}7.73 \\
p<0.01\end{array}$ \\
\hline
\end{tabular}

*The $p$ value of the comparison of the frequency of the pronouns (Move: R) in the Thai and New Zealand datasets with the frequency of the same pronouns in the BNC is between $p>0.05$ and $p<0.0001$. 
The most frequent pronoun in the Thai and New Zealand datasets is Our; it accounts for 9.6 and 11 occurrences/1,000 words respectively. In the context of boutique hotel online advertising, Our is used to signify the ownership of the hotels, which mainly refers to "the hotels", "hotel staff" and "their facilities and services". In regard to the collocational profiles of Our, both the Thai and New Zealand datasets exhibit similar profiles in which they are categorised into three different categories:1)_ +restaurants/bars, 2)_ +food and drinks, 3) _ + hotel staff. Furthermore, these three collocations are connected to the main communicative purpose of Move: $(\mathrm{R})$ namely, to promote restaurants/bars, lounges, and libraries services and facilities.

The first collocation of Our is_trestaurants/bars as can be seen in the following examples: “our poolside bar" (T15), “our Eat restaurant" (T4), "our upstairs dining room" (N6Z), and "our bright and cheerful dining room" (NZ20). The function of this collocation is to introduce the restaurant and inform the readers about where the restaurant services are provided. Also, this collocational pattern is associated with cultural differences between the Thai and New Zealand dataset. As Dining room does not appear in the Thai hotel dataset, it may be referred that dining room is not a common concept which is used to promote hotel restaurant facilities and services. Dining room in the Thai context generally refers to the area in the houses where family members gather for meals. In contrast, the term Dining room in the New Zealand hotel webpages is more commonly used to refer to dining areas where hotels set up for guests to have meals. This reflects the characteristics of boutique hotels in New Zealand, in which the hotel owners transform their private properties into small scale boutique accommodation.

The second collocational pattern of Our is_+ food and drinks as in the following examples: “our sea breeze afternoon tea set" (T12), "our splendid foods and drinks from buffet lines"(T13), "our delicious tapas menu (NZ13)," and our famous pancakes (NZ20)." This pattern is used to promote the food and drinks that are provided by the hotels. Web designers typically use attributive adjectives: splendid, delicious, famous to modify their hotel services in order to create emotional appeal for the readers and persuade them to come and use the restaurant services.

The last collocational structure of Our is _ + hotel staff which can be seen in these examples: "our expert baristas and mixologists (T20), "our formally dressed service staff" (T8), and "our professional chef (T14)." This collocation is adopted not only to promote the hotel services but also to construct the credibility of the hotels by having experts who specialise in cooking and providing good service.

The second pronoun which is frequently encountered in the Thai and New Zealand datasets is Your. It occurs 7.2/1,000 words in the Thai dataset compared to 4.6/1,000 words in the New Zealand dataset. In Move: (R), Your refers to the ownership of the guests, and it suggests that 
the guests can have access to use facilities and services offered by the hotels. Even though, Your is commonly found in both hotel webpages, their collocational patterns reveal certain differences. Thai boutique hotel websites use Your into two different patterns: 1)_+ dietary needs, tastes, taste buds, and preference. 2) _ + hotel staff. In contrast, in the New Zealand dataset, there is only one common pattern; namely, _ + hosts. Starting with main collocations of Your in the Thai dataset_+ dietary needs, tastes, taste buds, and preference, are illustrated in the following examples: “...Fai Restaurant will satisfy your taste buds" (T17). In this pattern Your does not refer to the ownership provided (by the hotels) to the guests when using facilities and services which is previously discussed. Your, however, refers to individual desire or personal preference that the guests have, which are recognised by the hotels. The hoteliers adopt this pattern to highlight that the hotels pay attention to every detail to make their guests happy and they are capable of catering for guests' individual desires for food. The second pattern of Your is_+ hotel staff, which can be seen in these instances: "Please contact your e-concierge to reserve with best view" (T12) and "...in the very private Thai sala on the beach with your very own butler" (T12). These examples reflect the large scale of boutique accommodation in Thailand. Interestingly, it is also used to show that these hotels are happy to provide ownership to the guests to the extent of having personalised services.

In the New Zealand dataset, the main collocational pattern of Your is _ + hosts. Examples are, "...an invitation to join your hosts for a casual evening meal" (NZ19) and ... "begins with complimentary evening drinks with your kiwi hosts...(NZ19)." These two examples do not signify the ownership of the guests to have personalised services but rather to construct a friendly relationship between the readers and the hosts.

The next common pronoun in the Thai and in the New Zealand datasets is You, it accounts for 9.2/1,000 words and 3.07/1,000 words respectively. Even though the Thai and New Zealand datasets are different in terms of frequency of occurrence, they share one similar collocational pattern: that is hotels/restaurants/+ verbs (offering/providing/serving) +_+ services. An example is, "The Thai and European kitchen serves you many traditional Thai dishes..." (T10). Web designers use You as an indirect object of these following verbs: offer, provide, and serve to enhance the importance of the visitors and their need. Moreover, web designers use this pattern to promote hotel facilities and services.

The next collocational pattern of you that only occurs in the Thai dataset is_+verb (experiential verbs) +restaurant services and facilities. An example is " $\underline{Y o u}$ can enjoy exotic drinks, tasty snack and other delicious dishes from the pool bar" (T14). In this pattern, You performs an active role because it is used as the subject of the verb. The main purpose of this pattern is that the hotels intend to promote the restaurant services that the hotels provide. 


\subsection{Conclusion}

The register analysis covers three main types of prominent features: verbs, adjectives, and pronouns. Similarities in the prominent linguistic features between Thai and New Zealand hotel webpages are found in two moves - Introducing the hotel/facilities/services, and Establishing credentials. The similarities in the use of distinctive linguistic features across the two datasets were not satisfactorily clarified by the interviews with the hotel owners/managers, and web designers. This is because the hotel owners in New Zealand explained that they use their own intuition and feeling when writing hotel promotional texts. These similarities reflect the notion of conventions or regularities as suggested by Bhatia (1993); texts that are the same genre share some commonalities in terms of communicative purposes, textual features, and audience groups. On the other hand, minor differences in the use of the distinctive linguistic features were found in the other three moves - Describing the rooms, Calling for action and Describing offer. These minor variations across the two datasets seem to reflect the creativity of the expert in the discourse community and the dynamism of this hotel online advertising genre rather than cultural differences. 


\section{Chapter 7: VISUAL IMAGE ANALYSIS}

\subsection{Introduction}

The main communicative purpose of hotel webpages is to inform and promote the hotels, and this purpose is represented in the language and images used in the webpages. Following on from the discussion of the findings of textual analysis in chapters 5 and 6 , this chapter provides a visual analysis of six boutique hotel websites in Thailand and six in New Zealand.

To collect my datasets for my visual image analysis, I adopted content analysis to minimise the dataset size from 60 to six hotel websites. The reason that I had to minimise my dataset size was because each webpage contains several images, I could not include all 60 websites. I chose six hotels from each dataset (12 in total) based on the geographical proximity between the hotels for ease of transportation. That was because I aimed to interview the owners and/or staff members of these hotels after the textual and visual analysis.

Multimodality was used to analyse the hotels' visual communication with their target audience. This visual analysis also considers cross-cultural variation between the semiotic representation of Thai and New Zealand boutique hotel websites. The analysis draws on the framework of Kress \& van Leeuwen (2006). As previously discussed in chapter 4, section 4.3.3 Kress and van Leeuwen (2006)'s framework is extensive, relying on the generalisation of analysis of modern western visual materials, and has some elements that may be regarded as subjective. As a result, I have adopted this framework in particular the features that are practical to my dataset. Even though during my analysis, I discovered that some features may not be directly applicable to the context of my study, I continued using this framework because I wanted to see how the selected features would play out in my dataset. In regard to the issue of subjectivity, there are some features that I used in my analysis that can bear different interpretations; therefore, I used textual descriptions on the hotel webpages, interview data from the hotel owners, web designers, and web readers to support my analysis. My study focuses on the three metafunctions proposed by Kress and van Leeuwen (2006), which include the visual roles of images (representational meaning), the relationship between represented participants and viewers as well as the persuasive effect conveyed through represented participants (interactive meaning), and the layouts of the webpages (compositional meaning). Table 34 below describes the features that I adopted from the framework of Kress and van Leeuwen (2006). 
Table 34: The categories of features used in the visual analysis

\begin{tabular}{|c|c|c|}
\hline Metafunctions & Features on hotel webpages & What is realised \\
\hline Representational & $\begin{array}{l}\text { 1) What are depicted? } \\
\text { human/places/objects } \\
\text { 2) How the represented participants } \\
\text { are depicted? } \\
\quad \text { narrative/conceptual }\end{array}$ & $\begin{array}{l}\text { - human/non-human } \\
\text { - active/passive manner. }\end{array}$ \\
\hline Interactive & $\begin{array}{l}\text { 1) Imaginary relations between } \\
\text { represented participants and viewers } \\
\text { Gaze } \\
\text { demand/offer } \\
\text { Size of frame } \\
\text { close-up, medium, and long } \\
\text { shot } \\
\text { Perspective } \\
\text { frontal/oblique } \\
\text { vertical }\end{array}$ & $\begin{array}{l}\text { - eye contact } \\
\text { - distance between represented } \\
\text { participants and viewers } \\
\text { - involvement between viewers and } \\
\text { represented participants } \\
\text { - power relations between viewers and } \\
\text { represented participants }\end{array}$ \\
\hline Compositional & $\begin{array}{l}\text { 1) Template of webpages/ } \\
\text { position of visual elements on } \\
\text { webpages } \\
\text { left/right } \\
\text { top/bottom } \\
\text { centre/margin } \\
\text { triptych } \\
\text { 2) Salience }\end{array}$ & $\begin{array}{l}\text { - single/multiple template } \\
\text { - known/new information } \\
\text { - ideal/specific information } \\
\text { - critical information/subordinated } \\
\text { information } \\
\text { - use of color }\end{array}$ \\
\hline
\end{tabular}

The table 34 lists the features that I adopted from Kress and van Leeuwen (2006)'s visual grammar model that has been applied to analyse visual images in many contexts e.g., advertisements, tourist brochures, and hotel homepages. I discussed the details and examples of the framework in chapter 3, section 3.2.1. but I would like to provide a summary of the features that I used for my analysis here.

The first meaning is representational meaning. It refers to the characteristics of represented participants displayed in visual images. In my study, I examined how represented participants appeared and performed in their visual space and I analysed whether their roles were narrative or conceptual. If it is a narrative image, the represented participants are performing a particular action, which creates a vector. In contrast, a conceptual image does not have a vector. The roles of represented participants are static and passive. 
With interactive meaning, I observed how represented participants interacted with the viewers. In examining this meaning, I focused on three aspects, which include eye contact (gaze) and distance (size of frame) between the represented participants and the viewers. The last aspect within interactive meaning that I looked at is how represented participants are positioned in relation to the viewers (frontally, obliquely, or on a vertical angle).These three aspects reflect the relationship and power relations between images to the audience.

The final meaning is compositional meaning. I examined webpage layouts to see how the pages' visual elements were displayed. Kress and van Leeuwen's (2006) suggests that the positions of visual elements convey the importance of the promotional messages that the hotels aim to deliver to the viewers.

\subsubsection{Criteria for selection of hotel webpages}

This study focuses on how images are visually represented on the three main webpages Home, Suites, Facilities and Activities - of six boutique hotel websites in Thailand and six boutique hotel websites in New Zealand. However, hotels categorise and name their webpages differently according to what services and facilities they aim to promote. Generally, Home and Suites are the main pages that every hotel has, but the organization of facility related pages (e.g., Dining, Spa, Wedding, and Events) varies depending on the main focus of each hotel is. For example, the hotel in Figure 24 does not have a Facilities/Activities page. Their main focus is on their restaurant service and their spa, so it has separate Dining and Spa pages to attract potential guests. Even though the hotel does not have Facilities page, I still included the hotel in my dataset because their Dining and Spa pages represent their main facilities. In short, my analysis includes three main webpages: Home, Suites, and Facilities/Activities, and I also incorporated facilities-related pages (e.g., Dining, Spa, Wedding, and Events) for those hotels which had different webpage organisation. As previously discussed in section 4.2.1, since some hotels did not want to reveal their identities, I used alphabetised and numerical labels to refer to all of the hotels in my datasets (e.g., T1, T2, T3 [Thai hotels] and NZ1, NZ2, NZ3 [NZ hotels]). 


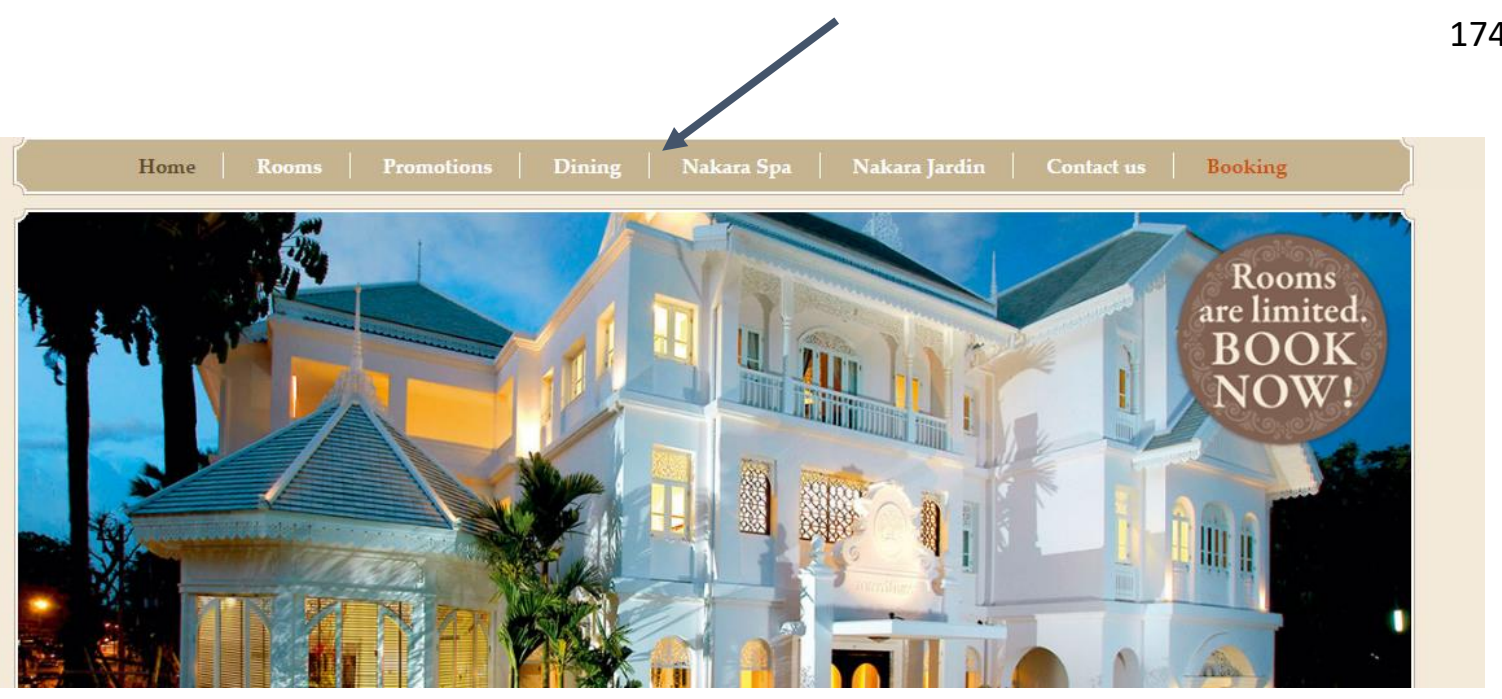

Figure 24: The homepage (T8)

Source: http://www.pingnakara.com/

\subsection{What images are presented on hotel webpages}

This section discusses the number of images depicted on the seven webpages (Home, Suites, Facilities/Activities, Dining, Spa, Wedding, and Events) in six hotel websites in the Thai and New Zealand datasets. The number of images presented on the webpages reflects the primary focus of the twelve hotels (six in Thailand and six in New Zealand) in advertising to viewers. The information about the number of pictures also enables me to compare the differences and similarities in the main focus of services and facilities between Thai hotels and New Zealand hotels. Table 35 shows the total number of images presented on seven categories of webpages of the six Thai hotels. The categories of webpages containing the largest number of images are Suites (49 images), Home (34 images), Dining (30 images), and Facilities/Activities (23 images). 
Table 35: The total number of images depicted on the Thai hotel webpages

\begin{tabular}{|l|c|c|c|c|c|c|c|c|}
\hline $\begin{array}{l}\text { Hotel in } \\
\text { Thailand }\end{array}$ & Home & Suites & $\begin{array}{c}\text { Facilities/ } \\
\text { Activities }\end{array}$ & Dining & Spa & Wedding & Events & Total \\
\hline T2 & 6 & 16 & 8 & 5 & & & & 35 \\
\hline T22 & 3 & 7 & 6 & 8 & & 4 & 3 & 31 \\
\hline T17 & 7 & 7 & & 7 & 7 & & & 28 \\
\hline T8 & 7 & 8 & & 5 & 5 & & & 25 \\
\hline T23 & 6 & 7 & 9 & & & & & 22 \\
\hline T21 & 5 & 4 & & 5 & & & & 14 \\
\hline Total & $\mathbf{3 4}$ & $\mathbf{4 9}$ & $\mathbf{2 3}$ & $\mathbf{3 0}$ & $\mathbf{1 2}$ & $\mathbf{4}$ & $\mathbf{3}$ & $\mathbf{1 5 5}$ \\
\hline
\end{tabular}

Table 36 presents the number of images on the seven categories of webpages of the six hotels in New Zealand. Webpages with the most images are Suite (41), Home (35), Facilities/Activities (25), Events (17), and Dining (13).

Table 36: The total number of images depicted on the New Zealand hotel webpages

\begin{tabular}{|l|c|c|c|c|c|c|c|c|}
\hline $\begin{array}{l}\text { Hotel in } \\
\text { New } \\
\text { Zealand }\end{array}$ & Home & Suites & $\begin{array}{l}\text { Facilities/ } \\
\text { Activities }\end{array}$ & Dining & Spa & Wedding & Events & Total \\
\hline NZ10 & 8 & 11 & 7 & 4 & 4 & & 4 & 38 \\
\hline NZ26 & 8 & 8 & 9 & & & & & 25 \\
\hline NZ9 & 8 & 9 & & & & & 9 & 26 \\
\hline NZ18 & 3 & 5 & 5 & 5 & & & & 18 \\
\hline NZ27 & 4 & 4 & & 4 & & & 4 & 16 \\
\hline NZ25 & 4 & 4 & 4 & & & & & 12 \\
\hline Total & $\mathbf{3 5}$ & $\mathbf{4 1}$ & $\mathbf{2 5}$ & $\mathbf{1 3}$ & $\mathbf{4}$ & & $\mathbf{1 7}$ & $\mathbf{1 3 2}$ \\
\hline
\end{tabular}

Overall, the boutique hotel webpages in Thailand contain more pictures than the New Zealand dataset. The Thai hotels use a total of 155 images across the seven webpages while the hotels in New Zealand use 132 images to advertise their hotels. Among the seven types of webpages 
(See Tables 35-36), the Home and Suites pages seem to be the most important pages in my dataset because these two pages occur on every hotel's website, those in Thailand and in New Zealand.

The Suites page contains the largest number of images across the two datasets - 49 images in the Thai dataset and 41 images in the New Zealand dataset. The second largest is the Home page, with 34 and 35 respectively. One reason why the Home page contains a large number of images and also occurs on every hotel website in my dataset is because it is the first page the readers see and presents the image of the hotel to the outside world. This is also congruent with Shepherd and Watters' (1999) and Nielsen and Tahir's (2002) suggestion that a homepage is used to promote the face that a company wants to present to the world. Nielsen and Tahir (2002) also indicate that homepages are influential because they are the most visited pages compared with other pages on the same websites. Another possible reason for the larger number of images on hotel homepages was given by the owner of $\mathrm{T} 2$ hotel. He stated that generally the hotel has less than 3 seconds to impress and hold the interest of the viewers. His comment is also echoed in Lindgaard, Fernandes, Dudek, and Brown's (2006) findings that effective web design and visual appeal create positive impressions for viewers within 500 milliseconds. As a result, it is vital that the Home page contains the most inviting images to attract and engage the viewers' attention in order to encourage them to click through to the other pages and make a reservation.

In addition to the importance of homepages, the Suite page in the Thai and New Zealand dataset also contains the largest number of images and functions to encourage the viewers to make a booking. The hotels aim to create income by presenting the information about the number, types, design and decoration of the rooms (theme), including the details of the facilities and amenities provided in each room. This suggestion is confirmed by the interview with the owners of the T8 and T24 hotels, who said that suites are the most important selling point for potential guests. This is the reason why owners put a lot of energy into the design and decoration of the accommodation. These two hotels use a theme to advertise their rooms, e.g., flowers and precious stones.

Apart from the similarities between the Thai and New Zealand hotels on the Suite page, some differences between these two countries' hotels are found on the Dining page. This is because the number of Dining page images on the websites of the Thai hotels is twice as many as those of the New Zealand hotels. The Dining page is very valuable to the boutique hotels in Thailand. According to the Tourism Authority of Thailand (TAT) (2014), Thai food has become worldrecognised and the TAT has launched many campaigns related to Thai cuisine with the aim of boosting tourism to Thailand in order to generate more income for the economy. Interview with the hotel owners suggested they are well aware of this fact, and they aim to make use of these strategic 
campaigns to increase their income by promoting their restaurant service with an emphasis on Thai cuisine on their websites. The Dining page contains the third largest number of photographs across the eight Thai pages and this page appears in five hotels out of six. The function of the Dining page in my Thai dataset is to promote traditional authentic Thai and international cuisine, including restaurants/bars atmosphere and service (e.g., T8 and T22 hotel in Table 37) to not only the inhouse guests but also guests who are visiting the restaurant only. In short, The Dining page in my Thai dataset is also one of the most important pages for the hotels to generate their income. The only hotel which does not make use of the Dining page is T23 hotel (Table 37) because the information about the hotel's restaurant-related services is incorporated into the Facilities and Activities pages. Additionally, the hotel restaurant's services may not be a primary focus of the hotel or a source of the main income because the hotel mainly offers simple Thai dishes and a casual style of dining, as can be seen in the following example: An all day dining place which offers a relaxed atmosphere with simple Thai dishes, freshest salads and sandwiches for lunch to an informal way of dining, tasty cocktails and wine. These two reasons explain the absence of a Dining page promoting the restaurant services in $\mathrm{T} 23$ hotel website.

In contrast, as can be seen in Table 36 in the New Zealand dataset, the Dining page has 13 images on six hotels' webpages compared to 30 images in the Thai dataset, which is less than half the size of the Thai dataset. There are a number of differences between the functions of the Dining page in the Thai dataset and that in the New Zealand dataset. These differences may be explained by the fact that there are some dissimilarities between the main focuses of the national tourism industries and the cultures in these two countries. Even though the hotels in both datasets position themselves as boutique establishments, they largely offer different kinds of services and facilities related to dining experiences. In the Thai hotels, most restaurants operate flexible hours. As a result, the guests are welcome to dine in or order room service as often as they desire, while the hotel restaurants in the New Zealand hotels tend to operate during specific periods of time, mainly for breakfast. The likely explanation is that the Thai boutique hotel market is somewhat larger than the New Zealand market, and the hotels are generally larger. Therefore, there are a larger number of hotel staff members running the Thai hotels, whereas the boutique hotels in the New Zealand dataset are usually run by the hotel owners and a couple of staff. Another important explanation is that some New Zealand hotels in my dataset provide kitchen facilities for guests who would like to cook, while the provision of kitchen facilities is entirely absent from the boutique hotels in the Thai dataset. Rather, the hotels in the Thai dataset generally encourage guests to try Thai traditional food provided by the hotel restaurants because restaurants are a major source of the hotels' income apart from accommodation. 
Regarding the Facilities/Activities page, the total number of photographs in the Thai boutique hotel websites is 23 images compared to 25 in the New Zealand dataset, which is comparable. Three Thai hotels out of six represent their facilities and services on the Facilities and Activities page. These hotels are $\mathrm{T} 2, \mathrm{~T} 22$, and $\mathrm{T} 23$. In contrast, the rest of the hotels do not have the Facilities and Activities page. For example, the T17 hotel (see Table 35) does not have a separate page advertising their facilities, although they use the Dining and Spa to advertise their spa and restaurant, which seem to be their major selling points. At the same time, in the New Zealand datasset, only two hotels (NZ9 and NZ27 see Table 36) do not have a separate page promoting their facilities. In addition to their accommodation, hotel NZ27 (see Table 36) mainly promotes their cuisine and function venue, which seems to be their main focus. For hotel NZ9 (see Table 36), the hotel arranges its facilities in a distinct manner. Hotel NZ9 (see Figure 25) has a Facility page, but it is located in a drop down menu under the Accommodation links. Consequently, this facility page is not included in my analysis. I focus on the pages that are shown on the navigation link menu of the hotel webpages as listed on Tables 35-36.

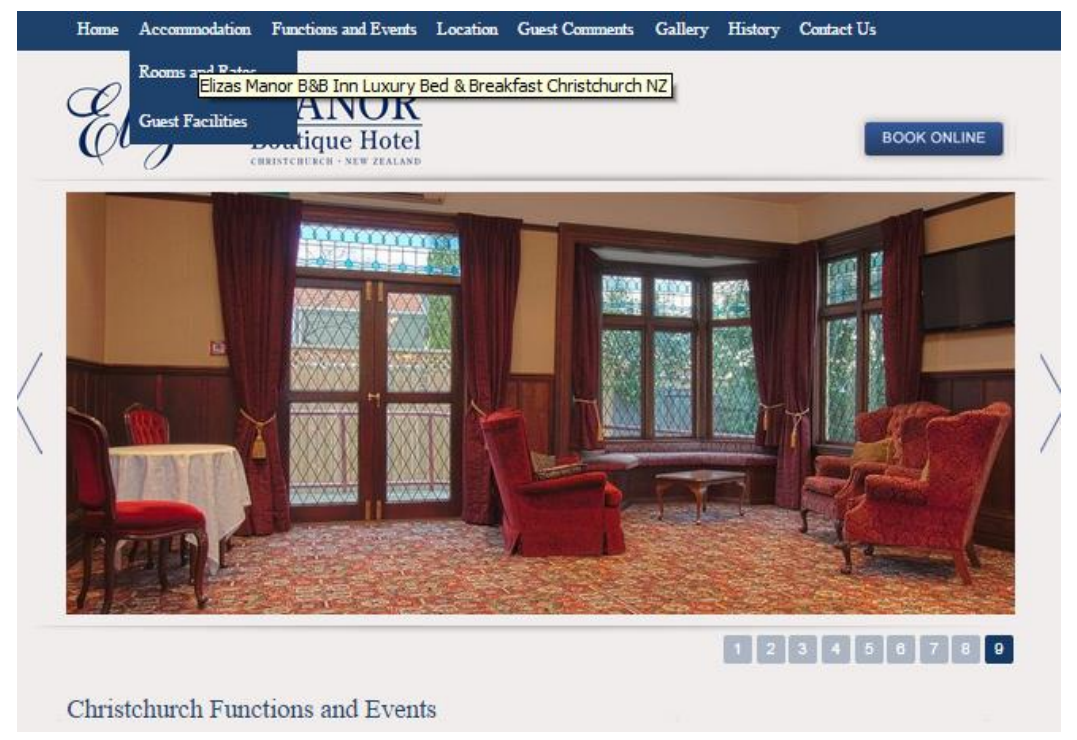

Figure 25: The accommodation page (NZ9)

Source: http://www.elizas.co.nz/functions-and-events

The Wedding page and Event page (see Table 35) on the Thai boutique hotel website contrastingly, have a small number compared to the Home, Suites, and Dining pages. The number of the images displayed on these two webpages is 4 and 3 respectively and only occurs on the website of hotel T22 (see Table 35), whose manager said the hotel is very proud of their outdoor venue for 
wedding parties. On the other hand, in the New Zealand dataset, two hotels (NZ9 and NZ27) out of six hotels promote their facilities and services on the Event page (see Table 36). The total number of images depicted on the Event page is 17, the third largest number in the New Zealand dataset and considerably larger than the number of images advertised on the Event pages of the hotels in the Thai dataset. This can be explained by the fact that the facilities and services-related to events and function venues are more significant in the New Zealand boutique hotel business than in the Thai boutique hotel business. This may be because the location of the New Zealand hotels is ideal, convenient, and suitable for having private events and meetings. For example: T27 Lodge is located on Waiheke Island, a 35-minute ferry ride from downtown Auckland (CBD), but a world away from the hectic metropolis. Our boutique Lodge is the ideal venue for business, corporate, and executive groups. In contrast, in Thailand, companies which would like to organise events and functions usually target big hotels in the city center rather than small boutique hotels. Regarding the Wedding page, none of the hotels in the New Zealand dataset exploited the Wedding page. Instead of advertising the hotel wedding facilities and services on a separate page (Wedding), these hotels (NZ10, NZ9, and NZ27) integrated their wedding promotional texts into the Event page. For example, Our dedicated team will work with you to design an unforgettable private or corporate function, conference, launch, anniversary or wedding in this 5-star luxury setting (NZ10).

Lastly, for the Spa page, the number of images in the Thai dataset is three times larger than that in the New Zealand dataset. There are twice as many images on the Thai Spa pages (12) compared to the New Zealand Spa pages (4). This difference may be caused by the fact that only NZ10 hotel has a Spa page, while two hotels in the Thai dataset have a Spa page. Also, in terms of the popularity of a spa service and guests' expectations of boutique hotel services in Thailand, the spa service seems to be the first option because Thai spas are very well- recognised for their expertise and reasonable prices. The above discussion shows how the number of images on each webpage in both datasets indicates the importance of types of facilities and services that the hotels aim to promote to their readers.

\subsection{Representational meaning}

The representational meaning is about what is depicted in an image. The represented participants (henceforth RPs) can be either people, places, or objects. The data reveals that there is a predominance of what researchers (Jewitt and Oyama 2001; Knox, 2007; Moya Guijarro \& Pinar Sanz, 2008; Oyama, 1999; Unworth, 2001) have termed conceptual images on the boutique hotel webpages in both the Thai and New Zealand datasets (see Table 37 below). According to Kress and van Leeuwen (2006, p. 79) conceptual structures refers to images which do not contain vectors, and 
RPs are described in a more static and passive manner. Conceptual images do not narrate a story but are described based on their physical spatial relations. On the other hand, narrative pictures are realised by a vector where participants are depicted as performing roles or doing something to or for each other. They visually present unfolding actions and events, process of change, and transitory spatial arrangements (ibid, p. 79).

Table 37: The total number of conceptual and narrative images depicted on the hotel webpages in the Thai and New Zealand datasets

\begin{tabular}{|l|c|c|l|c|c|}
\hline Conceptual images & $\begin{array}{l}\text { Thai } \\
\text { hotels }\end{array}$ & $\begin{array}{l}\text { NZ } \\
\text { hotels }\end{array}$ & Narrative images & $\begin{array}{l}\text { Thai } \\
\text { hotels }\end{array}$ & $\begin{array}{l}\text { NZ } \\
\text { hotels }\end{array}$ \\
\hline $\begin{array}{l}\text { Hotel's exterior/ } \\
\text { atmosphere }\end{array}$ & $\mathbf{4 1}$ & $\mathbf{4 0}$ & Spa & $\mathbf{2}$ & $\mathbf{2}$ \\
\hline Hotel suite & $\mathbf{3 9}$ & $\mathbf{2 6}$ & $\begin{array}{l}\text { Hotel's exterior/ } \\
\text { atmosphere }\end{array}$ & $\mathbf{3}$ & - \\
\hline Restaurant & $\mathbf{3 7}$ & $\mathbf{1 6}$ & Restaurant & 1 & - \\
\hline Hotel lobby/lounge & 10 & 10 & Hotel suite & 1 & - \\
\hline Food & 7 & 11 & Wedding & 1 & - \\
\hline bathroom & 4 & 3 & Hotel staff & 1 & - \\
\hline Spa & 3 & 2 & Artistic mood & 1 & - \\
\hline Artistic mood & 3 & 2 & Function room & - & $\mathbf{2}$ \\
\hline Wedding & 2 & - & Host & - & 1 \\
\hline Scenery & 1 & 14 & & $\mathbf{1 2}$ & $\mathbf{5}$ \\
\hline Animals & - & 2 & & & \\
\hline Indoor pool & - & 1 & & & \\
\hline Function room & - & 10 & & & \\
\hline & $\mathbf{1 4 7}$ & $\mathbf{1 3 7}$ & & & \\
\hline
\end{tabular}

From the perspective of the representational meaning (Kress \& van Leeuwen, 2006), hotels use a larger number of conceptual images than narrative pictures on their webpages. My analysis shows that the main purpose of using conceptual images in both Thailand and New Zealand boutique hotels is to promote a hotel's exterior and its atmosphere, hotel suites, and restaurants. Additionally, conceptual images are adopted to convey the idea of relaxation and coziness or elegance (see Figures 26-28) and luxury (see Figure 26). Some hotels represent both concepts in one hotel (the idea of relaxation and luxury). This will be explained in the next paragraph. In my dataset, 
narrative images are mainly used to depict images where there are human participants as main characters, such as in a hotel's exterior and atmosphere, spa, or function room. The following discusses some examples of conceptual and narrative images.

Figure 26-28 are examples of the hotels in my dataset which aim to convey the idea of relaxation and coziness through the depiction of conceptual images in their three main varieties of images (hotel's exterior and atmosphere, suites, and restaurant). Figure 26 depicts the exterior and architectural design of the main building of NZ25, New Zealand at twilight. The hotel has the light on both inside and outside the main building, which conveys a romantic and relaxing atmosphere. The wood and stone structure of the hotel looks harmonious with its surrounding environment. The hotel's intention with this image seems to be to show the hotel's exterior, design, and atmosphere as a whole. This image also seems to symbolise the relaxation and privacy of the hotel that the guests will have when they come to visit the hotel, as can be seen in the following example: Our luxury lodge offers true New Zealand beauty and utmost comfort in a relaxed, casual style so you feel instantly at home. This example enhances the combination of luxury and relaxation in one. The impression given by the photograph is than even though the hotel is luxurious, guests still feel relaxed and comfortable when staying there.

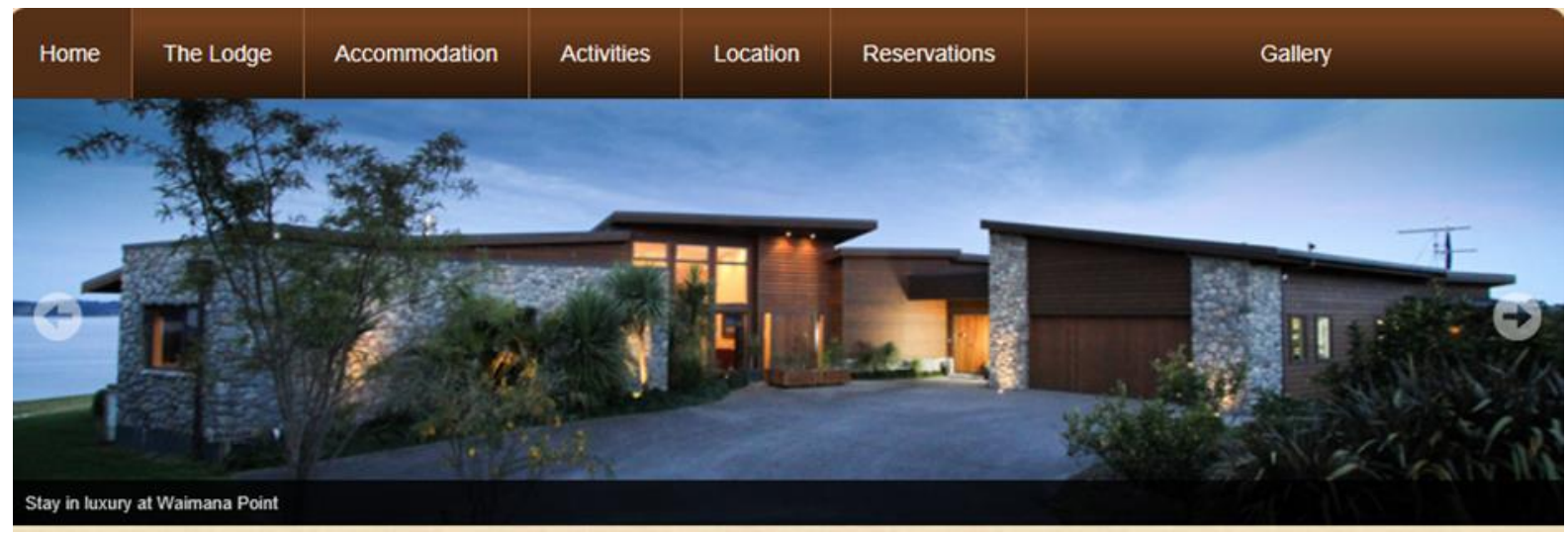

Figure 26: A hotel's exterior and atmosphere (NZ25)

Source: http://www.waimanapoint.co.nz/

Figure 27 is depicted on the accommodation page of NZ26, New Zealand. The names of all four rooms are the names of birds (kereku, tui, kotuku, and korimako), conveying the image of an eco or green hotel, as they suggest on their website. The hotel aims to enhance the idea of peacefulness, privacy, and relaxation at the hotel through the depiction of a room with a balcony overlooking a beautiful river and mountain views, along with the textual description as follows: Imagine a place so pristine, beautiful and peaceful that you will be immediately struck by the absolute silence and the sheer majesty of your surroundings. Also, the color of the room is a warm 
tone, which makes the room look cosy.The likely intention of the hotel through the adoption of this image is to encourage the viewers to come to enjoy and relax in beautiful scenery.

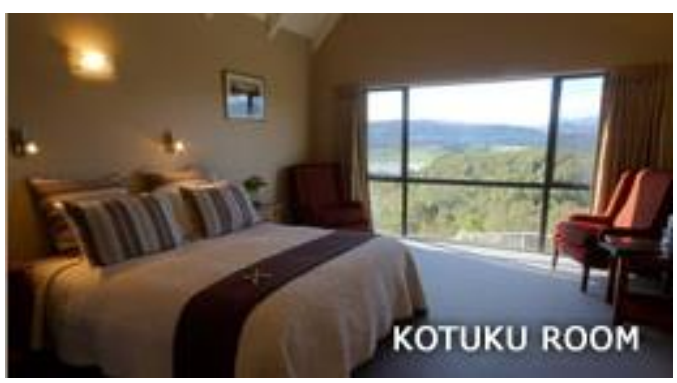

Figure 27: Suite (NZ26)

Source: $\underline{\text { http://www.rimulodge.co.nz/lodge.asp }}$

Figure 28 is a seaview restaurant depicted on T17's website. The image is captured from inside the hotel, as if the viewers were indulging in the peaceful scenery and calm sea at sunset while sitting inside the restaurant. This image creates a relaxing mood, and encourages viewers to come and enjoy the food and relaxing atmosphere.

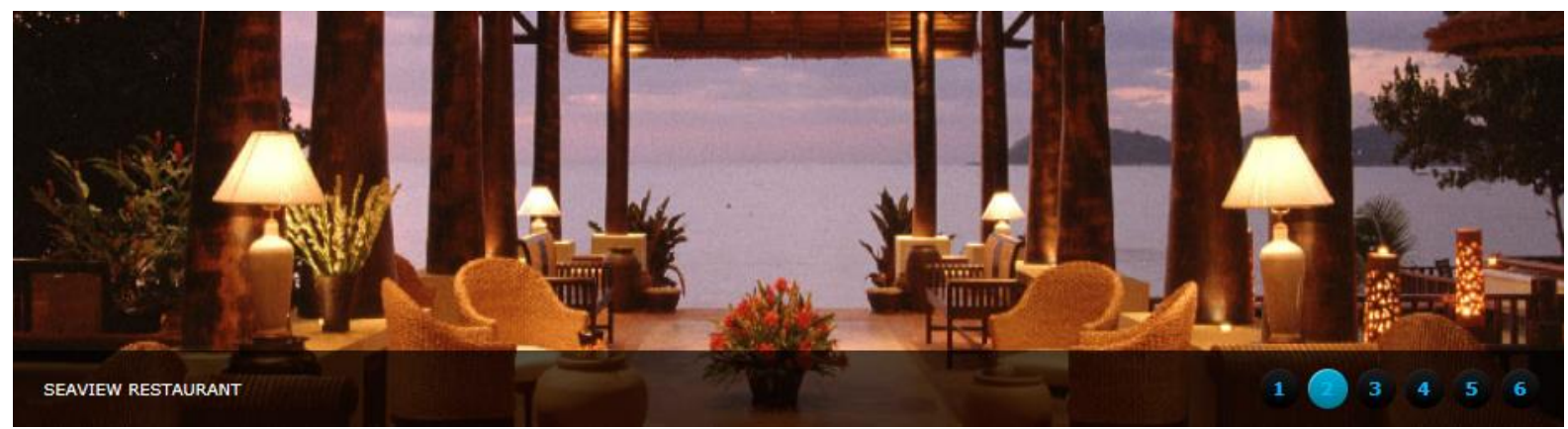

Figure 28: Restaurant (T17)

Source: http://www.levimarncottage.com/restaurant-\&-spa

Another function of conceptual images is to represent the notion of luxury and elegance. Figure 29 features NZ9, whose owners aim to emphasise the elegance and classic nature of their 1861 historic building. The elegance and luxury of this hotel is reflected in the design of beautiful antique ivory timber that the owners try to keep in its original condition. According to Chapman (2010) the ivory color evokes a sense of history, which corresponds to the main intention of the owners as they attempt to preserve this elegant architecture in its original state. 


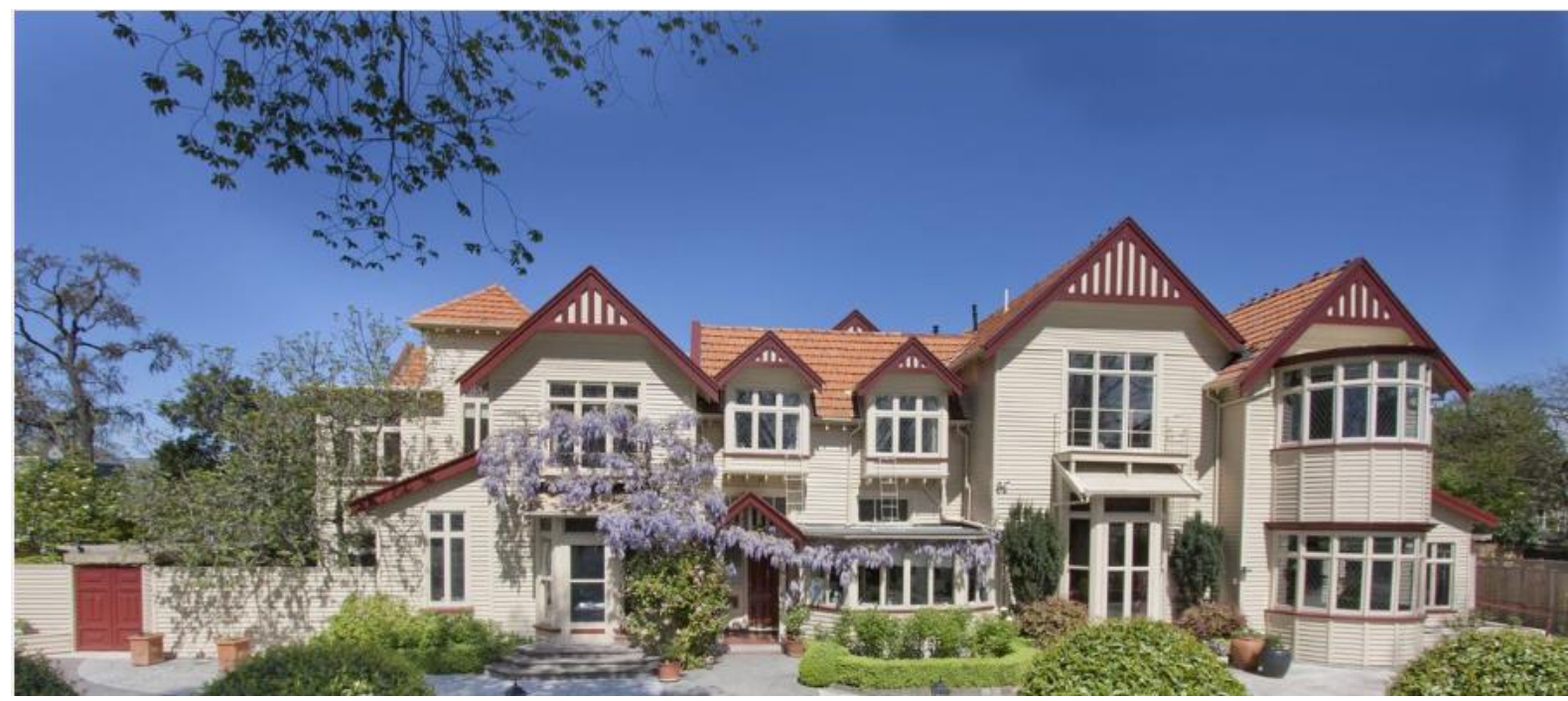

Figure 29: The exterior NZ9

Source: http://www.elizas.co.nz/

Figure 30 depicts one of the 19 suites on T8's webpages. All the 19 Lanna Colonial suites are uniquely decorated with different themes following the wall painting of flowers above the beds. The names of the rooms have been given the names of flowers. This conceptual image shows the details of room design and the teakwood furniture, which amplifies the luxury and elegance of the hotel. Also, the light reflecting from the bedside lamps and the bathroom make the room look cosy. The cream color and the design on the website are congruent with the main intention of the owner in establishing this as an elegant, luxury hotel.

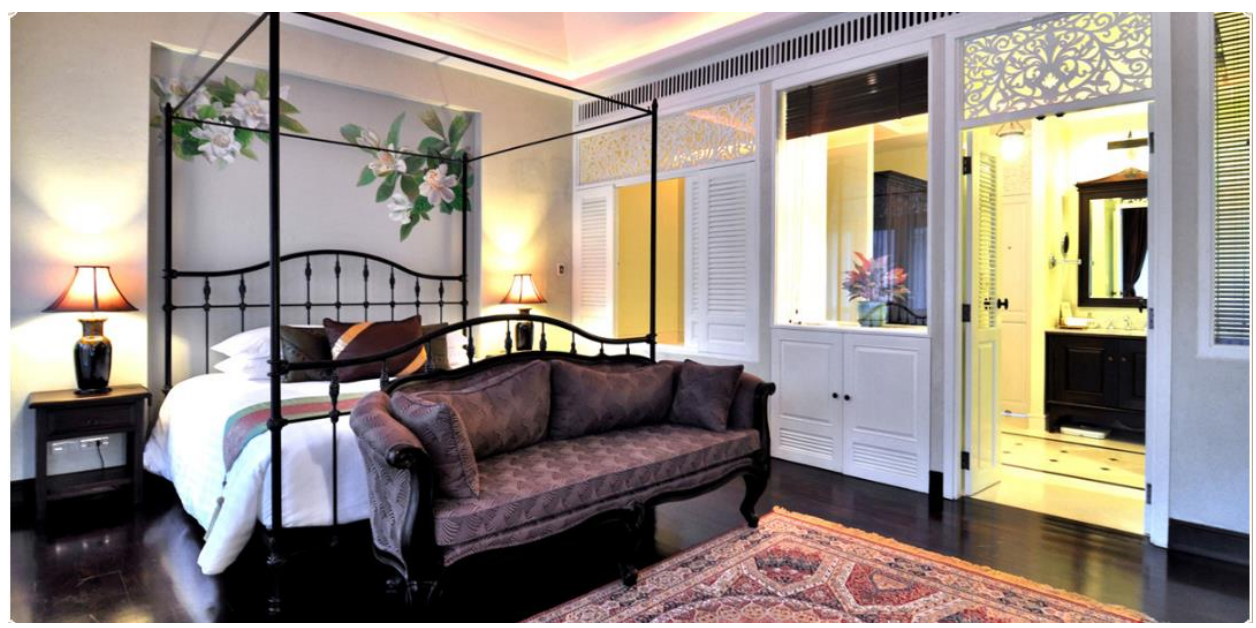

Figure 30: Suite (T8)

Source: http://www.pingnakara.com/room 
Figure 31 shows a dining room advertised on the Dining page of hotel T22. The craft wood decorations of the restaurant and table settings with a white table cloth and candle convey the idea of elegance and luxury and match the main concept of the hotel, which is a luxury boutique hotel.

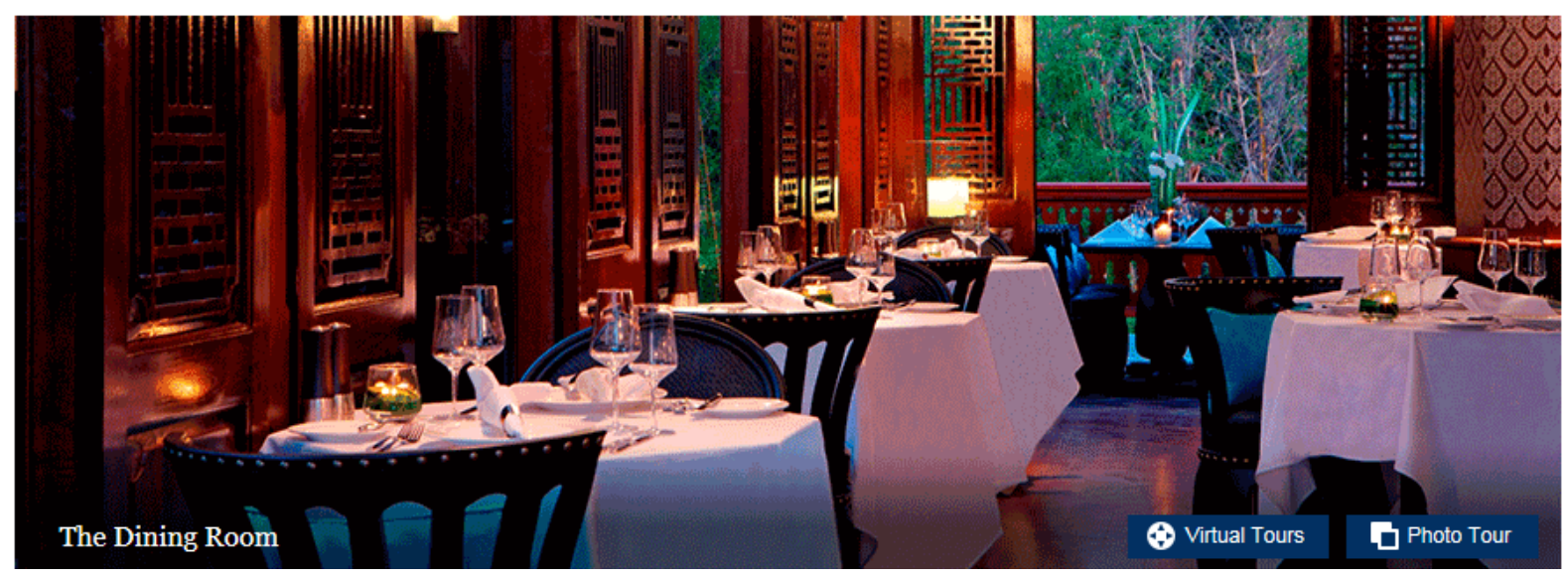

Figure 31: Dining room (T22)

Source: http://www.snhcollection.com/137pillarshouse/dining/

The narrative images in my dataset are mainly used to depict the images in which human participants are the main characters for example, in the hotel's exterior and atmosphere, spa, and function room. Jeong and Choi (2004) suggest that representation of human participants is important because it enhances the atmosphere of the hotels and allows readers to visualise how RPs would enjoy their experience at the hotels. Similarly, one of the Thai web designers explained that adding photographs with people makes the images look more real and life-like. However, a depiction of human RPs is expensive and not common because the hotels have to spend more money on their production budget to hire models.

Additionally, web designers explained that techniques used to select images depend on the experiences and personal preferences of web designers. Interestingly, there are no exact guidelines or rules for how to choose images. Generally, both web designers suggested that the photos depicted on hotel webpages should enhance and strengthen the characteristics of the hotels. The interviews with the manager of T22 affirmed the previous comments made by the two web designers. He said that "the choice of images is important because good images should tell a story about the hotel or portray the characteristics of the hotel". Another confirmation from the owner of T21 is that the purpose of his hotel website is to sell atmosphere and create mood; it does not just sell suites and facilities. As a result, there are no images depicting human participants in his website. Most images present suites and in one case a restaurant overlooking a river at night time (see Figure 
32). This technique is quite different from the conventional practice that some hotels employ when promoting restaurants. Often, photos that are selected to advertise hotel restaurants depict food or drinks in an attempt to create a mouth-watering effect as can be seen in Figure 33.

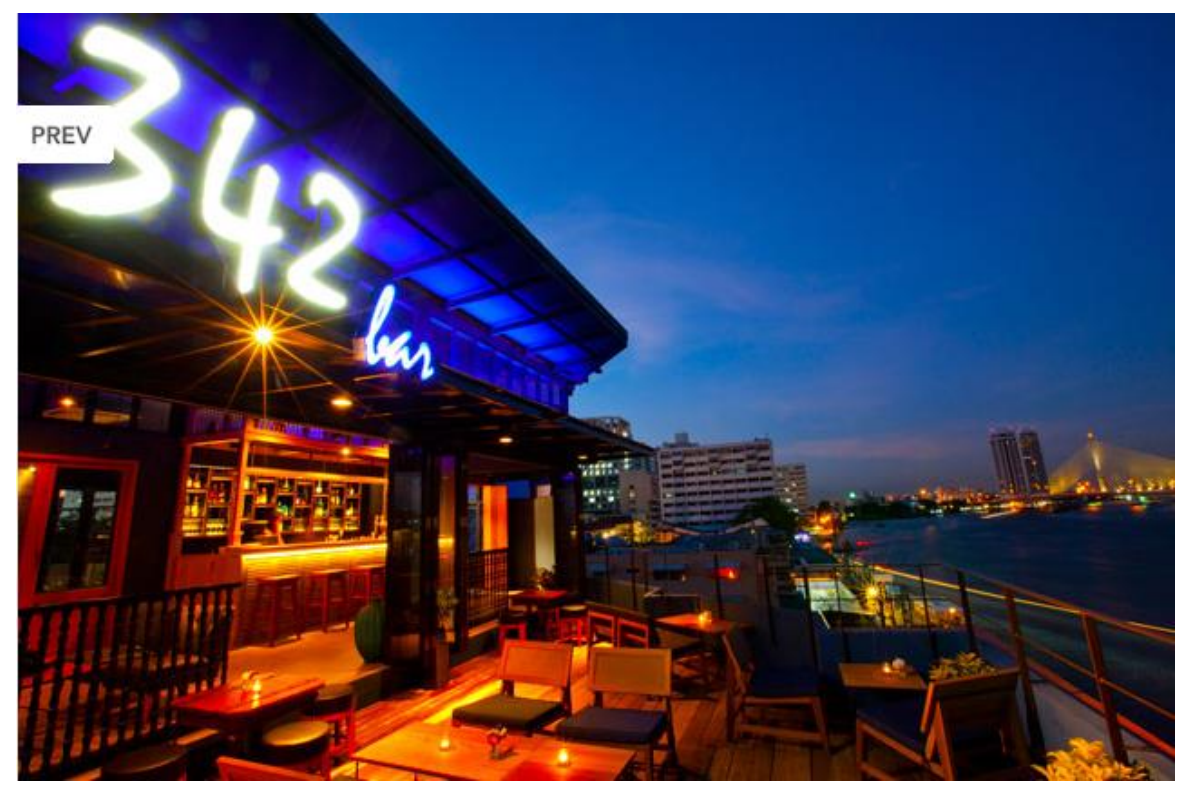

Figure 32: Riverside bar (T21)

Source: http://www.baanwanglang.com/photo_gallery.php 


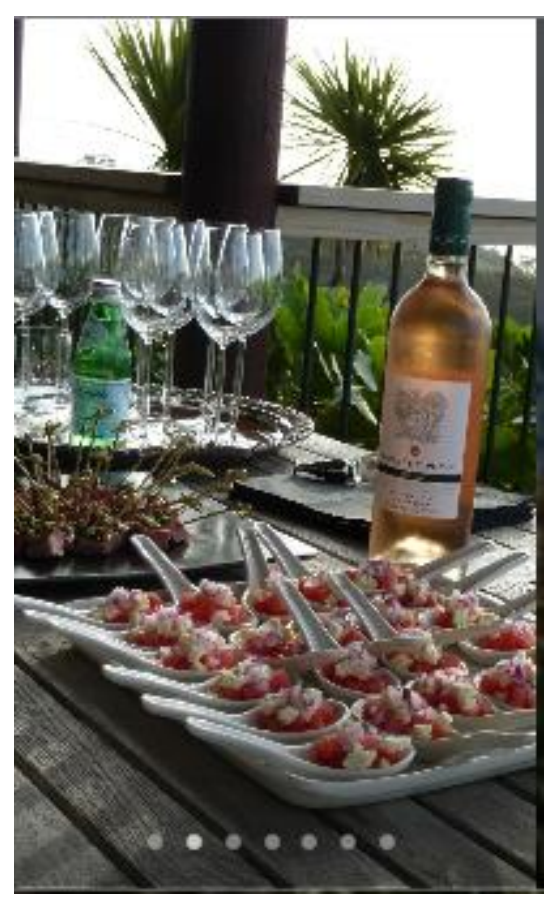

Figure 33: Restaurant service (T27)

Source: http://www.tewhaulodge.co.nz/cgi-sys/defaultwebpage.cgi

As the previous section focuses on the main objectives of using conceptual images on hotel webpages, this section discusses how narrative images are used to convey promotional messages. According to Kress and van Leeuwen (2006, p. 42), narrative images are realised by the presence of a vector that connects participants. The vectors represent the action of doing or a happening kind of relationship, such as when the hotel staff prepare baths for guests (see Figure 34). Figure 34 also aims to enhance the images of quality of service of the hotel as a luxury boutique hotel by depicting the hotel staff member paying attention to every detail in order to impress guests. However, it is not common in my dataset for the hotels to use narrative images to represent suites. Most hotels tend to use conceptual images, where there are no human characters to advertise the design and facilities of the rooms. Interviews with web designers also confirms this practice. Web designer 1 explained that narrative images are used in certain situations but are rarely used to promote suites. That is because narrative images, capturing human characters tend to distract readers' attention from the details of facilities and design of the suites. Figure 34 depicts a narrative image in which the actor or the doer of the action is the hotel staff member from whom the vector emanates. According to Jewitt and Oyama (2001), goals refer to the RPs at whom the vector is directed. Here, the vector is formed by the downward diagonal vector of the hotel staff member's arm and her eye line. The vector moves the viewers' focus from the hand toward the roses and then the bath. The roses in her 
hand and the bath are represented as a goal. Since Figure 34 involves both an actor and a goal, this narrative image is called a transactional structure (ibid, p. 143).

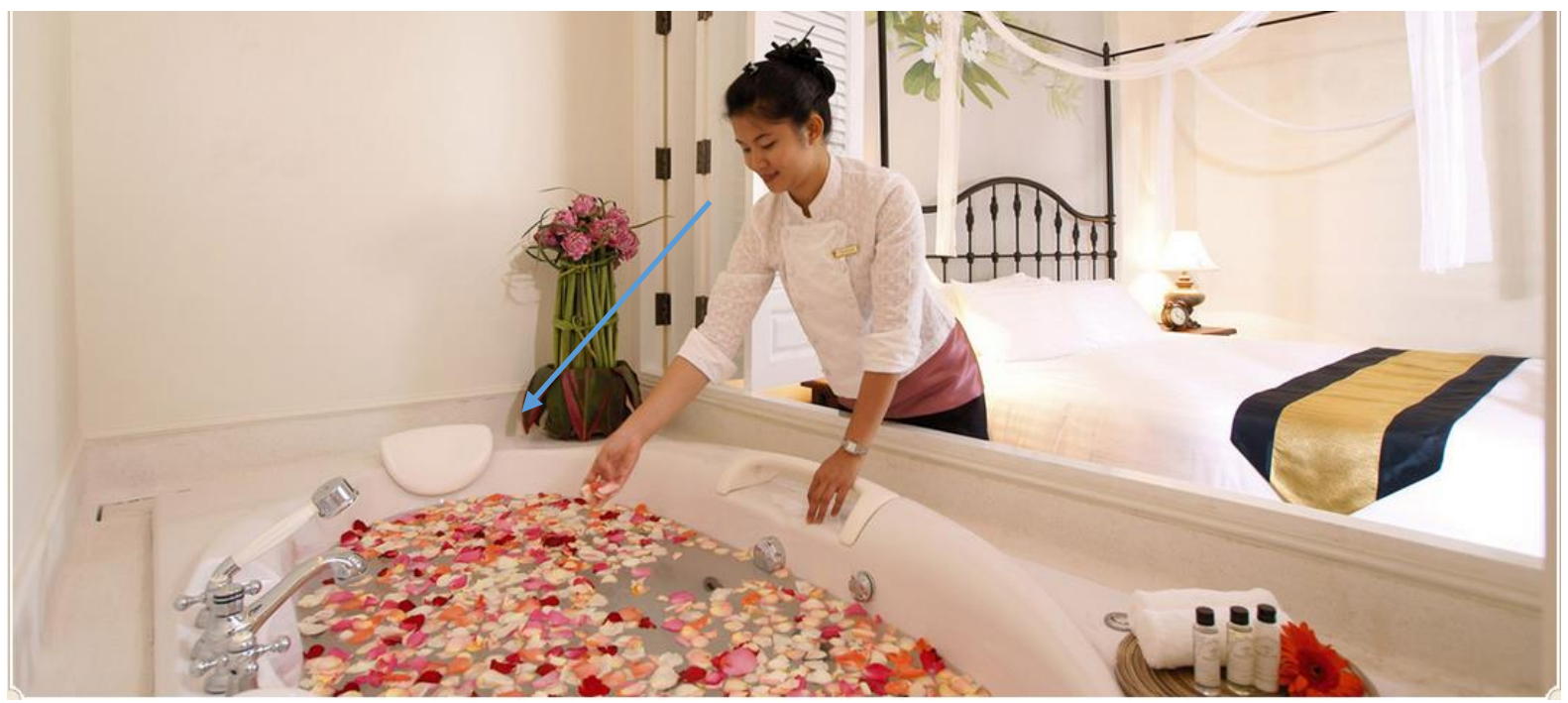

Figure 34: Hotel staff prepares a bath (T8)

Source: http://www.pingnakara.com/rooms

Figure 34 is an example of narrative image which shows action. The vector of action emanates from the direction of the massage therapist's arm and her eye line, which corresponds to Unsworth's (2001) suggestion that vectors are usually created by body structures or instruments in action (p. 73). The massage therapist is looking at the guest, who is enjoying having a massage. The vector lines are created from her gaze looking at the guest and by her arms massaging the guest's back. The massage therapist is represented as an actor and the guest is described as a goal of the image. 


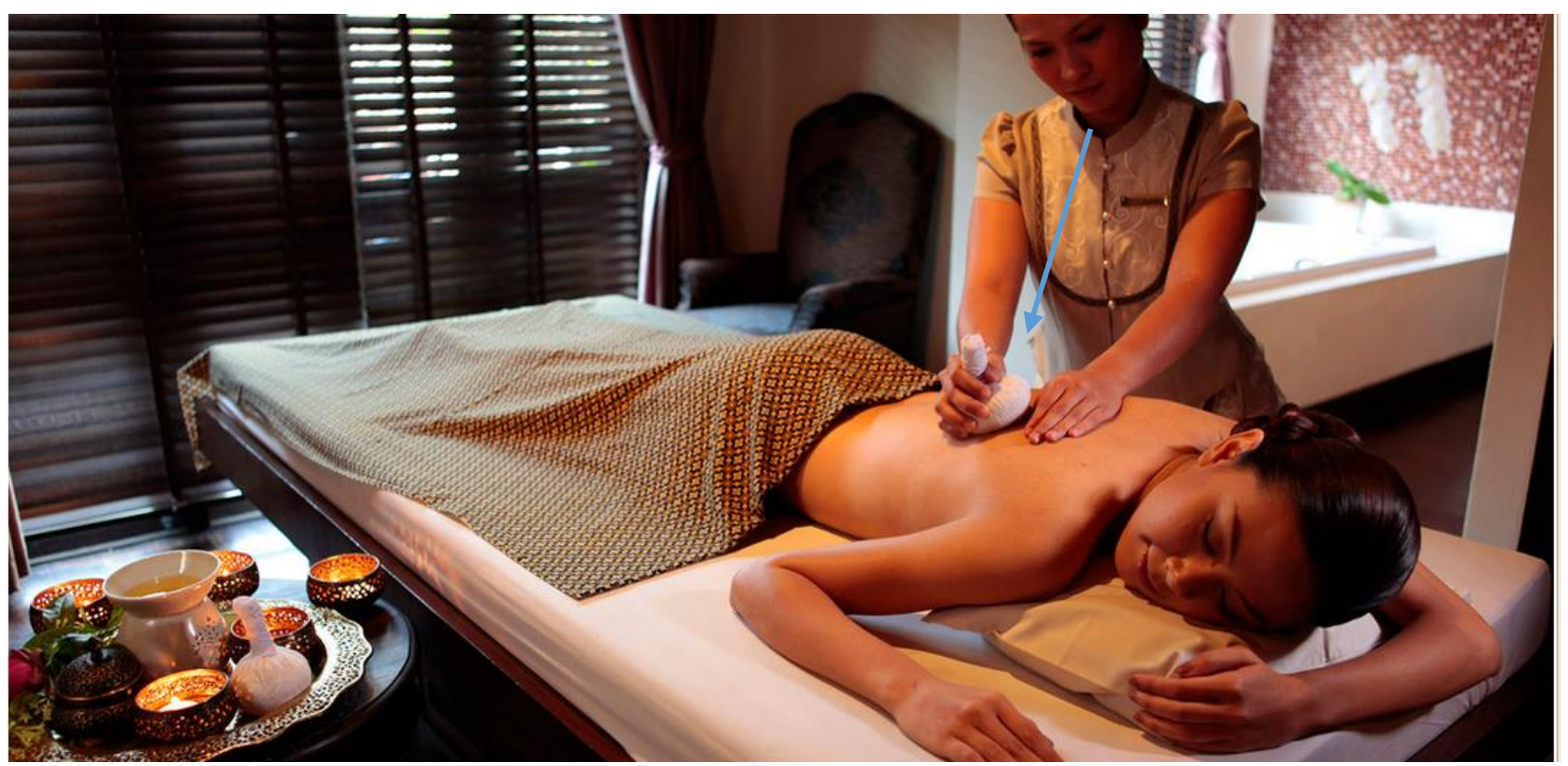

Figure 35: Spa (T8)

Source: http://www.pingnakara.com/spa

Figure 35 shows a narrative image representing unfolding actions at a function venue held at NZ9. The RPs are the guests who participate in the function organised by the hotel. To further analyse this narrative image, following the framework of Kress \& van Leeuwen (2006), Jewitt \& Oyama (2001) and Unsworth (2001), the eye line or the direction of the gaze of the RPs A and B (see Figure 36) constructs a reaction process in which the represented participants (the guests) are depicted as looking at the camera or the photographer who took this image. Jewitt and Oyama (2001) and Unsworth's (2001) note that the eye line or the direction of the gaze of the actor constructs a reaction instead of an action. Then the RPs are described as reactors and the object (the camera) or the person (the photographer) that the reactors are looking at are referred to as a phenomenon. At the same time, the RPs C, D, and E (See Figure 36) perform a different action. The participants are looking at something but there is no apparent participant to whom the action is directed to so there is no phenomenon (Unsworth, 2001). In this case, this image is called nontransactional (ibid, p. 73) as the RPs are presented as looking at something/someone but we do not know what/whom they are looking at. 


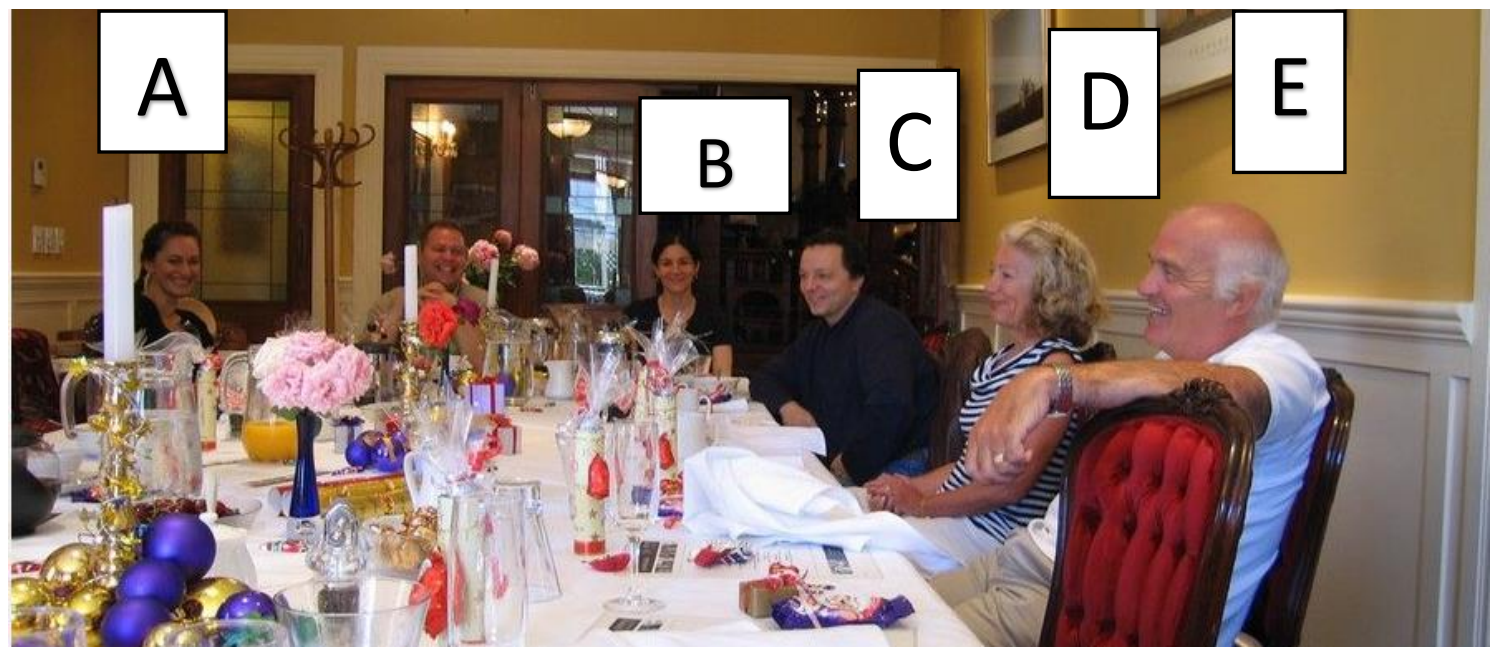

Figure 36: A Function room (NZ9)

Source: http://www.elizas.co.nz/functions-and-events

In some photographs, the distinction between conceptual and narrative images is unclear when there are no human characters. For example, Figure 37 can be either considered as a narrative image or conceptual image (Kress \& van Leeuwen, 2006, p. 46). By looking at the vectors on the wall, as shown by the arrows, this image can be seen as a narrative.The narration is realised through the vectors drawing the readers'attention and creating movement of the readers'eyes. The vectors lead the viewers to look at the interior of the villa and the decoration of the bed. The walls of the suites are the actors (ibid 59), which converge on the back of the villa and lead the readers'eyes to the back of the villa. At the same time, Figure 37 can also be described as conceptual where the hotel mainly aims to promote the overall atmosphere and highlights the beautiful wooden design and decoration that the hotel owner is proud of. The above section pays attention to the roles and functions of conceptual and narrative images used in my analysis. The following section focuses on the interactive meaning, which involves how RPs create a relationship and power with viewers through the use of eye contact, distance, and position in an image.

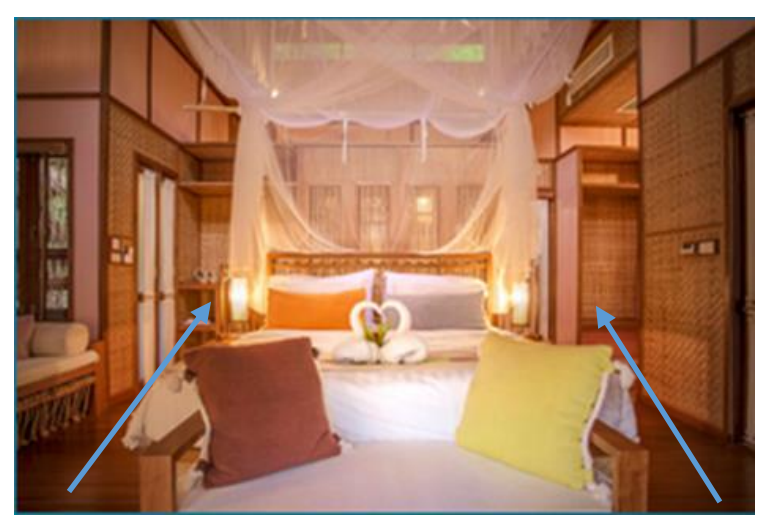

\section{Figure 37: Suite (T2)}

Source: http://www.thefloathouseriverkwai.com/kanchanaburi_hotels/rooms/en 


\subsection{Interactive meaning}

As discussed in section 3.2.1, the interactive meaning is concerned with the pseudo-social interactive relationship (Unsworth \& Wheeler, 2002) or imaginary relations (Jewitt \& Oyama, 2001, p. 145) between the RPs and the viewers. In my dataset, the RPs who play an important role in the interaction between the visuals and the viewers are as shown in Figure 38.

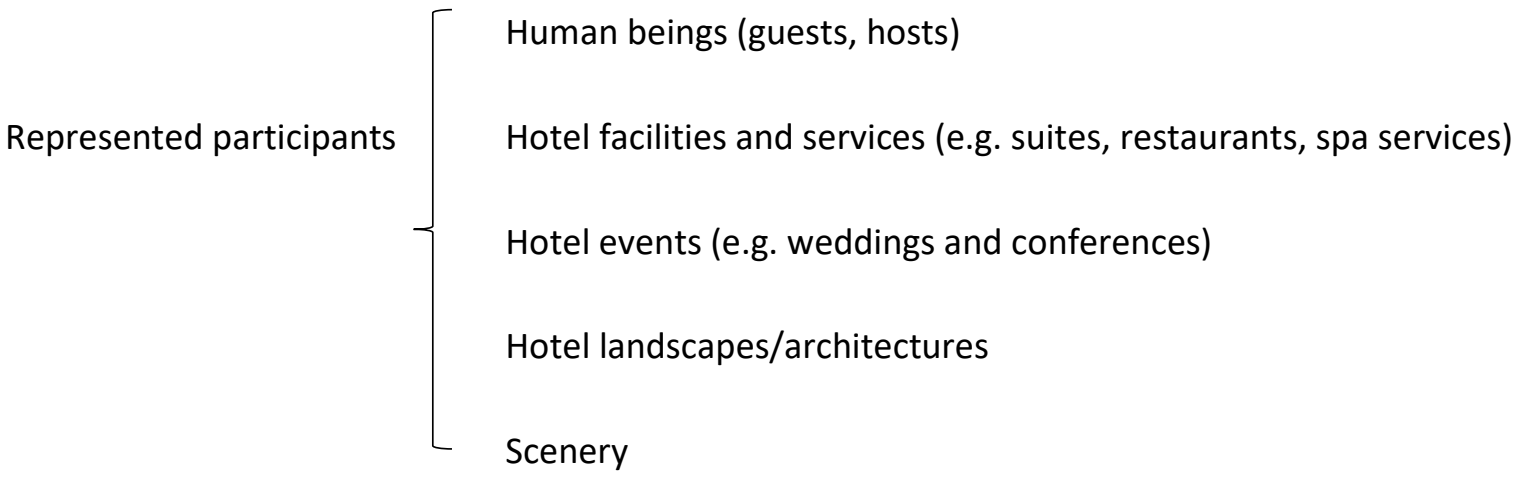

Figure 38: An example of the represented participants found in my datasets

Interpersonally, this interactive meaning is realised by the adoption of gaze, size of frame, and perspective, (Kress \& van Leeuwen, 2006; Francesconi, 2011). With written text, the relationship between the hotels and the readers is clearer and more straightforward than that of visual elements to investigate because there are certain linguistic devices available (e.g., pronouns and imperatives). In contrast, it is more complicated to examine how a relationship is constructed through a presentation of visual elements. In order to explore how web designers engage and maintain their interaction with their audience, apart from focusing on the use of gaze, size of frame, and perspective, the contexts where the images are presented, and the intention of the hotel owners and web designers need to be considered. My study explores how hotel owners and web designers establish a relationship with readers and potential guests through visual images.

Gaze refers to whether human represented participants have eye contact with viewers in order to establish an imaginary relation with them and invite them to engage interpersonally with the RPs. In this manner, the type of gaze described is referred to as a demand (Jewitt \& Oyama, 2001, p. 145; Unsworth, 2001, p. 95). In contrast, when the RPs do not have eye contact with the viewers, there is no pseudo-social interactive relationship (Unsworth \& Wheeler, 2002) between the viewer and the RPs, as a result, this image is considered as an offer (Kress \& van Leeuwen, 2006, p. 119). Even though, theoretically, contact or gaze is mainly delivered by the eye contact of human represented participants, this function can be also similarly realised by non-human RPs (Oyama, 
1999, p. 116). Oyama (1999, p. 116) explains that by considering the degree of salience of images and the degree of intensity of the image act, images which contain non-human participants can achieve similar functions to the interactive meanings of contact. This is contrary to Kress and van Leeuwen's framework where they suggest that gaze is mainly achieved through human, anthropomorphised objects or animals' eyelines. Applying gaze to non-human RPs in the analysis of boutique hotel websites appears very subjective because most images represent the hotel's architecture, suites, and restaurants; there is no parallel and comparable indication of eye contact similar to human's eye contact. In addition to the eye contact, which constructs relationship between the RPs and the viewers, facial expression also highlights interpersonal meaning (Kress \& van Leeuwen, 2006, p. 118; Unsworth, 2010, p. 95; Machin, 2007, p. 111; Oyama, 1999, p. 188). In regard to the analysis of gaze in my dataset, I therefore pay attention only to human represented participants as can be seen in the next section.

\subsubsection{Gaze}

Based on interactive structure proposed by Kress and van Leeuwen (2006), the number of images that adopt human RPs as a main character is only a total of 13 images out of 155 in the Thai dataset and 13 out of 132 in the New Zealand hotels. Interview with web designer 1 explained the reasons behind the infrequent use of human RPs that the hotels have to invest larger amount of money to hire models to display on the hotel websites. Occasionally, hotels hire guests who are staying at the hotel to be a model in exchange for free accommodation and food. In addition, human RPs are mainly used in certain images, such as in weddings, events, restaurants and spas. As a result, there are very few images that adopt human gaze to represent interactive meaning in my dataset. In addition, among the images that use human gaze, offer images are more frequent than demand images the boutique hotels in both the the Thai and New Zealand datasets (see Table 38).

Table 38: The number of gaze images in the Thai and New Zealand dataset

\begin{tabular}{|l|l|l|}
\hline $\begin{array}{c}\text { Types of } \\
\text { gaze }\end{array}$ & \multicolumn{1}{|c|}{$\begin{array}{c}\text { Total of number of images in Thai } \\
\text { dataset }\end{array}$} & $\begin{array}{c}\text { Total of number of images in New- } \\
\text { Zealand dataset }\end{array}$ \\
\hline Offer & 12 & 12 \\
\hline Demand & 1 & 1 \\
\hline
\end{tabular}

Table 38 demonstrates that there is a similar number of offer (12 images) and demand images ( 1 image) depicted on the boutique hotel websites in Thailand and New Zealand. The numbers show that the boutique hotel webpages usually depict offer images where human RPs 
make an offer with an indirect eye contact with the viewers. Kress and van Leeuwen (2006) suggest that this depicts RPs as if they were specimens in a display case (Kress and van Leeuwen, 2006, p. 124). However, Kress and van Leeuwen's (2006) theory does not correspond to the context of boutique hotel web advertising. My analysis revealed that offer images are used to show that the human RPs who are depicted as hotel guests are enjoying their holiday at the hotels and the viewers can also have similar experience when staying at their hotels. For example, Figure 39 and Figure 40 show examples of the offer images in which the human RPs make an indirect gaze to the viewers. Both images depict the hotel restaurants where the human RPs are sitting. Figure 39 depicts RPs as a couple guests having a drink in the hotel restaurant. There is no direct gaze towards the viewers to demand the viewers to come into the RPs' world. From their facial expression, the hotel aims to tell the readers that the RPs are enjoying their time. It seems that the hotel uses this image as an inviting message to convince the viewers that they can similarly have a delightful experience like this couple when they are using the hotel restaurant service.

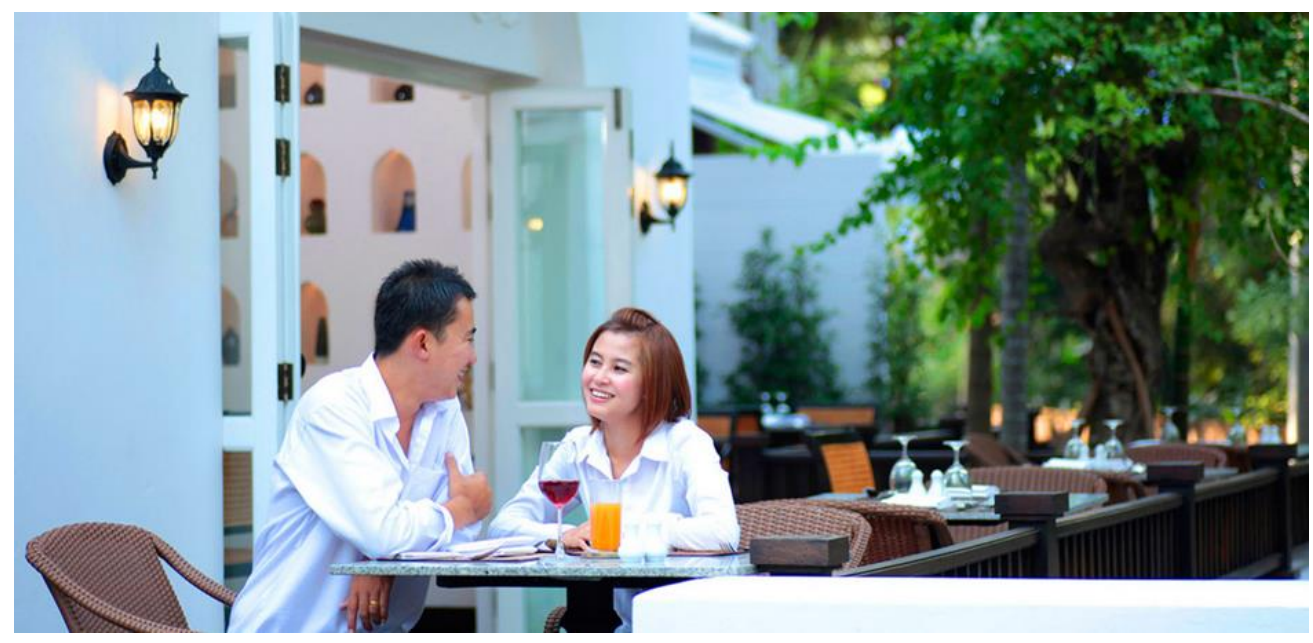

Figure 39: Offer image (T8)

Source: http://www.pingnakara.com/dining

As the visual elements in Figure 40 are not clear and are depicted with a long shot, it is difficult to describe what the main characters are doing. It is likely to be three groups of guests at the tables in the hotel restaurant. From the semi-formal clothing that the guests are wearing and the location of the hotel that is situated in the city, the guests could possibly be business people who are having a business talk with their business partners or clients. None of the represented participants engage in a direct gaze towards the viewers. It seems there is no possibility of interaction between the viewer and the characters being represented, since the viewers only see their backs and their body from the side. Viewers are not personally invited to join their conversation. This image seems to enhance the viewers' identification with the restaurant atmosphere and design rather than the 
interaction between the characters and the viewers as the camera focuses on the fireplace and the restaurant setting as a whole. The interaction between the human characters and the viewers is unlikely to be the main focus of the hotel. The human participants seem to be small ingredients to enhance the liveliness of the restaurant environment. The main focus of the image seems to highlight the cosy ambience and the decoration of the hotel restaurant.

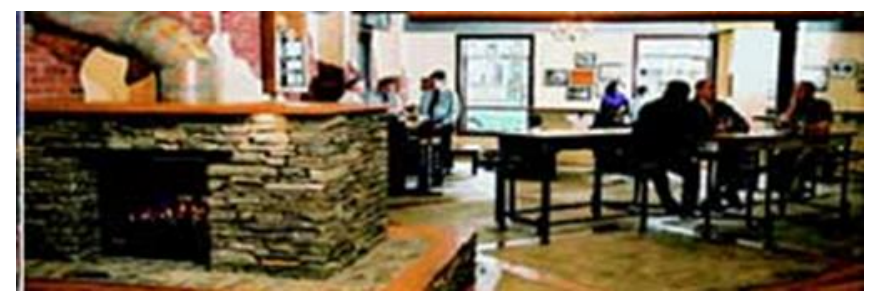

Figure 40: Offer image (NZ18)

Note: The hotel has now changed their website. This photo was used on the old webpage

There are only two images that are realised as demand images (Figures 41-42) in the boutique hotel webpages in Thailand and in New Zealand. Figure 41 depicts human RPs who are the hosts standing at the hotel reception counter. Figure 42 represents a hotel staff member who is holding a glass of water in her hand. In regard to gaze, the human RPs in these two figures make a demand wth a direct gaze at viewers. The hotels aim to establish some kind of imaginary relations with viewers (Kress \& van Leeuwen, 2006, p. 250). Following the framework of the researchers (Jewitt \& Unsworth, 2010, p. 95; Kress \& van Leeuwen, 2006, p. 118; Machin, 2007, p. 111; Oyama, 1999, p. 188), the interpretations of visual images where human beings are depicted as main characters are also influenced by gestures and facial expressions. In this case, the hotel chooses Figure 41 and 42 where the RPs are smiling and looking at the viewers in order to reflect their friendly and warm welcoming. The interview with the host of NZ9 also supports the interpretation that they use this demand image to encourage and invite the potential guests to come to visit the hotel. 


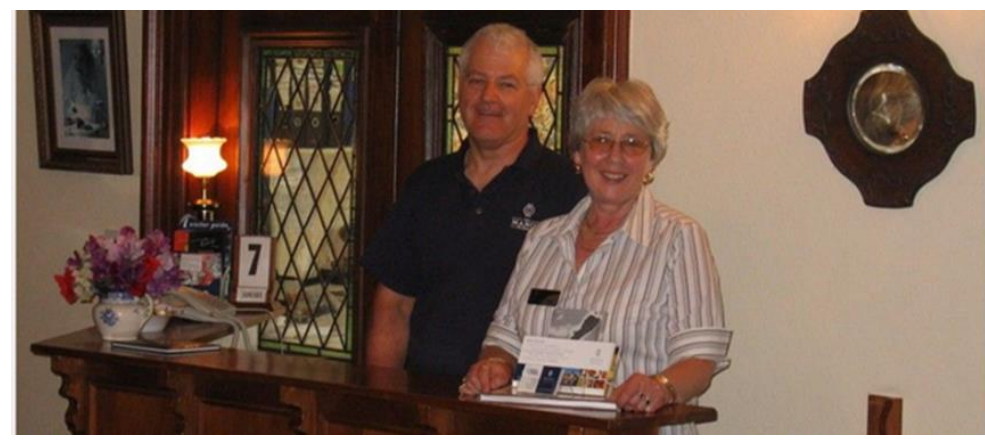

Figure 41: Demand image (NZ9)

Source: http://www.elizas.co.nz/

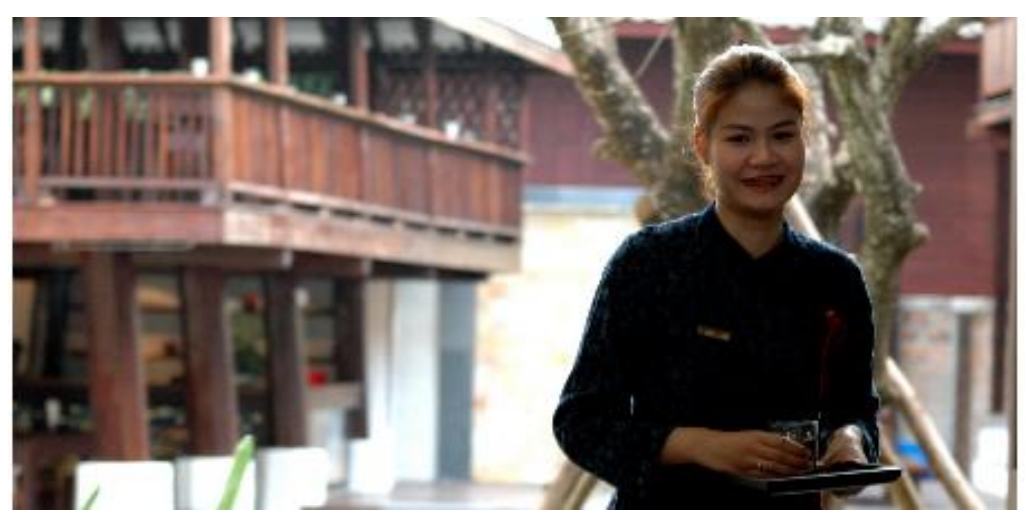

Figure 42: Demand image (T23)

Source: http://www.banthaivillage.com/facilities.php

There are a few cases in the analysis where some images contain both offer and demand in one image. Sometimes it is not clear if the human's gaze is making an offer or a demand on the viewers. For example, Figure 43 is from a homepage providing a general description of the hotel to web viewers. It features human RPs who appear as guests and hotel staff. One group depicts guests who are wearing dark clothing and enjoying their conversation with friends or family on the veranda of the second floor of the hotel. In regard to Interactive meaning, they do not have eye contact with the viewers and they are seen from the side at an oblique angle. As a result, the image is considered as an offer according to Kress and van Leeuwen (2006). It implies that there is no interpersonal relationship constructed between the images and the viewers. Viewers are not personally invited to join this conversation. The hotels aim to show the atmosphere of the hotel as a whole where the guests are enjoying their time while staying there. Another possible intention of the hotel is to convey the message that even though the hotel is small, the guests still have their space and privacy to enjoy their time with friends and family. Owing to the poor quality of images, it is not clear if the human RPs are making an offer or if they demand engagement with the viewers.

Figure 43 depicts another group of RPs who are hotel staff members. They wear a dark uniform and are depicted with a frontal angle as they are looking at the web readers. As the image is 
small and depicted in long shot, it is not clear whom the vector line is addressed to. It might be the camera or viewers. In addition, since the interpretations of visual images are also influenced by gestures and facial expressions (Jewitt \& Oyama, 2001; Unsworth, 2010), by looking at the hotel staff members, it is difficult to confirm that they are welcoming their guests from their facial expression Consequently, it is not explicitly clear whether this image is demand or offer. However, interview with the hotel owner confirms that the hotel aims to promote the hotel's exterior and its atmosphere as a whole at twilight, not to construct relationship with viewers. The interview information with the owner of N10 clarified the use of this image. He stated that he was so proud of his in-house dining and he used this image to advertise the hotel dining service. This section focuses on how the hotels and web designers use offer and demand images to create the relationship with viewers. Even though the number of these two types of images is not significant, the findings are consistent with the theory proposed by Kress and van Leeuwen (2006). The next section discusses the characteristics and functions of perspective in advertising hotel facilities and services.

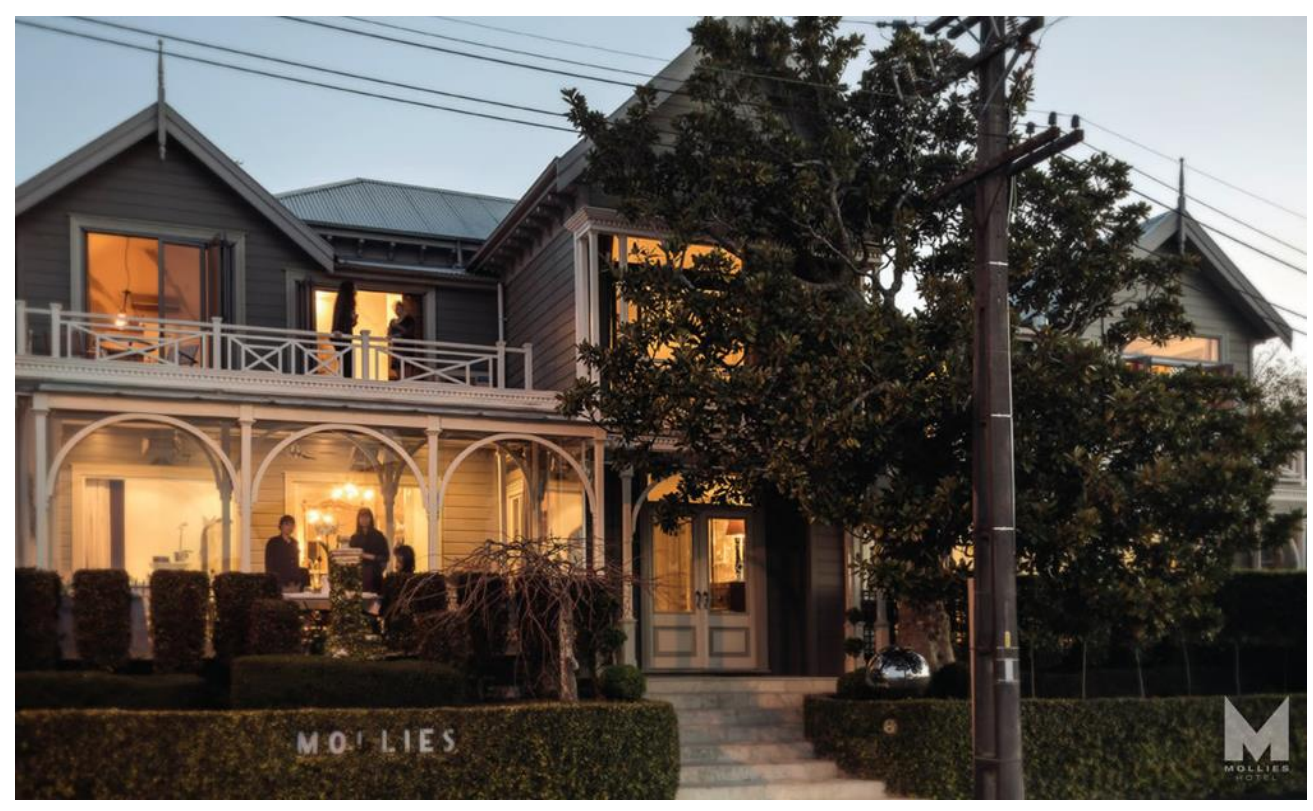

Figure 43: An example of an offer and demand image (NZ10)

Source: $\underline{\text { http://www.mollies.co.nz/ }}$

\subsubsection{Perspective}

As discussed earlier in Chapter 3, section 3.3.1, perspective concerns the relationship between the use of different angles and the involvement between the RPs and viewers. Francesconi (2014), Almeida (2009), Kress and van Leeuwen (2006), and Unsworth (2001) propose that a vertical angle (i.e., high, low or eye level) represents a power differential between RPs and viewers. The position where a camera is placed in relation to the characters can influence the way the viewers perceive them. If the RPs are depicted from a high angle, where a camera is physically higher than 
the RPs, viewers seem to have power over the RPs and the characters look small or weak. In contrast, if the RPs are seen from a low angle, they look more powerful than the viewers. The eye level angle (medium) represents a relation of equality between both sides. Applying the notion of a vertical angle in tourism contexts, Francesconi (2014) suggests that eye level angle implies that RPs in tourism images are friendly and inviting. However, in the context of boutique hotel web advertising, the notion of angle and power (high angle, eye level, low angle) is problematic. One reason is that images in my dataset cannot be clearly placed into the three categories of angles. Many photographs are taken at angles between high and eye level and between eye level and low. They do not have a clear and absolute distinction among three angles. It is more likely a degree or a cline between angles. Another reason is that any power relationship that exists between RPs and viewers due to the angles of the images does not reflect the intention of the hotels' owners. The details will be discussed below. The following section discusses the functions of images that are taken from three different angles.

Tables 39-40 show the interactive meaning based on the use of angle and power relationship (perspective), social distance and involvement (size of frame) between the hotels and the viewers.

Table 39: The total number of images showing interactive meaning depicted on the Thai hotel webpage

\begin{tabular}{|l|c|c|c|}
\hline \multirow{2}{*}{ Angle and power } & High Angle & Medium Angle & Low Angle \\
\cline { 2 - 4 } & 9 & 110 & 14 \\
\hline Angle and involvement & Frontal angle & \multicolumn{2}{|c|}{ Oblique angle } \\
\cline { 2 - 4 } & 36 & \multicolumn{2}{|c|}{116} \\
\hline Social distance & Close Shot & Medium Shot & Long Shot \\
\cline { 2 - 4 } & 25 & 72 & 63 \\
\hline
\end{tabular}


Table 40: The total number of images showing interactive meaning depicted on the New Zealand hotel webpages

\begin{tabular}{|l|c|c|c|}
\hline \multirow{2}{*}{ Angle and power } & High Angle & Medium Angle & Low Angle \\
\cline { 2 - 4 } & 42 & 80 & 3 \\
\hline Angle and involvement & Frontal angle & \multicolumn{2}{|c|}{ Oblique angle } \\
\hline \multirow{2}{*}{ Social distance } & 44 & \multicolumn{2}{|c|}{77} \\
& Close Shot & Medium Shot & Long Shot \\
\cline { 2 - 4 } & 15 & 47 & 67 \\
\hline
\end{tabular}

Regarding the idea of angle and power used in the boutique hotel websites in Thailand and

New Zealand, a medium or eye level angle is the most frequent used (110 images/83\% in Thai dataset and 80 images/64\%in New Zealand dataset). According to Francesconi (2014), a medium shot image is used in order to create a friendly relationship as well as warm welcoming approach between the hotels and potential guests and to show that the hotels are accessible for everyone. Apart from using a medium shot image to amplify friendly relationship, interviews with the hotel owners $(\mathrm{T} 22, \mathrm{~T} 8)$ also explained that the hotels apply a medium angle to represent hotel architecture and suites. All the details of the design and facilities in the hotels and rooms are highlighted and are easily observed by the viewers. For example, Figure 44 portrays the hotel's exterior and atmosphere at night. The hotel uses a medium angle to emphasise the elegance of wooden architectural design which hotel aims to conserve. In addition, the hotel perhaps intends to convey that even though the hotel is a luxury establishment, it welcomes potential guest to experience their personal services. The reason is that medium shot images convey friendly and cosy relationship according Kress and van Leeuwen (2006).

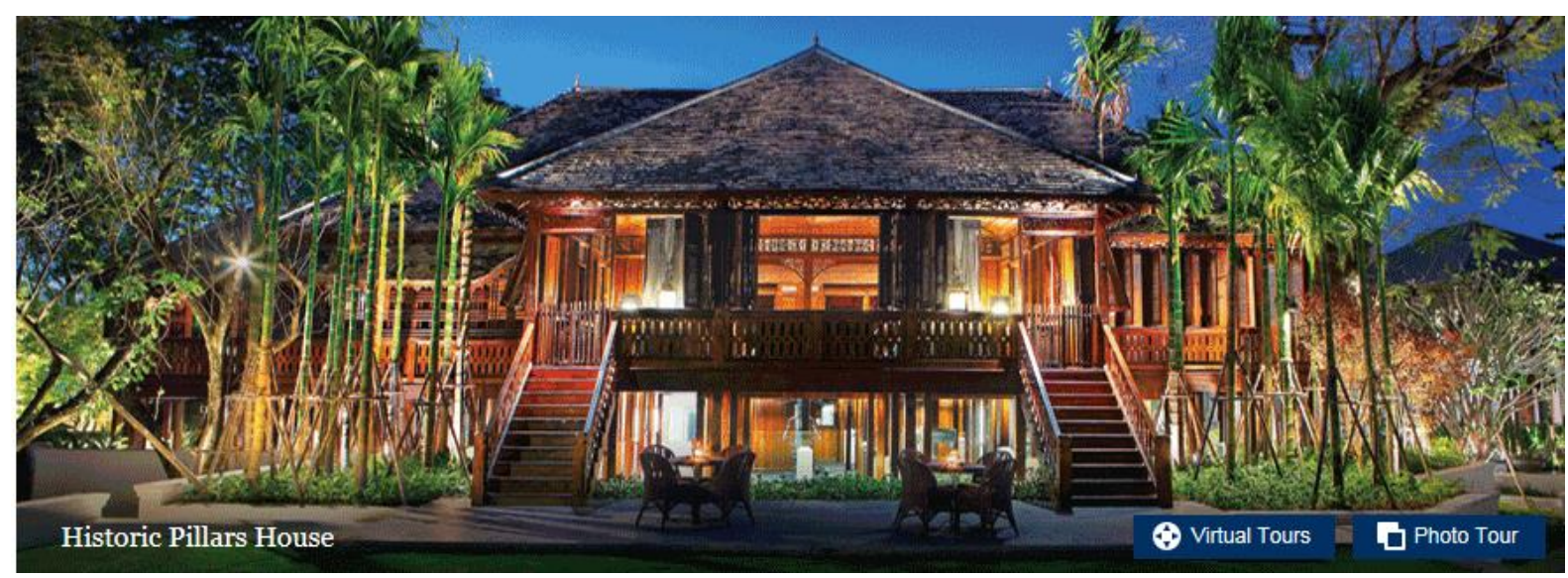

Figure 44: Medium shot image (T22)

Source: http://www.snhcollection.com/137pillarshouse/ 
Figure 45 depicts a hotel suite which is taken from a medium angle. Medium angle enhances the viewers' identification with the hotel architecture and design and allows the viewers to observe every detail of the room design, furniture, and facilities provided in the room. As a medium angle is used to convey the relation of equality, as suggested by Kress and van Leeuwen (2006), accordingly, the possible intention of the hotel to depict this image is to show that this suite is accessible for all potential guests.

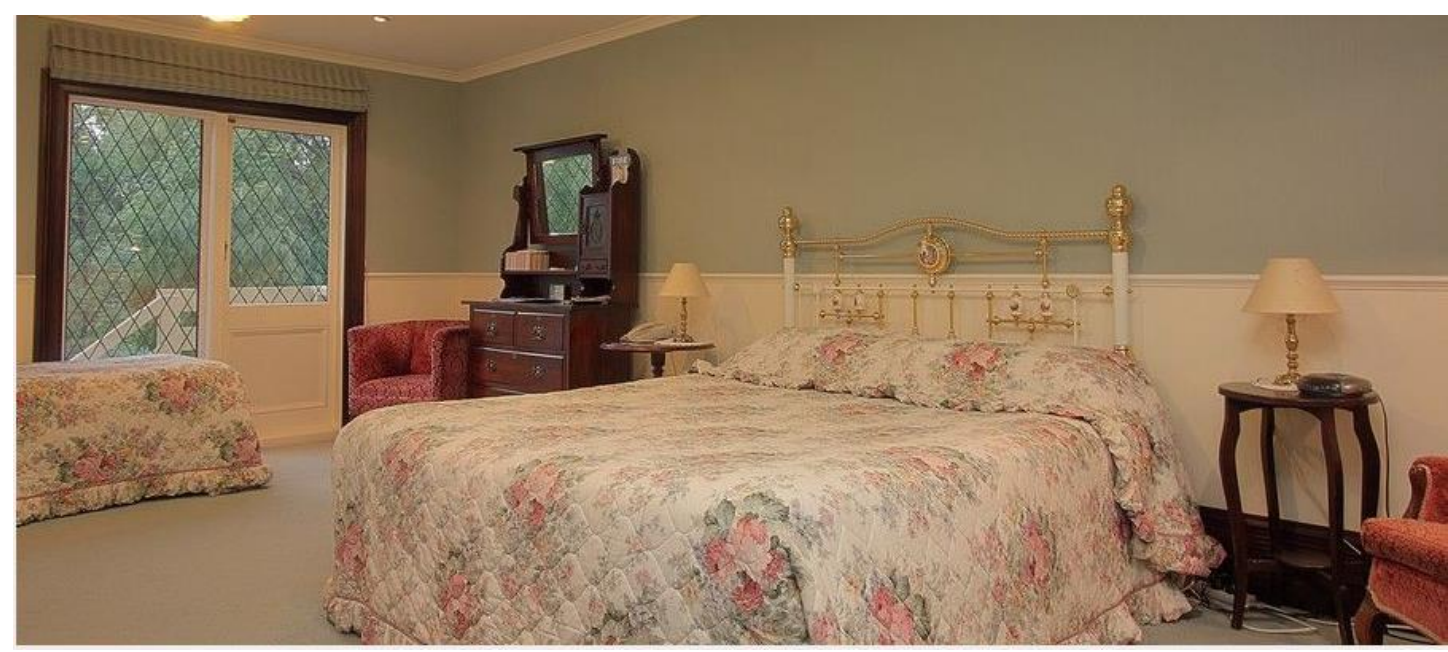

\section{Figure 45: Medium angle image (NZ9)}

Source: http://www.elizas.co.nz/

As shown in Tables 39-40, boutique hotels in New Zealand use a larger number of high angle images (42 images/34\%) than the boutique hotels in Thailand (9 images/7\%). However, there are some problematic cases where some high angle images in the New Zealand cannot be clearly identified as one of the three angles. Certain images are somewhat between eye level and high angle. According to Kress and van Leeuwen (2006), Jewitt and Oyama (2001), Oyama (1999), Unworth (2001), a high angle image encodes high power over the RPs. However, this function does not apply to the images depicted on the boutique hotel websites in my dataset. The interviews with the hotel owners both in the Thailand New Zealand datasets suggest that the use of high angle images is to highlight the hotel architecture and its surroundings as a whole. Interestingly, according to the hotel owner of NZ25, the hotel depicted in Figure 46 intends to promote the impression that it is situated in a good location, secluded and peaceful. A high angle is only a possible way to show that this hotel has beach access. Figures 47 and 48 are pictured from a high angle, more specifically a bird's eye-view in order to show the whole structure of the hotel and its surroundings. Apart from hotel buildings and their hotels exterior, a high angle is also often used to advertise hotel restaurants. Figure 48 shows a table setting. Because it is taken with a high angle technique, this image enables the viewers to see the details of the whole image. Although Kress and van Leeuwen 
(2006) suggest that high angle images imply power relationship between RPs and viewers, in my dataset, high angle images are used to promote and show an entire figure of the hotel buildings and their location.

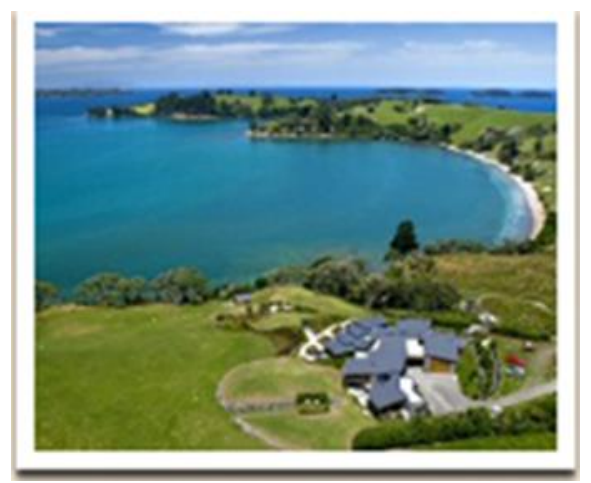

\section{Figure 46: High angle image (T25)}

Source: http://www.waimanapoint.co.nz/

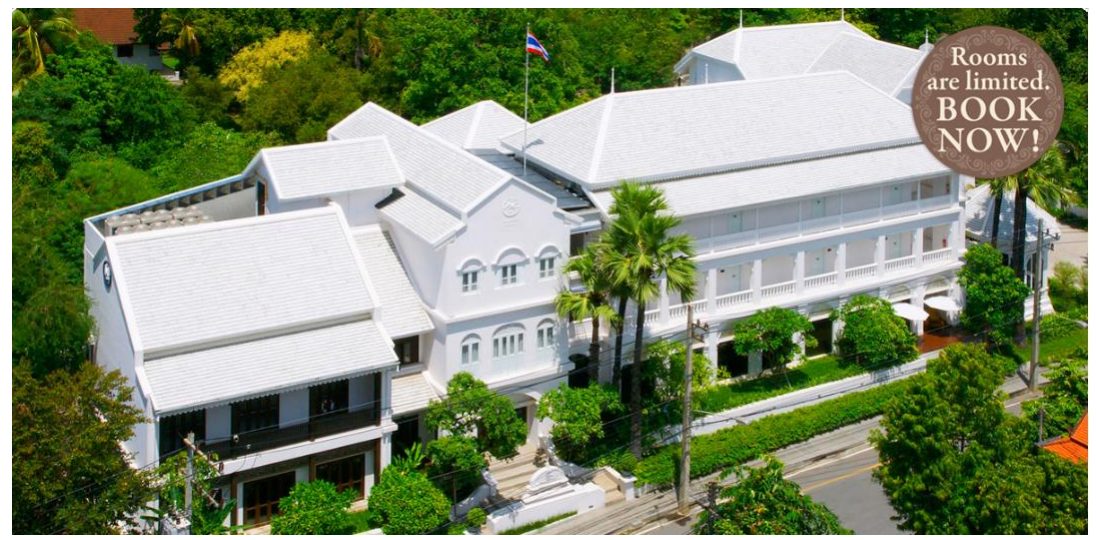

\section{Figure 47: High angle image (T8)}

Source: http://www.pingnakara.com/

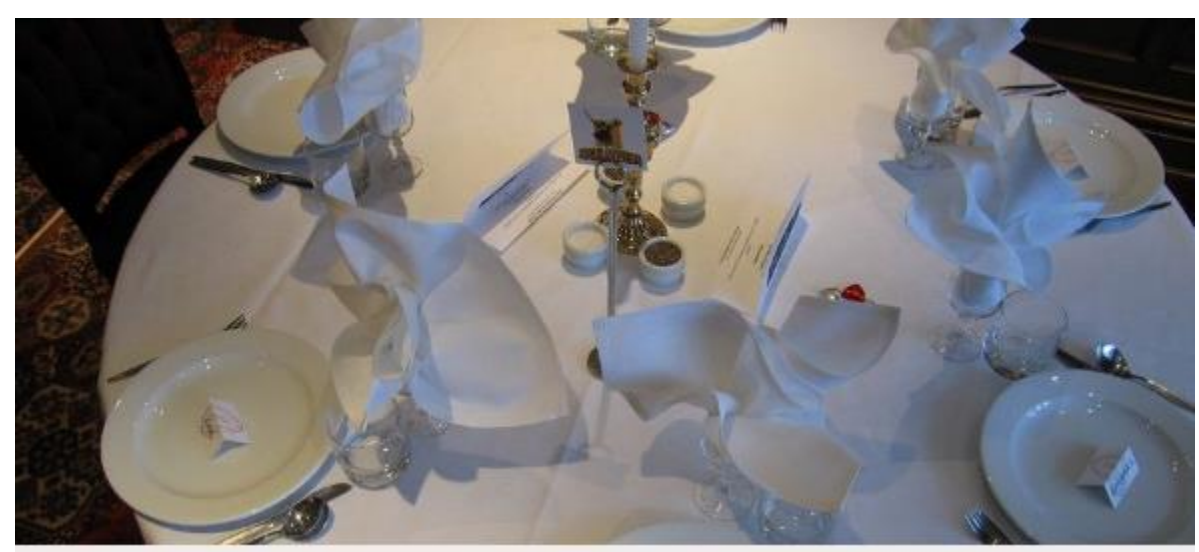

Figure 48: High angle image (NZ9)

Source: http://www.elizas.co.nz/functions-and-events 
In regard to a low angle, the use of lower angle in the Thai boutique hotels (12\%) is more frequent than the New Zealand hotels (2\%) images). However, an application of low angle and its purpose in boutique hotel online marketing is different from magazine advertising. Kress and van Leeuwen (2006) suggest that in magazine advertisements, a low angle is used to portray newsworthy people and celebrities to make them look more powerful than the readers $(2006$, p. 140$)$. In contrast to magazine advertising, boutique hotel advertising aims to promote their accommodation/ services as well as establish a friendly relationship between the hotels and potential guests in order to encourage them to make a booking. Interestingly, Figure 49 is an example that a hotel uses a low angle to promote the design of the hotel. There is no exercising power over the viewers. An interview with the hotel owner (T8) confirms that use of this image of the hotel is employed to show the design of the Lanna Colonial perforated windows style. This section has discussed the reasons behind the use of photographs that are taken with three different angles by the hotels in my dataset. The findings show that the hotels use these images to enhance a structure and details of design and decoration of hotels. This rejects the theory of angle and power noted by Kress and van Leeuwen (2006).

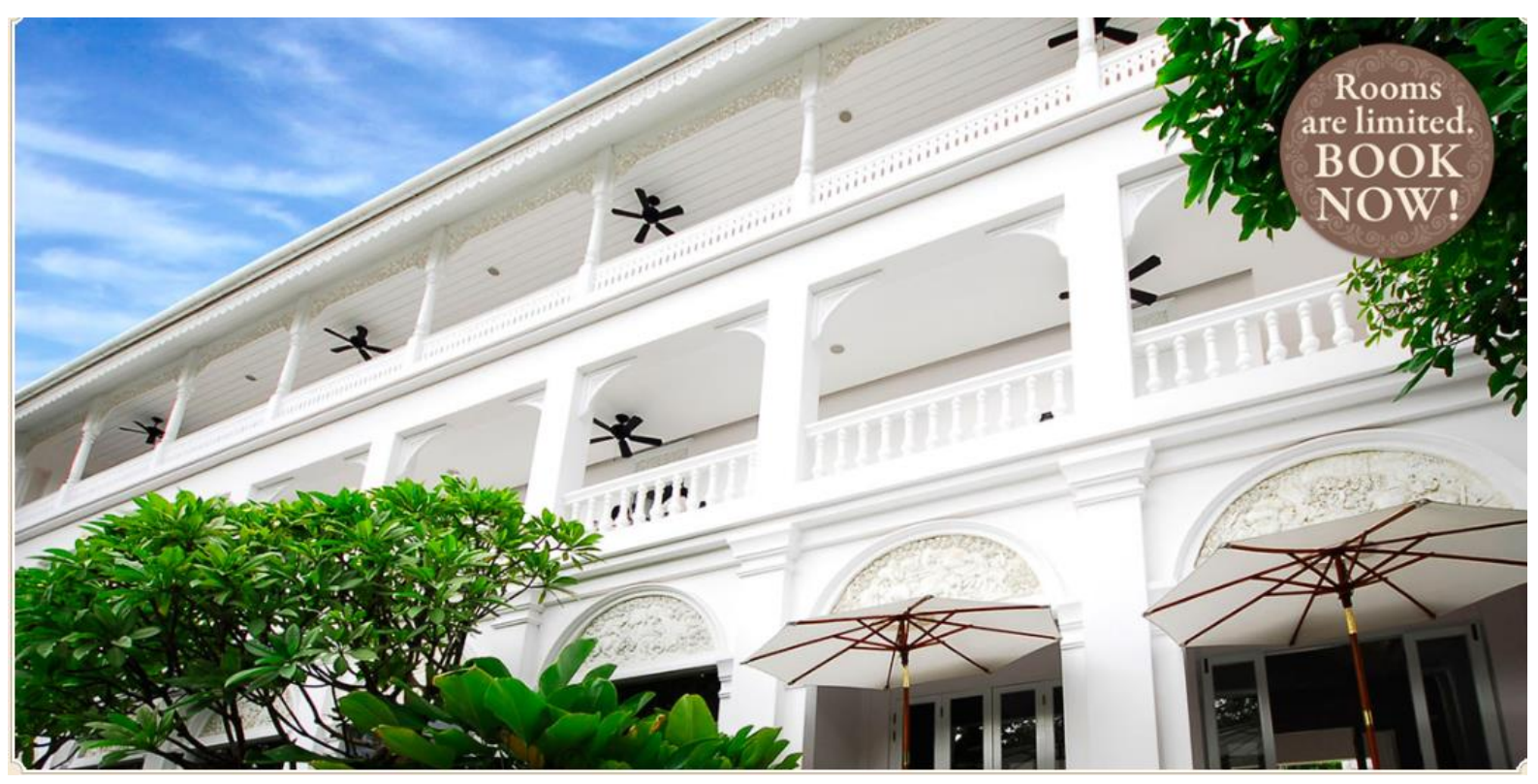

Figure 49: Low angle image (T8)

Source: http://www.pingnakara.com/

\subsubsection{Horizontal angle}

Another way that boutique hotel online advertising discourse is different from the discourse that Kress and van Leeuwen (2006) employed in developing their framework is in the use of horizontal angle. Horizontal angle is concerned with the use of a frontal or oblique angle. According 
to Kress and van Leeuwen (2006), horizontal angle reflects an intention on what kind of relatonship they aim to establish with the viewers. A frontal angle indicates the involvement between the viewer and RPs. It shows involvement, as if the viewers and the represented participants are being involved (Unsworth, 2001, p. 98). They suggest that an oblique angle is associated with a sense of detachment. The viewers are not invited to become a part of the image; they only see how the RPs have been presented. However, the findings are not congruent with this aspect of the framework, suggested by Kress and van Leeuwen (2006); these differences will be discussed in detail later.

Tables 39 and 40 show that the use of an oblique angle (ibid p. 98) is prominent in the boutique hotel online advertising both in Thailand and New Zealand. There are 116 images in the Thai dataset and 77 in New Zealand dataset. Even though the boutique hotels in my dataset advertise their hotels through oblique images more than frontal images, the hotels do not aim to distance themselves or construct disengagement from the viewers. In addition, the use of oblique angle shot is more common in advertising accommodation than a frontal angle technique. Based on interviews with the owners and managers of the hotels (T2, T9, T17, NZ10, NZ9, NZ27, and NZ25), the main reason for the depiction of oblique images is because an oblique angle is to capture the most beautiful spots of the hotels' exterior against the beautiful natural background. In addition, oblique angles are needed to capture suite configurations and restaurant structure.

In both datasets, the depiction of an oblique angle is often used to promote the hotels' exterior, suites and restaurants (Figure 50-52). In Figure 50, the photographer makes use of an oblique angle to capture the side of the hotel structure against the natural landcape in the background. This image aims to highlight the hotel's exterior as well as its outdoor area and a paronamic view of the river and mountain rather than trying to construct detachment from the viewers. Figure 51 is taken at an oblique angle to capture a broader view of the room and provide more detail about the design, decoration, and facilities in the room. Also, an oblique angle appears to make the suite looks more spacious. If this image was taken from a frontal angle, all the details of the room would not be able to captured. In this image, an oblique angle is not associated with disengagement, but it is linked to spatial constraints of a fixed layout of the hotel website template. In Figure 52, the hotel uses an oblique angle to depict the restaurant structure, design, and its atmosphere at night as a whole. 


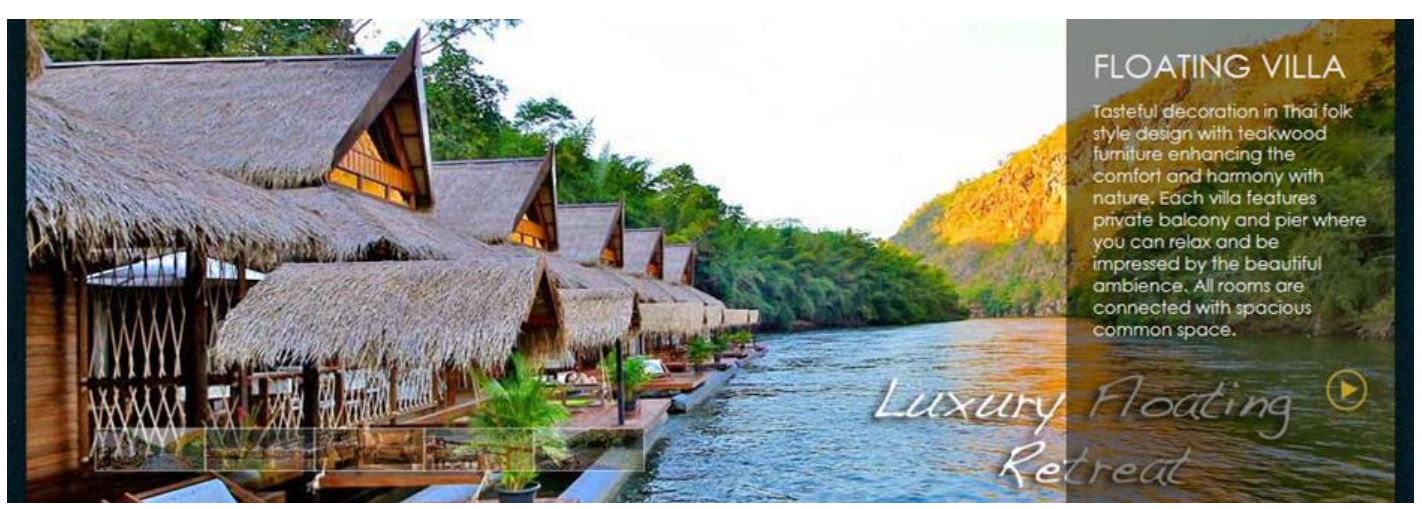

Figure 50: Oblique angle image (T2)

Source: The hotel has updated their website. This image was from the old webpage.

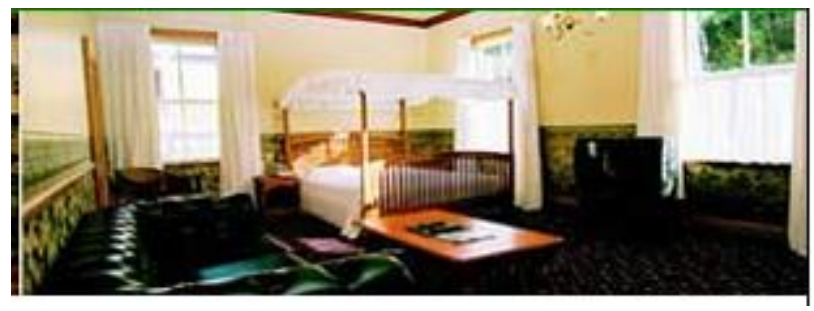

Figure 51: Oblique angle image (NZ18)

Source: The hotel has updated their website. This image was from the old webpage.

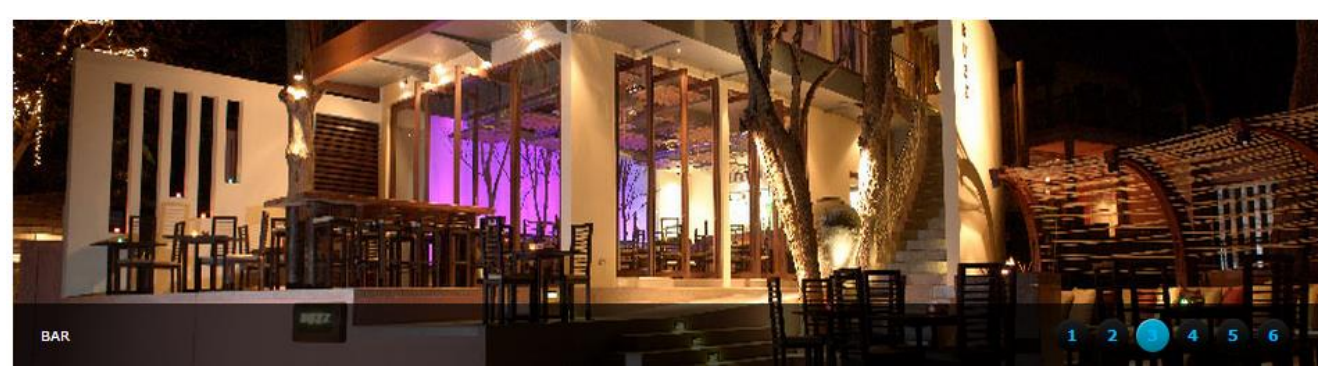

Figure 52: Oblique angle image (T17)

Source: http://www.levimarncottage.com/restaurant-\&-spa

On the other hand, a frontal angle (ibid p. 98) is used to picture restaurants (Figure 53) in the Thai dataset and food (Figure 54) in the New Zealand dataset. Figure 53 makes use of a frontal angle to capture a layout of an outdoor restaurant and its atmosphere at night. The hotel's intention to use a frontal angle in this image is also to highlight that the hotel is located in a good location where the guests can enjoy the view of city and temples along the river while dining. In Figure 54, the web designer depicts this image by using a frontal angle to promote their service on the hotel Function page. The frontal angle allows the viewers to see the details of the dish (colour, texture, and decoration of the food) and arouse the viewers' appetite. This section has described the 
functions and roles of oblique and frontal angle images in my analysis. The findings show that the use of these images in my dataset do not reflect this aspect of Kress and van Leeuwen (2006)'s theory which suggest that these angles imply involvement and detachment between RPs and viewers.

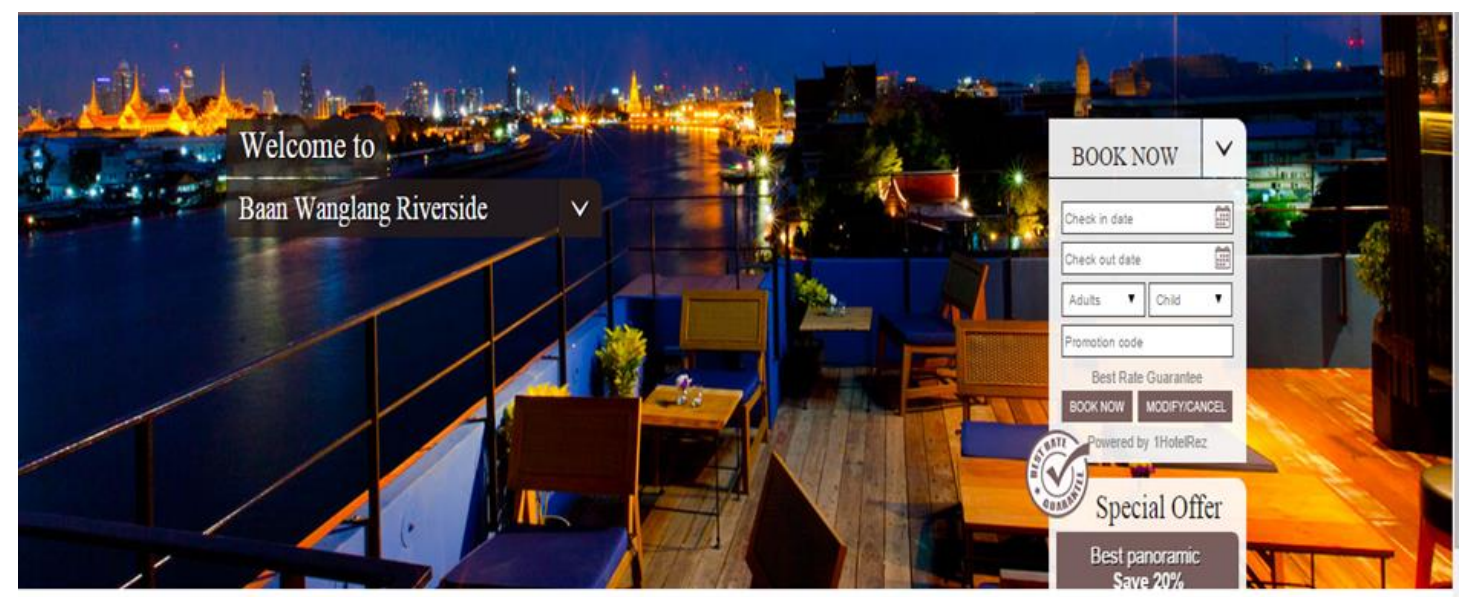

Figure 53: Frontal image (T23)

Source: http://www.baanwanglang.com/riverside_hotel_dinner.php

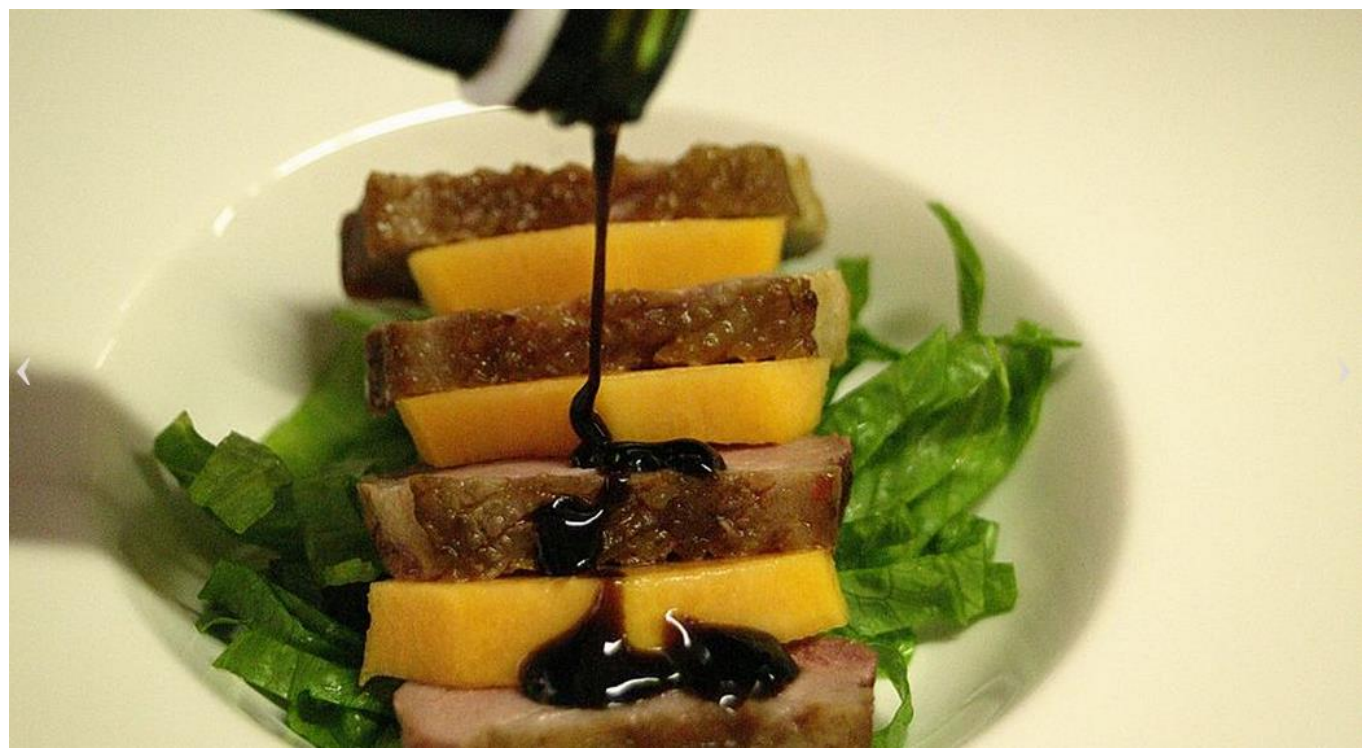

Figure 54: Frontal angle image (T27)

Source: http://www.tewhaulodge.co.nz/cgi-sys/defaultwebpage.cgi 


\subsubsection{Perspective and social distance}

This section shows another problematic issue within the interactive meaning in Kress and van Leeuwen's (2006) model when applying their framework to hotel online promotional materials. The last concept discussed here is size of frame, which is defined by the degree of spatial distance at which RPs are captured in relation to the viewers (Francesconi, 2014; Kress \& van Leeuwen, 2006; Moya Guijarro \& Pinar Sanz, 2008; Jewitt \& Oyama 2001; Oyama, 1999; Knox, 2010; Unworth, 2001). As discussed in Kress and van Leeuwen (2006), close-up shots disclose the individuality and personality of the RPs. The viewers can see every detail of the characters in the image. Kress and van Leeuwen (2006) view a medium shot as more objective and social, while a long shot signals detachment that is usually used with strangers (Oyama, 1999, p. 117). This classification can similarly be achieved in the depiction of objects and landscapes (Francesconi, 2014; Kress \& van Leeuwen, 2006, p. 127). At close distance, an object is depicted as if the viewers are interacting with it; at middle distance, an item is entirely portrayed; while at long distance, a product is fully captured but out of reach. However, the majority of images on websites of the boutique hotels in my datasets are hotel buildings, landscapes, restaurants, and suites as well as their amenities which usually appear in a variety of sizes and shapes. As a result, the analysis of spatial realisation as indicating a level of intimacy between RPs and viewers proposed by Kress and van Leeuwen (2006) for the analysis of hotel online advertising is problematic in some images.

Tables 39 and 40 demonstrate that, predominantly, the visual representations on the Thai and New Zealand boutique hotel websites are dominated by the framing of a medium and a long shot, which realise far personal distance and impersonal distance (Oyama, 1999, p. 209). Whereas a medium shot (Figure 51) is most commonly used to promote accommodation in the Thai boutique hotels (72 images/45\%), a long shot (Figure 56) is the most frequent for hotels in New Zealand to adopt to depict hotels' exterior and their landscape (67 images/86\%). Figure 55 (T8) is seen in medium shot. It allows viewers to witness the patterns drawn on the wall which the hotel owner (T8) aims to match with the colonial period design and colour of the furniture in the bedroom. The intention of such images appears to be to show the readers that the room has a veranda where the guests can enjoy the scenery outside. Because space is limited, long shots do not seem able to depict suite images. Imagine if this photograph (Figure 55) were taken in long shot, the details of the room design would not be highlighted. As a result, viewers would not be able to feel the atmosphere that the hotel aims to create. In addition, if the photo was taken in close shot, the outdoor veranda would be cut off out the frame. A close shot would prevent the viewers from seeing the entire configuration of the suite. Instead of implying a particular social distance between the images and 
viewers, this image enhances viewers' evaluation of the room and its design for them to feel the cosy atmosphere of the room.

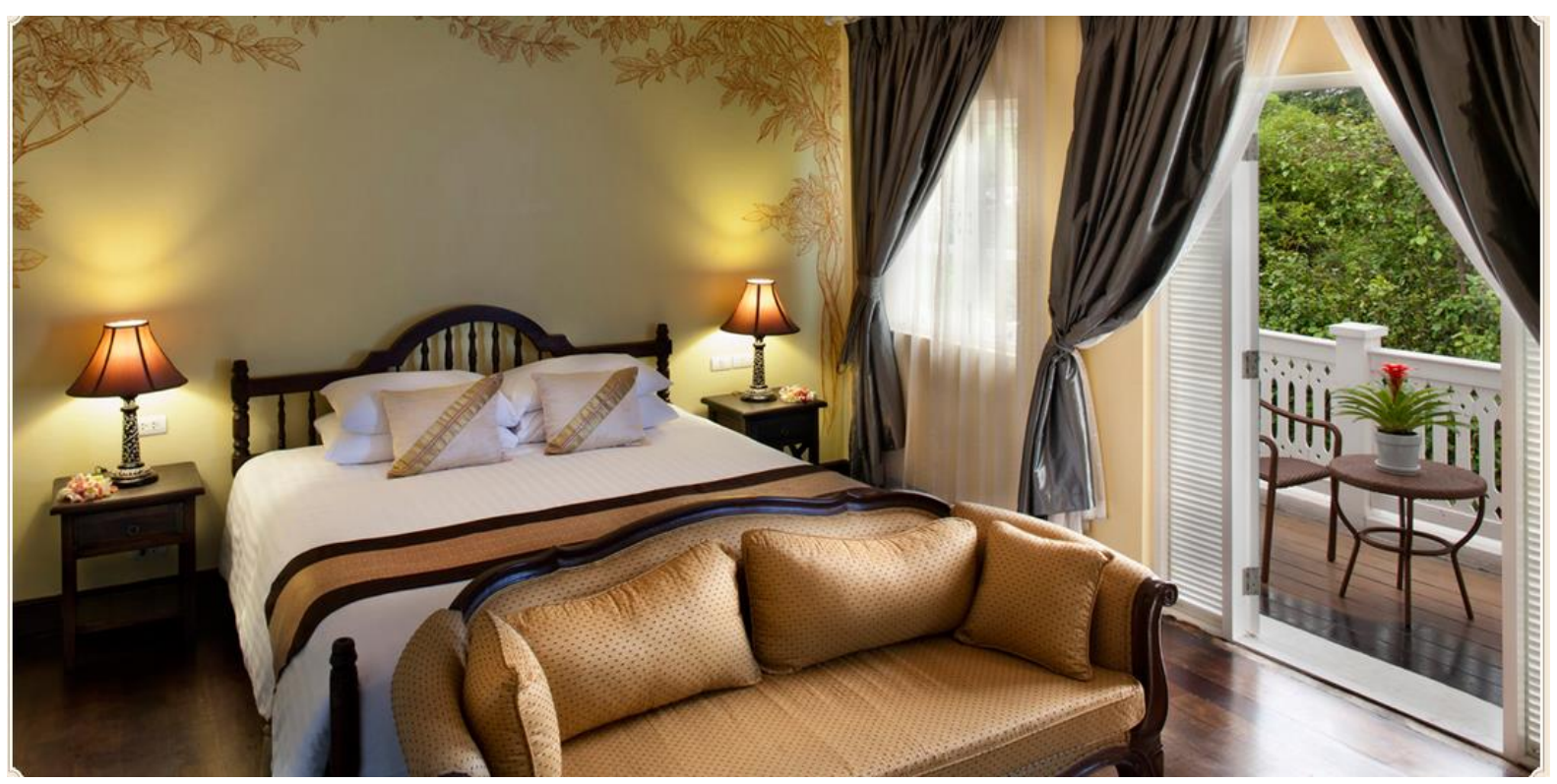

Figure 55: Medium shot image (T8)

Source: http://www.pingnakara.com/rooms

Figure 56 is an example of the use of a long shot, which is the most frequent perspective in the New Zealand boutique hotel dataset. The long shot is mainly used to capture a hotel structure and its surrounding. This image is portrayed full length (long shot), which creates the feeling that the hotel is out of reach for the viewers according to Kress and van Leeuwen's (2006) framework. However, in the context of hotel online promotional materials, Kress and van Leeuwen's suggestion does not appear to be applicable. Even though in the Figure 56 viewers cannot see the hotel in the close distance in order to observe fine detail of the design or decoration, a distant relationship is not the main purpose of the image. Rather, the hotel aims to show the structure of the hotel and its scenery. 


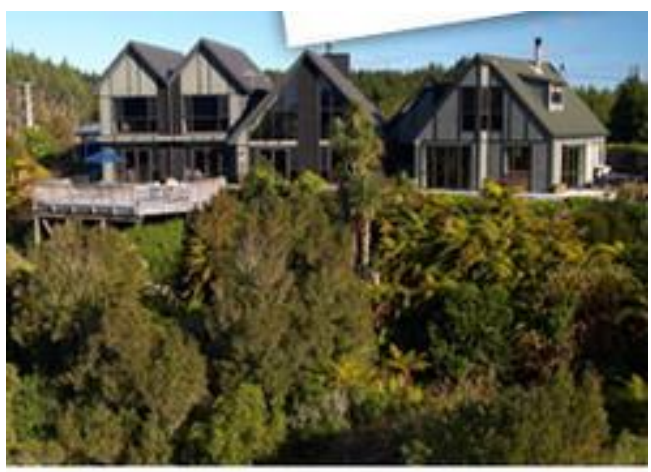

Figure 56: Long shot image (NZ26)

Source: http://www.rimulodge.co.nz/lodge.asp

The last and most interesting shot in interactive meaning is a close shot because it is consistently used across two dataset. A close shot is basically used to capture food in my dataset, as can be seen in Figure 57. The interviews suggest that a close up image magnifies detail, and viewers can see a colour or feel a texture of the food. Also, close shot food images are mouthwatering and encourage viewers to come and use the hotel's restaurant service. The findings of the visual analysis of hotel webpages show that the use of three different types of images - long, medium, and long shot images - are not congruent with the notion of perspective and social distance claimed by Kress and van Leeuwen (2006). The purpose of using such images is to show different perspectives and emphasise the details of the RPs.

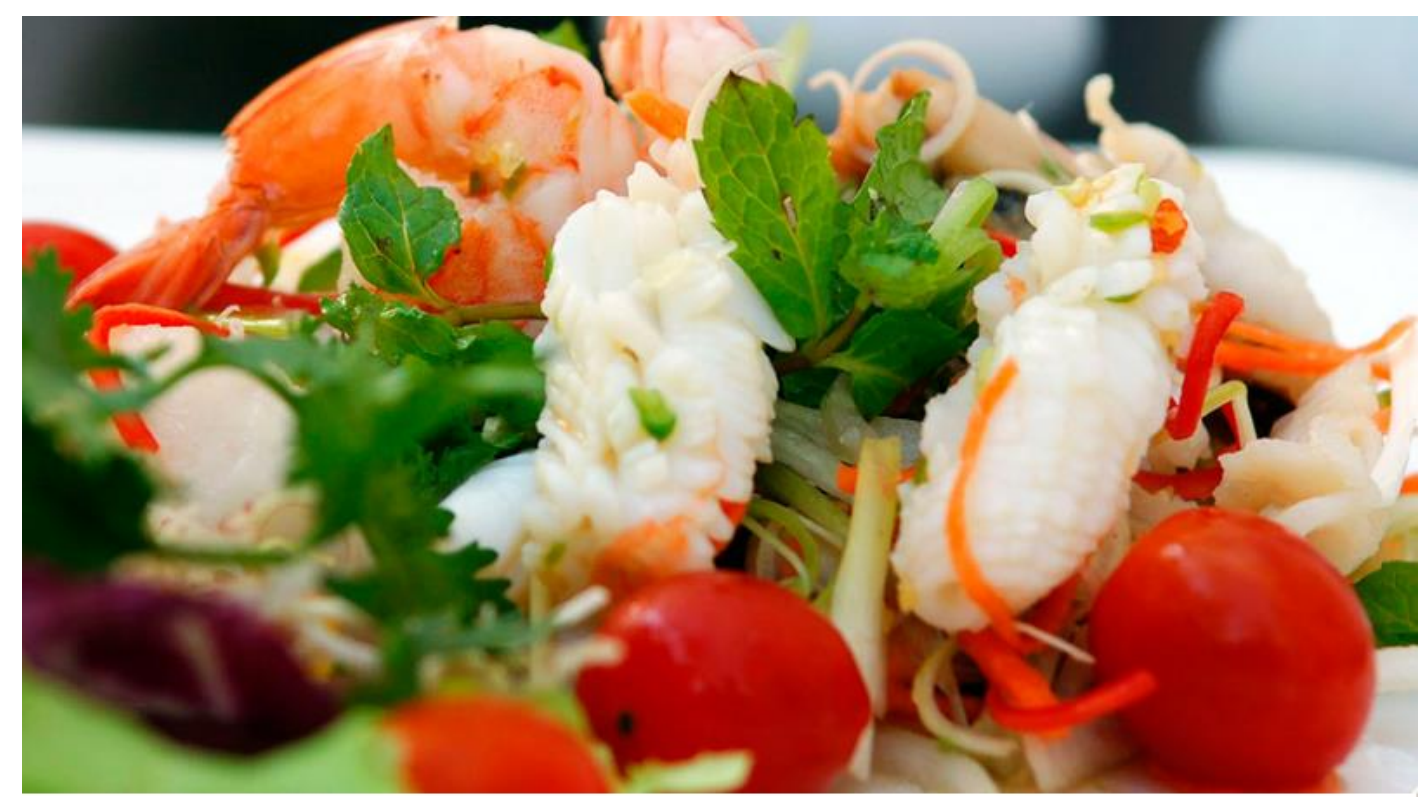

Figure 57: Close shot image (T8)

Source: http://www.pingnakara.com/dining 
As boutique hotel promotional materials have unique objectives and distinctive characteristics, Kress and van Leeuwen's (2006) framework cannot wholly be applied in the analysis of interactive meaning. The relationship between the hotels and the viewers cannot mainly be observed through the use of angle. The interpersonal relation between the images and the viewers also depends on what kinds of objects are represented in the images and the main intention of the hotels that they aim to convey. Interviews with hotel owners are beneficial in this analysis in order to investigate the function of the images. The next section will talk about the last meaning compositional meaning - which involves a structure and layout of webpages.

\subsection{Compositional meaning}

The last meaning is compositional meaning, which is concerned with how the RPs are visually organised in the images. This compositional process can be observed by three aspects: information value, salience, and framing (Kress \& van Leeuwan, 2006; Moya Guijarro \& Sanz, 2008). Organisationally, information value (Kress \& van Leeuwen, 2006), refers to the placement of visual elements in a layout. The left side shows something the viewers already know as familiar content. It is referred to as given (Oyama, 2001 p. 148). The right side represents the new, something which is not yet known to the viewers, therefore, they should pay special attention. The top location implies the most salient or ideal information, and on the bottom refers to real or more specific information (Kress \& van Leeuwen, 2006). The ideal section is usually represented by a visual element, whereas the real is a textual element.

As for information value (Kress \& van Leeuwen, 2006), the ideal section conveys abstract or general information. The textual element providing information about the hotel is placed in the centre, which is the most crucial part of the page. The bottom section provides practical information. In the context of boutique hotels, name, logo, and navigational links are usually situated on the top or ideal section. Beneath the navigation links are where the main welcoming images (banners) depicted in the auto-forwarding carousels are located. They are usually set to automatically rotate (see Figure 58). An auto rotating banner (auto-forwarding carousels) provides slides of rotating images on the top of webpages. It is one of the interactive features of online materials, which is used by the majority of the hotels in both countries, NZ26 being the exception. Interviews with web designers 2 and 3 affirmed that the use of banners is one of the most important and most salient features to attract the readers' attention. This is because viewers' eyes are strongly drawn to those dynamic images. The web designers also explained that the location of the banners is very vital in order to enable users to view the most important content in one spot. Web designer 2 stated that banners should be located in the golden area, which is the most prominent or noticeable place. The 
reason is to avoid banner blindness (Nielsen \& Tahir, 2002, p. 19), where the users tend to fail to notice. In contrast, the bottom or real section of the webpages provides an adress, contact information of the hotel, and information about web designing company; it conveys real or practical pieces of information (see Figure 59). These details encourage viewers to take action and contact the hotels. This practice corresponds to Kress and van Leeuwen's (2006), Unsworth's (2001), and Oyama's (1999) proposition.

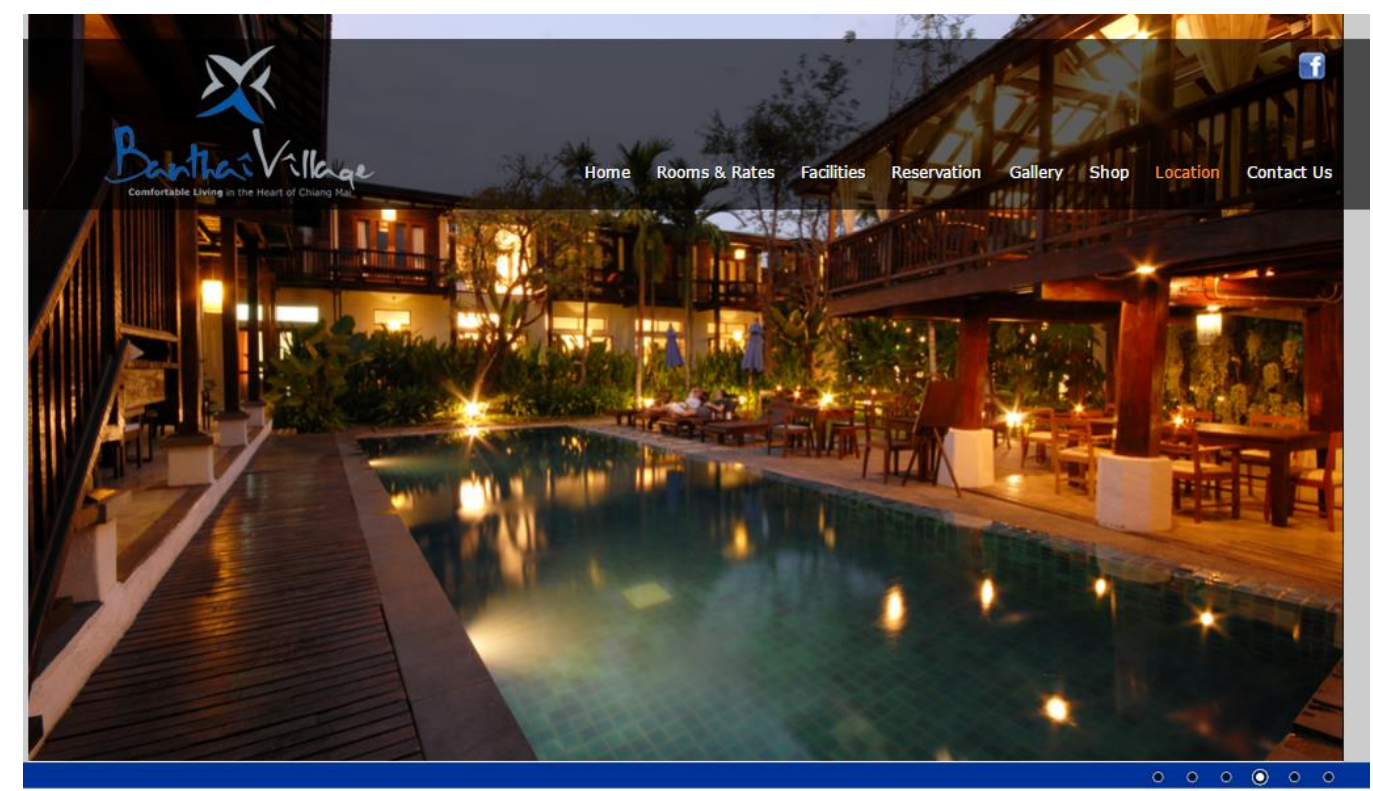

Figure 58: Auto-forwarding carousel (banner) (T23)

Source: http://www.banthaivillage.com/ 


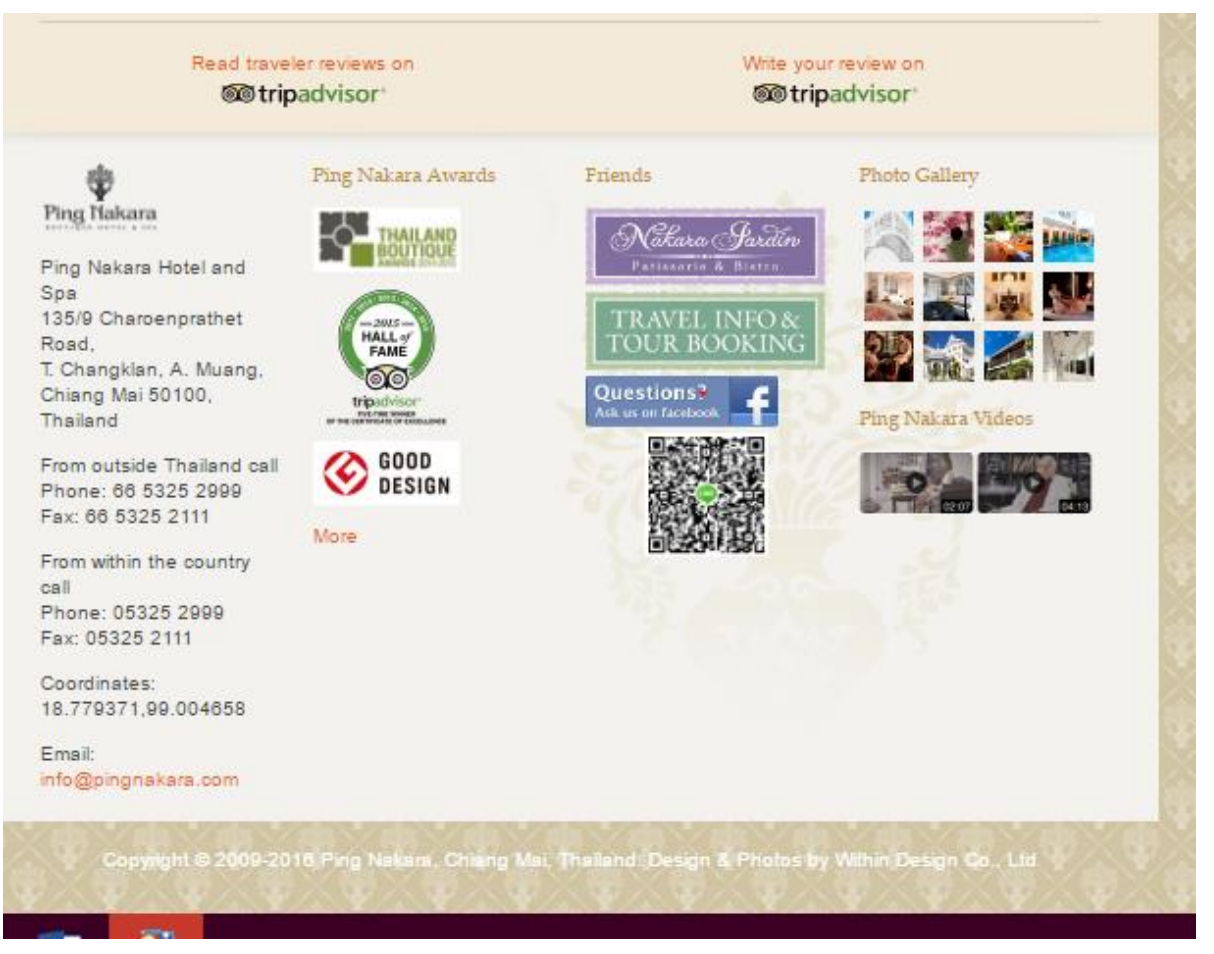

Figure 59: Real or practical information (T8)

Source: http://www.pingnakara.com/

Tables 41 and 42 below show the images representing different features in compositonal meaning presented on the webpages of boutique hotels in the Thai and New Zealand datasets These numbers reflect the trend of webpage design and the differences and similarities between these two countries.

Table 41: The images showing compositional meaning depicted on the Thai hotel webpages

\begin{tabular}{|l|c|c|c|l|l|l|}
\hline \multirow{2}{*}{ Hotel } & \multicolumn{3}{|c|}{ Information value } & Theme colour & Banner & Logo \\
\cline { 2 - 6 } & Ideal-real & $\begin{array}{l}\text { Given- } \\
\text { new }\end{array}$ & $\begin{array}{l}\text { Centre- } \\
\text { margin }\end{array}$ & & \\
\hline T2 & $\checkmark$ & $\times$ & $\checkmark$ & Majestic blue & $\checkmark$ & $\checkmark$ \\
\hline T8 & $\checkmark$ & $\times$ & $\times$ & Brown & $\checkmark$ & $\checkmark$ \\
\hline T21 & $\checkmark$ & $\times$ & $\times$ & White & $\checkmark$ & $\checkmark$ \\
\hline T22 & $\checkmark$ & $\times$ & $\times$ & White and blue & $\checkmark$ & $\times$ \\
\hline T23 & $\checkmark$ & $\times$ & $\times$ & White and blue & $\checkmark$ & $\checkmark$ \\
\hline T17 & $\checkmark$ & $\times$ & $\checkmark$ & White & $\checkmark$ & $\checkmark$ \\
\hline
\end{tabular}


Table 42: The images showing compositional meaning depicted on the New Zealand hotel webpages

\begin{tabular}{|c|c|c|c|c|c|c|}
\hline \multirow[t]{2}{*}{ Hotel } & \multicolumn{3}{|c|}{ Information value } & \multirow[t]{2}{*}{ Theme colour } & \multirow[t]{2}{*}{ Banner } & \multirow[t]{2}{*}{ Logo } \\
\hline & Ideal-real & $\begin{array}{l}\text { Given- } \\
\text { new }\end{array}$ & $\begin{array}{l}\text { Centre- } \\
\text { margin }\end{array}$ & & & \\
\hline NZ10 & $\checkmark$ & $\checkmark$ & $x$ & White & $\checkmark$ & $x$ \\
\hline NZ 18 & $\checkmark$ & $\checkmark$ & $x$ & White & $\checkmark$ & $x$ \\
\hline NZ25 & $\checkmark$ & $\checkmark$ & $x$ & Brown & $\checkmark$ & $\checkmark$ \\
\hline NZ9 & $\checkmark$ & $x$ & $x$ & Blue & $\checkmark$ & $x$ \\
\hline T27 & $\checkmark$ & $\checkmark$ & $x$ & Grey & $\checkmark$ & $x$ \\
\hline T26 & $\checkmark$ & $\checkmark$ & $x$ & Grey & $\checkmark$ & $\checkmark$ \\
\hline
\end{tabular}

My analysis revealed that every hotel in both countries adopts an ideal-real distinction, depicting contrasting elements in the upper and lower parts of the webpages, which is congruent with Kress and van Leeuwen's (2006) framework. The viewers cannot see the whole length of the page at once. The viewers will see the displayed banners first and need to scroll down further to see the rest of the content. This analysis also revealed that cultural differences between the two countries do not influence the way the hotels present their information and images on their webpages. The ideal-real approach is the most conventional layout that the web designers adopt to present information and pictures on their website in both countries.

The next aspect of information value is given-new structure. As for the given (left)/new (right) distinction, as suggested by Kress and van Leeuwen (2006) and Moya Guijarro \& Pinar Sanz (2008), in Western visual semiotic, the left section represents given information that the viewers already know or have viewed and the right section implies new information that needs to be found out. The analysis showed that most of the hotels in the New Zealand dataset conform to the western reading path, such as in Figure 60. The hotels position their photos as given elements on the left and their texts as new elements on the right. The hotels highlight the fact that visual images that are located on the left are familiar elements that guests have previously seen, and textual elements on the right are the offers that the hotel promises to give to the guests when they book the hotel. The overall organisation of the primary content is typically like printed materials, where the texts are horizontally aligned and arranged vertically down the page. Accordingly, based on interviews with the hotel owner (NZ10) in the New Zealand dataset, he explained the reasons why he chose to present their hotel information in the given-new structure. These are because he believes that the given-new approach is a default setting that the majority of audience usually follow. In addition, the 
owner (NZ10) stated that he would like to model their website similar to the way books are generally presented.

In contrast, in the Thai dataset, none of the hotels adopts this given-new strategy to advertise their hotels. The Thai hotels tend to put a banner on the top of the page and follow with textual elements as can be seen in Figure 61. Interviews with web designers $(1,2$, and 3 ) reported that this webpage layout is the more modern design and increasingly popular compared to the given-new approach which appears to be out of fashion in Thailand. One possible reason is that the hotel business in Thailand is highly competitive. Therefore, many hotels hire a web designer to maintain and keep their website up to date. The attention that hotels put into hotel webpage design increases usability of webpages and enables visitors to use the website more easily. These variations in relation to the use of given-new structure between the Thai and New Zealand dataset reflect the differences in the importance of the webpage design in the hotel industry between these two countries.

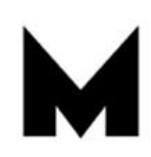

MOLLIES LUXURY BOUTIOUE HOTEL

ABOUT | YOUR SUITE | DINING | EXPERIENCE | SPA | EVENTS | LOCATION | RESERVATIONS | CONTACT

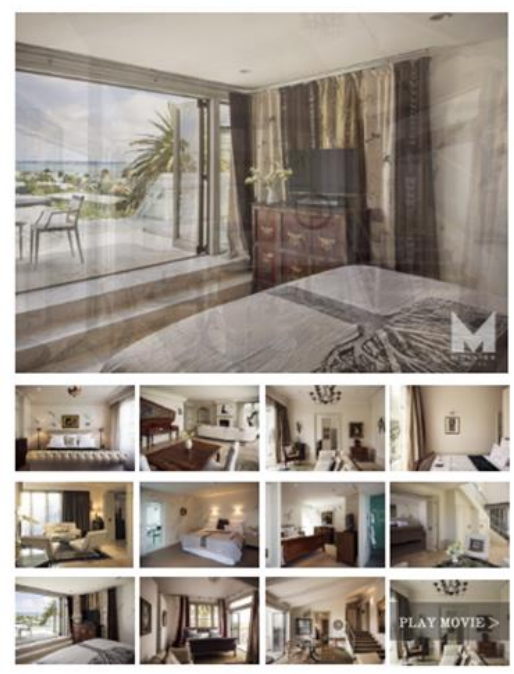
MOLLIES LUXURY ACCOMMODATION Star Boutique Hotel Suites Mollies: ten suites are spacious and beautifully
appointed, with exquisite linens, king size beds and marble ensuites.

The airy, light-filled Junior Suites are the perfect
introduction to the Mollies experience, while Premier Suites extend the luxury with a separate lounge and private balconies where guests can enjoy gourmet a Located on the top floor, the Mollies Suite is the ultimate
comantic retreat. Impeccably furnished to penthouse suite standard. it has two private balconies and panoramic harbour views. It comes complete with its own Study, California king size bed, gourmet kitchenette and oversized spa bath. Guest services and amenities All guests enjoy a superb range of in-suite services and aclities including:

- King size bed with feather pillows and underlay

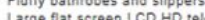
vieving and DVD player - Bluetooth capable sound system - Bluetooth capable sound system
Fully stocked minibar, and tea and coffee making f facilities - In-room safe - Fireplace, (Mollies and Premier suites only)
- Ironfironing board, hair dryer and lugagage rack
- Fresh cut flowers and curated art from the Mollies - A sellection of international magazines

- Nightly turndown service Check availability, book online or make an enquiry
contact us.
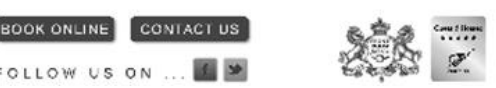

FOLLOW US ON

Figure 60: Given-new image (NZ10)

Source: http://www.mollies.co.nz/ 


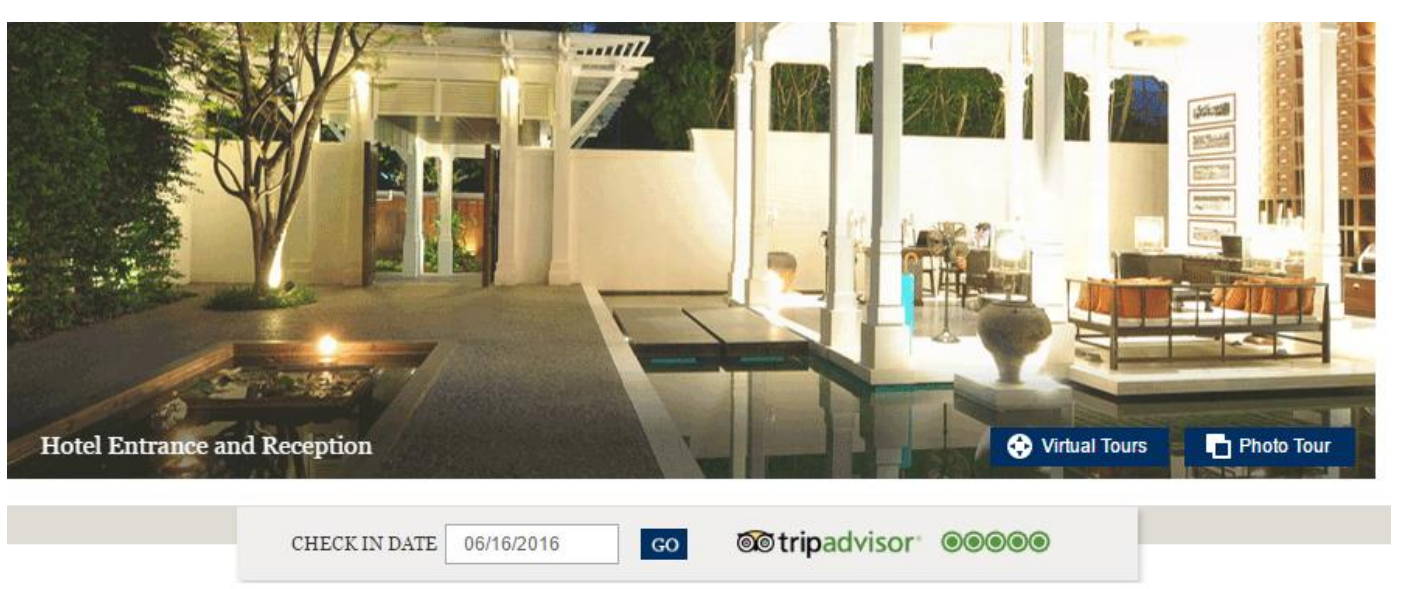

37 Pillars House - Chiang Mai Boutique Hotel

7 Pillars House is a boutique hotel in Chiang Mai that revives the old world elegance of the Orient $t$ offers guests every modern luxury. You are treated as personal guests. As such, we will be moured to lead you through the surrounding charms of Chiang Mai and welcome you back into mpered privacy. The home-style residential concept at our Chiang Mai luxury hotel invites guests to rticipate and enjoy the richness of a like-minded international community while creating plenty of ace for sublime intimacy in sumptuous suites and throughout the tropical grounds.

\section{Figure 61: An example of a hotel that does not use given-new structure (T22)}

Source: http://www.snhcollection.com/137pillarshouse/

According to Kress and van Leeuwen (2006), a centre section represents the most essential part. The visual analysis shows that the centre section, which includes body copy, providing promotional information about hotels and their facilities, reflects the communicative purposes of the hotel. Figure 62 is an example of a hotel that uses centre-margin template representing the visual and textual elements. The layout of the homepage is divided into three main frames in which the top and the middle frames contain the introductory texts about the accommodation and its design. The hotel loads the frame at the center with information about the awards received and a video presenting a story of the hotel. The frame at the bottom mainly shows a photo gallery. Below the bottom frame, the hotel fills the background space with guest reviews, awards received, and social media (i.e. Facebook and Trip advisor). This centre-margin structure is less common compared to real-ideal or new-given structure in my datasets. Only two hotels in Thailand employ this structure to promote their hotels and none of New Zealand uses this template. 


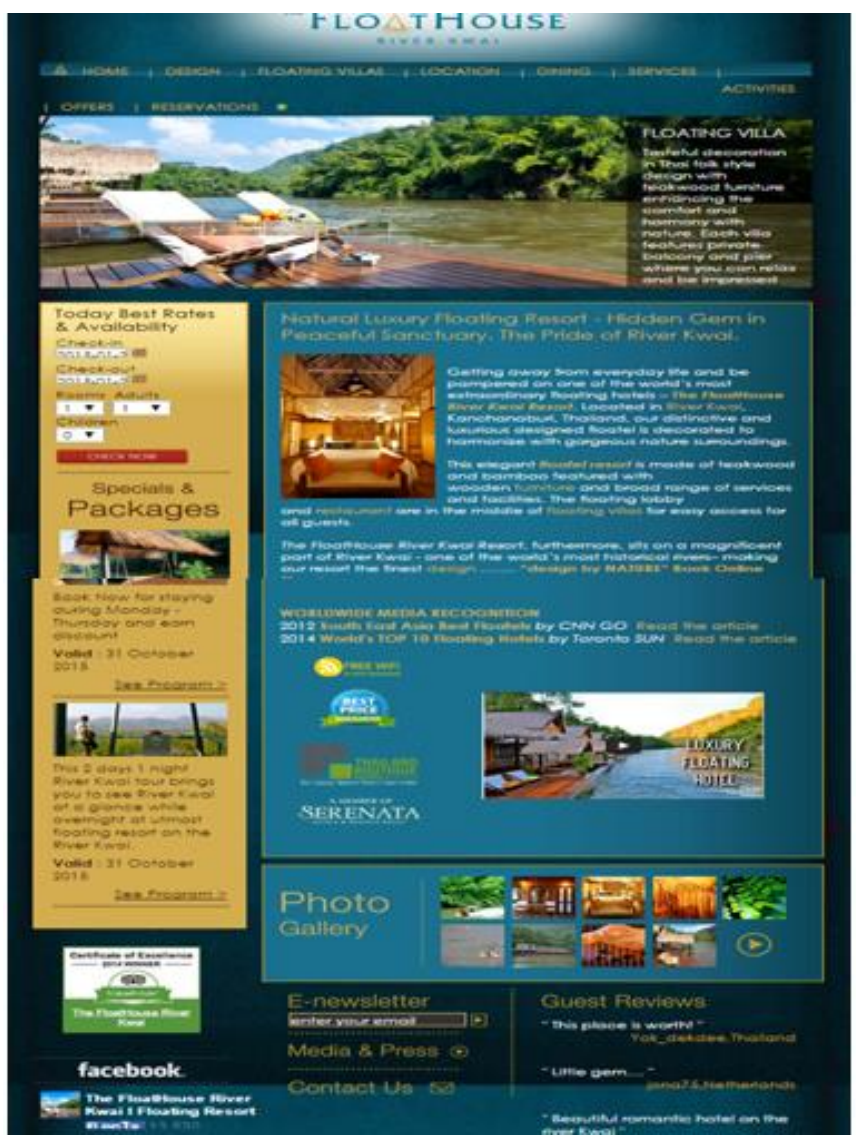

Figure 62: Centre-margin image (T2)

Source: The hotel has updated their website. This image was from the old webpage.

Colour is another important aspect that enhances the power of communication and makes an impact on the perception of the audience. This is because in online advertising, colour is the first thing that potential customers can notice (De Bortoli \& Maroto, 2001). De Bortoli and Maroto (2001) suggest that colour is culturally related. Colours may have different meanings in different cultures. Web designer (1) also supports De Bortoli and Maroto's (2001) statement. He explained that a choice of colour used on website is associated with local culture. He elaborated on this by showing an example of a hotel in the South of Thailand which is located on the beach. The web designer further explained that the owners of this hotel chose purple to promote the hotel because purple represents the beautiful sunset that can be viewed from the hotel. In addition, since some Thais are afraid of being exposed to the sun, the hotel owner believes that purple suits the Thai lifestyle better than bright colour. However, the meaning of purple is different across cultures according a survey conducted by De Bortoli and Maroto (2001). The survey revealed that colour and cultural value are closely related. The study showed that purple is associated with wealth and expensiveness in Asian countries, while purple is connected with cheapness in the United States. 
The use of colour to promote and create the hotels' identity in my dataset reflects the hotels' personal preferences, as can be seen in Figure 62. The hotel uses majestic blue as a background of the webpages. The owner (T2) explained that majestic blue represents elegance, and this idea came from a Prada costume advertisement. As a result, he adopted the combination of majestic blue and gold to enhance the luxury concept of the hotel on the hotel webpages. The use of the color scheme and a singlefont througout the four pages create harmony, visual branding or product identity as proposed by Hiippala (2007, p. 25). According to Hiippala (2007), in order to create identity of a product, a use of consistent layouts of visual and textual elements, colour theme, and typology should be adopted. My study showed that the hotels in both datasets use a similar organisation of texts and images across all three main webpages: Home, accommodation, and Facility. The hotel adopts the same template and structure to present the hotel information and facilities on these three pages to create visual branding or product identity as proposed by Hiippala $(2007$, p.25). Additionally, the information about specials and packages is placed on the left gold frame to create contrast with the majestic blue background. Moreover, on the bottom of the pages, the hotel exploits a black background where the hotel gives contact information, repeats some of the hyperlinks (i.e., Design, Floating Villas, and Dining) and presents additional hyperlinks to inform the viewers more about the hotel and the nearby attractions. The color scheme of the textual elements in this section are also majestic blue and gold, which enhances coherence in the webpages and highlights a luxury concept of the hotel.

My study showed no major cultural differences in the use of colour among the Thai and New Zealand hotel webpages. One distinction is the meaning association with brown between two datasets. Brown is used as a background in a Thai and New Zealand hotel. However, the interviews with the hotel owners revealed that that brown is associated with luxury in the Thai hotel, whereas it is connected with nature in the New Zealand hotel (see Figures 63-64). This example reflects a personal perception of how the hotel owners associate a colour with a particular concept.

Thai hotels generally use colours to emphasise the theme and enhance the design of the websites. However, the interviews with the hotel owners in both countries showed that a dominant theme that is often adopted is a natural theme in both datasets and a cultural theme only in the Thai dataset. The natural theme is highlighted by the integration of a display of natural landscape images and a dominant use of blue, brown, and green. Blue represents rivers and seas. Green seems to be connected to trees and leaves; brown is associated with earth and soil. The combination of brown and green is associated with nature, as suggested by Nodelman $(1988$, p. 61$)$ and this appears to correspond with the theme of the hotels and the interviews information from the hotel owners. One of the hotels in New Zealand (see Figure 63) uses black text on a light brown background to enhance 
the simplicity and elegance of the webpages and create an identity of being a hotel in greenery. The main building of the hotel is brown which is surrounded by trees. Similarly, the use of a dark brown heading and menu tabs displayed on the light brown background of the homepages may reflect the hotel's main objective whereby the hotel owner attempts to construct the atmosphere of being close to nature for the viewers.

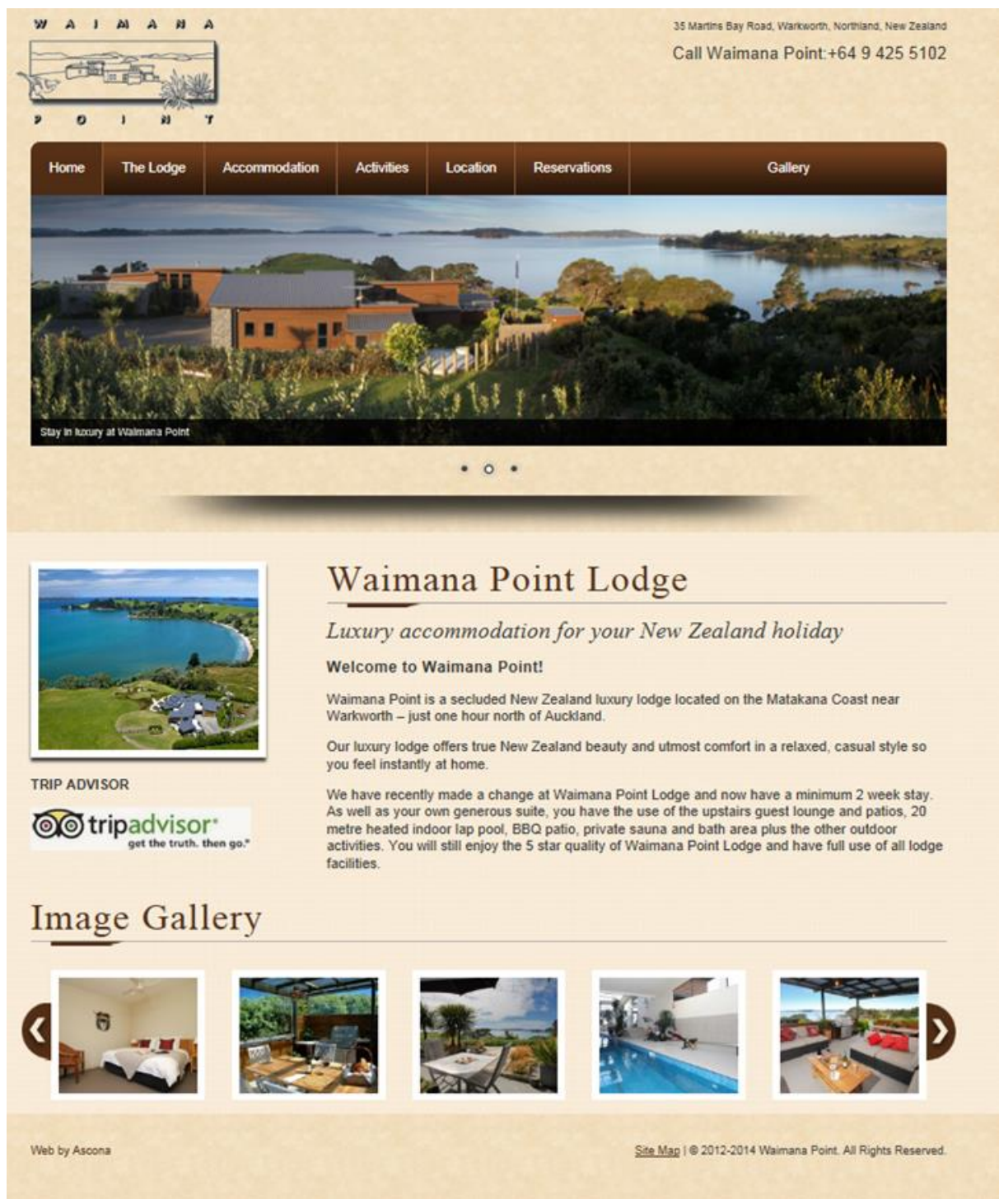

Figure 63: A natural theme (NZ25)

Source: http://www.waimanapoint.co.nz/ 


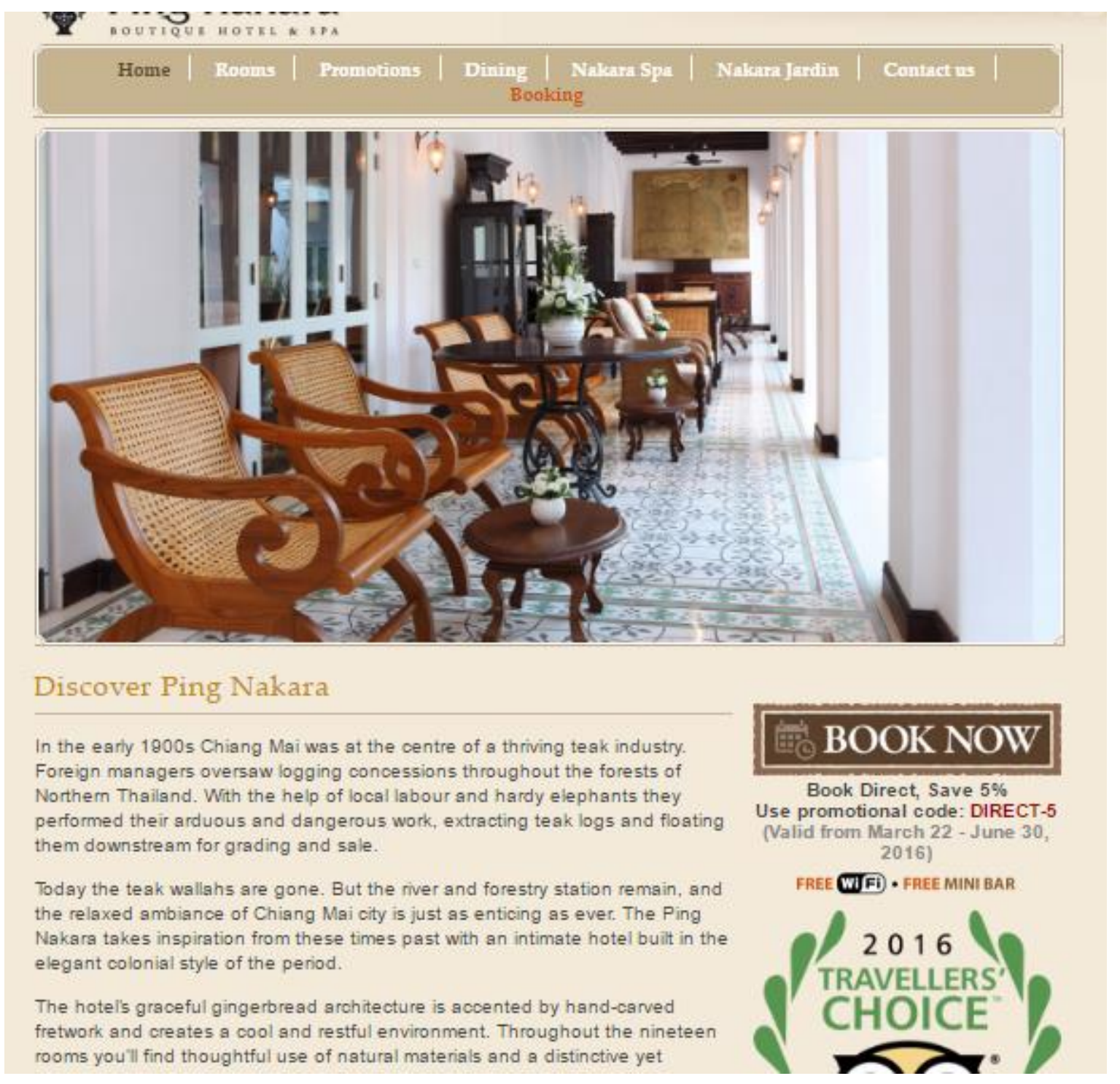

Figure 64: Luxury theme (T8)

Source: http://www.pingnakara.com/

Apart from the natural theme, the importance of cultural value is also highlighted in the Thai hotels. This is evidenced by the use of hotel architecture, Thai art, and Thai lifestyle images. The emphasis on the core value of the Thai culture through a presentation of the hotel websites correspond with Sigala and Leslie's (2006) suggestion that the hotel industry is an integral part of the cultural tourism product. According to Sigala and Leslie (2006), boutique hotels are one of the distinctive examples of cultural tourism products. This is due to the fact that travellers who are interested in exploring art, culture, and history tend to select small- scale historic boutique hotels as their priority accommodation. 


\subsection{Conclusion}

The visual analysis of the three metafunctions - representational, interactive, and compositional - reveals that there are certain problematic aspects that these metafunctions cannot be entirely applied to hotel online promotional materials. The most problematic area lies in interactive meaning, which concerns a relationship between RPs and viewers. However, my findings show that the use of interactive meaning is not associated with power relations, distance, and involvement between RPs and viewers. Rather, the role of these type of images is to enhance the identification of hotel structure and design. The use of three different angles -close, medium, and long - are used to show the different services and facilities offered by the hotels. That is to say, Kress and van Leeuwen's (2006) model should be expanded to cover the hotel and tourism context.

In regard to the distinction between the use of visual elements to promote hotels in Thailand and New Zealand, the anaysis showed that the major difference occurs in compositional meaning - webpage layout. The reason is that the hotel business in these two countries has a different focus. For Thai hotels, the owners and web designers believe that in order to compete with other hotels, hotel websites should be up to date and easy to use in order to attract viewers' attention. On the other hand, New Zealand hotels pay more attention to building personal relationship with viewers. This also reflects the different scale in the hotel business between these two countries. 


\section{Chapter 8: DISCUSSION}

This chapter discusses the main findings and a comparison between the present findings and the findings in previous studies. It attempts to give reasons for the similarities and differences between the presentation of textual and visual elements of the Thai and New Zealand hotel webpages.

\subsection{The purpose of hotel webpages}

The introduction of the Internet creates a new platform of communication in online promotional genres, in which images and texts co-occur and work together to create meaning. The effectiveness of hotel webpages relies on several elements working together. Often, the image and text are interdependent.

The main purpose of my study is to investigate boutique hotel webpages as a promotional genre. I pay attention to the characteristics of promotional texts and images as well as their functions. I also examined the influence of culture on the representation of textual and visual elements between Thai and New Zealand hotel webpages. Genres, as we have come to understand them regardless of the genre traditions, are purposive and goal directed. Focusing on the presentation of textual and visual elements, the main purposes of the hotel webpage genre are to inform potential customers about the facilities and services. At the same time, hotels aim to build a positive image, engage with potential customers, and persuade them to take action (Bhatia, 1993). These main purposes are manifested through a combination of verbal descriptions and visual design presented on the hotel webpages. These informative and persuasive purposes are in line with the work on promotional genres by Bhatia $(1993,2005)$ and Virtanen $(2005)$, even though their work did not focus on online advertising. This is because institutions usually adapt a new channel of communication to suit their main purposes (Djonov \& Knox, 2014). This suggests that the aims of communication are more important than the format or medium of communication because in order to promote products or services, the aims have to be recognised by the target group.

\subsection{Characteristics of hotel webpages}

The findings of my study reflect the core value of genre theory which is based on highly conventionalised communicative settings that influences the similarities in word choices, selection of specific visual images, web layout, and design in promoting the hotels. In order to advertise hotels online, web designers combine all of these elements to make their webpages informative and 
persuasive, which are essential values in promotional genres. Genre itself is one of the influential factors that govern the way the web designers write hotel webpages. The analysis allows us to understand how web designers construct their generic knowledge and exploit their creativity to meet the demand of promotional genre conventions and serve the communicative purposes within the genre. The details of how web designers use language and select images as well as web design to promote their hotels is discussed in the next paragraph.

In regard to move structures, the hotels in Thailand and New Zealand adopt three obligatory moves, which contain the main communicative purposes that are vital in order to structure the boutique hotel webpage genre. The terms obligatory and optional are used variably in genre studies. Since the criteria to identify the typicality of moves are not clear-cut, previous genre literature has established its own principles to justify whether moves are obligatory or optional. Some studies (i.e. Kanoksilaphatham, 2005; 2007a; 2007b) explain explicitly how they operationalise the frequency. However, some studies (Bhatia, 1993; Swales, 1990) leave the readers of their articles to derive the meaning of obligatory from their frequency tables. In my study, I have chosen Mulken and van der Meer's (2005) move demarcation, in which they propose that moves that occur in $75 \%$ or more of text are obligatory moves, but they are optional if they occur in less than $75 \%$ of text. The first obligatory move is the Move: Describing the rooms. It is the most important move because it occurs in $100 \%$ of texts in both datasets. Since the primary focus of advertising hotels is to encourage the potential guests to make a booking and come to visit the hotels, Move: Describing the rooms contains the most distinctive promotional message that is used to attract the readers'attention. The second most frequent move is Move: Introducing the hotel/facilities/services, which is found in $100 \%$ of texts in the Thai hotel webpages and $90 \%$ of texts in the New Zealand hotel webpages. The main purpose of this move is to introduce the name/or slogan of the hotels, to welcome the readers, and to provide a heading for different sections of the hotel facilities and services. An additional purpose of this move is found in Johari and Ali's (2015) work. They discovered corporate websites use welcome notes to make customers feel comfortable and at home. The third most important move is Move: Establishing credentials. It occurred in $78 \%$ of texts in the Thai dataset and $75 \%$ of texts in the New Zealand dataset. This move was originally described by Bhatia (1993) in his sales promotion letters. The purpose of Move: Establishing credentials in my dataset is consistent with the communicative function proposed by Bhatia (1993). The purpose of this move is to construct a positive image for the hotels and to convince the readers that the hotels are well-established with outstanding and unique services and facilities.

Even though move structures are realised and governed by the main purposes of promotional genres (e.g. to inform and to promote the facilities and services), there are subtle 
variations between the Thai and New Zealand datasets. However, these differences are not considerable and do not change the main purposes of boutique hotel online advertising genre. The differences are the purposes in the application of Move: Describing offer and Move: Calling for action between the two datasets. The Thai hotels exploit Move: Describing offer to inform the readers about the facilities and services provided by the hotels. In contrast, the hotels in the New Zealand dataset do not use Move: describing offers as often as the Thai hotels do. Rather, the New Zealand hotels use Move: Calling for action more often than the Thai hotels to put more emphasis on encouraging the readers to initiate further action and contact. The factor behind the differences in the use of these two moves is not from cultural differences as I have anticipated but it is because the boutique hotels in Thailand and New Zealand have a different marketing focus when advertising their hotels. One possible explanation for the lower frequency of the Move: Describing offer in the New Zealand compared to the Thai dataset may be because the hotels in the New Zealand dataset are generally smaller in scale than the hotels in the Thai dataset. The New Zealand hotels are mainly operated by hosts, who are a couple. Supporting my claim that hotels in the New Zealand dataset are smaller than the Thai hotels is the fact that four out of five New Zealand boutique hotel owners that I interviewed had converted their property into boutique hotels and the hosts had a separate residential section for themselves within the same properties. The minimum number of rooms in the New Zealand hotels is four rooms while in the Thai hotels the minimum is nineteen. In addition, the New Zealand hosts normally do all kinds of work in the hotels as can be confirmed from my interview with the hotel owners of NZ10 "I do everything from running the hotel to washing dishes."

Another piece of evidence supporting the fact that the differences in rhetorical moves between the Thai and the New Zealand webpages are not from cultural variation but from a difference in marketing focus is that the New Zealand hotel are opened seasonally and with conditions. Conversely, most of the Thai hotel owners built the hotels for tourism and commercial purposes. The management team often uses Move: Describing offers to emphasise the offers and benefits that visitors will receive when advertising their hotels.

Apart from the difference in marketing strategy between the hotels in the two countries that causes the variation in move structures and their frequency of occurrence, I argue that the process and the practice of developing the webpages between these two datasets are also reasons why move structures between Thai and New Zealand hotels differ. The participants from both countries explained the procedures that they adopt when producing their hotel webpages. They described that there are a variety of options. The first option is that the hotels produce their promotional texts by consulting other hotel websites that have a similar theme as a model for writing their websites, and 
then they give web designers the texts and photos that they would like to put on their websites. This technique is used by some of the hotels in both datasets and this may influence the similarities in move structures across the two countries.

The next option of designing websites is explained by one of hotel managers in Thailand. This practice is suitable for the hotels which have larger budgets and aim to increase their customer base from e-marketing. The hotel manager of T22 stated that in order to develop an effective promotional message, the hotel manager hired a professional copywriter and let her stay at the hotel at least for a few days or a week to write it. The hotel manager explained further that he believed that the copywriter would be able to describe the hotels more accurately if she stayed at the hotel. This approach enables the copywriter to portray the characteristics of the hotel more knowledgably. The copywriter is able to discuss and brainstorm the ideas with web designers and the hotel management team.

A third option of website development was revealed by two of the Thai web designers who were interviewed. Web designers 1 and 2 reported that the hotels hire professional web designers and copywriters to write an entire website for them. Web designer 2 explained that in order to develop English webpages, they write promotional texts in Thai first and then send those copies to the translation department, where the translators specialise in tourism texts. I argue that these three different approaches that the hotels use to develop hotel webpages in the Thai and New Zealand datasets influence the differences and similarities in the way the web designers across these the hotels in these two countries use language and images to promote their hotels

As I discuss further in Chapter 9, this is of relevance to my research question concerning move structures that occur in the Thai and New Zealand hotel webpages. The move analysis shows that the hotels in both countries conform to certain structures and rules when developing webpages. This is evidenced by the similarities in organisational structures across the two datasets. These findings support the notion of conventionality within the same genre, which is proposed by Bhatia (1993), Hyland (2000) and Mauranen (1993). These scholars suggest that genres are socially realised by the members in a discourse community and are governed by certain conventions showing that they belong to a particular genre. The minor variation across texts within the same genre seems to be an innovative use of language of expert members rather than a different genre. My analysis also proved that cross-cultural aspects play a subtle role in hotel online advertising genre which contradicts Crossley's (2007) suggestion that moves are culturally dependent. When a writer travels between cultures but remains within the same genre, Crossley (2007) suggests that move expectation of that genre may change, but this is not the case in hotel online advertising. 
share two similar methods and processes in producing their webpages, the promotional materials that are presented on their hotel webpages contain more similarities than differences across two countries. It reflects that even though the texts are produced in different countries, they share distinctive similarities. This is because the hotels in both countries follow the same norms, and have similar purposes and a similar target audience. The above discussion reveals that cultural differences between Thailand and New Zealand are not the main contextual features determining the variations between the two datasets.

As discussed in section 3.1 Chapter 3, ESP genre analysis serves the essential role of helping novice writers gain insight into textual conventions of a genre by focusing on its communicative purposes (Bhatia, 1993; Hyland, 2009: Swales, 1990). This is because genre analysis provides textual structures and linguistic resources which can contribute to teaching and learning writing. The regularities of lexco-grammatical features within a genre are determined by the usage of experts of the discourse community. In addition to the use of move structures, the use of persuasive and distinctive linguistic features is also important to achieve the purposes of hotel webpages. Genres are resources for meaning which enable novice web designers to select core linguistic features that are associated and feed into the communicative purposes of hotel and tourism discourse.

Since the hotels in Thailand and New Zealand have similar social purposes to advertise their hotels, they also share some similarities and differences in the use of linguistic resources to attract the readers' attention. Consistent with the core notion of genre theory, the findings of my register analysis revealed that the use of linguistic features in hotel webpages in Thailand and New Zealand conforms to "conventions" or "regularities" of promotional genres. The first linguistic feature is pronouns, which are used in promotional texts to build a relationship with potential guests. This is confirmed by the use of the frequent pronouns (our, we, you, your) in my study. These pronouns are used in the interaction between the hotels and the readers. The main purpose of the use of these pronouns is to engage readers with the hotel promotional texts. This may be because hotel web advertisement is an interaction between two parties: hotels and target guests. As a result, the hotels convince the target guests that all of the facilities and services they offer are crafted for their particular needs and benefits, and persuade them to visit the hotel. Additionally, the interview data from the hotel hosts in New Zealand confirms that they used pronouns as a persuasive tool to directly address and connect with the readers. My analysis is congruent with Janoschka's (2004) and Samaru-Charles's (2001) work, that pronouns are a linguistic strategy of persuasion that is widely used in advertisements in order to have a persuasive impact on the audience. 
Hotel webpages use many adjectives to create emotional appeal and describe the characteristics of hotels, facilities and services. This is evidenced by the use of boutique to describe hotels as the web designers aim to differentiate their hotel from other kinds of hotels, especially chain or box hotels, and to cater to the tourists who have shifted from chain or 4 and 5 star hotels to more intimate accommodation. Another interesting linguistic feature is imperatives which is used to achieve several purposes. Relevant to Hofacker and Murphy's (1998) and Janoschka's (2004) in terms of the function of imperatives used in online advertisement, my study also shows that web designers use imperatives to instruct users and initiate an action. They are not employed as offensively as a strict command; they rather perform a polite request. These instruction verb structures are frequently found in Move: Calling for action, where the main purpose is to encourage readers to initiate certain action. Examples are, Contact, Click, Book, Read, and Check out.

The way the hotels use persuasive language to attract the readers' attention shows certain variations. These variations represent the boutiqueness and the way the hotels position themselves as a boutique hotel differently between two countries. Since there are no major differences in the use of linguistic resources between the datasets of these two cultures, I argue that the culturally specific features between both countries do not determine the way the promotional language is used. Rather, this language seems to conform to a particular pattern which is determined by the discourse community of this promotional genre. Since I aim to explore the contextual features that influence how web designers develop their webpages, the findings reveal that the variations are related to marketing strategies.

My findings regarding move analysis and register analysis are closely aligned with the notion of conventionality suggested by Bhatia (1993), who argues that texts in the same genre are likely to have same overall communicative purposes. Regardless of the nationality of the members in the community, they tend to use move structures and linguistic resources within a broad range of typical conventions. In this respect, I consider the distinction in move patterns and lexico-grammatical features between the Thai and New Zealand hotels to be minor. This is because the findings showed that the hotels in both countries use a similar move structure. Each of the two datasets has one different obligatory move but the overall communicative purposes of the hotel webpages are the same. Additionally, there is a small variation in the use of linguistic features between the two countries. The interviewees revealed that this distinction is as a result of difference in scale and marketing practices, as well as how the hotels in two countries are operated.

Since texts and images ideally work together to communicate promotional materials to the audience, the use of visual images is also an important focus of my study. I attempt to explore how web designers use images to promote hotels. The findings of visual analysis revealed that the use of 
conceptual images is more common than the use of narrative images in order to advertise the hotels in websites from both countries. Conceptual images are adopted to describe and portray atmosphere and ambience of the hotel in a way that the texts cannot. In contrast, narrative images, in which the participants are connected to one another through vectors are used to narrate the action or story that the hotels intend to convey to the web readers. Often narrative images feature people performing action. My findings extend the work of three previous studies (Francesconi, 2011; Hiippala, 2007; Suen, 2013). In these three studies, conceptual images are depicted to represent the core values of the promotional materials. For example, in Francesconi (2011), the core value of the Malta tourist brochures is heritage, which is represented through the images of megalithic temples, baroque palaces, traditional food and religious processions (p. 374). However, these previous studies do not explain the reasons why the use of conceptual images is more common than narrative images in hotel and tourism promotional text. The interviews with the Thai web designers shed light on this issue. One of the web designers suggested that one of the most important reasons why conceptual images are more common than narrative images is because without human characters, the focus of the audience is drawn to the hotels' facilities, and environment.

These studies, like my study, found greater use of conceptual than narrative images. However, a difference between their findings and mine was that the majority of boutique hotels in my dataset do not represent cultural aspects as the principal value as, for example, the visual elements in Francesconi's (2011) study do. This difference may be because of a distinction in the main focus of Francesconi's and my study. In Francesconi (2011) the visual images depicted in the tourist brochures represent the importance of cultural values, which symbolise the identity of Malta as a country. On the other hand, hotels in my dataset were private sector hotels, and their primary focus was on their marketing value. Interestingly, my data reveals that the underlying purpose of presenting these conceptual images is to highlight the idea of holiday-related experience: elegance, luxury, and relaxing or recreative state. Only three hotels (T22, T8, and T2) in the Thai dataset also depict Thai architectural design in addition to holiday related experience. Overall, my findings support Suen's (2013) visual analysis of hotel homepages in Hong Kong. Her study showed that the use of conceptual images not only represents the atmosphere or the mood (p. 183) of the hotels but also reflects the hotel's main marketing focus. On the other hand, web designers use Narrative images, where human characters are depicted, to encourage the readers to imagine themselves experiencing those services.

The use of representational meaning has no complications in my visual analysis; however, the application of Kress and van Leeuwen's (2006) idea of angle and power relationship is subjective and problematic in my study. This is because boutique hotel promotional materials have their own 
unique objectives and distinctive characteristics. As a result, Kress and van Leeuwen's (2006) interactive meaning is not wholly applicable in my dataset. In addition, the context and given examples, in which Kress and van Leeuwen (2006) explain their theory, are often limited. This is due to the fact that the examples they provide are mainly human represented participants, and it is more convincing for the readers to relate power with people. In contrast, in my study, most images are hotel buildings, suites, and facilities as well as services, where the notion of power is more problematic to assign. Unlike Kress and van Leeuwen's (2006) suggestion, the interview data with the twelve hotels confirms that the hotels do not use these three angles (high angle, eye level, low angle) to establish relationships of power with the web readers. Rather, it revealed that the use of three different angles is used to promote hotel facilities and landscape and to create atmosphere and mood in the web readers.

There is a single case in which the use of angle and power relation proposed by Kress and van Leeuwen (2006) is applicable in the hotel web advertisements. This case is found in the use of medium shot images, which is employed to establish an equal relationship between the hosts/hotel staff members and the viewers. Also, it corresponds to the underlying purpose of promotional genre of hotel online advertising, which is the goal of connecting and engaging the readers.

I investigate whether cross-cultural variations are the influential factors that determine the visual representation of Thai and New Zealand boutique hotel websites. The findings show that the depiction of the visual elements in these two countries generally does not differ. Rather, they seem to be representing an homogenous genre because they conform to the same practices. Both datasets tend to maintain their unique characteristics, where the fonts, layout, color and textual elements have to comply with the same guidelines to uphold a corporate identity. That is to say, the design of hotel webpages has its own integrity, which web designers usually follow. Web designers design websites in order to suit and satisfy readers' expectation.

The relationship between the text in a specific genre and its audience is important as web designers need to design websites to suit the expectation of their audiences. Even though the active engagement of the audience is not explicitly emphasised in English for Specific Purpose (ESP) genre studies, Paltridge (1997) argues that ESP focuses on the role of reader as much as Systemic Functional Linguistics (SFL) does. He suggests that in order to structure texts to suit a specific target group, the target group's shared understanding and expectation of the development of texts should be carefully considered. The focus on the role of the audience is also evidenced in my study in terms of textual elements that suggest the individuality of the readers (e.g. the frequent use of boutique, private, unique), and further confirmed by the interviews with hotel owners and web designers. 
My study shows the relationship between the promotional genre and its language structure and the representation of visual design. The use of move structures, distinctive linguistic features, and images associates with the core values of promotional genre which are informative and persuasive. Since the main purposes of promotional genre are to give information about the facilities and services to potential customers, and persuade them to buy the facilities and services, web designers use verbal and visual techniques to achieve these purposes. For example, the web designers use Move: Describing rooms to inform the readers about their facilities and services, and Move: Introducing the hotel/facilities/services to engage with the readers. Move: Establishing credentials is used to persuade the readers to visit the hotel by providing positive descriptions of the hotel, which includes location, history, and accomplishments of the hotel. For visual images, medium shot images are employed to build a friendly relationship with the audience. The above discussion confirms that both texts and images work together to achieve the intentions of hotel online advertising.

According to Berkenkotter and Huckin (1995) and Chandler (2000), genres change over time as they respond to users' needs, economic change, and technological factors. As a result, conventions of a particular genre shift or new genres arise. My study of hotel webpage genre is also in response to users' needs because of the introduction of online advertising in the Hotel and Tourism industry. The Internet has transformed hotel brochures into hotel webpages, where more space is provided to serve the increasing importance of images' role in web advertising.

The integration of language and visual images works complementarily to create informative and persuasive value in this promotional genre. This suggests that texts complement visual images and vice versa, and web design enhances the effectiveness of the presentation of these two elements on hotel webpages. For example, images and the use of adjectives can help to evoke the atmosphere of a hotel. The images depicted are likely to stay in the memories of the audience by playing to their imagination and emotion through the textual descriptions of the hotels atmosphere. Apart from the combination of textual and visual elements, the functionality of website, which includes the interactive part of the site, is also important to develop hotel web advertising. However, in this study, the functionality is not my main focus. I only pay attention to how web designers locate navigational links and banners. However, an investigation of characteristics and functions of the functionality of hotel webpages should be conducted in future research. The above discussion supports the idea that genres are not fixed and the purposes of the promotional genre govern how web designers use textual and visual elements to achieve their intentions. 


\subsection{Conclusion}

In this chapter, the main findings of the study have been discussed firstly indicating that genre theory proposed by Swales (1990) and Bhatia (1993) are applicable in the analysis of hotel web advertising. The findings showed that the main purposes of hotel webpages are to inform potential customers about the facilities and services as well as to build a positive image, to engage with potential customers, and to persuade them to take action. These purposes govern the rhetorical structures, linguistic features, and visual images. The findings also show the changing nature of promotional genres in response to the introduction of the Internet and the current needs in the hotel and tourism industry. 


\section{Chapter 9: CONCLUSION}

As discussed in Chapter 1, this study was inspired by my students' desire to understand the characteristics of boutique hotel webpages as potential language descriptions. This study is a genre analysis of boutique hotel webpages, focusing on an investigation of persuasive language, visual images, boutique hotel owners/web designers' perspectives, and web viewers' attitude. Bhatia's (1993) genre analysis of promotional language is the primary approach applied to explore the rhetorical structures and prevailing linguistic features of boutique hotel webpages. For my textual analysis, I focused on the identification of move structures as well as the distinctive lexicogrammatical features which are associated with each move. For the investigation of visual elements, I adopted Kress and van Leeuwen's (2006) visual analysis model to examine the characteristics and the organisation of the visual images presented on the hotel webpages including the relationship between these images and the readers. To investigate boutique hotel owners/web designers' perspectives, interviews with hotels owners, managers, and web designers were conducted. The main purpose of the interviews was to examine their perspectives and the cross-cultural values that influence the variation between the way the hotel owners and/or web designers in Thailand and those in New Zealand promote their websites. Furthermore, my study used interviews to explore the attitude of web audience toward the representation of textual and visual choices on the chosen hotel websites. The second section includes the pedagogical, professional, and methodological implications. The final part is the study's limitations. In the following sections, the major findings in relation to the research questions are summarised and discussed.

The research questions are:

1. What are the moves in English boutique hotel websites in Thailand and in New Zealand?

2. What are the distinctive lexico-grammatical features used to serve genre-specific functions?

3. What are the features of visual images in English boutique hotel websites in Thailand and in New Zealand?

4. What are the similarities and differences between boutique hotel websites in Thailand and in New Zealand?

5. What contextual factors influence the variation of the textual and image features in boutique hotel websites in Thailand and in New Zealand? 


\subsection{Major findings}

This section presents major findings of my study, which are divided into three aspects: textual analysis, visual element, and cross-cultural investigation. The analysis of the textual and visual elements reflect the main purpose of hotel advertising genre as this genre is informative and persuasive in nature. The findings of this study suggest that hotel web advertising genre requires specific rhetorical structures, genre- specific linguistic features, and unique use of visual design to serve the informative and persuasive value of the genre. The discussion of the findings respond to the research questions.

\subsubsection{Findings of the textual analysis: move analysis}

This section discusses the first research question that sought to identify the move structures in English boutique hotel websites in Thailand and in New Zealand. Even though the findings showed that there is variation between these two datasets, the variation does not impact the overall communicative purposes of the online promotional genre. This variation includes the total number of obligatory and optional moves that occurred in each dataset. Interviews with the hotel owners/managers in both countries, and web designers in Thailand, suggested that these variations may be caused by the 1) differences in cultural background and social contexts between Thailand and New Zealand, and 2) the distinction in the way the boutique hotels are established and operated between these two countries.

Since genres are primarily recognised by the purposes that they serve, the frequency of the occurrence of moves reflects the main communicative purposes of the genre. This analysis indicates that boutique hotels in these two countries have a different focus when advertising their hotels. In the Thai dataset, ten moves were identified, with four of those being obligatory. They include 1) Introducing the hotel/facilities/services, 2) Establishing credentials, 3) Describing the offers, 4) and Describing the rooms. These four moves are the main communicative purposes of the boutique hotel in the Thai dataset. On the other hand, in the New Zealand dataset, eleven moves were found, with four obligatory. They are 1) Introducing the hotel/facilities/services, 2) Establishing credentials, 3) Describing rooms, and 4) Calling for action (see Table 7 in Chapter 5). The occurrence of these obligatory moves reflects the way the hotels in both datasets position and promote themselves as boutique hotels.

In spite of the fact that these Thai and the New Zealand websites are recognised as the same genre containing a similar set of primary communicative purposes of promoting boutique hotels and persuading the readers to make a reservation, they differ in several aspects. The first difference is in 
the way the information is organised and structured in each dataset. In order to explore the factors behind the derivation of conventions and variation in the analysis of boutique hotel webpage genre, I interviewed six Thai hotel owners, four Thai hotel managers, four Thai web designers, and five New Zealand hotel owners. Possible explanations for the subtle variation between the two datasets are, firstly, the difference in scale of operation between the hotels in the two countries. The second reason is that the process and practice of producing webpages is somewhat different between the web designers in each country. A difference was revealed by the interview with the Thai hotel's manager. He explained that he hired a professional copy writer to develop promotional texts for the hotel.

Despite the variation between these two datasets in terms of number and types of moves including the way the hotels in Thailand and New Zealand are established and operated, both datasets share three similar obligatory moves, which are the main purposes of this hotel web genre. They are Move: Introducing the hotel/facilities/services, Move: Establishing credentials, and Move: Describing the rooms. This means, hotel web designers in the two countries consider that the three obligatory moves are the most common way of promoting the hotel and communicating with the web audience. Since the brand name of the hotel is an important symbol indicating the type of hotel, web designers aim to use Move: Introducing the hotel/facilities/services to introduce the name of hotel, but at the same time web designers intend to differentiate their hotel from the other types of hotels (i.e. chain hotel, guest house, and bed and breakfast). This is evidenced by the frequent use of boutique in combination with the name of the hotel in my datasets. It seems to be one of the useful marketing strategies to distinguish their hotel from other kinds. Another purpose of this move is to greet and welcome the audience. The next move that web designers believe is important in order to advertise their hotel is Move: Establishing credentials. The function of this move is to convince the potential guests to make a decision to book the hotels by persuading them to believe that the hotel is well-established with outstanding and unique services and facilities. The last move is Move: Describing the rooms. The web designers use this move to describe the rooms and amenities provided by the hotel. The descriptions include the sizes, facilities in the room, and decorations. In order to persuade the audience to visit the hotel, the web designers use the three obligatory moves to achieve the informative and persuasive value. In addition, the findings on the function of each move enable novice web designers to understand how each purpose is realised and achieved. In essence, move analysis suggests that the web designers from both datasets similarly use the three obligatory move structures to develop hotel advertising text in order to inform potential customers about the facilities and services, to build a positive image, to engage with potential 
customers, and to persuade them to take action. The knowledge of rhetorical moves and their functions can be used as basic guidelines for novice web designers in hotel webpages development.

\subsubsection{Major findings in textual element: Register analysis}

Apart from the analysis of move structures, the identification of prominent linguistic features is also essential in genre analysis. Register analysis describes functions and collocational patterns of the distinctive linguistic features in the present study. The functions and the patterns of the common features help novice writers to understand how each move is structured linguistically by experts in the discourse community. As a result, novice writers can choose proper linguistic features to achieve the function of each move and then to fulfil the overall purpose of the promotional genre. At a register level, the use of these three features suggests that the hotel webpage genre are informative and persuasive.

The following section reveals the overall distinctive persuasive devices that the hotels use to achieve the main purposes of this genre. In this study, the analysis of distinctive linguistic features was conducted only for the five obligatory moves and one optional move - Introducing restaurants/ bars, and lounges - that have a high frequency of occurrence in both datasets. The linguistic analysis covers three main types of prominent features: 1) verbs, 2) adjectives, and 3) pronouns. I also investigate whether the cultural variation between these two countries influences the way web designers adopt promotional language to advertise their hotels online.

\section{Verbs}

This section discusses the differences and similarities of how the web designers use the most distinctive verbs to promote the hotels and interact with the audience between the Thai and New Zealand datasets (see table 45). The similarities are found in three moves - Introducing the hotel/facilities/services, Establishing credentials, and Describing rooms. The hotels in both datasets adopt a similar set of verbs: welcome/invite, verb to have, and offer. These four verbs are the common features that are used to achieve the persuasive value in online advertising texts. The web designers use welcome/invite in order to interact with the readers. For verb to have, it has a specific function in Move: Establishing credentials. Verb to have is used to introduce facilities and service, to inform local cultural knowledge, as well as to describe background and history of the hotels. In order to advertise the accommodation, web designers use the verb offer to describe the types and designs of the room. These verbs are a potential useful linguistic resource for novice web designers. It is important for novice web designers to learn distinctive words and understand their specific functions and patterns in order to use them to engage with the audience. 
The similarities in the use of distinctive verbs across the two datasets were clarified by the interviews with the hotel owners/managers, and web designers. The hotel owners in New Zealand explained that they use their own intuition and feeling when writing hotel promotional texts. Similar to the Thai hotels, some New Zealand hotel owners reported that they hired a professional web designer to write their website. Also, interviews reported that many hotel owners and web designers consult other hotel websites when writing their websites. This fact may influence the way the hotels use language to convey their purposes. On the other hand, the use of the verbs in the other two optional moves - Calling for action (Thai hotels) and Describing offer (NZ hotels) - contain variations in the way the most frequent verbs are exploited to realise the main purpose. However, these variations between these verbs, such as in, the verbs contact and book, still similarly maintain the main communicative purpose. Examples include "Contact our e-concierge for details" (T12) and "Book now" (NZ6). The hotel web designers use these two sentences to encourage the readers to take action.

Table 43: The use of verbs in the Thai and New Zealand datasets

\begin{tabular}{|l|c|c|}
\hline \multicolumn{1}{|c|}{ Moves } & Thai hotels & NZ hotels \\
\hline Introducing the hotelfacilities/services & welcome, invite & welcome, invite \\
\hline Establishing credentials & Verb to have & Verb to have \\
\hline Describing rooms & offer & offer \\
\hline Calling for action & contact & book \\
\hline Describing offer & allow & relax \\
\hline
\end{tabular}

\section{Adjectives}

At a register level, the abundance of favourable adjectives suggests persuasive value of the hotel webpage genre. The web designers employ adjectives, especially positive adjectives to modify facilities and services in order to show that their services are desirable and unique. For the adjectives (see table 46), boutique and special are the two adjectives that the hotels in the Thai and New Zealand dataset share. Boutique occurred the most frequently in the two obligatory Moves: Introducing the hotel/facilities/services and Establishing credentials. The hotels use this adjective to promote themselves as a boutique hotel. It is likely to be a specific terminology that hotels use to position themselves as a chic or hip hotel (Anhar, 2001; Aggett, 2007; Covelli, 2005; Hartesvelt, 2006; Lim and Endean, 2008; McIntosh \& Siggs, 2005; Rogerson, 2010). (See more detail of these studies in Chapter 2). As discussed before in section 9.1.1, the function of boutique in the collocational pattern 
corresponds to one of the steps of Move: (I), which is step ns: Stating the name/or slogan of the hotels. The findings show that web designers use boutique to amplify the unique characteristics of being a boutique hotel as a major selling point to compete with other types of hotels. Another adjective is special. Its function is to promote about special deals to potential guests and then encourage them to respond immediately.

For the other two moves, the use of adjectives across (private and free) the Thai and New Zealand hotel webpages revealed certain differences. Owing to the difference in the frequent occurrence of private between both countries, which represents a different degree of importance of privacy that each country aims to promote to the readers, it seems that the Thai hotel websites consider privacy of the guests as the most important. On the other hand, free is the most frequent adjective in the New Zealand boutique hotels. According to Perini (2009), free conveys money saving, which is an important value in promotional genres. The frequent use of free in my study supports Perini's (2009) study. The dominant collocate behaviour of free is associated with Wi-Fi. It seems to be the most important feature that the New Zealand hotels aim to promote, which can be seen from the high co-occurrence of free and Wi-Fi. Consequently, boutique hotels in New Zealand aim to promote free $\mathrm{Wi}-\mathrm{Fi}$ as a prestigious feature to satisfy their target customers who may be business travelers who have to keep in contact with their business partners. The findings of register analysis underlines the importance of domain-specific adjectives such as boutique that are commonly used in boutique hotel webpages. This is because the use of adjectives can portray and characterise the types of hotels as well as the services and facilities provided. The selection of positive adjectives highlights the core value of the hotel advertising genre, which are informative as well as persuasive, and important to the construction of hotel advertising texts.

Table 44: The use of adjectives in in the Thai and New Zealand datasets

\begin{tabular}{|l|c|c|}
\hline \multicolumn{1}{|c|}{ Moves } & Thai hotels & NZ hotels \\
\hline Introducing the hotelfacilities/services & boutique & boutique \\
\hline Establishing credentials & boutique & boutique \\
\hline Describing rooms & private & free \\
\hline Calling for action & special & special \\
\hline Describing offer & Thai & available \\
\hline
\end{tabular}




\section{Pronouns}

Interestingly, the hotels in the Thai and New Zealand datasets use a similar set of pronouns, which includes our and your (see table 47). The similarity in the use of pronouns between the two datasets implies that our and your are common pronouns in hotel web advertising texts. The main purpose of the use of these pronouns is to engage readers with the hotel promotional texts because hotel web advertisement is considered as an interaction between two parties: hotels and target guests. As a result, the hotels aim to focus on the audience's benefit and interest rather than other people.

In my study, our refers to the hotel when they are promoting and describing their services as well as the facilities. Your refers to target audience. The pronoun your refers to ownership of you as a target guest. The primary intention of using your by the hotels is to make the readers feel included and that their individual desires are recognised. Very often the occurrence of your in my dataset is used to create privacy and personal space that the guests will have when they visit the hotel. The pronoun our is used as a reference to the ownership of the hotel properties as well as their facilities and services. The interviews with the hotel hosts in New Zealand supports my register analysis that the frequent use of pronouns our and your in hotel webpages is one of the common persuasive strategies that is used to address the audience and directly engage their attention.

Table 45: The use of pronouns in the Thai and New Zealand datasets

\begin{tabular}{|l|c|c|}
\hline \multicolumn{1}{|c|}{ Moves } & Thai hotels & NZ hotels \\
\hline Introducing the hotelfacilities/services & our & our \\
\hline Establishing credentials & you & we \\
\hline Describing rooms & your & your \\
\hline Calling for action & our & our \\
\hline Describing offer & your & your \\
\hline
\end{tabular}

\subsubsection{Major findings in visual image analysis}

This section discusses the third and the fourth research questions that sought to investigate the cross-cultural variation of boutique hotel online advertising by considering the similarities and differences between the semiotic representation of Thai and New Zealand boutique hotel websites. The visual analysis is based on three metafunctions proposed by Kress and van Leeuwen (2006): the visual roles of images (representational meaning), the relationship between represented participants 
and viewers (interactive meaning), and the layout presented on the webpages (compositional meaning). The findings reveal some distinctive patterns through the use of these three meanings.

From the perspective of the visual roles of images (representational meaning) proposed by Kress and van Leeuwen (2006), the Suites page is the most distinctive page because it contains the largest number of images across two datasets: 49 images (32\%) in the Thai dataset and 41 images (31\%) in the New Zealand dataset. Interviews with the two web designers in the Thai dataset suggested that the hotel owners believe that accommodation is the most important selling point for attracting their main source of income from the potential guests. As a result, hotels present the information and images of types of room, design and decoration of the rooms including the details of the facilities and amenities provided in each room. That is to say, the hotel owners use the Suite page to encourage the viewers to make a booking.

With regard to the representational meaning, the types of images depicted, whether they are conceptual or narrative images, reflect the different degrees of the relationship between the viewers and the images envisaged by the web designers. Conceptual images are employed to describe atmosphere and environment of the hotel. In contrast, the hotels use narrative images to narrate the story to the audience. The findings show that the use of conceptual images is more common than the use of narrative images in order to promote the hotels from both countries. Narrative pictures are often used to depict a story where there are human represented participants (RPs) as main character. Common examples of the depiction of narrative images include spa and function service, where human RPs are performing certain activities or using hotel facilities and services.

The interviews revealed why narrative images where the human participants are the main characters are less common than conceptual images in promoting boutique hotel websites. The first reason is that the hotels have to spend more money to hire models. In addition, human RPs images are mainly adopted in specific contexts, such as Spa, Restaurant, and Function pages. As a result, only large-scale hotels where spa and function facilities and services are included use human participant images to promote these two pages. Smaller scale hotels where spas and special functions are not provided rarely use this kind of image. The second reason is sometimes the use of narrative images distracts the audience's attention from hotel's building and environment to human characters

In short, in regard to the representational meaning, it is more common to use conceptual images to promote hotels' architecture and environment. My visual analysis shows that the cultural differences between the two countries in the depiction of conceptual and narrative images do not 
determine the way the hotels use images to promote their hotels on their webpages. This is because the overall marketing approaches in terms of their use of visual images of the hotels in these two countries do not differ greatly. Based on these findings, it seems that the hotels in both countries use similar promotional strategies in advertising their hotels through visual representation.

The next aspect of visual image analysis is the relationship between represented participants and viewers (interactive meaning). The interactive meaning involves how the images and the viewers interact in order to create an imaginary relationship. The hotels prefer using offer image to depict hotel buildings, surrounding environment, and facilities as well as services. Also, the demand image is often used to depict hotel hosts and hotel staff members in order to greet and establish friendly relationships with the readers. In addition, there is no distinction as to how the hotels in the Thai and New Zealand datasets use demand and offer images to promote their hotels.

Regarding the idea of angle and power adopted in the boutique hotel websites in Thailand and New Zealand, a medium shot images are the most frequent used. However, in most cases, the use of a medium angle in my dataset does not connote any type of power relation or represent an equality between the RPs depicted in the images and the viewers. Rather, a medium shot is often used to represent hotel architecture and suites. At this angle, all the details of the design and facilities in the hotels and rooms are highlighted and are easy to observe by the viewers. Images taken from the low angle is not common in both datasets because the hotels avoid exercising power over viewers or showing that the hotels are looking down on the audience.

In both datasets, the depiction of an oblique angle and long shot images do not indicate detachment. These types of images are often used to promote hotels' exteriors, suites and restaurants. On the other hand, a frontal angle is generally applied to picture restaurants in the Thai dataset and food in the New Zealand dataset.

In regard to interactive meaning, my study shows that the relationship between the hotels and the viewers cannot be fully characterised through the use of angle and the distance between the images and the viewers. The interpersonal relationship between the images and the viewers also depends on what kinds of represented participants are presented in the images and the main intention of the hotel that they aim to convey. Interviews with hotel owners and web designers in Thailand and New Zealand confirmed that the use of camera angles does not symbolise the degree of intimacy between the images and the viewers in boutique hotel contexts. Instead, the hotels use images depicted with different angles to promote hotel facilities, accommodation and landscape, and to create atmosphere and mood of the web readers. 
For the compositional meaning, it is more common for the hotels in Thailand and New Zealand to use ideal-real distinction to depict contrasting elements in the upper and lower parts of the webpages. Cultural differences between the two countries do not influence the way the hotels present their information and images on their webpages. Name, logo, and navigational links are usually situated on the top or ideal section. Beneath the navigation links are where the main welcoming images (banners) depicted in the display banner located. In contrast, the bottom or real section of the webpages provides the adress, contact information of the hotel, and information about web designing company.

For given-new structure; however, it is more typical for the New Zealand hotels compared with the Thai hotels. The hotels position their photographs as given elements on the left and their texts as new elements on the right. The hotels highlight the fact that visual images that are located on the left are familiar elements that guests have viewed and texts on the right are the offers that the hotel promises to give to the guests when they book the hotel. Interviews with the Thai web designers confirmed the given-new approach seems to be obsolete in the Thai context. The distinction in the use of web layout between the Thai and New Zealand dataset signifies the differences in the value of web design and the nature of boutique hotel business in these two countries.

To answer the research concerning the feature of visual images depicted on hotel webpages, the visual analysis suggests some typical feautues of visual images across the two datasets. The application of the three metafunctions showed that the web designer aim to convey informative and ppersuasive messages through the use of conceptual images, medium shot images, and ideal-real construction.

\subsection{Implications of my study}

This study has its roots in my experience as a tourism and hotel business teacher, which revealed a need for thorough descriptions of generic structures of hotel and tourism materials. I found that my students required a language description of the move structures and lexciogrammatical choices in the materials that could benefit them outside the classroom. The students required language guidelines that they could use in real life after they finished the course.

Consequently, I have applied genre analysis to Thai and New Zealand boutique hotel webpages in order to develop materials on language descriptions of webpages for hotel and tourism courses. My study is the result of discovering my students' needs in language descriptions. This study aims to make a contribution to materials for second language learners who take courses in ESP (English for 
Specific Purposes) and novice web designers or hotel owners who would like to develop their own boutique hotel website.

Apart from pedagogical contributions to ESP teachers and students, which I enlarge on in section 9.2.1 below, my findings with regard to the textual elements and web design construction also provide resources for the knowledge and practice of novice web designers and hotel owners. They can use my language descriptions and web designs guidelines to construct and design their websites without spending money and consulting professional web designers and copywriters. I discuss this further in section 9.2.1

This study is a genre analysis that investigates the specific discourse of boutique hotel webpages. The main purpose of the boutique hotel websites was found to be clearly persuasive. This finding was supported by the occurrence of all the move structures, distinctive lexico-grammatical features, and the presentation of the visual images in both the Thai and New Zealand datasets. The analytical approach chosen for textual elements was genre analysis in the tradition of English for Specific Purposes (ESP) proposed by Swales (1990) and Bhatia (1993).

The main purpose of ESP genre analysis is to identify move structures that reflect the communicative purposes in texts and then analyse the typical lexico-grammatical realisation of these moves. For visual analysis, the framework of Kress and van Leeuwen in Systemic Functional Linguistics (SFL) was adopted to explore the relationship between the visual elements and the web audience. However, genre knowledge requires more than just knowledge of textual structures and linguistic features. It also requires an understanding of the professional context where the genre is situated. This is because according to Bazerman (1997, p. 19), genres are not forms but rather frames for social action, where meaning is constructed. Bazerman's (1997) suggestion is similar to Hyland's (2003) perspective of the application of genre in teaching writing, in which Hyland views the courses as more than just an abstract activity but social practices. In this respect, the interviews with the hotel owners and web designers were conducted in order to understand the contexts in which the genre is situated. My interview data enhanced the understanding of the differences and similarities between the characteristics of the boutique hotels in the Thailand and New Zealand contexts. Second language learners, novice web designers, and boutique hotel owners can benefit from the identification of the recognisable structural identity and generic integrity of a particular genre. With the provision of generic integrity and contextual knowledge of where genre is situated, these group of people can have clear and specific guidelines to the structure and purpose of boutique hotel webpages. 


\subsubsection{Pedagogical implications}

The primary purpose of genre-based pedagogies is to teach learners how to use language structures to achieve their communicative purposes. Applying Swales's (1990) influential genre analysis approach, in which his fundamental objective is to help non-native learners of English read and write research article introductions, my study offers a resource for writing of promotional materials that I hope is not only limited to the domain of boutique hotel online advertising but also to show how genre analysis can benefit ESP teaching and learning especially in hotel and tourism courses.

Even though genre-based pedagogies are the result of genre analysis, as a teacher, it is important to let the students know that these patterns or conventionalities are not static. This is because the organisational patterns and linguistic features depend on the situational contexts where the genre is located and the communicative purposes that the writers aim to convey. As a result, the language structures that are derived from genre analysis can be used as a writing foundation but not a prescriptive blueprint to which students have to strictly conform. For teachers, it is important to be aware of the English proficiency level of the students in order to appropriately design teaching lessons to suit their learning level and style. This is particularly so, in the Thai educational context, where Thai students perform a passive role in a classroom. According to Khunnawat (2009), Thai students prefer a clear and explicit teaching style. They tend to follow instructions and avoid contradicting teachers. In such learning environments genre-based teaching approaches may be more readily accepted. However, it can also lead to failures in acquiring language structures. This is because the explicit provision of rules and grammar can lead to overgeneralisation of rules by students (Freedman, 1993) and prevent them from having the freedom and creativity to develop their writing skills. (Feez, 2002; Francesconi, 2012; Kay \& Dudley-Evan, 1998). As a result, in a genrebased classroom, it is necessary to raise the students' awareness of the importance of social conventions and situational contexts when constructing their messages to the readers. Instead of mainly focusing on forms and grammar, it is important for teachers to provide systematic scaffolding and emphasise the relationship between social constraints and relevance of lexico-grammatical choices. Incorporation of textual patterns and their situational contexts into genre teaching can enhance students' learning experience and success in writing.

In addition to the move structures, my findings regarding the register analysis of boutique hotel webpages offer a valuable descriptionof specialised tourism language, which can be used to develop lessons promoting skill in the writing of promotional language for learners. It is important to prepare students to gradually recognise the unique characteristics of the promotional language that 
are typically found in the boutique hotel industry. Teachers should encourage students to pay special attention to those unique properties in order to understand and be able to reproduce them in appropriate contexts. At a later stage, with the proper and systematic support from teachers, students should be able to transfer these acquired promotional features and create their own promotional materials in broader contexts. However, this practical transference of acquired promotional language skill may be expected only in advanced language learners who are able to invent creative ways of writing promotional language for hotel and tourism contexts. An example of genre-based teaching materials are presented in appendix 7.

\subsubsection{Professional Implications}

Even though this study was limited to three main webpages (Home, Suite, Facility) of boutique hotels and the findings are based on the result of genre analysis, the results of the study can serve as a foundation for writing promotional materials in English especially for hotel practitioners and novice web designers. The importance of this study is that it provides information about the main communicative purposes, distinctive lexico-grammatical features, visual images and the possible influences of cultural differences in the way boutique hotel webpages are designed and structured as a genre.

Due to the fact that tourism and leisure products are intangible, inseparable, variable, and perishable (Morgan \& Pritchard 2000), the effective use of hotel and tourism advertising is mainly based on the construction of the readers' imagination through promotional messages. Successful promotional advertising materials can only be produced with the effective use of the visual design and textual elements. With the introduction of the Internet in the hotel and tourism sector, hotel facilities and services can be more effectively presented than through any other existing media. This is because a better website quality can influence the booking decisions of potential guests. Thus, it is essential for professionals in the hotel and tourism domain to be aware of the presentation of content, the use of visual images, and medium required to enhance the persuasive effect on readers

I hope the findings of my study can contribute to the knowledge and the practice of hotel owners/practitioners, novice web designers, and copywriters who aim to promote their hotel webpages in English. Importantly, my analysis of move structure and the linguistic features in the boutique hotel websites that are used to realise the purposes of the individual moves (move register) can be used as basic textual guidelines for novice web designers to develop their own webpages to suit their hotel contexts. Not only does this study provide language descriptions, but it also offers information on how to design the webpages based on my findings and interviews with the hotel owners and web designers. 


\subsubsection{Methodological Implications}

This section presents two methodological implications of my thesis. Building on Kress and van Leeuwen's (2006) visual image framework, my study shows the remarkable consistency and regularity of the representational, interactive, and compositional meaning of the images. Yet, my findings also show that this visual image analytical framework is only partially applicable to my dataset. The most problematic aspect lies on the interactive meaning; more specifically the use of angles to convey the relationship between the images and the readers. According to Jewitt and Oyama (2001, p. 145), interactive meaning refers to imaginary relations between images and viewers of images that occur through their interaction. Even though the framework of Kress and van Leeuwen (2006) offers a practical description and suggestions to examine images in a variety of contexts, it contains certain drawbacks when dealing with images in the hotel and tourism field. This is because the interpretation of the meaning and the relationships between the images and the readers varies according to the context of the situation. The explanations and examples given in the framework are based on magazine advertising, where the main characters are human beings. In addition, the description and criteria of how angles establish different kinds of relationship and power is applicable where the depicted images are human beings rather than buildings and landscapes. This is where the issue of subjectivity of this framework began in my analysis because my dataset relies heavily on architecture, hotel buildings, hotel suites, and landscapes. In fact, the interpretation of this framework is not satisfactory when dealing with architecture and landscapes. These emerging problematic issues were confirmed and supported by my findings and the interviews with the different hotel owners including the web designers. My study revealed that the use of oblique images, which Kress and van Leeuwen (2006) and Unsworth (2001) view as conveying a lack of involvement between the images and the viewers, is not relevant to the main intention and the persuasive value of the hotel promotional texts.

Instead of denoting a sense of detachment, the oblique pictures in my dataset are actually being used to promote the design, layout, extent or surroundings of hotel buildings, suites and restaurants, all of which assign meaning and value to the non-human images as I discussed in chapter 7: Visual Analysis. The interview data with the owners of the hotels also clarified the objective of presenting oblique images. The first reason is that, since the hotels generally have different suite layouts and configurations of their floor plans, the oblique angle is more accurate to use to capture the actual layout of the suites. The second reason is that the use of an oblique angle allowed the hotels more flexibility to capture the suites and the attractive part of the hotels' exteriors against the beautiful natural background than using a frontal angle. 
Within the interactive meaning, apart from the incongruity of the inherent assigned value of oblique angle images and its applicability in a promotional context, a second problematic aspect of the framework is the meaning it assigns to the use of high angle and low angles. Kress and van Leeuwen (2006) proposed that these two angles establish different levels of pseudo power between the images and the audience. This relationship between power and the angles is not applicable in boutique hotel promotional materials because they have their own unique objectives and distinctive characteristics. Instead of representing power relations between the images and the potential guests, the purpose of the use of high and low angles in my dataset is to show different angles of the hotel building and surrounding landscape as well as to create atmosphere and mood for the web readers.

With regard to the methodological framework, fundamentally, my findings contradict the interactive meaning proposed by Kress and van Leeuwen (2006) since their descriptive criteria and purposes of the framework are not applicable to my dataset. Although Kress and van Leeuwen's framework contains a major drawback when applying it to the hotel and tourism context, similar studies (Suen, 2013, Francesconi, 2011) have adopted this framework to analyse their images in this genre without addressing this issue. To solve this problem, this framework should be modified and extended to include images which are largely not narrative images, and where issues of power are less likely to be salient. For example, in the tourism and hotel area, oblique angles are mainly used to capture different perspectives of suites and restaurants to gain accurate points of view of the suites. Meanwhile, high and low angles are used to depict the different perspective of the design of the hotel buildings, and landscape in order to create a persuasive value in the hotel advertising materials. Researchers who aim to adopt this framework in the promotional material should be aware of these issues and allow themselves flexibility to adjust the value as well as the criteria to suit a specific intention and the characteristics of the dataset.

Due to the fact that ethnographic methods have not been widely used in promotional genre studies, this section discusses the contribution of the integration of interviews to genre analysis. In my study, this approach was used to explore the perspective of the expert members in boutique hotel webpages: web designers, hotel owners, and web audience. Since a major shortcoming of genre analysis in the ESP tradition is that there is a degree of subjectivity in move identification (Dudley-Evans, 1994; Kanoksilapatham, 2003; Paltridge, 1994), Bhatia (2004) and Swales (1990) suggest that insightful information from the experts in the discourse community will enhance the clarity of the moves. Bhatia (2004) points out that identifying genre requires more than linguistic feature and discoursal patterns. In fact, it requires knowledge of the contexts in which the genre is 
situated and information about the behaviour and relationships of the members of the discourse community.

Despite the fact that many genre analysts are aware that they need an ethnographic approach to validate their findings about the move demarcation and linguistic features, only a few genre studies have combined these approaches (Suen, 2013; Flowerdew and Wan, 2006). In my study, the experts in the genre community are hotel owners, hotel web designers, and the web audience who are the potential customers of the boutique hotel context. The web designers and hotel owners' points of view help clarify the reasons behind their writing and the design of the webpages. Also, the interviews minimise this subjective nature in genre analysis and help to triangulate the findings from the textual and visual analyses.

This study is a genre analysis of boutique hotel webpages, which provided insights into the knowledge about rhetorical structures, lexico-grammatical features, and web design. As more people are depending on the Internet to look for hotels, the findings show that the language and web design used to promote hotels in Thailand and New Zealand tend to follow genre integrities to target their potential visitors.

It is usually believed that different cultures may influence different expectations in terms of linguistic choices that the authors are wired to produce in texts (Hyland, 2000). However, the question of whether cultural elements affect the choice of linguistics features has been a controversial issue among genre theorists. Crossley (2007) proposes that moves are cultural. In his view, when a writer travels between cultures but remains within a similar genre, the move expectation of that genre may change. On the other hand, Bhatia (1993) argues that texts in the same genre are likely to have the same overall communicative purposes whatever culture the writers are from, since they tend to use move structures and linguistic resources within a range of typical conventions to address to very similar audience.

In this respect, I am aware of the possible influence of cultural background that exits where the genre is situated when analysing genre in my dataset. However, my findings show that there are only minor differences in terms of move structures and linguistic features that hotel professionals have to be aware of when developing hotel advertising online. The role of culture has more influence on the differences on webpage design and layout between Thai and New Zealand hotels. These variations are caused by the fact that the scale of the boutique hotels in Thailand is larger than the hotels in New Zealand; the Thai hotel texts focus more on promoting their services and facilities to attract the readers' attention. Conversely, the New Zealand hotels highlight the benefit of personalised services and focus on building relationships with the guests. This minor variation shows the flexibility of how to design hotel promotional texts. Since the hotel advertising texts are 
dynamic and contain innovative and unique features of lexical choices, novice web designers should take these unique features and their hotel contexts into consideration when designing their webpages.

\subsection{Limitations of the study}

The limitations of my study are of two kinds. The first one is dataset related. The dataset used in this study was restricted to 50 boutique hotel websites for move and visual analysis, and 100 websites for register analysis. Therefore, the findings may not represent the rhetorical and visual structures of web advertising for all boutique hotels in Thailand and New Zealand. Also, this study focused on a small number of linguistic features: imperatives, passive voices, verbs, adjectives, and personal pronouns. Other kinds of features such as noun phrases and verb tenses would have been useful to explore in order to improve genre writing knowledge. However, investigation of these features were beyond the scope of this study. Next, since this study showed that local culture does not have a major role in boutique hotel web advertising in Thai and New Zealand, intertextuality could have been investigated, as interviews with hotel owners and web designers reported that they consulted other websites when developing their hotel website. This may influence the similarities in the presentation of advertising language.

The other kind limitations is related to interviews. Firstly, this study would have offered more comprehensive insights and the questions related to textual elements would have likely been better answered, if I had had the chance to interview a larger number of hotel practitioners, web designers, and copywriters. Next, before the interview phase, I thought my questions would cover all the aspects that I would like to know from the hoteliers, and web designers. However, while writing up my discussion chapter, a number of questions arose that could not be answered by hotel webpage analysis alone. Thirdly, the responses of the hotel owners about how they constructed their promotional texts were not as insightful as had been hoped. It appears that they were not aware of how they did it. The last limitation involves the complications in the web audience interviews. The participants recruited (web audience) lacked the intention of booking a hotel since I had no permission from the hotels to interview their guests. In addition, some of the participants did not have experience in staying in a boutique hotel. As a result, the information received from the interviews with the web audience was not helpful in answering my research questions. The interviews with the web audience in promotional genre studies should be further extended in order to help professional and novice web designers better understand the web audience's perspective so that they can advertise their hotels more effectively. 


\subsection{Closing remarks}

My study describes the characteristics of boutique hotel webpages as a genre. The study includes four main components which are important to structure and enhance the understanding of this genre. They include move/step analysis, register analysis, visual image design, and interviews. The findings of the study indicate that hotel web advertising genre has its own structures. The structure relies on the communicative purposes and then the purposes determine the language used and the representation of images. The conventions of the hotel webpage genre, which is the core value of genre theory are more influential in governing the characteristics of language used and the presentation of images than cultural differences between Thai and New Zealand. Since genres are not static according to several factors such as users' needs, economic role, and new technology, hotel webpage genre has emerged in response to the Internet and web users' needs. 


\section{References}

Aaron, J. E., \& McArthur, M. (1998). The Little, Brown Compact Handbook. New York: Longman.

Adam, C., \& Artemeva, N. (2002). Writing instruction in English for academic purposes (EAP) classes: Introducing second language learners to the academic community. Genre in the classroom: Multiple perspectives (179-196). London: Lawrence Erlbaum Associates.

Aggett, M. (2007). What has influenced growth in the UK's boutique hotel sector? International Journal of Contemporary Hospitality Management, 19(2), 169-177.

Aikhenvald, A. Y. (2010). Imperatives and commands. Oxford: Oxford University Press.

Almeida, D. (2009). Where have all the children gone? A visual semiotic account of advertisements for fashion dolls. Visual Communication, 8(4), 481-501.

Akkhakraisri, P. (2004). A Corpus-Based Approach to the Genre Analysis of Marketing Copy Posted on the Hotel Chain Websites. Unpublished master's thesis, Kasetsart University, Bangkok, Thailand.

Anhar, L. (2001). The definition of boutique hotels. Hospitalitynet. org. Retrieved 20 April, 2015 from http://www.hospitalitynet.org/news/4010409.html

Askehave, I., \& Nielsen, A. E. (2005). What are the characteristics of digital genres? - Genre theory from a multi-modal perspective. Paper presented at the System Sciences, 2005. HICSS'05. Proceedings of the 38th Annual Hawaii International Conference on System Sciences.

Askehave, I., \& Swales, J. (2001). Genre identification and communicative purpose: a problem and a possible solution. Applied Linguistics, 22(2), 195-212.

Badger, R. (2003). Legal and general: towards a genre analysis of newspaper law reports. English for Specific Purposes, 22(3), 249-263.

Badger, R., \& White, G. (2000). A process genre approach to teaching writing. ELT Journal, 54(2), 153-160.

Bakhtin, M. (1984). Characteristics of genre and plot composition in Dostoevsky's works. Problems of Dostoevsky's poetics, 101-180.

Bakhtin, M. M. (1986). Speech genres and other late essays. Texas: University of Texas Press.

Baloglu, S., \& Pekcan, Y. A. (2006). The website design and Internet site marketing practices of upscale and luxury hotels in Turkey. Tourism Management, 27(1), 171-176.

Barsky, J., \& Waite, M. (n.d.). Points, Price, Promotions? How Guests Select Hotels Around the World. Retrieved 28 April, 2016, from http://hotelexecutive.com/business review/3381/pointsprice-promotions-how-guests-select-hotels-around-the-world 
Bateman, J. A. (2008). Multimodality and genre: A foundation for the systematic analysis of multimodal documents. Basingstoke: Palgrave Macmillan.

Bawarshi, A. S., \& Reiff, M. J. (2010). Genre: An introduction to history, theory, research, and pedagogy. West Lafayette, IN: Parlor Press.

Bazerman, C. (1997). The life of genre, the life in the classroom. In W. Bishop \& H. Ostrom (Eds), Genre and writing: Issues, arguments, alternatives (pp. 19-26): Portsmouth, NH: Boynton/Cook.

Bell, P., \& Milic, M. (2002). Goffman's Gender Advertisements revisited: Combining content analysis with semiotic analysis. Visual Communication, 1(2), 203-222.

Berkenkotter, C., \& Huckin, T. (1995). Genre knowledge in disciplinary communities. Hillsdale, NJ: Lawrence Earlbaum.

Bhatia, V. K. (1993). Analysing genre: Language use in professional settings. London: Longman.

Bhatia, V. K. (1996). Nativization of job applications in South Asia. In R. Baumgardner (Ed.), South Asian English: Structure, use and users (pp. 158-173). Urbana: University of Illinois Press.

Bhatia, V. K. (2004). Worlds of written discourse: A genre-based view. London: Continuum.

Bhatia, V. (2005). Generic patterns in promotional discourse. In T. Virtanen \& H. Halmari (Eds.), Persuasion across genres: A linguistic approach (pp. 213-225). Amsterdam: John Benjamins.

Biber, D., Connor, U., \& Upton, T. A. (2007). Discourse on the move: Using corpus analysis to describe discourse structure. Amsterdam: John Benjamins.

Biber, D., Conrad, S., \& Reppen, R. (1998). Corpus linguistics: Investigating language structure and use. Cambridge: Cambridge University Press.

Biber, D., Johansson, S., Leech, G., Conrad, S., Finegan, E., \& Quirk, R. (1999). Longman grammar of spoken and written English. London: Longman.

Bill, P. (2001). Content analysis of visual images. In T. Van Leeuwen \& C. Jewitt (Eds.), Handbook of visual analysis (pp. 10-34). London: Sage.

Bohner, G. (2001). Writing about rape: Use of the passive voice and other distancing text features as an expression of perceived responsibility of the victim. British Journal of Social Psychology, 40(4), 515-529.

Bonyadi, A. (2012). Genre analysis of media texts. Procedia-Social and Behavioral Sciences, 66, 86-96.

Boonchayaanant, V. (2003). A genre-based analysis of tourist leaflets produced and distributed in the United States of America. Unpublished master's thesis, Kasetsart University, Bangkok, Thailand. 
Bowker, L. (2002). Working with specialized language: a practical guide to using corpora. London: Routledge.

Brown, P., \& Levinson, S. C. (1987). Politeness: Some universals in language usage. Cambridge: Cambridge University Press.

Brown, R., \& Gilman, A. (1960). The pronouns of power and solidarity. In T. A. Seboek (Ed.), Style in Language (pp. 253-276). Michigan: MIT Press.

Bull, P., \& Fetzer, A. (2006). Who are we and who are you? The strategic use of forms of address in political interviews. Text \& Talk - An Interdisciplinary Journal of Language, Discourse Communication Studies, 26(1), 3-37.

Burnard, P. (1991). A method of analysing interview transcripts in qualitative research. Nurse Education Today, 11(6), 461-466.

Caldas-Coulthard, C. R. (2008). Body branded: multimodal identities in tourism advertising. Journal of Language and Politics, 7(3), 451-470.

Callan, R. J., \& Fearon, R. (1997). Town house hotels - an emerging sector. International Journal of Contemporary Hospitality Management, 9(4), 168-175.

Chaidaroon, S. S. (2003). When shyness is not incompetence: A case of Thai communication competence. Asian Approaches to Human Communication. 294-306. Retrieved from 2 April, 2016, from

https://www.researchgate.net/publication/242488177 When Shyness Is Not Incompeten ce A Case of Thai Communication Competence

Chan, C. (2012). Lodging subsector report: boutique hotels. Retrieved 20 February, 2014, from https://atrium.lib.uoguelph.ca/xmlui/handle/10214/3587

Chang, T.C., \& Teo, P. (2009). The shophouse hotel: vernacular heritage in a creative city. Urban Studies, 46 (2), 341-367.

Chanpetch, J. (2011). A genre based analysis on rhetorical pattern of travel articles in Tropical Magazine. Unpublished masters thesis, Kasetsart University, Bangkok, Thailand.

Chapman, C (2010). Color theory for designers, Part 1: The meaning of color. Retrieved 14 April, 2015, from https://www.smashingmagazine.com/2010/01/color-theory-for-designerspart-1-the-meaning-of-color

Chen, H. (2008). Learning in new times: writing through the "eyes of genre". Faculty of EducationPapers, 45. 
Chiavetta, E. (2006). A genre analysis of 'how to' feature articles in British gardening magazines. In V.K. Bhatia \& M. Gotti (Eds.), Explorations in specialised genres (pp. 55-175). Bern: Peter Lang.

Christie, F. (1987). Young children's writing: From spoken to written genre. Language and Education, 1(1), 3-13.

Christie, F., \& Martin, J. R. (1997). Genre and institutions: Social processes in the workplace and school. London: Continuum.

Chung, T., \& Law, R. (2003). Developing a performance indicator for hotel websites. International Journal of Hospitality Management, 22(1), 119-125.

Coates, J. (1983). The semantics of the modal auxiliaries. London: Croom Helm.

Coe, R. M. (2002). The new rhetoric of genre: Writing political briefs. In A.M. Johns (Ed.), Genre in the classroom: Multiple perspectives (pp. 197-207). London: Lawrence Erlbaum Associates.

Conrad, S. (2002). Corpus linguistic approaches for discourse analysis. Annual Review of Applied Linguistics, 22(1), 75-95.

Cook, G. (2001). The discourse of advertising. London: Routledge.

Cook, G., Reed, M., \& Twiner, A. (2009). "But it's all true!": commercialism and commitment in the discourse of organic food promotion. Text \& Talk - An Interdisciplinary Journal of Language, Discourse Communication Studies, 29(2), 151-173.

Corbetta, P. (2003). Social research: Theory, methods and techniques: Sage.

Covelli, M. J. (2005). Boutique hotels are getting new interest. The Buffalo business first afternoon. Retrieved 20 February, 2014 from http://www.bizjournals.com/buffalo/stories/2005/01/31/focus4.html?page=all

Crossley, S. (2007). A chronotopic approach to genre analysis: An exploratory study. English for Specific Purposes, 26(1), 4-24.

Cupples, L. (2002). The structural characteristics and on-line comprehension of experiencer-verb sentences. Language and Cognitive Processes, 17(2), 125-162.

Dann, G. M. (1996). The language of tourism: a sociolinguistic perspective. Wallingford, UK: CAB International.

De Bortoli, M., \& Maroto, J. (2001). Colours across cultures: Translating colours in interactive marketing communications. European Languages and the Implementation of Communication and Information Technologies, 1-27.

De Klumbis, D. F. (2005). Seeking the "ultimate hotel experience". Retrieved 12 January, 2015, from http://www.esade.edu/cedit2003/pdfs/danielafreund.pdf 
Devitt, A. (2004). Writing genres. Carbondale, IL: Southern Illinois University Press.

DiCicco-Bloom, B., \& Crabtree, B. F. (2006). The qualitative research interview. Medical Education, 40(4), 314-321.

Doolin, B., Burgess, L., \& Cooper, J. (2002). Evaluating the use of the Web for tourism marketing: a case study from New Zealand. Tourism Management, 23(5), 557-561.

Dörnyei, Z. (2011). Research methods in applied linguistics. Oxford: Oxford University Press.

Dudley-Evans, T. (1994). Genre analysis: An approach to text analysis for ESP. Advances in written text analysis (pp. 219-228). London, New York: Routledge.

Dudley-Evans, T. (2000). Genre analysis: a key to a theory of ESP? Ibérica: Revista de la Asociación Europea de Lenguas para Fines Específicos (AELFE)(2), 3-11.

Ellis, R., Johnson, K. E., Henry, A., \& Roseberry, R. L. (1998). An evaluation of a genre-based approach to the teaching of EAP/ESP writing. TESOL Quarterly, 32(1), 147-156.

Djonov, E. N. (2008). Children's website structure and navigation. In L. Unsworth (Eds.), Multimodal Semiotics: Functional Analysis in Contexts of Education (pp. 216-236). London: Continuum International.

Djonov, E., \& Knox, J. (2014). How-to-Analyze Webpages. In N.Sigrid \& M. C. Daniela (Eds.), Texts, Images, and Interactions: A Reader in Multimodality (171-194). Berlin: Gruyter.

Erkutlu, H. V., \& Chafra, J. (2006). Relationship between leadership power bases and job stress of subordinates: example from boutique hotels. Management Research News, 29(5), 285-297.

Feez, S. (2002). Heritage and innovation in second language education. Genre in the classroom: Multiple perspectives, 43-72.

Ferreira, F. (1994). Choice of passive voice is affected by verb type and animacy. Journal of Memory and Language, 33(6), 715-736.

Figueiredo, D. (2010). Context, register and genre: Implications for language education. Revista Signos, 43(1), 119-141.

Flowerdew, J., (2002). Genre in the classroom: A linguistic approach. In A.M. Johns (Ed.), Genre in the classroom: Multiple perspectives (pp. 91-102). London: Lawrence Erlbaum Associates.

Flowerdew, L. (2004). The argument for using English specialized corpora to understand academic and professional language. In U. Connor \& T. Upton (Eds.), Discourse in the professions: Perspectives from corpus linguistics (pp. 11-33). Amsterdam: John Benjamins. 
Flowerdew, J., \& Wan, A. (2006). Genre analysis of tax computation letters: How and why tax accountants write the way they do. English for Specific Purposes, 25(2), 133-153.

Flowerdew, J., \& Wan, A. (2010). The linguistic and the contextual in applied genre analysis: The case of the company audit report. English for Specific Purposes, 29(2), 78-93.

Flowerdew, L. (2005). An integration of corpus-based and genre-based approaches to text analysis in EAP/ESP: Countering criticisms against corpus-based methodologies. English for Specific Purposes, 24(3), 321-332.

Ford, J. B., Voli, P. K., Honeycutt Jr, E. D., \& Casey, S. L. (1998). Gender role portrayals in Japanese advertising: A magazine content analysis. Journal of Advertising, 27(1), 113-124.

Francesconi, S. (2011). Images and writing in tourist brochures. Journal of Tourism and Cultural Change, 9(4), 341-356.

Francesconi, S. (2012). Generic integrity and innovation in tourism texts in English: Tangram Ediz. Scientifiche.

Francesconi, S. (2014). Reading Tourism Texts: A Multimodal Analysis. Bristol: Channel View Publications.

Franzosi, P. R (2009). Content analysis. In Hardy, M. A., \& Bryman, A. (Eds.), Handbook of data analysis (pp. 547-565). Los Angeles: Sage.

Freedman, A. (1993). Show and tell? The role of explicit teaching in the learning of new genres. Research in the Teaching of English, 27(3), 222-251.

Freedman, A., \& Medway, P. (1994). Genre in the new rhetoric. London: Taylor \& Francis.

Fuertes-Olivera, P. A., Velasco-Sacristán, M., Arribas-Baño, A., \& Samaniego-Fernández, E. (2001). Persuasion and advertising English: Metadiscourse in slogans and headlines. Journal of pragmatics, 33(8), 1291-1307.

Giannopoulos, A. A., \& Mavragani, E. P. (2011). Traveling through the web: A first step toward a comparative analysis of European national tourism websites. Journal of Hospitality Marketing \& Management, 20(7), 718-739.

Gilbert, D., \& Powell-Perry, J. (2001). Exploring developments in web based relationship marketing within the hotel industry. Journal of Hospitality \& Leisure Marketing, 9(3-4), 141-159.

Götz, I. (2015). What are the differences and similarities of boutique and lifestyle hotels for professionals, consumers and students? Unpublished bachelor's thesis, University of Applied Sciences, Haaga-Helia.

Govers, R., \& Go, F. M. (2005). Projected destination image online: Website content analysis of pictures and text. Information Technology \& Tourism, 7(2), 73-89.

Gray, D. E. (2009). Doing research in the real world. London: Sage Publications Limited. 
Hale, C. (2012). The Pleasure and Perils of the Passive. Retrieved 20 February, 2014 from http://opinionator.blogs.nytimes.com/2012/04/30/the-pleasures-and-perils-of-thepassive/? $r=0$

Hall, C. M., \& Kearsley, G. W. (2001). Tourism in New Zealand: an introduction. Australia: Oxford University Press.

Halliday, M. A. (1985). An introduction to functional grammar. London: Arnold.

Halliday, M. A. K. (1978). Language as social semiotic. London: Arnold.

Halliday, M. A. (1994). An introduction to functional grammar. London: Arnold.

Halliday, M., \& Matthiessen, C. (2004). Introducing functional grammar. New York: Edward Arnold. Harwood, N. (2005). 'We do not seem to have a theory... The theory I present here attempts to fill this gap': Inclusive and exclusive pronouns in academic writing. Applied Linguistics, 26(3), 343-375.

Hasan, R. (1989). The structure of a text. In M. A. K. Halliday, \& R. Hasan. Language, context, and text: Aspects of language in a social-semiotic perspective. Oxford: Oxford University Press.

Henderson, J. C. (2011). Hip heritage: The boutique hotel business in Singapore. Tourism and Hospitality Research, 11(3), 217-223.

Henderson, J. C., Liew, G., Ong, J., \& Quek, B. (2013). The use of urban built heritage for boutique hotels: examples from Malaysia and Singapore. Tourism Review International, 17(2), 63-74.

Henry, A. (2007). Evaluating language learners' response to web-based, data-driven, genre teaching materials. English for Specific Purposes, 26(4), 462-484.

Henry, A., \& Roseberry, R. L. (1996). A corpus-based investigation of the language and linguistic patterns of one genre and the implications for language teaching. Research in the Teaching of English, 30, 472-489.

Henry, A., \& Roseberry, R. L. (2001). A narrow-angled corpus analysis of moves and strategies of the genre: Letter of application. English for Specific Purposes, 20, 153-167.

Henry, A., \& Roseberry, R. L. (2001). A narrow-angled corpus analysis of moves and strategies of the genre:'Letter of Application'. English for Specific Purposes, 20(2), 153-167.

Herbozo, S., Tantleff-Dunn, S., Gokee-Larose, J., \& Thompson, J. K. (2004). Beauty and thinness messages in children's media: A content analysis. Eating Disorders, 12(1), 21-34.

Hsieh, H.-F., \& Shannon, S. E. (2005). Three approaches to qualitative content analysis. Qualitative Health Research, 15(9), 1277-1288.

Hiippala, T. (2007). Helsinki: A multisemiotic analysis of tourist brochures. Unpublished master's thesis, University of Helsinki. 
Hiippala, T. (2012). The localisation of advertising print media as a multimodal process. In W. L. Bowcher (Ed.), Multimodal texts from around the world (pp. 97-122). London: Palgrave Macmillan.

Hoang, T. V. Y., \& Rojas-Lizana, I. (2015). Promotional discourse in the websites of two Australian universities: A discourse analytic approach. Cogent Education, 2(1), 1-19.

Hofacker, C. F., \& Murphy, J. (1998). World Wide Web banner advertisement copy testing. European Journal of Marketing, 32(7/8), 703-712.

Holmes, R. (1997). Genre analysis, and the social sciences: An investigation of the structure of research article discussion sections in three disciplines. English for Specific Purposes, 16(4), 321-337.

Hou, H.-I. (2012). A corpus approach to discourse analysis of newspaper restaurant reviews: A preliminary analysis. Studies in Literature and Language, 5(3), 95-100.

Hyland, K. (2001). Bringing in the reader addressee features in academic articles. Written Communication, 18(4), 549-574.

Hyland, K. (2002). Authority and invisibility: Authorial identity in academic writing. Journal of Pragmatics, 34(8), 1091-1112.

Hyland, K. (2004). Disciplinary discourses: Social interactions in academic writing: University of Michigan Press.

Hyland, K. (2005). Stance and engagement: A model of interaction in academic discourse. Discourse Studies, 7(2), 173-192.

Hyland, K. (2007). Genre pedagogy: Language, literacy and L2 writing instruction. Journal of Second Language Writing, 16(3), 148-164.

Hyland, K. (2012). Disciplinary identities: Individuality and community in academic discourse. Cambridge: Cambridge University Press.

Hyon, S. (1996). Genre in three traditions: Implications for ESL. TESOL Quarterly, 30(4), 693-722. Janoschka, A. (2004). Web advertising: new forms of communication on the Internet. Philadephia: John Benjamins Publishing.

Jeong, M., \& Choi, J. (2005). Effects of picture presentations on customers' behavioral intentions on the web. Journal of Travel \& Tourism Marketing, 17(2-3), 193-204.

Jewitt, C. (2009a). An introduction to multimodality. In Carrey. J (Ed.), The Routledge handbook of multimodal analysis (pp. 14-27). New York: Routledge.

Jewitt, C. (2009b). Different approaches to multimodality. In J. Carrey (Ed.), The Routledge handbook of multimodal analysis (pp. 28-39). New York: Routledge. 
Jewitt, C., \& Oyama, R. (2001). Visual meaning: a social semiotic. In T. Van Leeuwen \& C. Jewitt (Eds.), Handbook of visual analysis (134-156). London: Sage.

Johari, D. M., \& Ali, M. A. (2015). The business web genre: a genre analysis on the websites of selected Malaysian small and medium enterprise (SMEs). Isamlamic perspective relating to business, arts, culture and communication, 383-404.

Johns, A. M. (1997). Text, role, and context: Developing academic literacies. New York: Cambridge University Press.

Johns, A. M. (2002). Genre in the classroom: Multiple perspectives. London: Lawrence Erlbaum Associates.

Johns, A. M., Bawarshi, A., Coe, R. M., Hyland, K., Paltridge, B., Reiff, M. J., \& Tardy, C. (2006). Crossing the boundaries of genre studies: Commentaries by experts. Journal of Second Language Writing, 15(3), 234-249.

Johnson-Laird, P. N. (1968). The choice of the passive voice in a communicative task. British Journal of Psychology, 59(1), 7-15.

Jones, D. L., Day, J., \& Quadri, D. (2013). Emerging definitions of boutique and lifestyle hotels: A Delphi study. Journal of Travel \& Tourism Marketing, 30(7), 715-731.

Kanoksilapatham, B. (2003). A corpus-based investigation of scientific research articles: Linking move analysis with multidimensional analysis: Georgetown University.

Kanoksilapatham, B. (2005). Rhetorical structure of biochemistry research articles. English for Specific Purposes, 24(3), 269-292.

Kanoksilapatham, B. (2007a). Rhetorical moves in biochemistry research articles. In Biber, D., Connor, U., \& Upton T. (Eds.), Discourse on the move: Using corpus analysis to describe discourse structure (pp. 73-119). Amsterdam and Philadephia: John Benjamins Publishing Company.

Kanoksilapatham, B. (2007b). Writing scientific research articles in Thai and English: Similarities and differences. Silpakorn University International Journal, 7, 172-203.

Kathpalia, S. S. (1992). A genre analysis of promotional texts. Singapore: National University of Singapore.

Kay, H., \& Dudley-Evans, T. (1998). Genre: What teachers think. ELT Journal, 52(4), 308-314.

Khosravi, S., Malek, A., \& Ekiz, E. (2014). Why tourists are attracted to boutique hotels: case of Penang Island, Malaysia. Journal of Hospitality \& Tourism, 12(1).

Khunnawut, S. (2009). English as a Foreign Language in the Thai Higher Education Context: Investigations Into Teaching and Learning Styles and Strategies, and Other Factors. Unpublished PhD thesis, Technishen Universitat Dresden, Germany. 
Klankanna, T. (2007). A Genre Analysis of Hotel Homepages. Unpublished master's thesis, Kasetsart University, Bangkok, Thailand.

Kleindl, B. A. 2003. Strategic electronic marketing: Managing e-business. Ohio: South-Western College Publishing.

Knox, J. (2007). Visual-verbal communication on online newspaper home pages. Visual Communication, 6(1), 19-53.

Kress, G., \& van Leeuwen, T. (1996). Reading images: The grammar of visual design. London: Routledge.

Kress, G., \& Van Leeuwen, T. (1998). Front pages: (The critical) analysis of newspaper layout. In A. Bell \& P. Garrett (Eds.), Approaches to media discourse (pp. 186-219). Oxford: Blackwell.

Kress, G. R., \& van Leeuwen, T. (2006). Reading images: The grammar of visual design. New York: Psychology Press.

Labrador, B., Ramón, N., Alaiz-Moretón, H., \& Sanjurjo-González, H. (2014). Rhetorical structure and persuasive language in the subgenre of online advertisements. English for Specific Purposes, 34, 38-47.

Leech, G.N. (1996). English in advertising: A linguistic study of advertising in Great Britain. London: William Clowes and Sons, Limited.

Lemke, J. L. (2002). Travels in hypermodality. Visual Communication, 1(3), 299-325.

Lewin, A., Fine, J. \& Young, L. (2001). Expository Discourse: A Genre-based Approach to Social Science Research Texts. London/New York: Continuum.

Li, L. (2004). Theoretical base and problems in Business English teaching in China. English for Specific Purposes World, Online Journal for Teachers, 3(2), 8.

Li, M. (2009). The stylistic analysis of the magazine advertisement----Atkins Chocolate Chip Granola Bar. Asian Social Science, 5(9), 63.

Lim, W. M., \& Endean, M. (2009). Elucidating the aesthetic and operational characteristics of UK boutique hotels. International Journal of Contemporary Hospitality Management, 21(1), 3851.

Lindgaard, G., Fernandes, G., Dudek, C., \& Brown, J. (2006). Attention web designers: You have 50 milliseconds to make a good first impression! Behaviour \& Information Technology, 25(2), 115-126.

Lock, G. (1996). Functional English grammar: An introduction for second language teachers: Cambridge University Press.

Machin, D. (2007). Introduction to multimodal analysis. London: Bloomsbury. 
Maci, S. (2007). Virtual touring: The web-language of tourism. Linguistica e Filologia, 25, 41-65.

Macken-Horarik, M. (2002). Something to shoot for: A systemic functional approach to teaching genre in secondary school science. Genre in the classroom: Multiple perspectives, 17-42.

Mackey, A., \& Gass, S. M. (2005). Second language research: Methodology and design. Mahwah, NJ: Lawrence Erlbaum.

Manca, E. (2008). From phraseology to culture: Qualifying adjectives in the language of tourism. International Journal of Corpus Linguistics, 13(3), 368-385.

Marco, M. J. L. (2002). A genre analysis of corporate home pages. LSP and Professional Communication (2001-2008), 2(1).

Martin, J. R. (2001). Giving the game away: explicitness, diversity and genre-based literacy in Australia: (np) Retrieved 20 May, 2016 from http://www.isfla.org/Systemics/Print/MartinPapers/BC-2001Giving\%20the\%20game\%20way\%20explicitness\%20diversity\%20and\%20genre.PDF

Martin, J. R. (1997). Analysing genre: Functional parameters. In F. Christie \& J. R. Martin (Eds.), Genre and institutions: Social processes in the workplace and school (pp. 3-39). London: Cassell.

Martin, J. R. (2001). Language, register and genre. In A. Burns \& C. Coffin (Eds.), Analysing English in a global context: A reader (pp. 149-166). London: Routledge.

Martin, J. R., Christie, F., \& Rothery, J. (1987). Social processes in education: A reply to Sawyer and Watson (and others). In I. Reid (Ed.), The place of genre in learning: Current debates (pp. 3545). Geelong, Australia: Centre for Studies in Literary Education.

Martinec, R. (2000). Types of process in action. Semiotica, 130(3-4), 243-268.

Mauranen, A. (1993). Contrastive ESP rhetoric: Metatext in Finnish-English economics texts. English for Specific Purposes, 12(1), 3-22.

Mauranen, A. (2012). Exploring ELF: Academic English shaped by non-native speakers: Cambridge University Press.

Maynard, M., \& Tian, Y. (2004). Between global and glocal: Content analysis of the Chinese web sites of the 100 top global brands. Public Relations Review, 30(3), 285-291.

McIntosh, A. J., \& Siggs, A. (2005). An exploration of the experiential nature of boutique accommodation. Journal of Travel Research, 44(1), 74-81.

McKenney, S. (2015). The boutique and lifestlye hotel report 2015. Retrieved 20 May, 2015 from http://hotelanalyst.co.uk/wp-content/uploads/sites/2/2015/05/boutique-hotel-report2015-sample.pdf 
McLellan, E., MacQueen, K. M., \& Neidig, J. L. (2003). Beyond the qualitative interview: data preparation and transcription. Field Methods, 15(1), 63-84.

Medina, A. B., Medina, M. J. R., Medina, L. E. B., \& García, L. J. L. (2005). Analysing digital genres: function and functionality in corporate websites of computer hardware. Ibérica: Revista de la Asociación Europea de Lenguas para Fines Específicos (AELFE)(9), 123-147.

Mencher, M. (1996). Basic media writing. United States: McGraw-Hill.

Meng, A. P. K. (2004). Making history in From Colony to Nation: A multimodal analysis of a museum exhibition in Singapore. In K. L. O'Halloran (Ed.), Multimodal Discourse Analysis: SystemicFunctional Linguistics (pp. 28-54). London and New York: Continuum.

Meyer, C. F. (2002). English corpus linguistics: An introduction. Cambridge: Cambridge University Press.

Mill, R. C., \& Morrison, A. M. (2002). The tourism system. Prentice Hall, Englewood Cliffs, NJ: Kendall Hunt.

Miller, C. R. (1984). Genre as social action. Quarterly Journal of Speech, 70, 151-176.

Ministry of Business Innovation and Employment. (2012). The most visited regions in New

Zealand. Retrieved 15 January, 2013, from

http://www.infotools.com/MEDVizulite/?PageLocation=MED/CubePages/MED_IVS_TravelP atterns RTOsTravelledTo table

Mintel Group Ltd (2011). Boutique hotels in the US-July 2011. London, United Kingdom: Mintel Group Ltd.

Mittal, B. (1999). The advertising of services meeting the challenge of intangibility. Journal of Service Research, 2(1), 98-116.

Mongkholijuck, C. (2008). A genre analysis of tourist attraction leaflet produced and distributed in Thailand in 2004. Unpublished master's thesis, Kasetsart University, Bangkok, Thailand.

Morgan, N., Pritchard, A., \& Pride, R. (2002). Destination branding: creating the unique destination proposition. Oxford: Elsevier Butterworth-Heinemann.

Moya Guijarro, J., \& Pinar Sanz, M. J. (2008). Compositional, interpersonal and representational meanings in a children's narrrative: A multimodal discourse analysis. Journal of Pragmatics, 40(9), 1601-1619.

Mulken, M., \& van der Meer, W. (2005). Are you being served?: A genre analysis of American and Dutch company replies to customer inquiries. English for Specific Purposes, 24(1), 93-109.

Munsters, W., \& de Klumbis, D. F. (2006). Culture as a component of the hospitality product. In D. Leslie \& M. Sigala, International cultural tourism, (pp. 26-39). Amsterdam: ButterworthHeinemann. 
Mur-Dueñas, P. (2013). Spanish scholars' research article publishing process in English-medium journals: English used as a lingua franca? Journal of English as a Lingua Franca, 2(2), 315340.

Myers, G. (1994). Words in ads. London: Edward Arnold.

Nielsen, J., Tahir, M., \& Tahir, M. (2002). Homepage usability: 50 websites deconstructed (Vol. 50). USA: New Riders Publishing.

Ning, Z.-y. (2008). A genre-based analysis of English research article abstracts and the linguistic feature of personal pronouns for financial economics. US-China Education Review, 5(7), 6265.

Nobles, H. (2006). Is 'Mega Boutique hotel' an Oxymoron. Retrieved 20 January, 2015 from http://www.hotel-online.com/News/PR2006_4th/Dec06_MegaBoutique.html

Nodelman, P. (1988). Words about pictures: The narrative art of children's picture books. USA: University of Georgia Press.

Norris, S. (2004). Analyzing multimodal interaction: A methodological framework. New York: Routledge.

Nunan, D., \& Bailey, K. M. (2009). Exploring second language classroom research: A comprehensive guide. Boston: Heinle Cengage Learning.

O'Connor, P., \& Frew, A. J. (2004). An evaluation methodology for hotel electronic channels of distribution. International Journal of Hospitality Management, 23(2), 179-199.

O'Guinn, T. C., Allen, C. T., \& Semenik, R. J. (1998). Advertising. Cincinnati: Southwestern College Publishing.

O'Halloran, K.L. (2008). Systemic functional-multimodal discourse analysis (SF-MDA): Constructing ideational meaning using language and visual imagery. Visual Communication, 7, 443-475.

O'Halloran, K.L. (in press). Multimodal Analysis and Digital Technology. In A. Baldry and E. Montagna (Eds.), Interdisciplinary perspectives on multimodality: Theory and practice. Campobasso: Palladino. Retrieved 15 April, 2013 from http://multimodalanalysisab.org/ docs/Multimodal\%20Analysis\%20and\%20Digital\%20Tech nology.pdf

Olga, A., 2009. The alternative hotel market. International Conference on Management Science \& Engineering. Moscow, Russia.

Oyama, R. (1999). Visual semiotics in a cross-cultural perspective: a study of visual images in Japanese and selected British advertisements. Unpublished PhD thesis. London: Institute of Education (University of London). 
Paltridge, B. (1997). Genre, frames and writing in research settings. Amsterdam and Philadelphia: John Benjamins Publishing.

Paltridge, B. (2002). Genre, text type, and the English for Academic Purposes (EAP) classroom. Genre in the classroom: Multiple perspectives, 73-90.

Pare, A. \& Smart, G. (1994). Observing genres in action: towards a research methodology. In A. Freedman \& P. Medway (eds.), Genre and the new rhetoric (pp 146-154). London: Taylor and Francis.

Parets, R. (2002). Getting the word out: profiting from smart Internet marketing isn't just for the big boys. Lodging Magazine, 8, 37-38.

Parkinson, J., \& Adendorff, R. (2004). The use of popular science articles in teaching scientific literacy. English for Specific Purposes, 23(4), 379-396.

Parkinson, J., \& Adendorff, R. (2005). Variable discursive constructions of three genres of science. Southern African linguistics and applied language studies, 23(3), 281-303.

Peacock, M. (2002). Communicative moves in the discussion section of research articles. System, 30(4), 479-497.

Perdue, R. R. (2002). Internet site evaluations: the influence of behavioral experience, existing images, and selected website characteristics. Journal of Travel \& Tourism Marketing, 11(2-3), 21-38.

Phongsuwan, T. (2009). A genre analysis of homepages of serviced apartments in Bangkok. Unpublished master's thesis, Kasetsart University, Bangkok, Thailand.

Pierini, P. (2009). Adjectives in tourism English on the Web: A corpus-based study. Círculo de lingüística aplicada a la comunicación(40), 4.

Pitchsinee, S. (1995). Persuasive communication for tourists through advertising in brochure: A look at national tourist organization. Unpublished master's thesis, Chulalongkorn University, Bangkok, Thailand.

Pollach, I. (2006). Electronic word of mouth: A genre analysis of product reviews on consumer opinion web sites. Proceedings of the 39th Annual Hawaii International Conference on System Sciences.

Quirk, R., Greenbaum, S., Leech, G., Svartvik, J., \& Crystal, D. (1985). A comprehensive grammar of the English language (Vol. 397). Cambridge: Cambridge University Press.

Rabontu, C. I., \& Niculescu, G. (2009). Boutique hotels-new appearances in hotel industry in Romania. Annals of the University of Petrosani, Economics, 9(2), 209-214.

Rahman, M. M. (2011). Genre-based writing instruction: implications in ESP classroom. English for Specific Purposes World, 33(11), 1-9. 
Rajandran, K. (2009). Multimodality in Malaysian commercial bank brochures. Círculo de lingüística aplicada a la comunicación(37), 3.

Rasmeenin, C. (2006). A structural move analysis of MA thesis discussion sections in applied linguistics. Mahidol University.

Reppen, R. (2004). Academic language: An exploration of university classroom and textbook language. Discourse in the professions. Perspectives from corpus linguistics, 65-87.

Rogerson, J. M. (2010). The boutique hotel industry in South Africa: definition, scope, and organization. Paper presented at the Urban Forum.

Rounds, P. L. (1987). Multifunctional personal pronoun use in an educational setting. English for Specific Purposes, 6(1), 13-29.

Ruffini, M. F. 2001. Blueprint to Develop a Great Web Site. Journal (Online). Retrieved 20 August 2007 from http://thejournal.com/articles/2001/03/01/blueprint-to-develop-a-great-web-site.aspx

Ruiz-Garrido, M. F., \& Saorín-Iborra, A. M. (2013). Language for tourism. The Encyclopedia of Applied Linguistics.

Sadler, G. R., Lee, H. C., Lim, R. S. H., \& Fullerton, J. (2010). Recruitment of hard-to-reach population subgroups via adaptations of the snowball sampling strategy. Nursing \& Health Sciences, 12(3), 369-374.

Salmani Nodoushan, M. A. (2012). A structural move analysis of discussion sub-genre in applied linguistics. Paper presented at the International Conference on Languages, E-Learning and Romanian Studies.

Salmani Nodoushan, M. A., \& Montazeran, H. (2012). The book review genre: A structural move analysis. International Journal of Language Studies, 6(1), 1-30.

Samaru-Charles, L. (2001). Rule-Breaking in the Language of Advertising. Unpublished senior thesis. n.p. Retrieved 15 May, 2015, from

\section{http://thesis.haverford.edu/dspace/bitstream/handle/10066/10849/Samaru-}

\section{Charles thesis 2002.pdf?sequence $=1$}

Samraj, B. (2004). Discourse features of the student-produced academic research paper: Variations across disciplinary courses. Journal of English for Academic Purposes, 3, 5-22.

Santini, M. (2007). Characterizing genres of web pages: Genre hybridism and individualization. Paper presented at the 40th Annual Hawaii International Conference on System Sciences.

Scollon, R., \& Scollon, S. B. (2003). Discourses in place: Language in the material world. London: Routledge.Baroni, M., \& Ueyama, M. (2006). Building general-and special-purpose corpora 
by Web crawling. Proceedings of the 13th NIJL International Symposium, Language Corpora: Their Compilation and Application (pp. 31-40).

Scowsill, D. (2015). Travel \& tourism economic impact 2015 world. Retrieved 22 Jan, 2016, from https://www.wttc.org//media/files/reports/economic\%20impact\%20research/regional\%202015/world2015.pdf

Shepherd, M., \& Watters, C. (1999). The functionality attribute of cybergenres. Paper presented at the 1999 HICSS-32. Proceedings of the 32nd Annual Hawaii International Conference on Systems Sciences.

Sigala, M., \& Leslie, D. (2006). Conclusion, The future of the past: visions and trends for cultural tourism sector. International cultural tourism: management, implications and cases, 234240). Oxford: Elsevier.

Sinaga, G.P. (2012). The interpersonal strategies of hotel and apartment advertisements in Now! Jakarta life in the capital magazine. English Language Teaching in Indonesia (ELTI) Journal, 1 (1), 47-59.

Singh, N., Zhao, H., \& Hu, X. (2005). Analyzing the Cultural Content of Web Sites: A Cross-National Comparision of China, India, Japan, and US. International Marketing Review, 22(2), 129-146.

Steffens, N. K., \& Haslam, S. A. (2013). Power through 'us': Leaders' use of we-referencing language predicts election victory. PloS one, 8(10), e77952

Strannegård, L., \& Strannegård, M. (2012). Works of art: Aesthetic ambitions in design hotels. Annals of Tourism Research, 39(4), 1995-2012.

Stubbs, M. (1996). Text and corpus analysis: computer assisted studies of language and culture. Edinburgh: Blackwell.

Suen, A. (2008) Self-representation of five star hotels: A digital genre analysis of hotel websites. Paper at the Partnerships in Action: Research, Practice \& Training. Inaugural Conference of the Asia-Pacific Rim LSP and Professional Communication Association. City University of Hong Kong. December 8-10, 2008.

Suen, A. (2009). Self-representation of five star hotels: A digital genre analysis of hotel homepages. Language for Professional Communication: Research, Practice and Training, 111-130.

Suen, O. Y. (2013). Hotel websites as corporate communication (Doctoral thesis, The Hong Kong Polytechnic University). Retrieved 22 January, 2015, from http://ira.lib.polyu.edu.hk/bitstream/10397/6142/2/b26158796 ir.pdf

Swales, J. (1990). Genre analysis: English in academic and research settings. Cambridge: Cambridge University Press. 
Swales, J. (2004). Research genres: Explorations and applications. Cambridge: Cambridge University Press.

Swales, J. M., \& Lindemann, S. (2002). Teaching the literature review to international graduate students. In A. Johns (Ed.), Genre in the classroom: Multiple perspectives (pp. 105-119). Mahwah, NJ: Lawrence Erlbaum.

Swales, J. M., Ahmad, U. K., Chang, Y.-Y., Chavez, D., Dressen, D. F., \& Seymour, R. (1998). Consider this: The role of imperatives in scholarly writing. Applied Linguistics, 19(1), (pp.97-121).

Tardy, C. M. (2013). Genre analysis. In K. Hyland \& B. Paltridge (Eds.), Continuum companion to discourse analysis (pp. 54-68). London: Bloomsbury Publishing.

Temphairojana, P. (2017, January 5). Thailand tourist numbers rise nearly nine percent in 2016. Reuters. Retrieved from http://www.reuters.com/article/us-thailand-tourism/thailandtourist-numbers-rise-nearly-nine-percent-in-2016-idUSKBN14P0QF

The Ministry of Tourism (2015). New Zealand Tourism Strategy 2015. Retrieved 15 May, 2016, from http://www.mbie.govt.nz/publications-research/publications/tourism/nz-tourism-strategy2015.pdf

Tidtichumrernporn, T. (2012). Lifestyle segmentation for boutique accommodation in relation to the service quality and customer. Paper presented at the 17th Asia-Pacific Decision Sciences Institute Conference.

Timothy, D., \& Teye, V. (2009). Tourism and the lodging sector. Oxford: Butterworth-Heinemann.

Tisapramotkul, O. (2007). A genre-based study of English recruitment advertisements in Thai and British daily newspapers. Unpublished master's thesis, Chulalongkorn University, Bangkok, Thailand.

Top ten destinations in Thailand 2013 by Trip Advisor. Retrieved 15 January, 2013, from http://www.thaitravelblogs.com/2013/05/bangkok-wins-best-in-asia-award-2013/

Top ten destinations in Thailand 2013 by NIDA Poll. Retrieved 15 January, 2013, from http://travel.kapook.com/view42726.html

Tourism Authority of Thailand. (2012). The most visited regions in Thailand. Retrieved 12 December, 2012 from http://www.tourism.go.th/tourism/th/home/tourism.php.

Unsworth, L. (2001). Teaching multiliteracies across the curriculum: changing contexts of texts and image in classroom practice. Philadelphia: Open University Press.

Unsworth, L., \& Wheeler, J. (2002). Re-valuing the role of images in reviewing picture books. Reading, 36(2), 68-74. 
Upton, T. A., \& Connor, U. (2001). Using computerized corpus analysis to investigate the textlinguistic discourse moves of a genre. English for Specific Purposes, 20(4), 313-329.

Van Hartesvelt, M. (2006). Building a better boutique hotel. Lodging Hospitality, 62(14), 32-44.

Van Leeuwen, T. (2005). Introduction to social semiotics. London: Routledge.

Vergaro, C. (2004). Discourse strategies of Italian and English sales promotion letters. English for Specific Purposes, 23(2), 181-207.

Vine, B. (2004). Modal verbs in New Zealand English directives. Nordic Journal of English Studies, $3(3), 205-220$.

Virtanen, T., \& Halmari, H. (2005). Persuasion across genres: Emerging perspectives. In H. Halmari \& T. Virtanen, Persuasion Across Genres: A linguistic approach (pp. 3-24). Amsterdam and Philadelphia: John Benjamins Publishing Company.

Vithal, B., \& Gillespie, M. (2011). Boutique Hotels: The Ulitimate Chameleon. Journal of the KwazuluNatal Institute for Architecture, 3, 2. Retrieved 12 December, 2015 from http://kzniajournal.org.za/sites/default/files/KZNIA-2011-36-3.pdf

Wang, T. (2013). A Case Study of Customer Motivation in Boutique Hotels in Xiamen, China, Using Push-Pull Theory (Graduate thesis, lowa State University). Retrieved 12 December, 2015 from http://lib.dr.iastate.edu/cgi/viewcontent.cgi?article=4630\&context=etd

Widdowson, H. (2002). Corpora and language teaching tomorrow. Paper presented at the Keynote lecture delivered at the 5th Teaching and Language Corpora Conference, Bertinoro, Italy.

Widodo, H. (2006). Designing a genre-based lesson plan for an academic writing course. English Teaching, 5(3), 173.

Wiriyapong, N. (2016, November 14). Swipe and go. Bangkok Post. Retrieved from http://m.bangkokpost.com/business/telecom/1134829/swipe-and-go

World Tourism Organization. (1999). Marketing tourism destinations online: strategies for the information age. World Tourism Organization.

Wuttanavrangkul, K. (2014). Thai tourism team presents "2015 Discover Thainess" campaign at WTM 2014. TATNEWS.org. Retrieved 3 March, 2016, from http://www.tatnews.org/thaitourism-team-presents-\%E2\%80\%9C2015-discover-thainess\%E2\%80\%9D-campaign-at$\underline{w t m-2014}$ 
Yakhontova, T. (2002). 'Selling'or 'Telling'? The issue of cultural variation in research genres. In J. Flowerdew (Ed.), Academic discourse (pp. 216-232). London, Routledge.

Yates, J., \& Orlikowski, W. J. (1992). Genres of organizational communication: A structurational approach to studying communication and media. Academy of Management Review, 17(2), 299-326.

Yeung, T. A., \& Law, R. (2006). Evaluation of usability: A study of hotel web sites in Hong Kong. Journal of Hospitality \& Tourism Research, 30(4), 452-473.

Yu, S., (2006). The Use of Personal Pronouns in Advertisements and Its Implications. Unpublished bachelor's thesis, Nanjing University, China. Retrieved 22 Jan, 2016, from cxrlinguistics.com

Zhou, S. (2012). 'Advertorials': A genre-based analysis of an emerging hybridized genre. Discourse \& Communication, 6(3), 323-346. 


\section{Appendices}

\section{Appendix 1: Ethics approval memorandum}

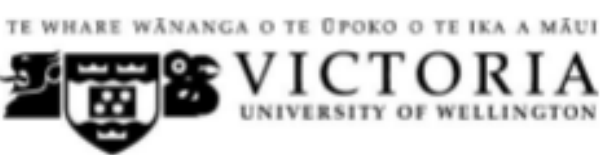

MEMORANDUM
Phone $\quad 0-4-4635676$

Fax $\quad 0-4-4635209$

Email Allison.birkmanovuw.ac.ns

\begin{tabular}{l|l}
\hline TO & Pakjira Leelertphan \\
\hline COPY TO & $\begin{array}{l}\text { Jean Parkinson } \\
\text { Averil Coxhead }\end{array}$ \\
\hline FROM & Dr Allison Kirkman, Convener, Human Ethics Committee \\
\hline
\end{tabular}

\begin{tabular}{l|l}
\hline DATE & 3 August 2013 \\
\hline PAGES & 1 \\
\hline
\end{tabular}

\begin{tabular}{l|l}
\hline SUBJECT & $\begin{array}{l}\text { Ethics Approval: } 19958 \\
\text { A comparative genre analysis of boutique hotel websites in } \\
\end{array}$ \\
& Thailand and New Zealand \\
\hline
\end{tabular}

Thank you for your application for ethical approval, which has now been considered by the Standing Committee of the Human Ethics Committee.

Your application has been approved from the above date and this approval continues until 18 December 2015. If your data collection is not completed by this date you should apply to the Human Ethics Committee for an extension to this approval.

Best wishes with the research.

Allison Kirkman

Human Ethics Committee

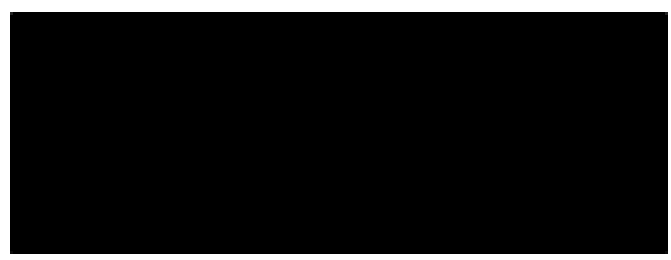




\title{
Appendix 2: Participant Consent Form for Hotels/Web Designers
}

\author{
VICTORIA UNIVERSITY OF WELLINGTON \\ Te Whare Wãnanga o te Ûpoko o te Ika a Mãui

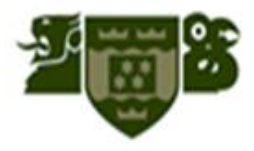

\section{Participant Consent Form for Hotels/Web Designers}

Project title: Analysis of boutique hotel websites in Thailand and New Zealand

\section{Researcher:}

Pakjira Leelertphan, School of Linguistics and Applied Language Studies, Victoria University of Wellington (VUW), New Zealand

NAME (please print clearly):

Please read the following statements carefully.

- I agree to participate in this research project.

- I have been provided with enough information about the nature and objectives of this research.

- I have had an opportunity to ask questions and have had them answered to my satisfaction.

- I understand that a pseudonym will be used.

- I understand that any information I provide will be kept at a secure location, and will only be available to the researcher and her supervisors

- I understand that I can withdraw myself or any data I have provided by $30^{\text {th }}$ of December 2014.

- I understand that all research notes and data will be destroyed five years after the conclusion of the research

- I understand that the information I provide will be used only by Pakjira Leelertphan and her supervisors for this research project, publications and presentation arising from this research

Signed:

Date:

I would like to receive a summary of the results of this research when it is completed YES/NO

Email address to which a summary can be sent: 


\title{
Appendix 3: Information sheet for hotels's owners/web designers
}

\author{
VICTORIA UNIVERSITY OF WELLINGTON \\ Te Whare Wānanga o te Ûpoko o te Ika a Mãui

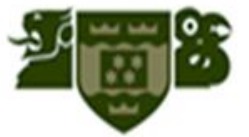

\section{Information sheet for hotels'owners/web designers}

Project title: Analysis of boutique hotel websites in Thailand and New Zealand

Researcher: Pakjira Leelertphan

School of Linguistics and Applied Language Studies, Victoria University of Wellington, New Zealand

I am a doctoral student at Victoria University of Wellington. As part of my PhD, I am analysing boutique hotel websites in Thailand and in New Zealand. In my study, I am investigating the language and images of these websites. I am also interested in the views of web designers and web readers.

I would like to interview you about your experience in writing boutique hotel websites. The interview will take no longer than one hour, at a time and place that is convenient to you.

In the interview, I would like to ask you about the language and the images you use in your websites. I will not use your real name when I write about the interview. I will send you a transcript of the interview so you can check it. If you have any concerns about confidential information, please do not hesitate to discuss your concerns with me, either in the interview or after.

The interview will be audiotaped. The data will be kept securely for up to five years before being destroyed. My supervisors will have access to the data. You can withdraw any time by email before $30^{\text {th }}$ December of 2014 . The results of this research may be published in academic journals or presented at academic conferences.

My study will help teachers and learners to develop writing materials for an English for Specific Purposes course in Travel and Tourism. This study will also provide directions for further study in English for Specific Purposes.

This research is supervised by Dr Jean Parkinson and Dr Keith Comer and has been approved by the Human Ethics Committee of VUW.

If you would like, I would be happy to provide you with a summary of the project, which will be sent to you at the end of the project.

If you have any questions about this study, please contact me at pakjira.leelertphan@vuw.ac.nz.

Thank you for your cooperation. 


\section{Contact details}

\section{Researcher:}

\section{$\underline{\text { Pakjira Leelertphan }}$}

New Zealand Address:

School of Linguistics and Applied Language Studies

Victoria University of Wellington

PO Box 600

Wellington 6140, New Zealand

Phone: +64 4463 5637/+64 212234876

E-mail: pakjira.leelertphan@vuw.ac.nz

\section{Dr Jean Parkinson}

School of Linguistics and Applied Language Studies

Victoria University of Wellington

PO Box 600

Wellington 6140

Phone: +64 4463 5233\#8009

E-mail: jean.parkinson@vuw.ac.nz
Thai Address:

99/288 Kingkeaw-Bangplee Road Bangpleeyai, Bangplee

Samutprakan 10540

Thailand

Phone: +66 816470732

E-mail: pakjira.leelertphan@vuw.ac.nz

Dr Keith Comer

School of Linguistics and Applied Language Studies

Victoria University of Wellington

PO Box 600

Wellington 6140

Phone: +64 4463 5233\#5629

E-mail: keith.comer@vuw.ac.nz 


\section{Appendix 4: Participant Consent Form for web readers}

\section{VICTORIA UNIVERSITY OF WELLINGTON \\ Te Whare Wānanga o te Ûpoko o te Ika a Mãui

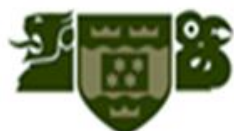

\section{Participant Consent Form for Web readers}

Project title: Analysis of boutique hotel websites in Thailand and New Zealand

\section{Researcher:}

Pakjira Leelertphan, School of Linguistics and Applied Language Studies, Victoria University of Wellington (VUW), New Zealand

NAME (please print clearly):

Please read the following statements carefully.

- I agree to participate in this research project.

- I have been provided with enough information about the nature and objectives of this research.

- I have had an opportunity to ask questions and have had them answered to my satisfaction.

- I understand that a pseudonym will be used.

- I understand that any information I provide will be kept at a secure location, and will only be available to the researcher and her supervisors

- I understand that I can withdraw myself or any data I have provided by $30^{\text {th }}$ of December 2014.

- I understand that all research notes and data will be destroyed five years after the conclusion of the research

- I understand that the information I provide will be used only by Pakjira Leelertphan and her supervisors for this research project, publications and presentation arising from this research

Signed:

Date:

I would like to receive a summary of the results of this research when it is completed YES/NO

Email address to which a summary can be sent: 


\section{Appendix 5: Interview questions for hotel owners/web deigners}

1. How long have you been writing ?

2. What is your major responsibility in your organisation?

3. Who wrote/created your hotel website?

4. Who are your target group of customers?

5. What is the purpose of writing a hotel website ?

6. How did you decide on what to write on your websites in order to describe to customers about your hotels features including what your hotel has to offer?

7. Did you consult with other people or view other hotel websites to assist you with the writing content on your website?

8. Can you define boutique hotels ?

9. What makes your hotel a boutique hotel ?

10. What are the distinctive features of your hotel?

11. From my observation, there are several pronouns on your webpages, what did you try to convey through the use of pronoun you and we?

12. What did you try to convey through the use of adjectives and imperatives?

13. What kind of feedback do you expect to get from the readers?

14. How did you select color scheme, template/layout, images, position of the texts and images? 


\section{Appendix 6: Interview questions for web audiences}

1. Have you ever visited boutique hotels?

2. How many times?

3. Why do you choose to stay in boutique hotels? Did you choose the hotels yourself?

4. What are the most important features do you think boutique hotels should have?

5. What do you expect from staying at boutique hotels?

6. What is your overall satisfaction with your visit to boutique hotels?

7. What do you think about this hotel website? (Show the web readers the webpages in my datasets). 


\section{Appendix 7: An example of teaching materials}

\section{An example of Teaching materials}

The underlying value of the genre based approach is that it focuses on communicative purposes, in which genres are shaped by the context of the situation and the relationship between the writers and the audience as discussed in section 7.2.1. In addition, many genre scholars (Badger \& White, 2000; Bhatia, 1991; Flowerdew, 1993; Flowerdew, 2000; Peacock, 2002; Widodo, 2006) claim that the genre-based approach to discourse analysis contains various advantages for teaching teaching of English for Specific Purposes (ESP). These advantages are particularly salient in genre analyses that provide linguistic description and emphasise the social context where the genre is located. This section provides an example of the implementation of genre-based approach for the teaching of the organisational structures of boutique hotel webpages in a hotel and tourism class. This is an example of the kinds of teaching materials that I propose will contribute to the knowledge of non-native English students who would like to be able to write boutique hotel webpages in English. This proposed activity draws on the teaching models of various genre analysts (Badger \& White, 2000; Bhatia, 1991; Flowerdew, 1993; Flowerdew, 2000; Widodo, 2006). This activity is based on the move analysis of boutique hotel webpages in Thailand and New Zealand which represent the hotel practitioners' and web designers' communicative purposes. In this lesson, I mainly concentrate on one webpage (Suite page) that occurs in the Thai dataset as an example. However, the cross-cultural aspect of genre is important because its main focus is on an investigation of influence of cultures on similarities and differences across different texts within the same genre. This cross-cultural aspect could be introduced in later activities when the students are familiar with the concept of genre.

The fundamental objectives of boutique hotel webpages are to inform the prospective guests of the available services and facilities and persuade them to take actions. As a result, it is important to sensitise students to the structures and their conventionalities of this genre in order that they will be able to develop boutique hotel webpages. Although the generic structures of boutique hotel webpages are important aspect of genre that students have to acquire, it is important to inform the students that these structures are not rigid but flexible and may vary according to the contexts. I would like students to understand the characteristics of promotional texts that they will need to use to achieve the moves that occur in this Suite page. 


\section{Levels:}

This material is suitable for intermediate learners in a Tourism and Hotel major. I designed this teaching material to respond to their needs. Since many would like to go back to their hometown and open a small boutique hotel, in this course, these students would like to understand and practice how to develop hotel homepages. This material should be introduced after the students have been introduced to genre analysis and move structures. They should have a good understanding of what move structures are and how to identify moves in a variety of tourism and hotel texts. The following lesson displays the activities introducing them to the understanding of the characteristics of boutique hotel online promotional texts and how to demarcate moves within the texts.

Since the main communicative purposes of any genre is the most important element of a genre-based teaching approach (Peacock, 2002), in this activity, it is necessary to allow students to reflect on the overall objectives of boutique hotel online advertising texts and distinctive linguistic features of boutique hotels on webpages. The activity involves brainstorming, discussing and evaluating the possible objectives of this genre and typical linguistic features that are used to achieve the objectives of this genre. 


\section{Objectives and activities}

\begin{tabular}{|c|c|}
\hline Objectives & Activities \\
\hline $\begin{array}{l}\text { 1. Introduce students to boutique hotel } \\
\text { webpages }\end{array}$ & $\begin{array}{l}\text { 1. show students a list of salient } \\
\text { features/frequent content words emerging } \\
\text { from my datasets of boutique hotel webpages } \\
\text { in Thai and New Zealand (see Figure a) } \\
\text { 2. let students discuss in groups and guess what } \\
\text { kind of texts these typical linguistic features } \\
\text { come from }\end{array}$ \\
\hline $\begin{array}{l}\text { 2. Familiarise students with boutique hotel web } \\
\text { advertising texts }\end{array}$ & $\begin{array}{l}\text { 1. Provide students with a boutique hotel } \\
\text { online advertising text to scan and identify five } \\
\text { frequent words (distinctive linguistic features/ } \\
\text { frequent content words) and the overall } \\
\text { communicative purposes (see Figure b) } \\
\text { 2. Have students underline the words that they } \\
\text { think are distinctive linguistic features } \\
\text { representing boutiqueness based on the } \\
\text { frequency of occurrence } \\
\text { 3. Let students discuss in groups in order to } \\
\text { identify the main communicative purposes of } \\
\text { the texts by focusing on the whole idea of the } \\
\text { text }\end{array}$ \\
\hline $\begin{array}{l}\text { 3. Sensitise students to move structures in the } \\
\text { boutique hotel webpages }\end{array}$ & $\begin{array}{l}\text { 1. Ask students to think about the possible } \\
\text { move structures that can be used to achieve } \\
\text { the overall communicative purposes by looking } \\
\text { at the text at a sentence and paragraph level. } \\
\text { 2. Show students the move structure model of } \\
\text { the Suite (accommodation) page based on the } \\
\text { Thai dataset that I have identified from my } \\
\text { present study. Inform students that } \\
\text { awareness of variations within the same genre } \\
\text { is important. }\end{array}$ \\
\hline
\end{tabular}




\begin{tabular}{|c|c|}
\hline & $\begin{array}{l}\text { 3. Have students compare and contrast their } \\
\text { findings with my move structure model. After } \\
\text { that assess the variations. }\end{array}$ \\
\hline 4. Understand the move structure model & $\begin{array}{l}\text { 1. Ask students to use the move structure } \\
\text { model to identify the moves in the text } \\
\text { 2. Discuss the answers with the students and } \\
\text { ask them to discuss the function of all the } \\
\text { moves and how they are related to the texts. } \\
\text { 3. Provide students with new texts and ask } \\
\text { them to mark all the moves based on the same } \\
\text { model. }\end{array}$ \\
\hline $\begin{array}{l}\text { 5. Familiarise with typical linguistic features } \\
\text { that realise each move }\end{array}$ & $\begin{array}{l}\text { 1. Ask students to list distinctive linguistic } \\
\text { features of each move in the text } \\
\text { 2. Ask students to explain the relationship } \\
\text { between the forms of the linguistic features } \\
\text { and their functions } \\
\text { 3. Repeat the same process and exercises of the } \\
\text { same webpage (Suite page) with new texts } \\
\text { until they are confident. } \\
\text { 4. Discuss the findings with students and point } \\
\text { out that variation across the texts within the } \\
\text { same genre is common }\end{array}$ \\
\hline
\end{tabular}

The above activity encourages student to understand genre through authentic material within its situational context. This teaching material aims to offer students an explicit understanding of how texts are structured and why they are written the way they are. This activity also focuses on the importance of lexico-grammatical features that are used to achieve each move structure. For students, it is important to understand context of where a genre is situated and rhetorical structures that are used to fulfil communicative purposes of a genre. 


\section{Materials}

The following are the materials that are used to fulfil the above mentioned objectives and activities that I aim to propose in this section.

Figure a: Objective 1: a list of high frequency words

\begin{tabular}{|l|l|l|l|}
\hline room & deluxe & colonial & noble \\
\hline suites & balcony & pool & facilities \\
\hline
\end{tabular}

Figure b: Objective 2: a boutique hotel online advertising text

Charming, secluded and a homey - this is Puripunn Baby Grand Boutique Hotel. Take a step back in time and enjoy the traditional Lanna designs fused with colonial style. This is the perfect blend of aesthetics to create a refined and noble, yet comforting atmosphere. Small, with only 30 rooms, Puripunn uses this fact to its guests' advantage by providing personalized service to make each stay a memorable experience.

All rooms offer generous living areas, with high ceilings and wooden floors. A spacious bathroom with separate rain-shower room, toilet, and living area in all Puripunn Deluxe, Puripunn Family and suites. Each room has its own terrace/balcony with a tropical ceiling fan \& cozy bench overlooking the pool and a colonial-inspired easy chair wrapped in oriental-look fabric. The terrace is a favorite place for guests to sit back and relax all day long, making the guests feel as if they were in a noble residence of home away from home.

\section{In-room facilities and amenities}

- Private Balcony/Terrace with oversized bench and Colonial style easy chair in all rooms.

- Spacious bathroom with separate rain-shower room, toilet, and living area in all Puripunn Deluxe and Puripunn Family and suites.

- Loveseat.

- Air-conditioning with individual controls.

- Ceiling fan in all rooms.

- LEDTV

- DVD player in Puripunn Deluxe and all Suites

- Mini-stereo system in all suites

- IDD telephone

- Fully-stocked mini-bar

- In-room safe and hair-dryer in all room

- In-room complimentary tea-and coffee facilities

- Complimentary bottles of drinking water

- Desk and stationary

- Smoke detector

- Emergency torchlight 
Figure c: Objective 3: Move structure model for Suite (accommodation) page

\begin{tabular}{|l|}
\hline \multicolumn{1}{|c|}{ Moves } \\
\hline $\begin{array}{l}\text { Move I: Introducing the } \\
\text { hotel/facilities/services }\end{array}$ \\
\hline Move E: Establishing credentials \\
\hline Move DR- Describing the rooms \\
\hline
\end{tabular}

Figure d: Objective 4: The text with move structures identified

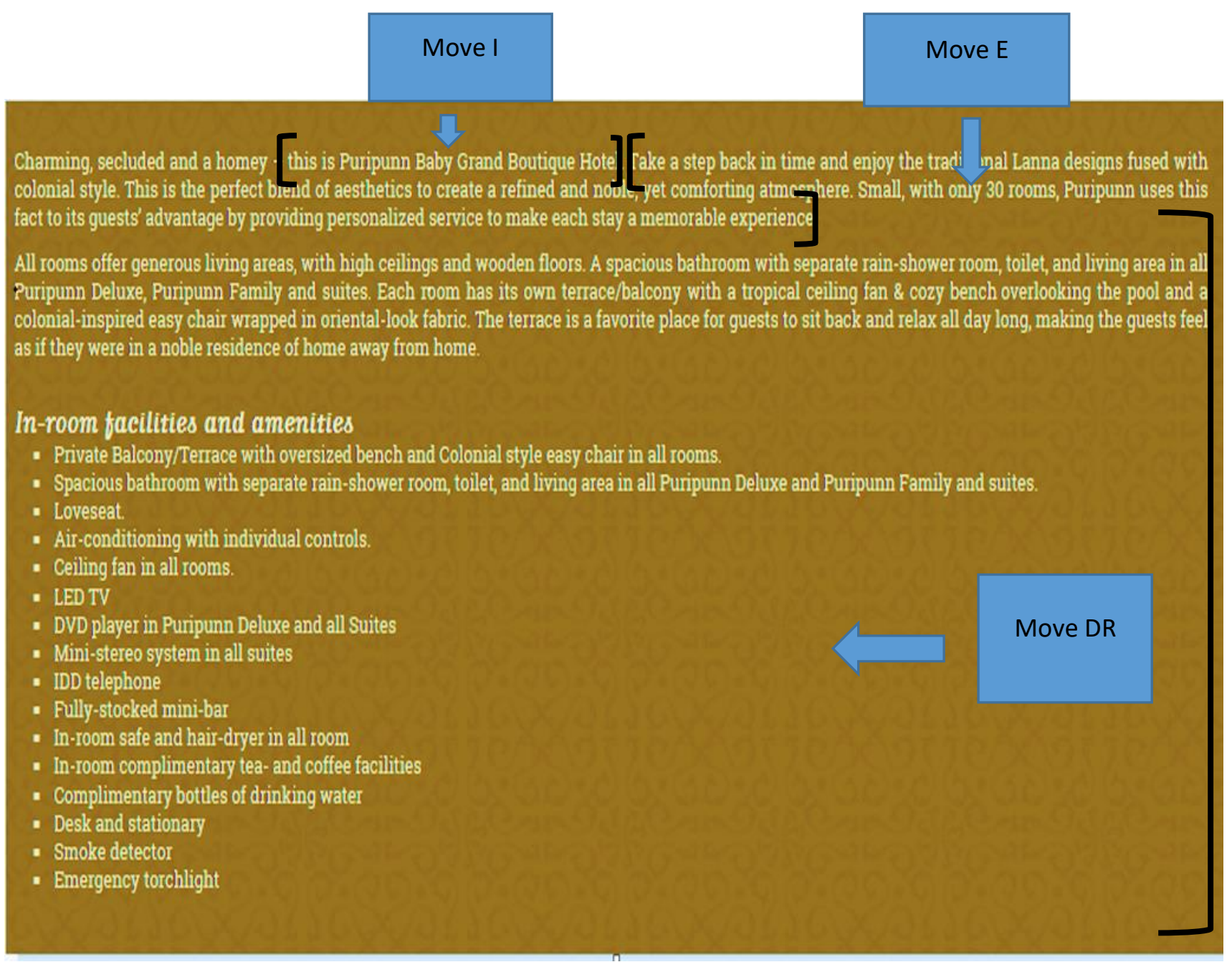

The above section presents a genre based teaching material, which is based on the move analysis of a Suite page of a Thai boutique hotel. The teaching material raises students' awareness of the importance of context and how genre is realised in a particular context. In addition, the activity focuses on how to identify move structures that are used to achieve the communicative purposes 
and linguistic choices that are associated with the genre. The students will be able to produce texts that conform to the rhetorical organisation. The next section discusses the profession implications. 
